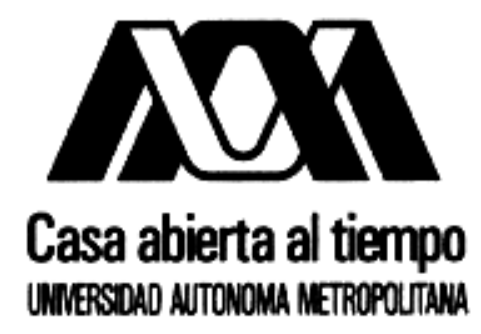

\title{
TRANSPORTE ONDULATORIO Y TIEMPOS DE RETARDO EN SISTEMAS MESOSCÓPICOS
}

Tesis que presenta:

\author{
Angel Marbel Martínez Argüello \\ Para obtener el grado de \\ Doctor en Ciencias (Física)
}

Asesor de Tesis:

Dr. Moisés Martínez Mares

Jurado:

Presidente: Dr. Héctor Manuel Moya Cessa

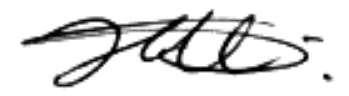

Secretario: Dr. Robin Preenja Sagar

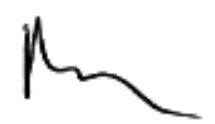

Vocal:

Dr. Thomas Gorin

Vocal: Dr. Julio César García Corte Julio Grar

Vocal: Dr. Moisés Martínez Mares

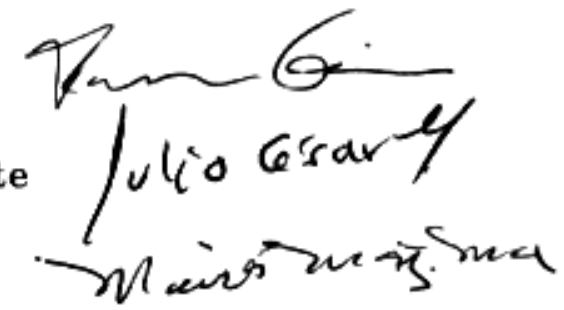




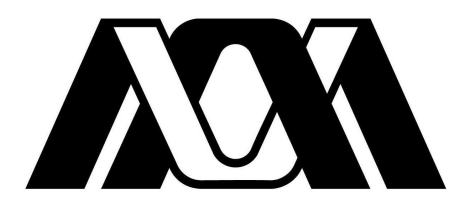

Casa abierta al tiempo

UNIVERSIDAD AUTONOMA METROPOLTANA

\title{
TRANSPORTE ONDULATORIO Y TIEMPOS \\ DE RETARDO EN SISTEMAS MESOSCÓPICOS
}

\author{
Tesis que presenta: \\ Angel Marbel Martínez Argüello \\ Para obtener el grado de \\ Doctor en Ciencias (Física)
}

Asesor de Tesis:

Dr. Moisés Martínez Mares

Jurado:

Presidente: Dr. Héctor Manuel Moya Cessa

Secretario: Dr. Robin Preenja Sagar

Vocal: $\quad$ Dr. Thomas Gorin

Vocal: Dr. Julio César García Corte

Vocal: $\quad$ Dr. Moisés Martínez Mares

Ciudad de México, 15 de julio de 2016 
Dedicado a:

mi familia, padres y hermanos, y mi Lizbeth por todo su apoyo, comprensión y cariño 


\section{Agradecimientos}

A mi familia, mis padres y mis hermanos, quienes me dieron la fortaleza y el apoyo para culminar esta etapa de mi vida. Un agradecimiento también muy especial a mi Lizbeth y Andelsito por ser una parte muy importante de mi vida y por ser mi alegría y motivación para seguir siempre adelante. No existen palabras para expresarles lo agradecido que les estoy.

Al Dr. Moisés Martínez Mares, a quien no sabría como pagar los años en los que me transmitió sus conocimientos, experiencia y entusiasmo, y por ser un excelente asesor. También, agradezco al grupo de ondas y materiales, al Dr. Rafael Méndez Sánchez y la Dra. Gabriela Báez; a los ahora Dres. Antonio Férnandez Marín, Victor Domínguez Rocha y Enrique Flores, y a los M. en C. Marcel Cobián Suárez, Filiberto Rámirez y Arturo Arreola; por sus valiosas contribuciones y las provechosas discusiones que tuvimos en nuestras múltiples reuniones de trabajo.

Agradezco a los Dres. Héctor Manuel Moya Cessa, Robin Preenja Sagar, Julio César García Corte y Thomas Gorin, por aceptar ser parte mi jurado y revisar minuciosamente el manuscrito y que con sus valiosas sugerencias me ayudaron a mejorar mi Tesis. Asimismo, agradezco a los Dres. José Antonio Méndez Bermúdez y Eleuterio Castaño Tostado quienes aceptaron amablemente formar parte del sínodo sin llegar a ser elegidos.

Sería inaceptable no incluir en estas líneas a mis amigos: Juan Carlos Ruelas, Ernesto Flores, Juan Carlos Sandoval, Víctor Ibarra, Julio César Hidalgo, Jairo Villafuerte y Samuel Pérez, quienes me permitieron robarles parte de su tiempo y que con su amistad me hicieron la vida más agradable.

Finalmente, quiero agradecer a la Universidad Autónoma Metropolitana Unidad Iztapalapa y al Departamento de Física por su hospitalidad y sobre todo por brindarme un espacio y un excelente ambiente de trabajo, así como al CONACyT por el apoyo económico brindado durante la realización de mis estudios de doctorado. 


\section{Índice general}

Resumen

$\begin{array}{ll}\text { Abstract } & \text { XIII }\end{array}$

1. Introducción 1

1.1. Propiedades de transporte en sistemas mesoscópicos cuánticos 1

1.2. Analogías clásico - cuánticas . . . . . . . . . . . . . . . 2

1.3. Fenómenos en procesos de dispersión . . . . . . . . . . . . . 3

1.4. Organización de la Tesis . . . . . . . . . . . . . . . . . 3

2. Dispersión, procesos directos y absorción 5

2.1. Dispersión de ondas y la matriz $S$. . . . . . . . . . . 5

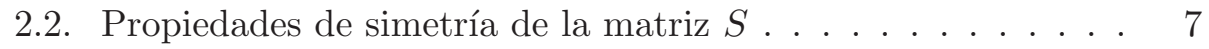

2.2.1. La conservación de flujo . . . . . . . . . . . . . . 7

2.2.2. Reversibilidad temporal . . . . . . . . . . . 7

2.3. Dispersión caótica . . . . . . . . . . . . . . . . 7

2.3.1. Ensambles circulares . . . . . . . . . . . . . 7

2.3.2. Formalismo de Heidelberg . . . . . . . . . . . . . . 8

2.4. Tiempos de retardo . . . . . . . . . . . . . . . . . . . 9

2.4.1. Tiempos de retardo propios . . . . . . . . . . . . . 10

2.4.2. Tiempos de retardo parciales . . . . . . . . . . . . 11

2.5. Procesos directos y absorción . . . . . . . . . . . . . . . . 12

2.5.1. Respuesta rápida . . . . . . . . . . . . . . . . 12

2.5.2. Acoplamiento imperfecto y procesos directos . . . . . 12

2.5.3. Absorción . . . . . . . . . . . . . . . . 13

2.5.4. Absorción y procesos directos . . . . . . . . . . . 14

3. Dispersión en una cavidad elástica unidimensional $\quad 17$

3.1. Propuesta experimental elástica . . . . . . . . . . . . . . . . . 17

3.1.1. Elemento dispersor con absorción . . . . . . . . . . 18 
3.1.2. Modelo elástico de la cavidad . . . . . . . . . . . . 20

3.2. Realización experimental . . . . . . . . . . . . . 23

3.2.1. El montaje experimental . . . . . . . . . . . 23

3.2.2. Resultado del experimento . . . . . . . . . . . 23

3.2.3. Un nuevo proceso directo . . . . . . . . . . . . . . . 25

3.3. Una nueva resonancia de Fano . . . . . . . . . . . . 26

3.3.1. Distribución de la resonancia . . . . . . . . . 28

4. Fluctuaciones universales del transporte electrónico 33

4.1. Transporte electrónico y dispersión . . . . . . . . . . . . . 33

4.2. Conductancia de un dispositivo electrónico de dos terminales 34

4.2.1. Fórmula de Landauer . . . . . . . . . . . . . . . 34

4.2.2. Fluctuaciones en un punto cuántico caótico . . . . . . 35

4.2.3. Analogía elástica . . . . . . . . . . . . . . 36

4.3. Caída de voltaje en un alambre cuántico . . . . . . . . . 37

4.3.1. Dispositivo electrónico de tres terminales . . . . . . 38

4.3.2. Voltaje en un conductor ideal . . . . . . . . . . . 39

4.3.3. Fluctuaciones del voltaje en un alambre desordenado . 40

4.3.4. Fluctuaciones del voltaje en un alambre caótico . . . . 41

4.4. Bombeo de corriente en un punto cuántico . . . . . . . . . . . 46

4.4.1. Bombeo cuántico paramétrico . . . . . . . . . 46

4.4.2. Fluctuaciones de la corriente bombeada . . . . . . . 48

4.4.3. Análisis de matrices aleatorias para la corriente . . . . 48

5. Tiempos de retardo $\quad \mathbf{5 1}$

5.1. Momentos generalizados de los tiempos de retardo propios . . 51

5.1.1. Momentos negativos de la distribución de Laguerre de

$N$ variables .................. 53

5.1.2. $\beta$ número entero par . . . . . . . . . . . . 55

5.2. Cálculos explícitos para $\beta=1 \ldots \ldots \ldots$. . . . . 56

5.2.1. Caso $N=1 \ldots \ldots \ldots \ldots \ldots$. . . . . . . . 57

5.2.2. Caso $N=2 \ldots \ldots \ldots \ldots \ldots \ldots$

5.3. Cálculos explícitos para $\beta=2 \ldots \ldots \ldots . \ldots 58$

5.3.1. Caso $N=1 \ldots \ldots \ldots \ldots \ldots$

5.3.2. Caso $N=2 \ldots \ldots \ldots \ldots \ldots$

5.3.3. Caso $N=3 \ldots \ldots \ldots \ldots \ldots$

5.3.4. Caso $N=4 \ldots \ldots \ldots \ldots \ldots$. . . . . . . . 61

5.4. Cálculos explícitos para $\beta=4 \ldots \ldots \ldots$. . . . . 63

5.4.1. Caso $N=1 \ldots \ldots \ldots \ldots \ldots$

5.4.2. Caso $N=2 \ldots \ldots \ldots \ldots \ldots$ 
5.5. Expresiones generales para los momentos conjuntos . . . . . . 64

5.6. Constante de normalización de la distribución de Laguerre . . 68

5.7. Tiempos de retardo parciales . . . . . . . . . . . 70

5.7.1. Simulaciones numéricas con matrices aleatorias . . . . 71

$\begin{array}{ll}\text { 6. Conclusiones y perspectivas } & 73\end{array}$

A. Conceptos básicos de RMT $\quad 77$

A.1. Ensembles Gaussianos . . . . . . . . . . . . . . . 77

A.1.1. Ensemble ortogonal Gaussiano . . . . . . . . . . 78

A.1.2. Ensemble unitario Gaussiano . . . . . . . . . . 78

A.1.3. Ensemble simpléctico Gaussiano . . . . . . . . 79

A.2. Ensembles circulares . . . . . . . . . . . . . . 79

B. Distribuciones marginales $\quad 81$

B.1. Distribución marginal radial . . . . . . . . . . . . . . . . . 82

B.2. Distribución marginal angular . . . . . . . . . . . . . 83

C. Fluctuaciones del voltaje $\quad \mathbf{8 5}$

C.1. Matriz $S$ de un dispositivo de tres terminales . . . . . . . 85

C.1.1. Conductor 1 caótico y conductor 2 perfecto con simetría $\beta=1 \ldots \ldots \ldots . \ldots . \ldots 8$

C.1.2. Conductor 1 caótico y conductor 2 perfecto con simetría $\beta=2 \ldots \ldots \ldots . \ldots . \ldots . \ldots$

D. Propiedades asociadas a $F_{\theta_{n}} \quad 91$ 


\section{Resumen}

En la actualidad, el desarrollo de nuevas técnicas experimentales de medición ha permitido la fabricación controlada de estructuras artificiales de tamaño micrométrico y nanométrico. En esos sistemas la coherencia de fase de las ondas determina algunas características importantes de su comportamiento. Muchos de esos sistemas pertenecen al dominio de la física mesoscópica.

En esta Tesis estudiamos algunos de los rasgos más importantes del transporte coherente. Primero, estudiamos el transporte de ondas en una cavidad elástica unidimensional, en presencia de absorción y de reflexiones directas que dan origen a procesos directos, desde el punto de vista tanto teórico como experimental. Este sistema corresponde con el de una cavidad unidimensional cuántica en presencia de absorción y de procesos directos. Después, estudiamos el transporte electrónico en una cavidad bidimensional con dos puertos, en donde omitimos las pérdidas y los procesos directos, pero agregamos la complejidad que implica el caos. Observamos que debido al caos la transmisión de ondas muestra fluctuaciones que son de carácter universal; es decir, que sólo dependen de las simetrías presentes en el sistema mismo. Estas fluctuaciones las analizamos de forma experimental en el correspondiente sistema análogo, en una cavidad elástica. Observamos que aunque el sistema elástico presenta disipación de energía, esta no rompe la coherencia de fase de las ondas. También estudiamos las fluctuaciones de la caída de voltaje en un dispositivo electrónico de tres terminales y las de la corriente bombeada en un punto cuántico, así como el efecto que las simetrías tienen sobre ellas. Encontramos que las fluctuaciones de la corriente bombeada dependen de los momentos generalizados de los tiempos propios de retardo de Wigner-Smith. En la última parte de la Tesis nos enfocamos en el cálculo de estos momentos para toda clase de simetría, así como para cualquier número de canales. 


\section{Abstract}

Nowadays, the development of new experimental techniques of measurement have allowed the controlled fabrication of artificial structures of size of micrometers and nanometers. In those systems, the wave phase coherence determines some important characteristics of their behavior. Many of those systems belong to the domain of mesoscopic physics.

In this Thesis we study some of the most important features of coherent transport. First, we study the wave transport in a one-dimensional elastic cavity, in presence of both absorption and direct reflexions that give rise to direct processes. This system corresponds to a one-dimensional quantum cavity in presence of absorption and direct processes. Then, we study the electronic transport through two-dimensional cavity with two ports in which we avoid the power losses and direct processes, but we add the complexity that implies chaos. We observe that due to chaos the transmission of waves shows fluctuations that are of universal character; that is to say, they only depend on the symmetries present in the system itself. We analyze these fluctuations in an experimental way in its corresponding analogous system, an elastic cavity. Although the elastic system has losses of energy, we observe that the ondulatory phase coherence is not broken. We also study the fluctuations of the voltage drop in a three-terminal electronic device and in the pumped current through a quantum dot; as well as the effect that symmetries have on them. We find that the fluctuations of the pumped current depend on the negative moments of the Wigner-Smith proper delay times. In the last part of this Thesis we focus on the calculation of these moments, and we present their generalization for all symmetry classes as well as for any number of channels. 


\section{Capítulo 1}

\section{Introducción}

\subsection{Propiedades de transporte en sistemas me- soscópicos cuánticos}

El estudio de las propiedades de dispersión y de transporte en los llamados sistemas mesoscópicos ha sido motivo de extensos trabajos de investigación en las últimas décadas, tanto teóricos como experimentales. Estos sistemas abarcan no sólo a la física cuántica sino también a otras ramas de la física tales como la acústica, la elasticidad, la óptica y el electromagnetismo.

Por sistema mesoscópico se entiende un sistema de transporte ondulatorio coherente, el cual puede ser tanto clásico como cuántico [1]. En estos sistemas la fase de la correspondiente función de onda no se pierde durante su viaje a través del sistema $[1,2]$. Esto se logra haciendo que las dimensiones del sistema sean más pequeñas que el camino libre medio inelástico. Así, por ejemplo, en la actualidad el desarrollo de nuevas técnicas experimentales de medición ha permitido la fabricación de dispositivos artificiales electrónicos y optoelectrónicos, cuyas medidas van desde micras hasta nanómetros, donde el transporte electrónico se establece en forma coherente [3, 4] y están bien descrito por la mecánica cuántica $[1,5,6,7,8]$. Estos dispositivos se pueden construir sobre un gas bidimensional de electrones libres, formado en la interfaz entre dos medios semiconductores, los más comunes son de Arsenuro de Galio (GaAs) y Arsenuro de Galio Aluminio (AlGaAs). Sobre esta interfaz se colocan contactos metálicos puestos a potenciales electrostáticos fijos que al repeler a los electrones que están justo debajo (algunos nanómetros) dan la forma de la cavidad. A estos sistemas también se les conoce como puntos cuánticos, o sistemas de cero dimensiones. En estos, los electrones entran al punto cuántico desde los reservorios electrónicos, también a 
través de contactos metálicos. Una vez dentro del sistema, los electrones sólo pueden chocar con las paredes que los confinan $[2,7]$. Entre las principales cantidades que se estudian en el transporte electrónico están la conductancia, sus derivadas energética y paramétrica, la caída de voltaje y la corriente bombeada en ausencia de un voltaje aplicado [9, 10]. Asimismo, el tiempo de retardo de Wigner es de interés científico pues da una medida del tiempo de interacción de la partícula cuántica con el sistema y determina el comportamiento de la capacitancia mesoscópica [11], la termopotencia [12]; las derivadas paramétricas [9] y la corriente bombeada, entre otras [12], están determinadas por los tiempos propios de retardo.

\subsection{Analogías clásico - cuánticas}

El estudio de las propiedades de transporte en sistemas coherentes generalmente se puede reducir a un problema de dispersión [2]. La ecuación de onda hace que, en particular, los sistemas ondulatorios clásicos, aparte de su importancia intrínseca, sirvan como modelos análogos de los sistemas cuánticos. En ambos casos la dinámica está descrita por la ecuación de onda, que en su forma estacionaria se reduce a la ecuación de Helmholtz.

Como ejemplos de sistemas ondulatorios clásicos encontramos las cavidades y gráficas de microondas $[13,14,15]$, los sistemas elásticos [16] y acústicos [17, 18], y las microcavidades ópticas [19]. En ellos las cantidades relevantes son la matriz de dispersión, el coeficiente de transmisión como análogo de la conductancia, y su derivada con respecto a la frecuencia como análogo de la derivada energética de la conductancia en un sistema cuántico [20].

En las últimas décadas se ha estudiado el transporte en cavidades cuya dinámica en el límite de rayos es caótica ${ }^{1}$. Esto se debe a que es difícil controlar la forma de un sistema cuántico a nivel microscópico. En ese sentido las cavidades clásicas tiene la ventaja de que son más fáciles de construir, pero se eligen a propósito caóticas para que sirvan como análogos de los sistemas cuánticos. En estos casos, las cantidades de transporte, o de dispersión en general, fluctúan con respecto de parámetros externos como son: un campo magnético externo aplicado, la energía de Fermi de los electrones incidentes y el parámetro que da la geometría del punto cuántico [21, 22, 23]; la frecuencia y el perfil, en las cavidades ondulatorias clásicas [24]. Entonces, su descripción se da en términos de un análisis estadístico.

\footnotetext{
${ }^{1}$ Asimismo, se ha estudiado el transporte en sistemas cuánticos con impurezas, llamados desordenados, que hace que el transporte sea difusivo.
} 
Desde el punto de vista teórico se ha mostrado que para sistemas caóticos, la teoría de matrices aleatorias da una descripción correcta de las fluctuaciones estadísticas en las propiedades de transporte y de dispersión [1, $7,8,25]$. En este análisis se pierden detalles del problema, pero se resaltan los aspectos universales del fenómeno, como son las simetrías asociadas al sistema. Ejemplos de ello, son la presencia o ausencia de invariancia ante inversión temporal [25] y la simetría espacial de reflexión que también tiene efectos importantes en el transporte [26].

\subsection{Fenómenos en procesos de dispersión}

No obstante la existencia de esta analogía es importante mencionar que, desde el punto de vista experimental, los sistemas ondulatorios clásicos muestran algunas características interesantes con respecto a los cuánticos. Por ejemplo, existe disipación de energía debido al fenómeno de absorción, por lo que el flujo no se conserva. Una de las cuestiones de mayor interés consiste en modelar estas pérdidas de potencia [27, 28]. Además, una situación a considerar es que en los experimentos las antenas que alimentan al sistema nunca acoplan de manera perfecta, lo cual da lugar a una respuesta rápida debido a las reflexiones directas. Es bien sabido que estos procesos directos tienen un efecto drástico en el transporte, tanto en sistemas clásicos como cuánticos

Un primer análisis del efecto de la absorción y los procesos directos, se realizó con un modelo unidimensional en mecánica cuántica, que consiste de un potencial tipo delta de Dirac, situado a una cierta distancia de una pared impenetrable, pero con intensidad compleja [29]. Una propuesta más realista que parte de la elasticidad se hace en la presente Tesis, como veremos más adelante. Además, en una realización experimental encontramos un nuevo proceso directo debido a procesos de medición.

\subsection{Organización de la Tesis}

En la búsqueda de nuestros objetivos, abordaremos los diferentes procesos que ocurren en los sistemas ondulatorios uno a la vez. Así, en el próximo capítulo presentamos el fenómeno de dispersión de ondas, el de procesos directos y el de absorción. También, definimos el concepto de matriz de dispersión y damos sus principales propiedades de simetría, así como el formalismo matemático que usaremos para el estudio de sus principales propiedades. 
En el capítulo 3 analizaremos un sistema sencillo unidimensional que no involucra la complejidad del caos pero que, sin embargo, contiene tanto absorción como procesos directos que afectan drásticamente la dispersión de ondas. Este estudio lo realizaremos desde el punto de vista tanto teórico como experimental.

En el capítulo 4 abordaremos el problema de la dispersión de ondas y el transporte en sistemas bidimensionales. Por la complejidad del caos, nos concentraremos en el transporte ondulatorio sin absorción ni procesos directos. Analizaremos las fluctuaciones de la transmisión de ondas a través de un punto cuántico caótico, así como su analogía en un sistema elástico. También, estudiaremos las fluctuaciones en un dispositivo electrónico de tres terminales, y las fluctuaciones de la corriente bombeada en un punto cuántico así como el efecto que las simetrías presentes tienen sobre él. Veremos que estas dependen de los momentos generalizados de los tiempos de retardo propios. Por tanto, en el capítulo 5 determinaremos estos momentos para un número arbitrario de canales así como de cualquier simetría, cuando el acoplamiento entre las guías de onda que alimentan la cavidad es ideal. En el capítulo 6 presentamos nuestras conclusiones así como las perspectivas de esta Tesis.

Finalmente, algunos conceptos sobre la teoría de matrices aleatorias que usamos en la Tesis, así como detalles del cálculo de algunos resultados que presentamos en el texto, los incluimos en los apéndices correspondientes. 


\section{Capítulo 2}

\section{Dispersión, procesos directos y absorción}

En este capítulo presentamos un resumen sobre uno de los conceptos más usados en el estudio de los fenómenos ondulatorios y su relación con fenómenos de transporte: el de la matriz de dispersión $S$ y sus principales propiedades de simetría bajo la condición de conservación de flujo. Dado que la propiedad física que nos interesa estudiar es el tiempo de retardo de Wigner introducimos este concepto en este capítulo. También, revisamos los conceptos de matriz $S$ óptica que representa los procesos directos en el sistema y dos modelos que describen la absorción en sistemas en los que no se cumple la conservación del flujo. Planteamos un camino para estudiar el efecto tanto de procesos directos como de absorción en el tiempo de retardo.

\subsection{Dispersión de ondas y la matriz $S$}

Mucho del conocimiento que tenemos acerca de la estructura de la materia a nivel microscópico se extrae de experimentos de dispersión de ondas o de partículas. En estos experimentos se envía un haz o flujo de partículas hacia el blanco en específico que se desea estudiar, y por medio de detectores se cuenta el número de partículas dispersadas en un cierto ángulo, el cual es proporcional al flujo incidente y a la sección eficaz de dispersión. En mecánica cuántica en la aproximación de partícula independiente, un electrón se representa por un paquete de ondas, que es una superposición de ondas planas. Cuando una onda incide sobre un blanco, ésta se dispersa en general con una amplitud y dirección diferente al de la onda incidente, la cual se conoce como amplitud de dispersión. 


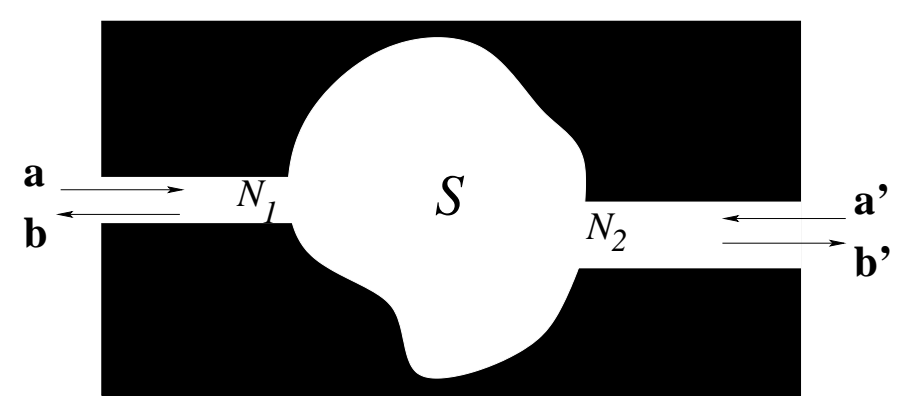

Figura 2.1: Cavidad dispersiva conectada de manera ideal a dos reservorios electrónicos por medio de guías de onda electrónicas con $N_{1}$ y $N_{2}$ modos de propagación, respectivamente.

En un problema de dispersión estacionario, o independiente del tiempo, las amplitudes de las ondas planas dispersadas se pueden expresar en términos de las amplitudes de las ondas planas incidentes por medio de una cantidad que las relaciona conocida como matriz de dispersión, $S$, la cual contiene toda la información del blanco dispersor [1].

Consideremos una región de dispersión, en particular consideremos una cavidad conectada de manera perfecta a dos reservorios electrónicos por medio de guías de onda, cada una de las cuales soporta $N_{1}$ y $N_{2}$ modos de propagación, respectivamente (ver la figura 2.1). En el lenguaje de la física nuclear a estos modos de propagación se les conoce como canales. La matriz de dispersión relaciona las amplitudes de las ondas salientes de la cavidad en los distintos canales con las de las ondas entrantes. Si las amplitudes de las ondas entrantes se escriben en forma de matriz columna como a $=\left(a_{1}, \ldots, a_{N}\right)^{T}$ para las ondas incidentes desde el lado izquierdo (el superíndice $T$ indica la operación transpuesta del vector), como $\mathbf{a}^{\prime}=\left(a_{1}^{\prime}, \ldots, a_{N}^{\prime}\right)^{T}$ para las incidentes desde el lado derecho, y como $\mathbf{b}=\left(b_{1}, \ldots, b_{N}\right)^{T}$ y $\mathbf{b}^{\prime}=\left(b_{1}^{\prime}, \ldots, b_{N}^{\prime}\right)^{T}$ para las amplitudes de las ondas salientes desde el lado izquierdo y derecho, respectivamente; por definición

$$
\left(\begin{array}{c}
\mathbf{b} \\
\mathbf{b}^{\prime}
\end{array}\right)=S\left(\begin{array}{c}
\mathbf{a} \\
\mathbf{a}^{\prime}
\end{array}\right)
$$

La matriz $S$ es una matriz de dimensión $N \times N$ donde $N=N_{1}+N_{2}$ es el número total de canales. 


\subsection{Propiedades de simetría de la matriz $S$}

\subsubsection{La conservación de flujo}

En la sección anterior vimos que cualquier problema de dispersión se puede describir por medio de la matriz $S$. En ausencia de cualquier tipo de simetría en el sistema, la única restricción que se debe satisfacer es la condición de conservación del flujo, lo que implica que la matriz $S$ debe ser una matriz unitaria; es decir

$$
S S^{\dagger}=S^{\dagger} S=\mathbf{1}_{N},
$$

donde el superíndice $†$ significa la operación transpuesta y conjugada de la matriz, y $\mathbf{1}_{N}$ es la matriz identidad de $N \times N$. La condición (2.2) es una consecuencia de la ecuación de continuidad que expresa la conservación local del flujo de probabilidad.

\subsubsection{Reversibilidad temporal}

Si además de satisfacer la condición de conservación de flujo, ecuación (2.2), el problema tiene simetría ante inversión en el tiempo (TRI por sus siglas en inglés) y espín entero, o espín semi-entero y simetría de rotación, la matriz $S$ es una matriz simétrica; es decir [1]

$$
S=S^{T},
$$

donde el superíndice $T$ denota la transpuesta de la matriz.

\subsection{Dispersión caótica}

\subsubsection{Ensambles circulares}

Cuando la región de dispersión tiene una forma que induce una dinámica caótica en el límite de la óptica de rayos, las propiedades de dispersión fluctúan como función de parámetros externos como pueden ser un campo magnético o la energía de Fermi en sistemas electrónicos, o parámetros que modifican la forma de los sistemas. En esos casos puede ser conveniente un análisis estadístico de dichas propiedades. Aunque en este análisis se pierde mucha de la información microscópica de los sistemas, se resaltan sus propiedades universales.

En el caso de cavidades caóticas se ha mostrado que la teoría de matrices aleatorias [30] (RMT por sus siglas en inglés) da una descripción apropiada de sus propiedades estadísticas. En esta teoría un ensemble, o conjunto 
representativo de cavidades, se describe a través de un ensemble de matrices $S$ distribuidas uniformemente en el espacio de matrices $S$, sujetas a las condiciones de simetría presentes en el problema de dispersión ${ }^{1}$. Entonces, la matriz $S$ pertenece a uno de los tres ensembles circulares de RMT, conocidos como COE, CSE y CUE (ensembles circulares ortogonal, simpléctico y unitario, respectivamente, por sus siglas en inglés). El ensemble COE se obtiene cuando el sistema satisface la simetría TRI, espín entero o espín semientero con simetría de rotación. En presencia de simetría de TRI, espín semientero y ausencia de simetría de rotación, la matriz $S$ es self-dual y el ensemble al que pertenece es el CSE. En ausencia de cualquier simetría, la única restricción es la conservación de flujo y el ensemble correspondiente es el CUE. En el esquema de Dyson estos ensembles están etiquetados como $\beta=1,4$ y 2 , respectivamente ${ }^{2}[2,30,31]$. Entonces, en estos ensembles la densidad de probabilidad de la matriz $S$ es constante

$$
p_{0}^{(\beta)}(S)=1
$$

En consecuencia, se satisface que el promedio sobre el ensemble de la matriz $S,\langle S\rangle$, también conocida como matriz $S$ óptica, es nulo: $\langle S\rangle=0^{3}[1]$.

En este formalismo se hace la estadística directamente sobre la matriz $S$ definida a una sola energía de Fermi, $\mathcal{E}$. Por tanto, al no mostrar la dependencia explícita con $\mathcal{E}$ no es posible determinar el comportamiento de cantidades que dependan de la derivada con respecto a este parámetro. Sin embargo, existe una conjetura debida a Wigner que hace posible el cálculo indirecto de esta derivada $[11,12]$. Además, existe otra alternativa que nos permite determinar la derivada de la matriz $S$ con respecto a la energía, conocida como formalismo de Heidelberg. En la siguiente sección describimos este formalismo.

\subsubsection{Formalismo de Heidelberg}

En el formalismo de Heidelberg, también conocido como formalismo energético, la matriz $S$ se escribe explícitamente en términos de la energía $\mathcal{E}$ y del Hamiltoniano $H$ que describe sólo a la cavidad. En este modelo se asume que aunque el número de modos internos de la cavidad es muy grande,

\footnotetext{
${ }^{1}$ Esto representa la idea del postulado de iguales probabilidades a priori [1].

${ }^{2}$ En el apéndice A presentamos un resumen de algunos conceptos básicos de RMT que usaremos en esta Tesis.

${ }^{3}$ El subíndice 0 en la ecuación (2.4) se refiere al promedio de $S$ nulo.
} 
$H$ es de dimensión finita $M$. La matriz $S$ está dada por [32]

$$
S(\mathcal{E})=\mathbf{1}_{N}-2 \mathrm{i} \pi W^{\dagger} \frac{1}{\mathcal{E} \mathbf{1}_{M}-H+\mathrm{i} W W^{\dagger}} W,
$$

donde $W$ es una matriz de $M \times N$, independiente de la energía, que acopla los $N$ modos de propagación en las guías de onda con los $M$ estados internos de la cavidad. Para acoplamiento ideal de canales equivalentes descorrelacionados los elementos de la matriz de acoplamiento $W, W_{m n}$, son

$$
W_{m n}=\frac{\sqrt{M \Delta}}{\pi} \delta_{m n}, \quad \text { con } \quad m=1, \ldots, M, \quad \text { y } \quad n=1, \ldots, N,
$$

y $\delta_{m n}$ es la delta de Kronecker.

Para sistemas caóticos, el Hamiltoniano $H$ que describe al sistema es una matriz aleatoria que pertenece a uno de los tres ensembles Gaussianos, los cuales se denotan como GOE, GSE y GUE (ensemble Gaussiano ortogonal, simpléctico y unitario por sus siglas en inglés, respectivamente), dependiendo de la simetría presente en el sistema. Los elementos de matriz de $H$ son variables aleatorias descorrelacionadas distribuidas de acuerdo con una ley de probabilidad Gaussiana con media cero y varianza $\lambda^{2} \beta / M$, la cual determina el espaciamiento medio de niveles en el centro de la banda $\Delta=\pi \lambda / M$.

Este formalismo es más adecuado si se quiere conocer la dependencia de las cantidades de dispersión con la energía. Sin embargo, la estadística se hace a nivel del Hamiltoniano tomado de los ensembles Gaussianos para luego construir la estadística de la matriz $S$, en lugar de hacer la estadística directamente sobre la matriz $S$ como en el caso de los ensembles circulares.

\subsection{Tiempos de retardo}

El tiempo de retardo fue introducido primero por Wigner para el caso de un canal y representa el retraso sufrido por un paquete de ondas debido a su interacción con un potencial dispersor [33]. En el caso de muchos canales, este concepto fue generalizado por Smith a través de lo que se conoce como la matriz de tiempos de retardo de Wigner-Smith, $Q_{W}$ [34]. Esta matriz es una matriz Hermitiana de dimensión $N$ que está dada en términos de la matriz de dispersión $S$ y de su derivada con respecto a la energía. En unidades del tiempo de Heisenberg, $\tau_{H}=2 \pi \hbar / \Delta, Q_{W}$ está dada por

$$
Q_{W}=-\mathrm{i} \frac{\hbar}{\tau_{H}} S^{\dagger} \frac{\partial S}{\partial \mathcal{E}} .
$$


Esta matriz es una cantidad importante para la descripción de las propiedades de transporte que dependan de la matriz $S$ y de su derivada con respecto a la energía.

Los valores propios de $Q_{W}$ se conocen como tiempos de retardo propios y representan los tiempos de retardo en cada uno de los canales. Estos juegan un papel muy importante en la descripción de las fluctuaciones estadísticas de varias propiedades de transporte en cavidades clásicamente caóticas, como son los puntos cuánticos y las cavidades de microondas con acoplamiento ideal, entre otras [12]. En particular, el tiempo de retardo de Wigner queda definido como el promedio de los tiempos de retardo propios.

\subsubsection{Tiempos de retardo propios}

Una manera conveniente de escribir la matriz de tiempos de retardo es la forma simetrizada de la matriz de Wigner-Smith, que en unidades adimensionales es [35]

$$
Q=-\frac{\mathrm{i} \hbar}{\tau_{H}} S^{-1 / 2} \frac{\partial S}{\partial \mathcal{E}} S^{-1 / 2}
$$

La matriz $Q$ es una matriz Hermitiana para $\beta=2$, es simétrica real para $\beta=1$ y autodual cuaterniónica para $\beta=4$. Los valores propios de $Q$, $q_{i}$ 's $(i=1, \ldots, N)$, son los tiempos de retardo propios medidos en unidades de $\tau_{H}$; es decir, $q_{i}=\tau_{i} / \tau_{H}$. La distribución conjunta de estos tiempos se conoce en términos de sus recíprocos, $x_{i}=\tau_{H} / \tau_{i}$, y está dada por el ensemble de Laguerre [35]

$$
P_{\beta}\left(x_{1}, \ldots, x_{N}\right)=C_{N}^{(\beta)} \prod_{i<j}^{N}\left|x_{i}-x_{j}\right|^{\beta} \prod_{k=1}^{N} x_{k}^{\beta N / 2} \mathrm{e}^{-\beta x_{k} / 2},
$$

donde $C_{N}^{(\beta)}$ es una constante de normalización, definida a través de la condición

$$
\int_{0}^{\infty} P_{\beta}\left(x_{1}, \ldots, x_{N}\right) d x_{1} \cdots d x_{N}=1 .
$$

Una característica que presenta la distribución de Laguerre es la repulsión entre los tiempos propios, la cual es heredada de la repulsión de niveles entre los valores propios del Hamiltoniano que describe la cavidad cerrada [35]. Aunque la distribución de Laguerre ha sido ampliamente usada, la constante $C_{N}^{(\beta)}$ no se conoce debido a la dificultad matemática que representa 
la repulsión en los tiempos. En la presente Tesis determinamos esta constante de normalización a través de un método inductivo que presentamos en el capítulo 5. En ese mismo capítulo presentamos el cálculo de momentos conjuntos de los tiempos propios que se requieren en la determinación de otras cantidades de transporte.

\subsubsection{Tiempos de retardo parciales}

Otras cantidades útiles en la caracterización de la dispersión caótica son los tiempos de retardo parciales que se definen como la derivada con respecto a la energía de las fases propias, o eigenfases, de la matriz $S$. En forma diagonal, ésta se puede escribir como

$$
S=U E U^{\dagger}
$$

donde $U$ es una matriz unitaria de dimensión $N$ que se forma con los vectores propios de $S$ y $E$ es una matriz diagonal; es decir,

$$
E_{i j}=\mathrm{e}^{\mathrm{i} \theta_{i}} \delta_{i j},
$$

con $\delta_{i j}$ la delta de Kronecker. La matriz de tiempos de retardo parciales $\hat{\tau}$ es una matriz diagonal de $N \times N$ que se define en términos de la derivada con respecto a la energía de la forma diagonal de la matriz $S$; en unidades adimensionales es [36, 37]

$$
\hat{\tau}=-\frac{\mathrm{i} \hbar}{\tau_{H}} E^{-1} \frac{\partial E}{\partial \mathcal{E}}
$$

y sus elementos

$$
\tau_{s}=\frac{\hbar}{\tau_{H}} \frac{\partial \theta_{s}}{\partial \mathcal{E}}
$$

son los tiempos de retardo parciales. Aunque estos tiempos están correlacionados, sus correlación son de una naturaleza diferente a las correlaciones que existen entre los tiempos de retardo propios, ya que no muestra la repulsión de niveles [38].

En el caso de un solo canal, los tiempos de retardo propios y los tiempos de retardo parciales concuerdan con el tiempo de retardo de Wigner cuya distribución se conoce para toda clase de simetría $\beta=1,2$ y $4[11,36,39]$. Para el caso general de un número arbitrario de canales se conoce la distribución de un tiempo parcial para cualquier $\beta$, a excepción de una constante de normalización [38]. Este resultado generaliza un resultado previo para 
$\beta=2$ con una normalización apropiada [36, 40]. También se conocen resultados numéricos para $\beta=1$ y 2 , aunque sólo el caso $\beta=2$ concuerda con las predicciones teóricas [37]; el caso $\beta=4$ rara vez se discute. En esta Tesis presentamos resultados teóricos en donde calculamos la constante de normalización adecuada, así como resultados para el caso $\beta=4$ que verificamos con simulaciones basadas en RMT (ver el capítulo 5).

\subsection{Procesos directos y absorción}

\subsubsection{Respuesta rápida}

Hasta aquí hemos considerado el caso en el cual el acoplamiento entre las guías de onda y la cavidad es ideal. Sin embargo, en una situación realista el acoplamiento no es perfecto y esto produce reflexiones de las ondas antes de ingresar a la cavidad, que se conocen como procesos directos y que representan la respuesta rápida del sistema, las cuales deben tomarse en cuenta en el proceso de dispersión.

En presencia de procesos directos la matriz $S$ ya no se distribuye uniformemente, de manera que la matriz $S$ óptica ya no es nula, $\langle S\rangle \neq 0$; de hecho $\langle S\rangle$, el promedio sobre un ensemble de la matriz $S$, cuantifica dichos procesos directos. En ese caso, la densidad de probabilidad de $S$ está dada por lo que se conoce como el kernel de Poisson,

$$
p_{\langle S\rangle}^{(\beta)}(S)=\frac{\left[\operatorname{det}\left(\mathbf{1}_{N}-\langle S\rangle\langle S\rangle^{\dagger}\right)\right]^{(\beta N+2-\beta) / 2}}{\left|\operatorname{det}\left(\mathbf{1}_{N}-S\langle S\rangle^{\dagger}\right)\right|^{\beta N+2-\beta}} .
$$

Notemos que cuando $\langle S\rangle=0$ el kernel de Poisson se reduce a la distribución uniforme (constante) de la ecuación (2.4).

\subsubsection{Acoplamiento imperfecto y procesos directos}

Físicamente, las reflexiones directas se pueden modelar por medio de una barrera de tunelaje entre las guías de onda y la cavidad. Uno puede imaginar que todas las guías están juntas en una superguía y que una matriz de dispersión $S_{b}$ de la barrera acopla esta superguía con la cavidad. Esta matriz $S_{b}$, es un matriz unitaria de dimensión $2 N$ que tiene la estructura

$$
S_{b}=\left(\begin{array}{cc}
r_{b} & t_{b}^{\prime} \\
t_{b} & r_{b}^{\prime}
\end{array}\right)
$$

donde $r_{b}\left(t_{b}\right)$ y $r_{b}^{\prime}\left(t_{b}^{\prime}\right)$ son las amplitudes de reflexión (transmisión) cuando la incidencia en la barrera es desde el exterior y el interior de la cavidad, respectivamente (ver la figura 2.2). 


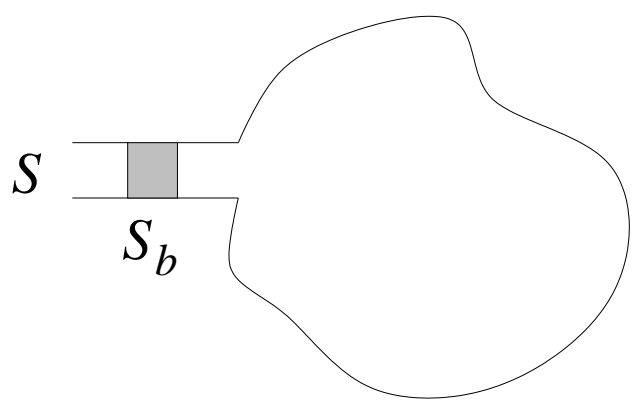

Figura 2.2: Cavidad caótica conectada a una guía de ondas. Entre la guía y la cavidad se coloca una barrera que produce que parte de las ondas se reflejen antes de ingresar a la cavidad.

En esta situación particular la matriz $S$ se puede escribir como la contribución de dos términos:

$$
S=r_{b}+S_{\text {fluc }}
$$

El primer término contiene la respuesta rápida del sistema debido a las reflexiones directas en la entrada de la cavidad. El segundo término contiene las reflexiones múltiples entre la barrera y la cavidad caótica cuyo promedio en el ensemble es nulo. Entonces, la matriz $S$ óptica está dada por $\langle S\rangle=r_{b}$. Las amplitudes $t_{b}$ y $t_{b}^{\prime}$ satisfacen

$$
t_{b}^{\dagger} t_{b}=\mathbf{1}_{N}-r_{b}^{\dagger} r_{b} \quad \text { y } \quad t_{b}^{\dagger \dagger} t_{b}^{\prime}=\mathbf{1}_{N}-r_{b} r_{b}^{\dagger} .
$$

\subsubsection{Absorción}

Existen situaciones en las que la condición de conservación de flujo no se satisface. Tal es el caso de sistemas ondulatorios clásicos como microondas u ondas elásticas. En esos casos la matriz de dispersión que describe el sistema, que denotamos como $\tilde{S}$, ya no es una matriz unitaria.

Uno de los modelos más conocidos para estudiar la absorción en cavidades caóticas es el modelo de la guía de onda ficticia. En este modelo la guía ficticia soporta $N_{p}$ canales ficticios o parásitos, cada uno de ellos acoplados a la cavidad en forma equivalente con una probabilidad de transmisión $T_{p}$. La matriz de dispersión $S$ que describe el sistema físico con la guía ficticia es unitaria y tiene la estructura

$$
S=\left(\begin{array}{cc}
\tilde{S} & S_{1 p} \\
S_{p 1} & S_{p p}
\end{array}\right)
$$



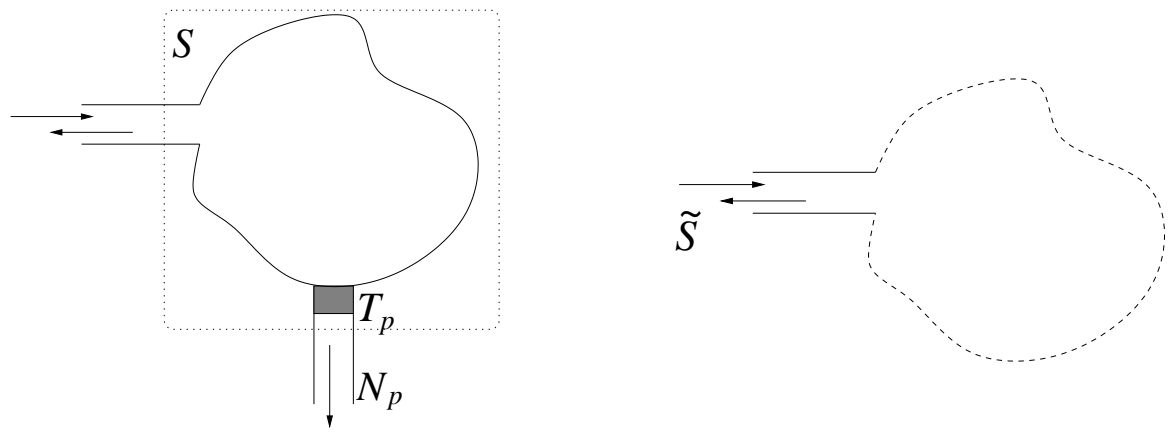

Figura 2.3: Cavidad caótica con una superguía que representa los $N$ canales abiertos. La guía de ondas con $N_{p}$ canales acoplados con probabilidad de transmisión $T_{p}$ en la figura de la izquierda es ficticia y representa las pérdidas o absorción. La figura de la derecha representa la situación física accesible en un experimento con absorción (la cavidad en línea discontinua intenta representar las pérdidas).

que contiene como submatriz a la matriz del sistema bajo estudio, $\tilde{S}$. La dimensión de $S$ es $N=N_{1}+N_{p}$, donde $N_{1}$ es la dimensión de la matriz $\tilde{S}$. $S_{1 p}$ y $S_{p 1}$ son matrices de $N_{1} \times N_{p}$ y $N_{p} \times N_{1}$ que conectan los modos de la guía real con los de la guía ficticia, mientras que $S_{p p}$ es de dimensión $N_{p} \mathrm{y}$ es de la forma $S_{p p}=\sqrt{1-T_{p}} \mathbf{1}_{N_{p}}$.

Dado que el modelo involucra un acoplamiento $T_{p}$ con los canales parásitos, el promedio sobre el ensemble de $S$ no es cero, de hecho es

$$
\langle S\rangle=\left(\begin{array}{cc}
0_{N_{1}} & 0 \\
0 & \sqrt{1-T_{p}} \mathbf{1}_{N_{p}}
\end{array}\right)
$$

por lo que su densidad de probabilidad está dada por la ecuación (2.15). Usando las propiedades generales de $S$ es posible determinar la densidad de probabilidad de $\tilde{S}$. Lo que sí es evidente de la ecuación (2.20) es que $\langle\tilde{S}\rangle=0$. Es decir, $\tilde{S}$ representa un sistema con absorción en ausencia de procesos directos.

\subsubsection{Absorción y procesos directos}

Un sistema en presencia de absorción y procesos directos se puede tratar de la misma manera que en la sección 2.5.2 una vez que $\tilde{S}$ es conocida (ver la figura 2.4). Es decir [41, 42],

$$
\tilde{S}=\tilde{r}_{b}+\tilde{S}_{\text {fluc }}
$$




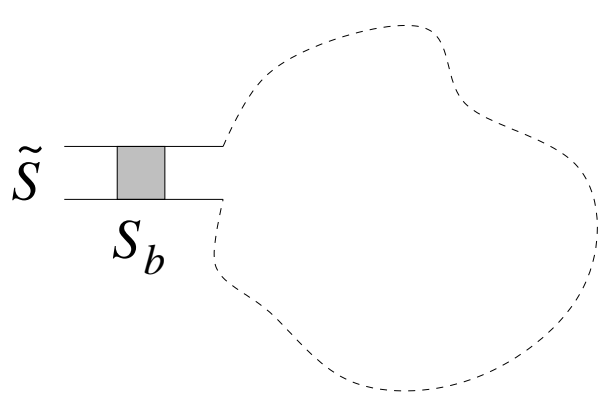

Figura 2.4: Cavidad en presencia de absorción y procesos directos.

En la búsqueda de nuestros objetivos, seguiremos las ideas expresadas en este capítulo y abordaremos los diferentes procesos que ocurren en los sistemas ondulatorios uno a la vez. Así, en el próximo capítulo analizaremos los efectos de la absorción y los procesos directos en un sistema simple que no involucra la complejidad del caos. 


\section{Capítulo 3}

\section{Dispersión en una cavidad elástica unidimensional}

En este capítulo estudiamos la dispersión de ondas en una cavidad unidimensional desde el punto de vista tanto teórico como experimental. En busca de un análogo clásico de un sistema cuántico dispersor unidimensional, proponemos un modelo teórico para la realización experimental de la cavidad en un sistema elástico en la que se hacen incidir ondas torsionales. En mecánica cuántica, este modelo corresponde con la dispersión por medio de un potencial tipo delta de Dirac localizado a una cierta distancia de un potencial penetrable. Al final comparamos las predicciones teóricas con los resultados experimentales.

\subsection{Propuesta experimental elástica}

En esta sección nuestro objetivo es estudiar un modelo clásico análogo de la cavidad unidimensional (1D) cuántica que consiste de un potencial tipo delta de Dirac localizado a una cierta distancia de una pared impenetrable [29]. Aunque este problema ha sido analizado resolviendo la ecuación de Schrödinger proponiendo un potencial complejo, para simular la disipación en el sistema, no había sido abordado desde el punto de vista de las ondas clásicas. Aquí consideramos la dispersión de ondas torsionales en una varilla elástica de sección transversal cuadrada. La cavidad se construye mediante el maquinado de una muesca rectangular situada a una cierta distancia de uno de los extremos de la varilla, que sirve como un elemento dispersor local; resolvemos el problema por medio del formalismo de la matriz de dispersión. Este modelo nos permite hacer una propuesta experimental con la 
cual verificar nuestras predicciones teóricas.

\subsubsection{Elemento dispersor con absorción}

Supongamos ondas torsionales de frecuencia $f$ que se propagan a lo largo de una varilla elástica de sección transversal cuadrada de lado $W$, a una velocidad $v$. La varilla tiene un elemento dispersor que consiste de una muesca de ancho $a$ y profundidad $h_{0}$ como se muestra en la figura 3.1. Supondremos que la muesca contiene un material disipativo que absorbe las ondas que llegan ahí. En el caso unidimensional, a bajas frecuencias, la ecuación que describe las ondas torsionales en la varilla cuyo eje se encuentra a lo largo del eje $x$, está dada por

$$
\frac{\partial^{2} \chi(x, t)}{\partial x^{2}}-\frac{1}{v^{2}} \frac{\partial^{2} \chi(x, t)}{\partial t^{2}}=0
$$

que en el caso estacionario donde $\chi(x, t)=\varphi(x) \mathrm{e}^{-\mathrm{i} 2 \pi f t}$ se reduce a la ecuación de Helmholtz

$$
\frac{\mathrm{d}^{2} \varphi(x)}{\mathrm{d} x^{2}}+k^{2} \varphi(x)=0
$$

donde $k$ es el número de onda dado por $k=2 \pi f / v$.

La velocidad de la ondas torsionales en una varilla de sección transversal rectangular es distinta en cada región pues depende de factores geométricos. Para una varilla de ancho $W$ y altura $h_{j}$ la velocidad $v_{j}, v_{0}$ y $v$ para las regiones dentro y fuera de la muesca, respectivamente, está dada por

$$
v_{j}=\sqrt{\frac{G}{\rho} \frac{\alpha_{j}}{I_{j}}}
$$

donde $G$ es el módulo de corte y $\rho$ es la densidad de la varilla; $\alpha_{j}$ es la constante de torsión que está dada por la serie de Navier

$$
\alpha_{j}=\frac{256}{\pi^{6}} \sum_{p=0}^{\infty} \sum_{q=0}^{\infty} \frac{1}{(2 p+1)^{2}(2 q+1)^{2}} \frac{h_{j} W_{j}}{\left(\frac{2 p+1}{h_{j}}\right)^{2}+\left(\frac{2 q+1}{W_{j}}\right)^{2}}
$$

e $I_{j}$ es el momento polar de inercia. En las regiones fuera de la muesca el momento de inercia es

$$
I=\frac{W^{4}}{6}
$$

mientras que en la región de la muesca es

$$
I_{0}=\frac{W^{4}}{6}\left(2 \frac{h_{0}^{3}}{W^{3}}-3 \frac{h_{0}^{2}}{W^{2}}+2 \frac{h_{0}}{W}\right) .
$$




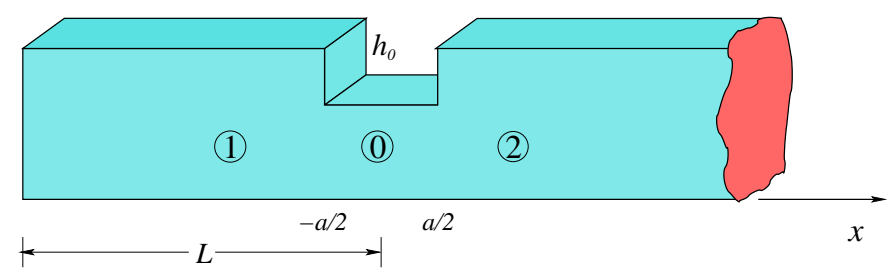

Figura 3.1: Vista de la varilla elástica con una muesca de ancho a y profundidad $h_{0}$. Las diferentes regiones se indican con 0,1 y 2. La región 2 se extiende hasta infinito.

Esto implica que el número de onda sea distinto en cada región. Fuera de la muesca el número de onda $k$, está dado por

$$
k=\frac{2 \pi}{v} f
$$

mientras que para la región de la muesca el número de onda es de la forma $\tilde{k}=k_{0}+\mathrm{i} \kappa_{0}$, donde $k_{0}$ es el número de onda que se tendría en ausencia de absorción y $\kappa_{0}$ está relacionada con el término disipativo, $\Gamma$, propuesto en la relación de dispersión que se obtiene de la ecuación (3.2) dada por

$$
\tilde{k}^{2}=\left(\frac{2 \pi f}{v_{0}}\right)^{2}+\mathrm{i} \Gamma^{2}
$$

De esta expresión podemos obtener que

$$
\begin{aligned}
& k_{0}=\sqrt{\frac{1}{2}\left(\frac{2 \pi f}{v_{0}}\right)^{2}+\frac{1}{2} \sqrt{\left(\frac{2 \pi f}{v_{0}}\right)^{4}+\Gamma^{4}}} \\
& \kappa_{0}=\sqrt{-\frac{1}{2}\left(\frac{2 \pi f}{v_{0}}\right)^{2}+\frac{1}{2} \sqrt{\left(\frac{2 \pi f}{v_{0}}\right)^{4}+\Gamma^{4}}} .
\end{aligned}
$$

La solución a la ecuación (3.2) en las diferentes regiones de la varilla, se pueden escribir como

$$
\varphi(x)= \begin{cases}A_{1} \mathrm{e}^{\mathrm{i} k x}+B_{1} \mathrm{e}^{-\mathrm{i} k x} & \text { para } x \leq-a / 2 \\ A_{0} \mathrm{e}^{\mathrm{i} \tilde{k} x}+B_{0} \mathrm{e}^{-\mathrm{i} \tilde{k} x} & \text { para }-a / 2 \leq x \leq a / 2 \\ A_{2} \mathrm{e}^{-\mathrm{i} k x}+B_{2} \mathrm{e}^{\mathrm{i} k x} & \text { para } x \geq a / 2\end{cases}
$$

tomando como origen el centro de la muesca. Aquí, $A_{j}$ y $B_{j}$ son las amplitudes de las ondas en las diferentes regiones. 
Las condiciones de frontera a través de la continuidad de la función $\varphi(x)$ y la conservación del momento de torsión dado por

$$
M_{T_{j}}=G \alpha_{j} \frac{\partial \varphi_{j}}{\partial x}
$$

conducen a un conjunto de ecuaciones que relacionan las amplitudes de las ondas salientes con las correspondientes ondas entrantes, que se pueden escribir en forma matricial como

$$
\left(\begin{array}{c}
B_{1} \mathrm{e}^{\mathrm{i} k a / 2} \\
B_{2} \mathrm{e}^{\mathrm{i} k a / 2}
\end{array}\right)=\tilde{S}_{0}\left(\begin{array}{c}
A_{1} \mathrm{e}^{-\mathrm{i} k a / 2} \\
A_{2} \mathrm{e}^{-\mathrm{i} k a / 2}
\end{array}\right)
$$

donde $\tilde{S}_{0}$ es la matriz de dispersión de la muesca absorbente y tiene la estructura

$$
\tilde{S}_{0}=\left(\begin{array}{cc}
\tilde{r}_{0} & \tilde{t}_{0} \\
\tilde{t}_{0} & \tilde{r}_{0}
\end{array}\right)
$$

con $\tilde{r}_{0}$ y $\tilde{t}_{0}$ las amplitudes de reflexión y transmisión, respectivamente, las cuales están dadas por

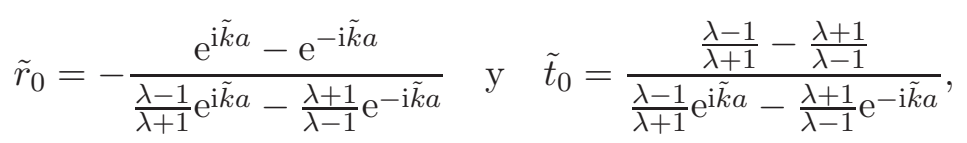

donde $\lambda=\eta \tilde{k} / k$ con $\eta=\alpha_{0} / \alpha$.

\subsubsection{Modelo elástico de la cavidad}

Para construir la cavidad colocamos la muesca a una cierta distancia del extremo de una varilla semi-infinita, como se muestra en la figura 3.1.

Dado que el extremo de la varilla está libre la condición en la frontera es de Neumann, de manera que la derivada de $\varphi(x)$ en $x=-L$ se anula; así, de la ecuación (3.11), tenemos que

$$
B_{1}=A_{1} \mathrm{e}^{-2 \mathrm{i} k L} .
$$

La matriz de dispersión $\tilde{S}$ que describe la dispersión de las ondas por la cavidad se obtiene combinando las ecuaciones (3.13) y (3.16) para obtener

$$
B_{2} \mathrm{e}^{\mathrm{i} k a / 2}=\tilde{S} A_{2} \mathrm{e}^{-\mathrm{i} k a / 2}
$$

donde

$$
\tilde{S}=\tilde{r}_{0}+\tilde{t}_{0} \frac{1}{1-\tilde{r}_{0} \mathrm{e}^{2 \mathrm{i} k(L-a / 2)}} \mathrm{e}^{2 \mathrm{i} k(L-a / 2)} \tilde{t}_{0} .
$$



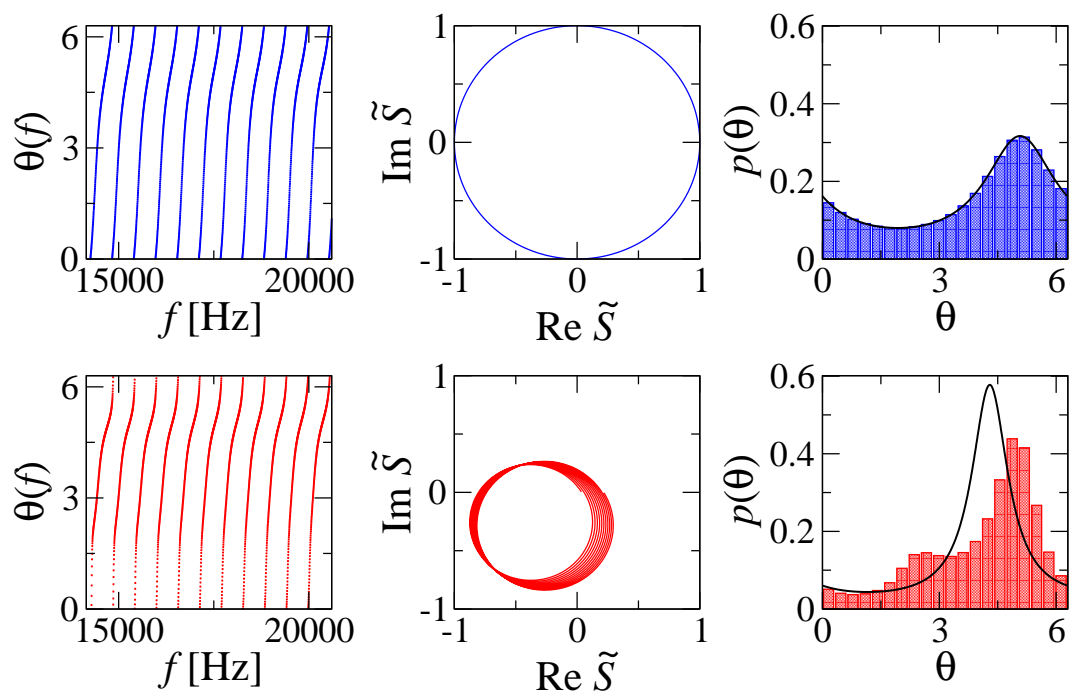

Figura 3.2: Fase $\theta(f)$ de la matriz $\tilde{S}$ en el rango de frecuencias de $14 \mathrm{kHz}$ a $20 \mathrm{kHz}$ (izquierda). Movimiento de $\tilde{S}$ en el plano de Argand (centro) como función de la frecuencia. Distribución de $\theta(f)$ (histograma) comparada con el kernel de Poisson de la ecuación (3.19) (línea continua de las figuras en la parte derecha). Las figuras en la parte superior corresponden al caso ideal sin absorción, $\Gamma=0$, mientras que las de la parte inferior corresponden al caso con absorción con $\Gamma=800$.

Esta ecuación tiene una interpretación física muy clara. El primer término del lado derecho representa la reflexión en la muesca de la onda torsional que llega libremente desde la derecha. El segundo término representa las reflexiones múltiples entre la muesca y el extremo de la varilla para después salir. Las ondas salientes ya no regresan a la cavidad por ser un medio semiinfinito.

La matriz de dispersión $\tilde{S}$ de la cavidad, dada en la ecuación (3.18), depende de la frecuencia a través de los números de onda $k$ y $\tilde{k}$, además de los parámetros físicos de la varilla. En una situación realista, donde existe absorción, $\tilde{S}(f)=\sqrt{R_{0}} \mathrm{e}^{\mathrm{i} \theta(f)}$, cuyo movimiento como función de $f$ describe un círculo de radio $\sqrt{R_{0}}$ en el plano de Argand; $\tilde{S}(f)$ no visita este círculo de manera uniforme sino que se distribuye de acuerdo al kernel de Poisson no unitario [29],

$$
p(\theta)=\frac{1}{2 \pi} \frac{R_{0}-|\overline{\tilde{S}}|^{2}}{|\tilde{S}-\overline{\tilde{S}}|^{2}},
$$



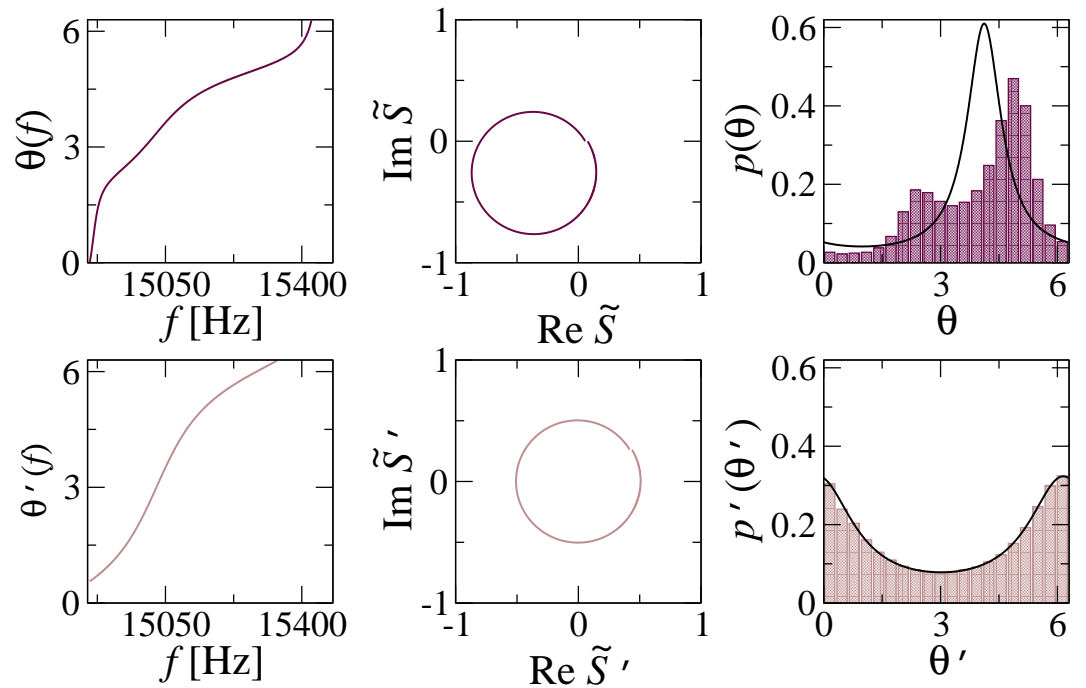

Figura 3.3: Matriz $\tilde{S}$ de una resonancia alrededor de $15000 \mathrm{~Hz}$ (parte superior) para $\Gamma=800$. En la parte inferior se muestra el comportamiento de la matriz de dispersión vista desde el origen del círculo que describe en el plano de Argand. Por esta razón indicamos con una prima a la matriz $\tilde{S}$. Se observa un buen acuerdo con el kernel de Poisson pese a la absorción.

donde $\overline{\tilde{S}}$ es el promedio en la frecuencia de $\tilde{S}(f)$, el cual junto con $R_{0}$, se obtienen del experimento. Notemos que en esta situación ideal sin absorción, $R_{0}=1$ y la ecuación (3.19) se reduce a la expresión del kernel tradicional [43].

Consideremos el caso de una varilla de aluminio de sección transversal cuadrada, de lado $W=25.4 \mathrm{~mm}$, con una muesca de ancho $a=0.9 \mathrm{~mm} \mathrm{y}$ profundidad $h_{0}=18.0 \mathrm{~mm}$. Los parámetros físicos de la aleación de aluminio 6061-T6 son: $G=26$ GPa y $\rho=2.7 \mathrm{~g} / \mathrm{cm}^{3}$, tal que $\sqrt{G / \rho}=3103.2 \mathrm{~m} / \mathrm{s}$. Para el caso sin absorción [ $\Gamma=0$ en la ecuación (3.8)], en el rango de frecuencias de $14 \mathrm{kHz}$ a $20 \mathrm{KHz}$ obtenemos 11 resonancias que se muestran en la figura 3.2 en color azul. Podemos observar que en este rango de frecuencias las propiedades del sistema no varían mucho; es decir, el sistema es estacionario. En la misma figura mostramos el movimiento de $\tilde{S}$, que describe un círculo de radio constante centrado en el origen, y comparamos la distribución de la fase $\theta$ de las 11 resonancias con el kernel de Poisson, ecuación (3.19) con $R_{0}=1$ (línea continua); observamos un acuerdo excelente. Para el caso con absorción $(\Gamma=800)$ las gráficas en color rojo en la 
figura 3.2 muestran el comportamiento de la fase y el movimiento de $\tilde{S}$ como

función de la frecuencia; $\tilde{S}$ ya no describe un círculo sino una espiral. La distribución de la fase ya no concuerda con el kernel de Poisson.

El hecho de que en presencia de absorción el kernel no describe la distribución de la fase es debido a que el sistema deja de ser estacionario al considerar el conjunto de 11 resonancias. Esto lo podemos demostrar analizando las resonancias individualmente. En ese caso, el kernel de Poisson se satisface para la distribución de la fase cuando se observa el círculo desde su centro, como se observa en la parte inferior de la figura 3.3.

La realización experimental de esta propuesta constituyó la Tesis de maestría de Marcel Cobián Suárez en el Posgrado en Ciencia e Ingeniería de Materiales de la Unidad Azcapotzalco de esta Universidad (ver la referencia [44]). Tanto la propuesta como el análisis y la interpretación de los resultados del experimento forman parte de la contribución del autor de la presente Tesis. Las conclusiones que se extraen del análisis son relevantes para los objetivos que nos planteamos sobre el efecto de la absorción y los procesos directos.

\subsection{Realización experimental}

\subsection{1. $\quad$ El montaje experimental}

En la figura 3.4 se muestra el montaje usado para la realización del experimento [44]. El objetivo consistió en simular la varilla semiinfinita, lo cual se logró a través de la construcción de un sistema mecánico pasivo de eliminación de patrones estacionarios (región IV en la figura 3.4).

La generación de las ondas torsionales en el sistema se logra por medio de una configuración adecuada de transductores electromagnético acústicos, o EMATs, por sus siglas en inglés [16]. La respuesta del sistema que se produce por esta excitación se detecta con un segundo EMAT localizado justo afuera de la cavidad, como se muestra en la figura 3.4. Para producir la vibración torsional en el sistema se usa un analizador de redes vectorial (VNA por sus siglas en inglés) que produce una señal sinusoidal de frecuencia $f$, que se envía al EMAT excitador. La aceleración torsional que mide el EMAT detector se registra en el VNA para su análisis posterior [45, 46].

\subsubsection{Resultado del experimento}

El espectro de frecuencias, medido tanto para la magnitud como para la fase de la señal reflejada se muestra en la figura 3.5 para el rango de fre- 


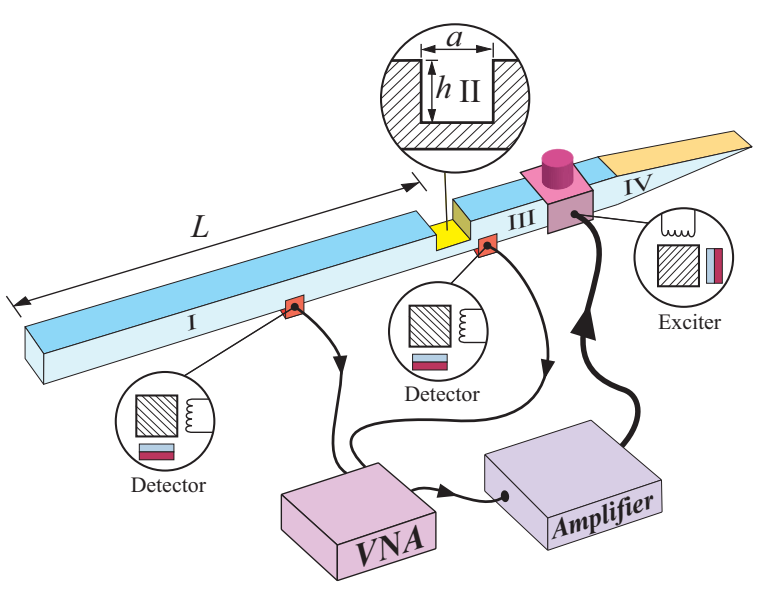

Figura 3.4: En el sistema $L=2.5 \mathrm{~m}$. La profundidad y ancho de la muesca son $p=0.018$ $\mathrm{m}$ y $a=0.0009 \mathrm{~m}$, respectivamente. La varilla tiene una longitud total de $3.6 \mathrm{~m}$ y una sección transversal cuadrada de $W=0.0254 \mathrm{~m}$ de lado. La cuña tiene una longitud de $0.40 \mathrm{~m}$ y está cubierta de una espuma polimérica. La varilla elástica está suspendida por dos hilos de nylon (no se muestran en la figura). La conección del equipo se muestra en la parte inferior.

cuencias entre $14 \mathrm{kHz}$ y $20 \mathrm{kHz}$. En este intervalo de frecuencias se observan muchas resonancias, algunas de las cuales pertenecen a los modos torsionales. Con la ayuda del modelo teórico identificamos las que corresponden sólo a ese tipo de modos, que se indican con segmentos verticales. La magnitud y fase de esos modos los interpretamos como el módulo y fase de la matriz de dispersión no normalizada [44]; esto es,

$$
S(f)=\sqrt{R(f)} \mathrm{e}^{\mathrm{i} \theta(f)} .
$$

En la figura 3.6 podemos observar dos de las resonancias torsionales del espectro de frecuencias, una en (a) $14819 \mathrm{~Hz}$ y la otra en (b) $15960 \mathrm{~Hz}$. En los paneles (c) y (d) observamos que las resonancias describen círculos de radio menor que uno en el plano de Argand, cuyo centro se encuentra desplazado del origen y sobre la curva de impedancia. Visto desde el centro del círculo el módulo y fase se describen por

$$
S^{\prime}(f)=\sqrt{R_{0}} \mathrm{e}^{\mathrm{i} \theta^{\prime}(f)} .
$$

En los paneles de la columna derecha de la figura 3.6 comparamos los histogramas de las correspondientes fases medidas desde el centro de cada círculo 

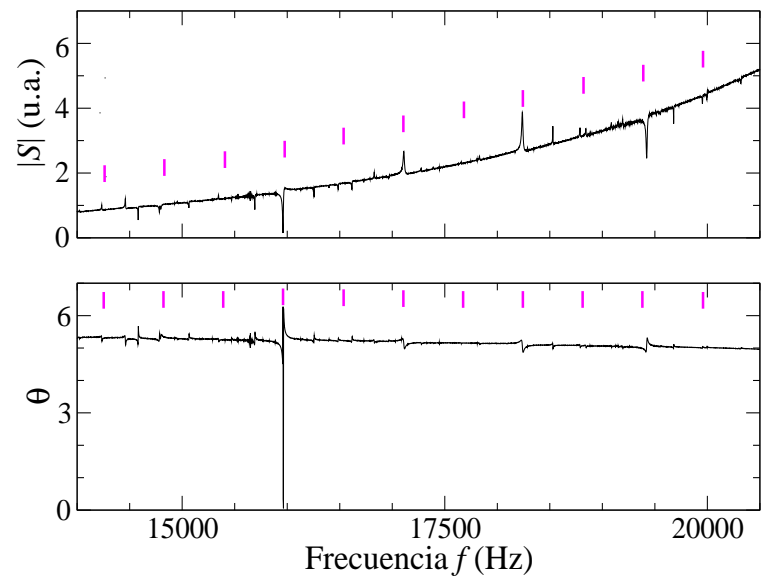

Figura 3.5: Espectro de resonancias, en magnitud y fase de $S(f)$. Los segmentos verticales corresponden a las predicciones teóricas. Figura tomada de las referencias [44, 46].

con el kernel de Poisson de la ecuación (3.19). En cada caso, tomamos el radio promedio del círculo y $\overline{S^{\prime}(f)}$ de los datos experimentales. Como podemos ver, el acuerdo es excelente, aún para la resonancia en $f=14819 \mathrm{~Hz}$ que muestra el peor acuerdo [45].

De acuerdo con las predicciones teóricas de la sección anterior, estos resultados muestran que la absorción no juega un papel relevante en el sistema elástico considerado ${ }^{1}$. Cualquier efecto debido a la absorción queda oculto en las unidades arbitrarias del experimento. La cantidad que juega un papel relevante en el experimento es $\overline{S^{\prime}(f)}$ que describe los procesos directos.

\subsubsection{Un nuevo proceso directo}

En la figura 3.5 también se puede observar que las resonancias se encuentran sobre una curva base de impedancia debido a los componentes electromagnéticos de los dispositivos de medición y excitación. Esto lo verificamos por medio de una medición sin producir vibración torsional alguna, lo que se conoce como hipótesis nula. Debido a esta impedancia los círculos descritos por cada resonancia se encuentran sobre la curva base, desplazados del origen. Por lo tanto, la matriz de dispersión del sistema consiste de dos partes: de la matriz de dispersión vista desde el centro más su desplazamiento a lo

\footnotetext{
${ }^{1}$ Por esta razón denotaremos a la matriz de dispersión sin tilde, $S$.
} 

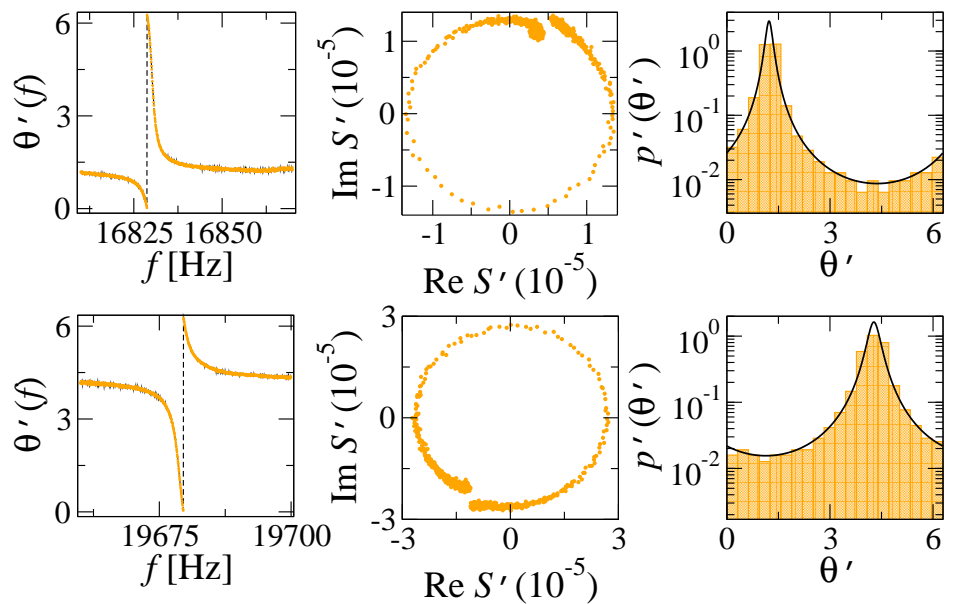

Figura 3.6: Fase de $S^{\prime}$ como función de la frecuencia $f$ para la resonancia en $16830 \mathrm{~Hz}$ (renglón superior) y en $19680 \mathrm{~Hz}$ (renglón inferior). Movimiento de $S^{\prime}$ en el plano de Argand (columna de enmedio). Las distribuciones de la fase (columna derecha) muestran un acuerdo excelente con el kernel de Poisson no unitario (línea continua).

largo de la curva de impedancia. Esto es,

$$
S(f)=S^{\prime}(f)+Z
$$

donde $Z$ son las coordenadas del centro del círculo descrito por $S^{\prime}(f)$ en el plano de Argand. Esto implica que la matriz $S$ óptica, $S_{\text {opt }} \equiv \overline{S(f)}$, también consta de dos partes, la respuesta directa en la dispersión de ondas debida a la muesca, que es de naturaleza mecánica y una respuesta rápida electromagnética adicional que viene de utilizar los EMATs en el proceso de medición. Es decir,

$$
S_{\mathrm{opt}}=\overline{S^{\prime}}+Z
$$

Una correcta descripción del proceso de dispersión debe tomar en cuenta este nuevo proceso directo.

\subsection{Una nueva resonancia de Fano}

En la figura 3.7 mostramos el movimiento de $S(f)$ en el plano de Argand, como función de la frecuencia. Como se espera, para cada resonancia 


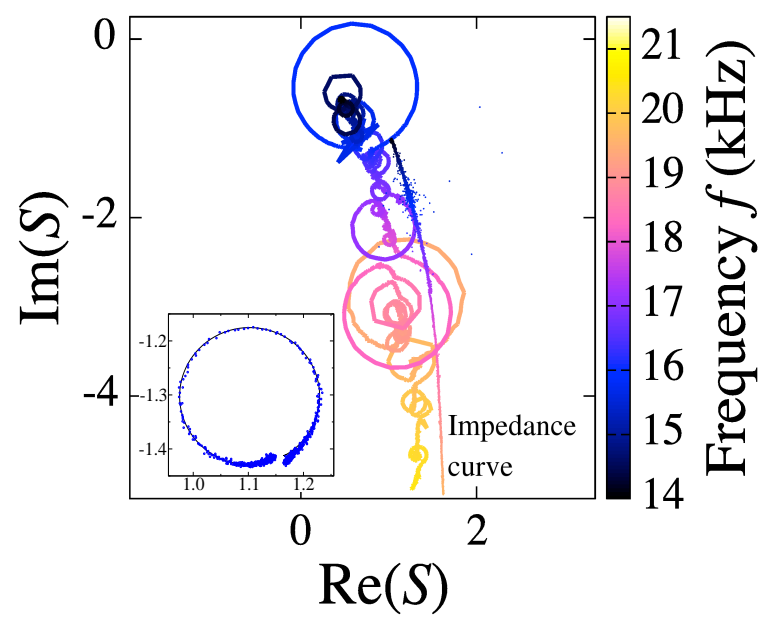

Figura 3.7: Movimiento de $S(f)$ en el plano de Argand como función de la frecuencia $f$ para las mismas resonancias de la figura 3.5. En línea gruesa se muestra la curva de impedancia, medida sin excitar las vibraciones torsionales. Figura interna: resonancia en $f_{\text {res }}=14819 \mathrm{~Hz}$ comparada con la ecuación (3.24) en línea negra continua [46].

$S(f)$ describe un círculo que se encuentra sobre la curva de impedancia ${ }^{2}$. Como se puede ver, estos círculos no cierran totalmente debido a la impedancia electromagnética que varía ligeramente con la frecuencia. Esto es, el proceso directo que viene de la medición a través de los componentes electromagnéticos da lugar a una asimetría adicional al perfil de la resonancia del tipo Fano, que es de la forma

$$
S(f)=a+b \frac{2\left(f-f_{\mathrm{res}}\right)-q \Gamma}{2\left(f-f_{\mathrm{res}}\right)-\mathrm{i} \Gamma} .
$$

Un ajuste de la ecuación (3.24) para la resonancia en $f_{\text {res }}=14819 \mathrm{~Hz}$ se encuentra la gráfica interna de la figura 3.7, que se muestra en módulo y fase en la figura 3.8. Los valores correspondientes de los parámetros de la ecuación (3.24) son el ancho $\Gamma=2.2 \mathrm{~Hz}, a=(8.08+\mathrm{i} 1.23) \times 10^{-5}$, $b=(-3.45+\mathrm{i} 15.42) \times 10^{-5}$, y el parámetro complejo de Fano es $q=$ $0.030+\mathrm{i} 0.842$.

\footnotetext{
${ }^{2}$ La curva paralela que se observa en la figura 3.7 corresponde a la que se obtiene con la hipótesis nula.
} 

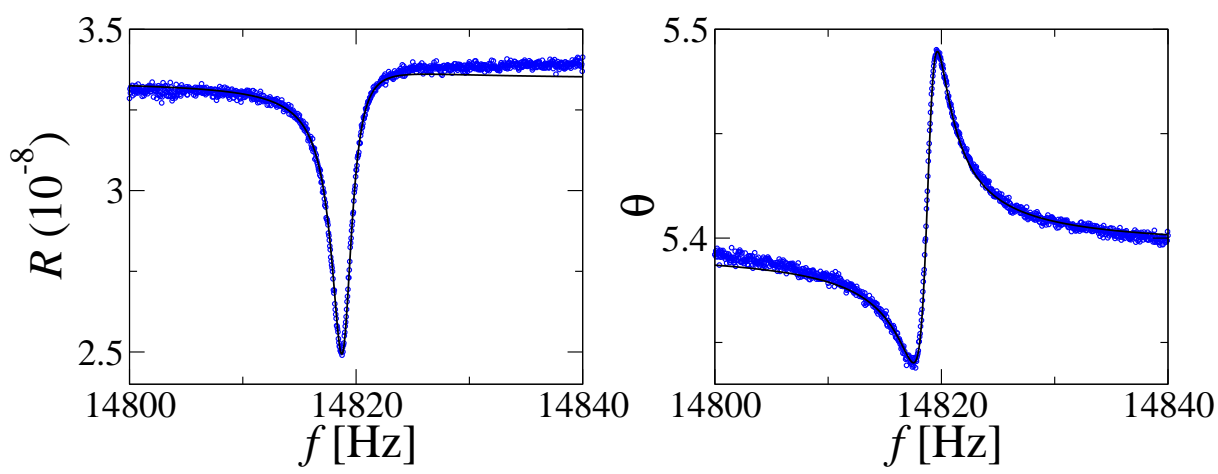

Figura 3.8: Ajuste de la ecuación (3.24) en línea continua con la resonancia medida en $f_{\text {res }}=14819 \mathrm{~Hz}$, en módulo y fase. Los valores de los parámetros son $\Gamma=2.2 \mathrm{~Hz}$, $a=(8.08+\mathrm{i} 1.23) \times 10^{-5}, b=(-3.45+\mathrm{i} 15.42) \times 10^{-5}$ y $q=0.030+\mathrm{i} 0.842[46]$.

\subsubsection{Distribución de la resonancia}

Como mostramos en la sección anterior, la matriz de dispersión de cada resonancia vista desde su propio centro, $S^{\prime}(f)$, no visita la circunferencia de una manera uniforme sino que lo hace de acuerdo con el kernel de Poisson

$$
P^{\prime}\left(S^{\prime}\right)=\frac{1}{2 \pi} \frac{R^{\prime}-\left|\overline{S^{\prime}}\right|^{2}}{\left|S^{\prime}-\overline{S^{\prime}}\right|^{2}} \delta\left(R^{\prime}-R_{0}\right),
$$

donde $R_{0}$ es el radio del círculo (constante), $\overline{S^{\prime}} \equiv \overline{S^{\prime}(f)}$ es el promedio sobre la frecuencia de $S^{\prime}(f)$, determinada del experimento.

Para conocer cómo se distribuye la matriz $S$ debemos tomar en cuenta el proceso directo de la impedancia electromagnética. Esto se puede hacer a través de la relación

$$
P(S)=J\left(S^{\prime}(S)\right) P^{\prime}\left(S^{\prime}(S)\right),
$$

donde $J\left(S^{\prime}(S)\right)$ es el Jacobiano de la transformación dada por la ecuación (3.22); en este caso $J\left(S^{\prime}(S)\right)=1$. Esto es,

$$
P(S)=\frac{1}{2 \pi} \frac{|S-Z|^{2}-\left|S_{\mathrm{opt}}-Z\right|^{2}}{\left|S-S_{\mathrm{opt}}\right|^{2}} \delta\left(|S-Z|^{2}-R_{0}\right) .
$$

Es importante mencionar que la distribución queda completamente determinada por dos parámetros que se obtienen del experimento, la matriz $S_{\mathrm{opt}}$ y $Z$. Esto contrasta con el caso en ausencia de impedancia donde el único parámetro libre que determina la distribución es $S_{\text {opt }}$ [43]. 

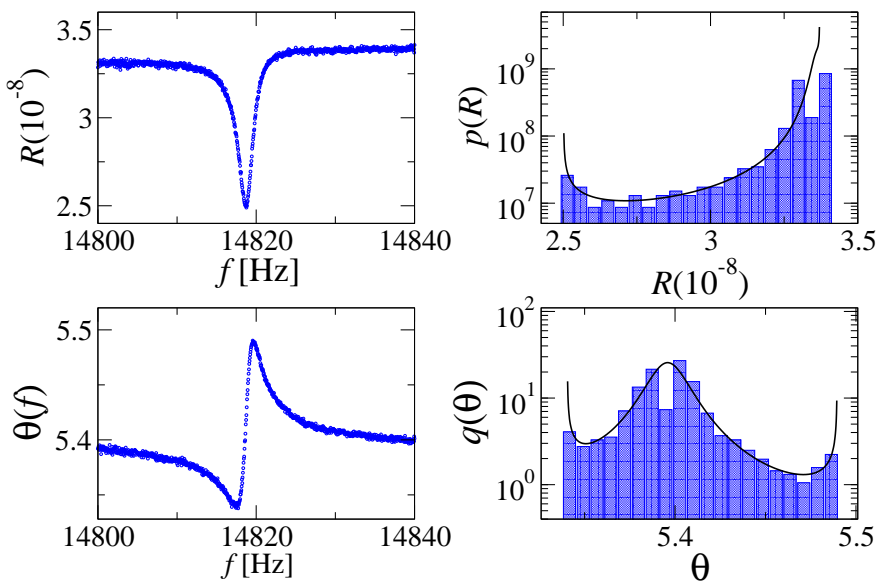

Figura 3.9: Resonancia en $14819 \mathrm{~Hz}$. A la izquierda se muestran la magnitud al cuadrado y la fase de $S(f)$. A la derecha se muestra la comparación de los histogramas de los datos experimentales con las expresiones teóricas, ecuaciones (3.29) y (3.32), en línea continua para $R_{0}=1.6187 \times 10^{-10}, Z=(1.104-\mathrm{i} 1.304) \times 10^{-4}$ y $S_{\mathrm{opt}}=(1.148-\mathrm{i} 1.400) \times 10^{-4}[46]$.

Para visualizar la distribución de $S$ determinaremos las distribuciones marginales de $R$ y $\theta$, lo cual se puede hacer por integración directa (ver Apéndice B). Para obtener la distribución marginal de $R$ integramos la ecuación (3.27) con respecto a $\theta$; es decir

$$
p(R)=\int_{0}^{2 \pi} P(S) \mathrm{d} \theta
$$

cuyo resultado es

$$
\begin{aligned}
& p(R)=\frac{1}{2 \pi} \frac{R-\left|S_{\mathrm{opt}}-Z\right|^{2}}{\sqrt{\left[R-\left(|Z|-\sqrt{R_{0}}\right)^{2}\right]\left[\left(|Z|+\sqrt{R_{0}}\right)^{2}-R\right]}}
\end{aligned}
$$

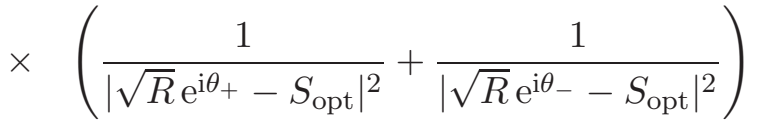

$$
\begin{aligned}
& \times \quad\left[\Theta\left(|Z|+\sqrt{R_{0}}-\sqrt{R}\right)+\Theta\left(\sqrt{R}-|Z|+\sqrt{R_{0}}\right)\right],
\end{aligned}
$$

donde $\Theta(z)$ es la función de Heaviside de $z$ y

$$
\begin{aligned}
& \sqrt{R} \mathrm{e}^{\mathrm{i} \theta_{ \pm}}=\frac{1}{2 Z^{*}}\left\{\left(R+|Z|^{2}-R_{0}\right)\right. \\
& \left. \pm \mathrm{i} \sqrt{\left[R-\left(|Z|-\sqrt{R_{0}}\right)^{2}\right]\left[\left(|Z|+\sqrt{R_{0}}\right)^{2}-R\right]}\right\} .
\end{aligned}
$$



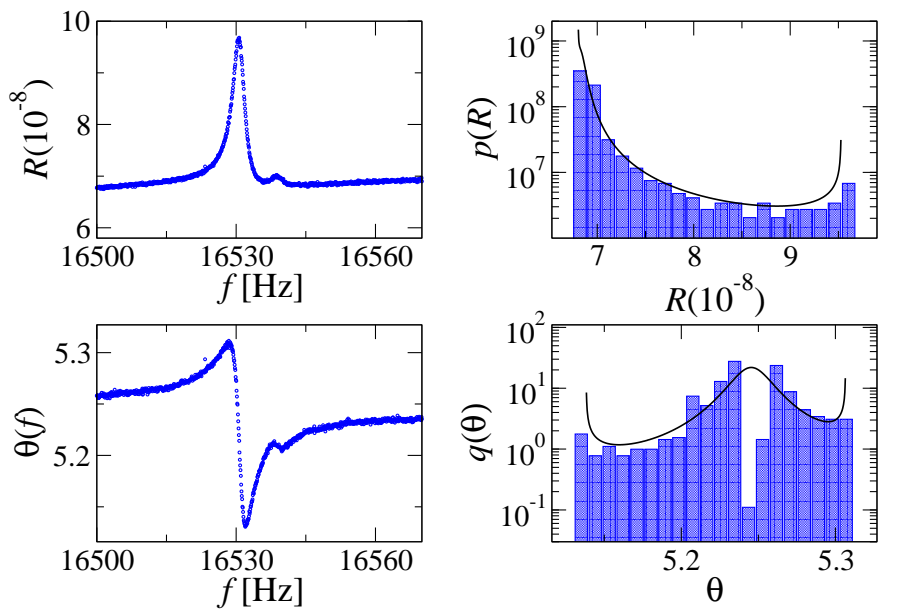

Figura 3.10: Resonancia en $16530 \mathrm{~Hz}$. Observamos un excelente acuerdo entre la teoría y el experimento, a pesar de la aparición de un segundo tipo de resonancia que no fué completamente eliminado por los absorbedores. Aquí, $R_{0}=5.7327 \times 10^{-10}$, $Z=(1.390-\mathrm{i} 2.485) \times 10^{-4}$ y $S_{\mathrm{opt}}=(1.338-\mathrm{i} 2.289) \times 10^{-4}[46]$.

Es claro que los valores mínimo y máximo de $\sqrt{R}$ están limitados por $Z$; es decir, el mínimo (máximo) está dado por la suma de la distancia al centro $|Z|$ menos (más) el radio del círculo $\sqrt{R_{0}}$.

De manera similar, la distribución marginal para la parte angular se obtiene mediante la integración de la ecuación (3.27) sobre $R$; es decir

$$
q(\theta)=\int_{0}^{1} P(S) \mathrm{d} R
$$

cuyo resultado es

$$
\begin{aligned}
& q(\theta)=\frac{1}{2 \pi} \frac{R_{0}-\left|S_{\mathrm{opt}}-Z\right|^{2}}{\sqrt{R_{0}-\left[\operatorname{Im}\left(Z^{*} \mathrm{e}^{\mathrm{i} \theta}\right)\right]^{2}}} \\
& \times\left(\frac{\sqrt{R_{+}}}{\left|\sqrt{R_{+}} \mathrm{e}^{\mathrm{i} \theta}-S_{\mathrm{opt}}\right|^{2}}+\frac{\sqrt{R_{-}}}{\mid \sqrt{R_{-}} \mathrm{e}^{\mathrm{i} \theta-\left.S_{\mathrm{opt}}\right|^{2}}}\right) \\
& \times\left[\Theta\left(\phi-\sin ^{-1} \frac{\sqrt{R_{0}}}{|Z|}-\theta\right)+\Theta\left(\theta-\phi-\sin ^{-1} \frac{\sqrt{R_{0}}}{|Z|}\right)\right],
\end{aligned}
$$

donde $\phi$ es la fase de $Z\left(Z=|Z| \mathrm{e}^{\mathrm{i} \phi}\right)$ y

$$
\sqrt{R_{ \pm}}=\operatorname{Re}\left(Z^{*} \mathrm{e}^{\mathrm{i} \theta}\right) \pm \sqrt{R_{0}-\left[\operatorname{Im}\left(Z^{*} \mathrm{e}^{\mathrm{i} \theta}\right)\right]^{2}} .
$$


En las figuras 3.9 y 3.10 mostramos los resultados de dos resonancias experimentales típicas, en 14819 y en $16530 \mathrm{~Hz}$. Los paneles de la izquierda de ambas figuras muestran la magnitud al cuadrado de $(R)$ y la fase $(\theta)$ de la amplitud de la señal medida; aquí notamos el efecto de la impedancia. En los paneles de la derecha se muestran las distribuciones de $R$ y $\theta$ como histogramas y se comparan con las expresiones teóricas de las distribuciones marginales (líneas continuas), ecuaciones (3.29) y (3.32). Los parámetros relevantes, $S_{\text {opt }}$ y $Z$, se obtuvieron de los datos experimentales. A pesar del hueco que aparecen en los histogramas de la fase, observamos un acuerdo excelente. Este hueco se debe a la falta de datos, ya que las resonancias experimentales no cierran por completo debido al efecto Fano de ambas clases de procesos directos. En la figura 3.10 se puede observar que aparece otra resonancia, alrededor de $16540 \mathrm{~Hz}$. El efecto de esta resonancia, que pertenece a otro tipo de vibración y que no fué eliminada completamente por el sistema pasivo de absorción de vibraciones, no afecta a las distribuciones de manera significativa. 


\section{Capítulo 4}

\section{Fluctuaciones universales del transporte electrónico}

En el capítulo anterior vimos que la absorción no afecta de manera significativa a las propiedades de la dispersión por una cavidad elástica, mientras que los procesos directos resultaron ser las cantidades más importantes para la descripción de la dispersión. Por tanto, en este capítulo abordaremos el problema del transporte a través de cavidades bidimensionales en ausencia de absorción. También evitaremos los procesos directos pero incorporaremos el ingrediente de la complejidad por medio de una dinámica clásica caótica debido a la geometría del sistema.

\subsection{Transporte electrónico y dispersión}

En el formalismo de Landauer-Büttiker el transporte electrónico a través de un conductor conectado a varios reservorios electrónicos, puestos a potenciales químicos $\mu_{i}$ fijos, por medio de guías electrónicas que soportan $N_{i}$ modos transversales de propagación, se puede reducir a un problema de dispersión. En un sistema de muchos puertos (un ejemplo se muestra en la figura 4.1) el flujo de corriente $I_{i}$ a través de la $i$-ésima guía, está dada por $[47]$

$$
I_{i}=\frac{e}{h}\left[\left(N_{i}-R_{i i}\right) \mu_{i}-\sum_{j(\neq i)} T_{i j} \mu_{j}\right],
$$

donde $e$ es la carga del electrón y $h$ es la constante de Planck. Aquí,

$$
T_{i j}=\sum_{m \in i} \sum_{n \in j}\left|S_{m n}\right|^{2} \quad \text { y } \quad R_{i i}=\sum_{m \in i} \sum_{n \in i}\left|S_{m n}\right|^{2}
$$




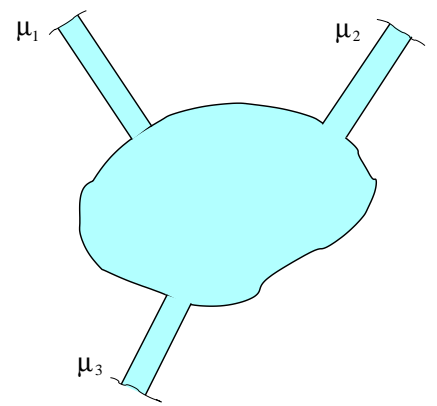

Figura 4.1: Esquema de un conductor de tres puertos o terminales.

son el coeficiente de transmisión desde desde la guía $j$ a la $i$ y el coeficiente de reflexión en la guía $i$, respectivamente ${ }^{1}$, y $S_{i j}$ son los elementos de la matriz de dispersión del sistema.

\subsection{Conductancia de un dispositivo electrónico de dos terminales}

\subsubsection{Fórmula de Landauer}

Para un sistema de dos terminales como el de la figura 4.2, la corriente en cada una de las guías es tal que $I_{1}=-I_{2} \equiv I$, donde

$$
I=\frac{e}{h}\left[\left(N_{1}-R_{11}\right) \mu_{1}-T_{12} \mu_{2}\right] .
$$

Usando la condición de conservación del flujo de corriente

$$
R_{11}+T_{12}=N_{1}
$$

podemos determinar la conductancia como la razón entre $I_{1}$ y $\left(\mu_{1}-\mu_{2}\right) / e$, cuyo resultado da origen a lo que se conoce como la conductancia de Landauer,

$$
G=\frac{e^{2}}{h} T \quad \text { donde } \quad T \equiv T_{12}=\sum_{m=1}^{N_{1}} \sum_{n=N_{1}+1}^{N}\left|S_{m n}\right|^{2},
$$

siendo $N$ el número total de canales, $N=N_{1}+N_{2}$; $T$ es el coeficiente de transmisión total de una guía a la otra que se conoce como conductancia adimensional.

\footnotetext{
${ }^{1}$ Aquí, las sumatorias de $T_{i j}$ y $R_{i i}, \sum_{m \in i}$ y $\sum_{n \in i}$, representan las sumas sobre los canales $m(n)$ en la guía $i(j)$.
} 


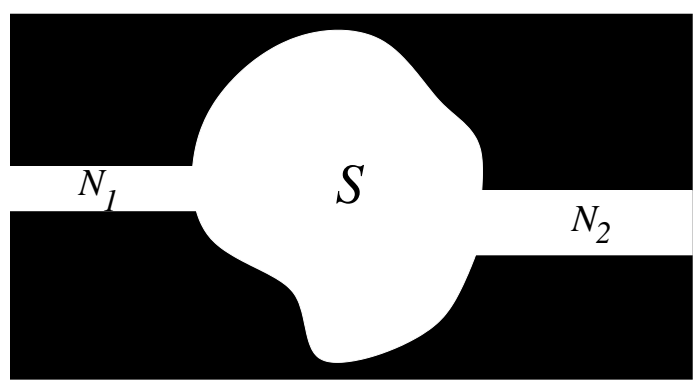

Figura 4.2: Sistema de dos terminales (guías de ondas).

\subsubsection{Fluctuaciones en un punto cuántico caótico}

Cuando el sistema es totalmente limpio pero su geometría es tal que tiene una dinámica caótica en el límite de la óptica geométrica, la conductancia fluctúa con respecto a parámetros externos. Un ejemplo se muestra en la figura 4.3 donde se grafica la conductancia adimensional $T$ como función de la energía de Fermi en unidades de $\hbar^{2} / 2 m W^{2}$, donde $m$ es la masa de la partícula y $W$ el ancho de las guías. El coeficiente de transmisión se obtuvo a través de una simulación numérica usando el formalismo de Heidelberg de la sección 2.3.2.

Las fluctuaciones de la conductancia resultan ser de carácter universal; es decir, dependen sólo de las simetrías presentes en el sistema. Para el caso de la figura 4.3 la ausencia de un campo magnético externo hace que el sistema tenga invariancia ante inversión en el tiempo. Desde el punto de vista teórico, se ha mostrado que la teoría de matrices aleatorias da una buena descripción de la estadística de las fluctuaciones de la conductancia. La distribución de $T$ para el caso de un sólo modo en cada guía, $N_{1}=N_{2}=1$, es $[25,48]$

$$
P(T)=\frac{1}{2 \sqrt{T}}
$$

mientras que para el caso de $N_{1}=N_{2}=2$ es

$$
P(T)=\left\{\begin{array}{ll}
\frac{3}{2} T & \text { para } 0<T<1, \\
\frac{3}{2}(T-2 \sqrt{T-1}) & \text { para } 1<T<2
\end{array} .\right.
$$

Estos resultados teóricos también se muestran en la figura 4.3 donde se observa que concuerdan muy bien con los resultados de la simulación numérica. 

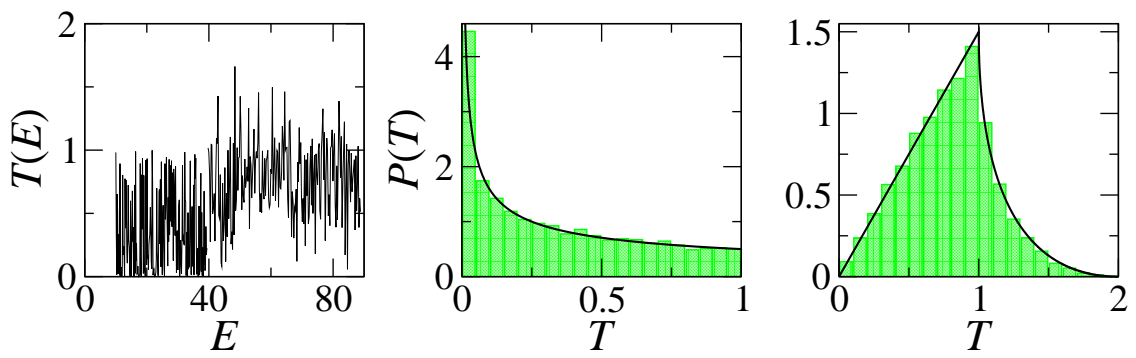

Figura 4.3: En el panel de la izquierda se muestran las fluctuaciones de la conductancia adimensional como función de la energía de Fermi $E$, en unidades de $\hbar^{2} / 2 m W^{2}$, en ausencia de campo magnético externo. Puede observase que en el intervalo de 10 a 40 sólo un canal está abierto en cada guía $(N=2)$ y de 40 a 80 están abiertos dos canales $(N=4)$. En los dos paneles de la derecha se muestran las correspondientes distribuciones comparadas con el resultado del modelo teórico de matrices aleatorias, ecuaciones (4.6) y (4.7).

\subsubsection{Analogía elástica}

Debido a que la conductancia de un sistema electrónico es proporcional al coeficiente de transmisión, la transmitancia en sistemas ondulatorios clásicos juegan el papel análogo de la conductancia adimensional.

Un experimento análogo se realizó midiendo la transmisión de ondas flexionales a través de una cavidad elástica bidimensional, en el rango acústico de frecuencias ${ }^{2}$. La forma elegida de la cavidad fue la de un medio de billar de Sinai ${ }^{3}$ conectada a dos guías de ondas, como se muestra en la figura 4.4 (figura interna en la gráfica de enmedio). La cavidad presenta invariancia ante inversión en el tiempo y la ausencia de simetría espacial tiene el propósito de anular los procesos directos de transmisión y reflexión.

Además, de acuerdo con los resultados del capítulo 3 la absorción no juega un papel relevante con la técnica usada en los sistemas elásticos. Los resultados de las mediciones muestran que la transmitancia fluctúa como función de la frecuencia, por lo que es necesario un estudio estadístico. Se espera que la estadística de las fluctuaciones sea universal, de acuerdo con la sección anterior. El autor de la presente Tesis participó en el análisis estadístico de los resultados experimentales y su interpretación física. El

\footnotetext{
${ }^{2}$ El diseño y construcción del sistema experimental, así como la medición y comportamiento de la transmitancia e interpretación de los modos abiertos en las guías de onda, constituyó parte de la tesis doctoral de Enrique Flores Olmedo [49].

${ }^{3}$ Se ha demostrado que esta geometría presenta una dinámica caótica en el límite de la óptica de rayos.
} 

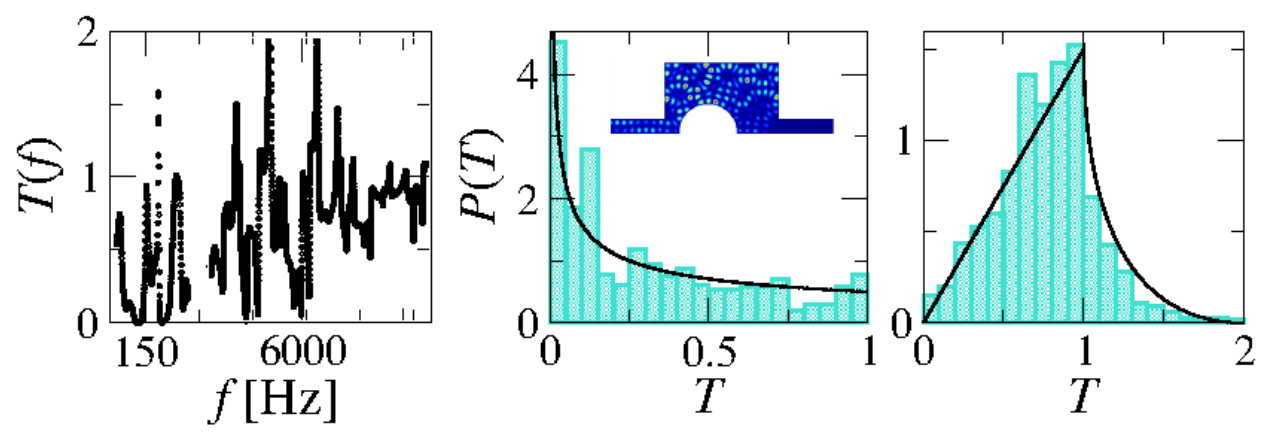

Figura 4.4: La figura de la izquierda muestra el espectro de la transmitancia $T(f)$ de ondas flexionales en dos rangos de frecuencia, que corresponde a los números de modos $N=1$ y $N=2$, respectivamente. Las gráficas de enmedio y de la derecha corresponden a las distribuciones experimentales (histogramas) y teóricas (línea continua) para la transmitancia en los dos rangos de frecuencia. La figura pequeña en la gráfica de en enmedio simula la cavidad usada en el experimento.

resultado de este análisis se muestra en la figura 4.4 para la transmitancia $T(f)$ en dos rangos de frecuencia $f$, donde se muestra el espectro en ambos intervalos de frecuencia. El primer modo, $N=1$, se encuentra en el rango de $110 \mathrm{~Hz}$ a $210 \mathrm{~Hz}$, mientras que de $5540 \mathrm{~Hz}$ a $6620 \mathrm{~Hz}$ se encuentran abiertos dos modos $N=2$. En la misma figura también se muestra la comparación de las predicciones teóricas de la sección 4.2.2, ecuaciones (4.6) y (4.7) para $N=1$ y $N=2$, respectivamente.

Estos resultados experimentales muestran que tanto la temperatura como la absorción no tienen un efecto relevante en sistemas elásticos. Por tanto, los sistemas elásticos resultan ser mejores candidatos para verificar las predicciones de la teoría de matrices aleatorias para el transporte de ondas en comparación con otro tipo de ondas [24, 50]. A pesar de la absorción y la temperatura, los sistemas elásticos mantienen la coherencia de fase [51].

\subsection{Caída de voltaje en un alambre cuántico}

Además de la conductancia, la caída de voltaje a lo largo de un alambre es otra propiedad física que ha sido de interés en el estudio del transporte electrónico. En esta sección estudiamos esta cantidad pero a lo largo de un conductor balístico, que en cada tramo de medición forma una cavidad caótica; en particular nos interesan las propiedades universales de la caída de 


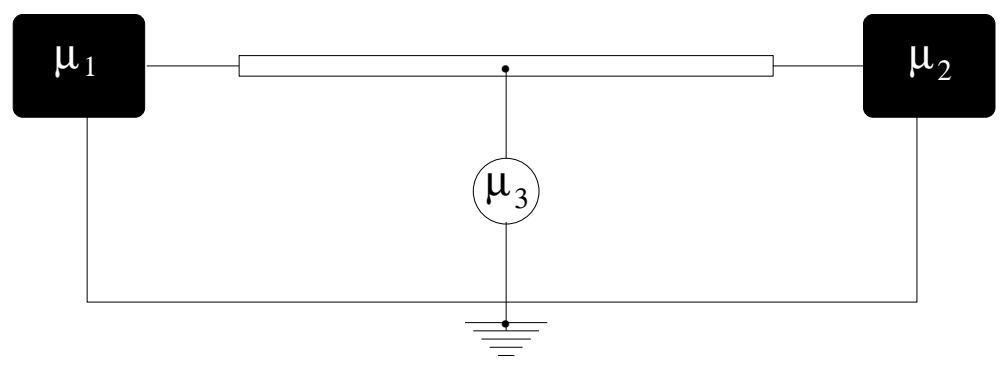

Figura 4.5: Dispositivo electrónico de tres puertos. El alambre horizontal lleva una corriente mientras que el alambre vertical mide la caída de voltaje. Las líneas delgadas representan conductores perfectos que conectan las guías de onda con los reservorios electrónicos con potenciales químicos $\mu_{1}, \mu_{2}$ y $\mu_{3}$.

voltaje. Para este propósito, construimos un dispositivo de tres terminales en el cual dos de ellas se usan para establecer una corriente y la tercera como punta o sonda de medición del voltaje. Consideraremos los casos tanto en presencia como en ausencia de invariancia ante inversión temporal.

\subsubsection{Dispositivo electrónico de tres terminales}

El sistema que nos interesa estudiar se muestra en la figura 4.5. Éste consiste de un alambre horizontal, conectado a dos reservorios puestos a potenciales $\mu_{1} \mathrm{y} \mu_{2}$, en el cual se establece un gradiente de potencial químico que da lugar a una corriente en el alambre. La punta de prueba que se usa como voltímetro mide el potencial químico $\mu_{3}$ necesario para que la corriente en ella sea nula. Los potenciales químicos están dados con respecto una referencia. En la figura 4.5 las líneas delgadas representan conductores perfectos.

De acuerdo con la ecuación (4.1), la corriente en la sonda que etiquetamos con 3 , es

$$
I_{3}=\frac{e}{h}\left[\left(N_{3}-R_{33}\right) \mu_{3}-T_{31} \mu_{1}+T_{32} \mu_{2}\right] .
$$

Igualando a cero esta ecuación y usando la condición de conservación de flujo,

$$
R_{33}+T_{31}+T_{32}=N_{3},
$$

se obtiene el potencial químico de la sonda, el cual está dado por

$$
\mu_{3}=\frac{1}{2}\left(\mu_{1}+\mu_{2}\right)+\frac{1}{2}\left(\mu_{1}-\mu_{2}\right) f,
$$




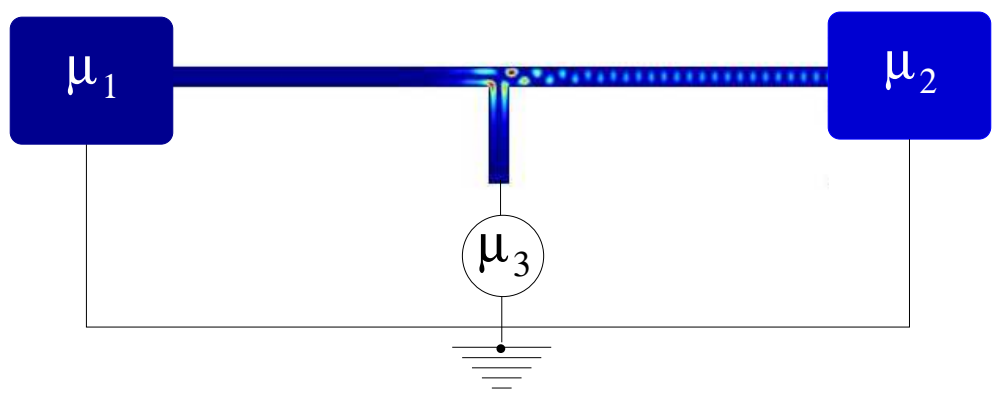

Figura 4.6: Conductor ideal, de longitud $L$ cuyo ancho soporta $N_{1}$ canales, conectado a dos potenciales químicos $\mu_{1}$ y $\mu_{2}$ por el que fluye una corriente. El alambre vertical con $N_{1}$ canales está acoplado simétricamente al alambre horizontal y mide la caída de voltaje.

donde $f$

$$
f=\frac{T_{31}-T_{32}}{T_{31}+T_{32}}
$$

con $T_{31}$ y $T_{32}$ dadas por la ecuación (4.2). La ecuación (4.10) nos dice que el potencial químico de la sonda varía alrededor del promedio entre los potenciales químicos que inducen la corriente, lo que significa que $f$ toma valores en el intervalo $[-1,1]$. Esta cantidad depende de la naturaleza específica del sistema y de las simetrías presentes.

\subsubsection{Voltaje en un conductor ideal}

En la actualidad el avance en las técnicas experimentales ha permitido la fabricación de alambres cuánticos cuyo tamaño, $L$, es mucho menor que el camino libre medio $\ell$, de manera que al no haber impurezas en el interior de los alambres la dispersión que sufren los electrones es sólo la debida a las paredes del alambre mismo. Así, supongamos que el alambre horizontal es un conductor perfecto de sección transversal constante, como se muestra en la figura 4.6. También, supongamos que la sonda de medición tiene el mismo número de canales que el alambre; es decir, $N_{3}=N_{2}=N_{1}$.

Además del confinamiento transversal, la única dispersión ocurre en la unión del alambre con la sonda (nodo) que se describe por una matriz de dispersión $S_{0}$ de dimensión $3 N_{1}$. Finalmente, si suponemos que la sonda está acoplada simétricamente al alambre, las probabilidades de transmisión entre los distintos canales a través del nodo satisfacen que

$$
T_{31}^{0}=T_{32}^{0} \text {. }
$$



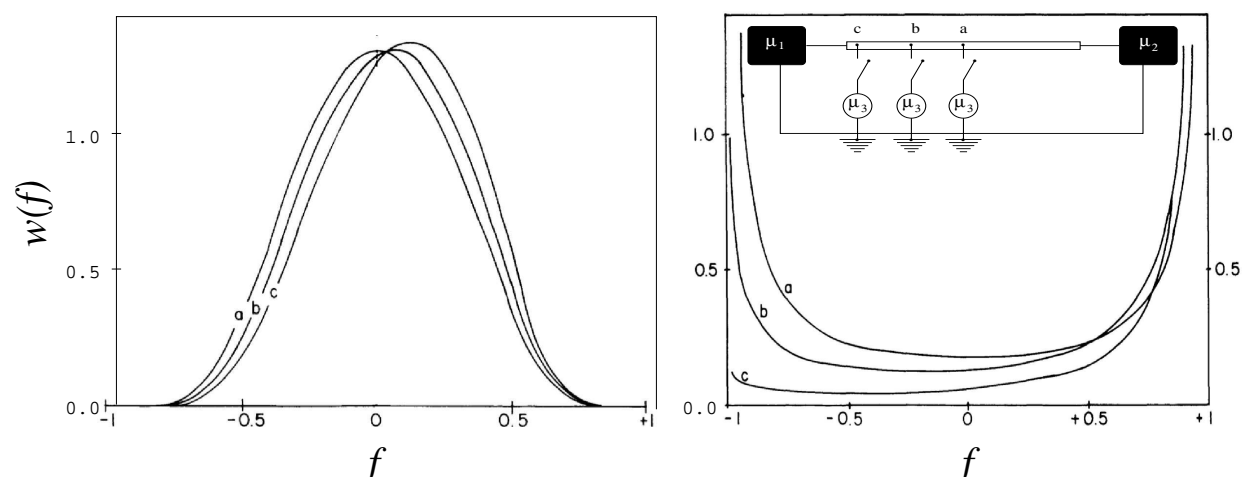

Figura 4.7: Caída de voltaje a lo largo de un alambre desordenado en el régimen balístico para $s=0.2$ (izquierda) y en el localizado para $s=20$ (derecha). Se observa una ligera dependencia en la posición de medición. En el interior de la figura de la derecha se muestran las posiciones de la sonda. Figuras modificadas de las originales en la referencia [52].

Esto significa que $f=0$, por lo que el voltaje medido a lo largo de un alambre perfecto es constante y corresponde a la media aritmética de los voltajes en ambos extremos,

$$
\mu_{3}=\frac{1}{2}\left(\mu_{1}+\mu_{2}\right) .
$$

Este resultado es interesante debido a que explica el primer término del lado derecho de la ecuación (4.10) para el caso general del dispositivo de tres terminales. Cualquier otra situación significa una desviación con respecto al alambre ideal.

\subsubsection{Fluctuaciones del voltaje en un alambre desordenado}

En el caso en que el alambre contiene impurezas en su constitución microscópica la caída de voltaje en un punto sobre él fluctúa alrededor de la media a través de $f$. El análisis de este problema en presencia de reversibilidad temporal se realizó en la referencia [52], en el caso de que el alambre soporta un sólo canal. En la figura 4.7 se muestra la distribución de $f$ en dos regímenes de la intensidad del desorden. En el límite balístico, donde la longitud $L$ de alambre es mucho menor que el camino libre medio inelástico $\ell$, la distribución tiene la forma de campana; es simétrica y con máximo en $f=0$ cuando la sonda mide el voltaje en el punto medio del alambre. Cuando la posición de medición se aleja del punto medio la forma de la distribución no 


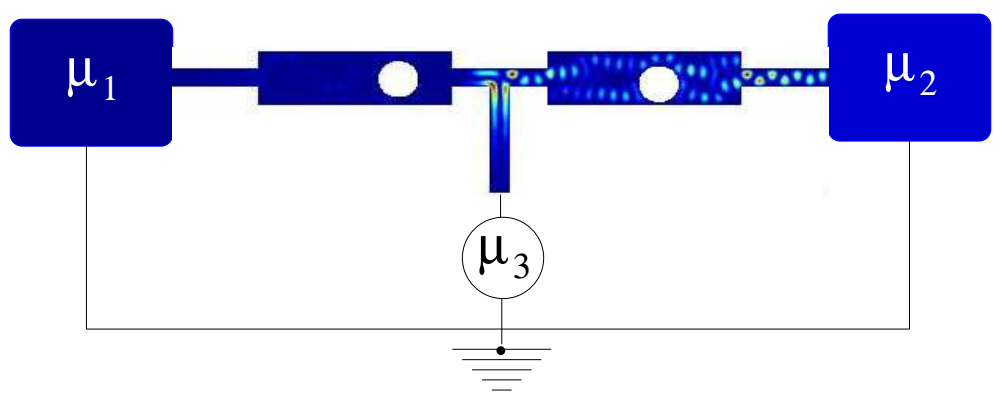

Figura 4.8: Dispositivo electrónico de tres puertos. El alambre horizontal lleva una corriente mientras que el alambre vertical mide la caída de voltaje. Las líneas delgadas representan conductores perfectos que conectan las guías de onda con los reservorios electrónicos con potenciales químicos $\mu_{1}, \mu_{2} \mathrm{y} \mu_{3}$.

cambia mucho pero se desplaza un poco del origen. La expresión analítica es [52]

$$
w(f)=\frac{1}{1-f} \frac{1}{\sqrt{\pi s\left(1-f^{2}\right)}} \mathrm{e}^{-f^{2} / s\left(1-f^{2}\right)},
$$

donde $s=L / \ell$. Este resultado vale para $s \ll 1$, de hecho $w(0)=1 / \sqrt{\pi s}$ de manera tal que

$$
\lim _{s \rightarrow 0^{+}} w(f)=\left\{\begin{array}{ll}
0 & \text { si } 0<|f|<1 \\
+\infty & \text { si } f=0
\end{array},\right.
$$

de manera que se satisface la ecuación (4.13).

En el régimen localizado, $s \gg 1$, la distribución de $f$ es cóncava y tiene un mínimo en $f=0$; también es simétrica cuando la sonda mide en el punto medio del alambre, pero lejos de éste la forma cambia y se vuelve asimétrica (figura 4.7 a la derecha).

\subsubsection{Fluctuaciones del voltaje en un alambre caótico}

Aún en alambres sin impurezas un caso de mucho interés es aquel en el que la geometría juega un papel relevante [53]. Cuando el alambre sobre el cual medimos la caída de voltaje tiene una geometría tal que induce una dinámica caótica de los electrones en el límite de la óptica de rayos, la caída de voltaje también fluctúa.

Consideremos el sistema que se muestra en la figura 4.8 en el que el alambre horizontal consta de dos cavidades caóticas conectadas por medio 

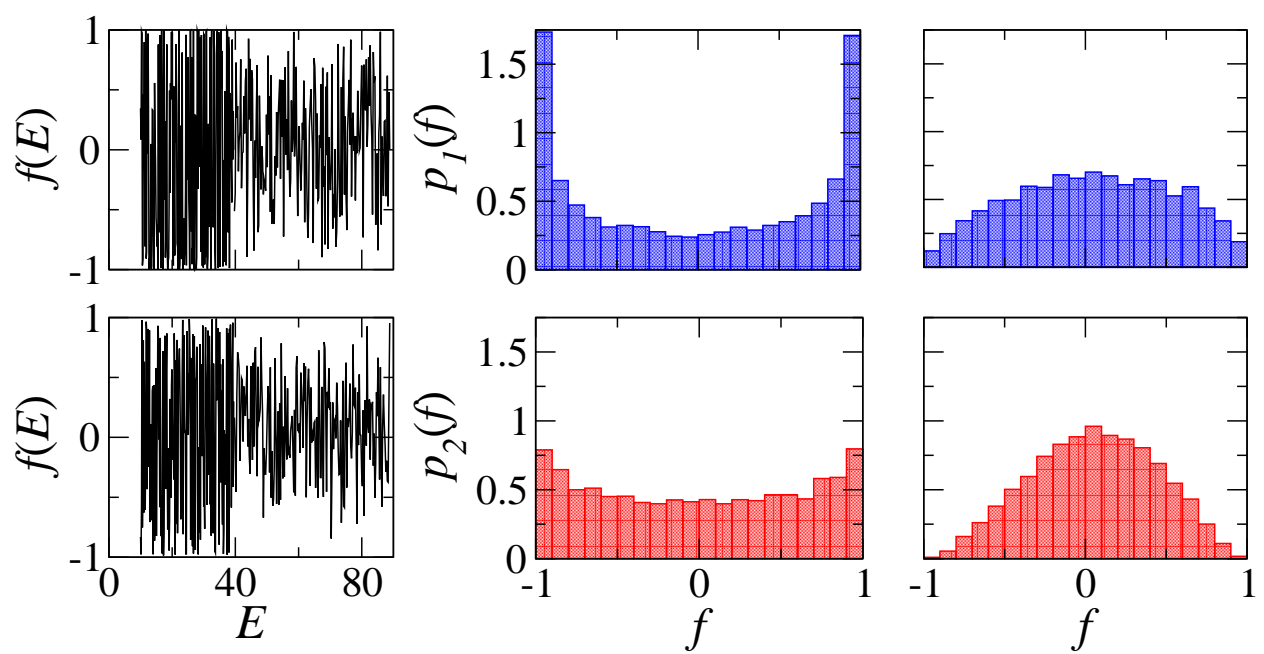

Figura 4.9: Fluctuaciones de $f$ como función de $E$ para un dispositivo electrónico de tres puertos, para las simetrías $\beta=1(2)$ en los paneles superiores (paneles inferiores). La medición se realiza por medio de la tercera terminal en los casos cuando cada una de las guías soporta $N=1$ y 2 modos de propagación, paneles superior e inferior de la derecha, respectivamente.

de conductores perfectos (terminales) a los reservorios electrónicos fijos, $\mu_{1}$ y $\mu_{2}$. En la figura 4.9 se muestra el resultado de una simulación usando el formalismo de Heidelberg de la sección 2.3.2 para el caso de uno y dos canales, tanto en presencia como en ausencia de simetría de reversibilidad temporal; es decir, para $\beta=1$ (parte superior) y $\beta=2$ (parte inferior), respectivamente. En todos los casos la distribución de $f$ es simétrica alrededor de $f=0$. Dicha distribución en el caso $\beta=1$ con un canal corresponde con el caso del régimen localizado del alambre desordenado cuando la medición se realiza en el punto medio. Esto es de esperar puesto el caso caótico puede considerarse como de máximo desorden pese a ser un sistema balístico. El resultado para $\beta=2$ no ha sido reportado. Aunque en la figura 4.9 también se muestra el resultado para dos canales, estos son sólo ilustrativos para observar la apertura de los canales. En lo que resta de esta sección proponemos un modelo estadístico que explica estos resultados en el caso de un canal.

Supongamos que cada una de las terminales soporta un modo de propagación. Los coeficientes de transmisión $T_{31}$ y $T_{32}$ de la ecuación (4.11) los podemos determinar de la matriz de dispersión total del sistema, la cual se 
puede escribir en la forma [54]

$$
S=S_{P P}+S_{P Q} S_{0} \frac{1}{1-S_{Q Q} S_{0}} S_{Q P}
$$

donde $S_{0}$ es la matriz de dispersión que describe el nodo y

$$
\begin{array}{ll}
S_{P P}=\left(\begin{array}{ccc}
r_{1} & 0 & 0 \\
0 & r_{2}^{\prime} & 0 \\
0 & 0 & 0
\end{array}\right), S_{P Q}=\left(\begin{array}{ccc}
t_{1}^{\prime} & 0 & 0 \\
0 & t_{2} & 0 \\
0 & 0 & 1
\end{array}\right), \\
S_{Q P}=\left(\begin{array}{ccc}
t_{1} & 0 & 0 \\
0 & t_{2}^{\prime} & 0 \\
0 & 0 & 1
\end{array}\right), \quad S_{Q Q}=\left(\begin{array}{ccc}
r_{1}^{\prime} & 0 & 0 \\
0 & r_{2} & 0 \\
0 & 0 & 0
\end{array}\right),
\end{array}
$$

Estas matrices están dadas en términos de los elementos de las matrices de dispersión que describen cada uno de los alambres,

$$
S_{j}=\left(\begin{array}{cc}
r_{j} & t_{j}^{\prime} \\
t_{j} & r_{j}^{\prime}
\end{array}\right), \quad j=1,2
$$

donde $r_{j}\left(r_{j}^{\prime}\right)$ y $t_{j}\left(t_{j}^{\prime}\right)$ son las amplitudes de reflexión y transmisión con incidencia desde la izquierda (derecha).

La ecuación (4.16) se puede interpretar de la siguiente manera: el primer término de la parte derecha, $S_{P P}$, representa la parte de las ondas que se reflejan cuando éstas alcanzan el conductor. El segundo término representa la dispersión múltiple que sufren las ondas al interior del dispositivo: $S_{Q P}$ da la amplitud de probabilidad de que la onda se transmita desde el exterior al interior del sistema, $S_{P Q}$ es la amplitud de probabilidad de transmisión del interior al exterior, mientras que $S_{Q Q}$ representa las reflexiones internas.

Hasta aquí, la ecuación (4.16) es un resultado general. Notemos que $S$ es una matriz unitaria $\left(S S^{\dagger}=\mathbf{1}\right)$ una vez que escogemos que $S_{0}$ también lo sea $\left(S_{0} S_{0}^{\dagger}=\mathbf{1}\right)$. Las condiciones de simetría se fijan a través de la estructura de las $S_{j}$ 's de cada una de las cavidades, y supondremos que $S_{0}$ acopla los tres alambres de manera simétrica. Esta suposición es razonable en la medida de no interferir con el proceso de medición a través de la forma en la cual se realiza dicho proceso. En ese caso, $f$ resulta ser independiente de esta matriz de acoplamiento,

$$
f=\frac{\left|t_{1}\right|^{2}\left|1+r_{2}\right|^{2}-\left|t_{2}\right|^{2}\left|1+r_{1}^{\prime}\right|^{2}}{\left|t_{1}\right|^{2}\left|1+r_{2}\right|^{2}+\left|t_{2}\right|^{2}\left|1+r_{1}^{\prime}\right|^{2}} .
$$


Es conveniente escribir las matrices $S_{j}, j=1,2$, en forma paramétrica. En la representación polar cada una de éstas toma la forma general para $\beta=2[55]$

$$
S_{j}=\left[\begin{array}{cc}
-\sqrt{1-\tau_{j}} \mathrm{e}^{\mathrm{i}\left(\phi_{j}+\phi_{j}^{\prime}\right)} & \sqrt{\tau_{j}} \mathrm{e}^{\mathrm{i}\left(\phi_{j}+\psi_{j}^{\prime}\right)} \\
\sqrt{\tau_{j}} \mathrm{e}^{\mathrm{i}\left(\phi_{j}^{\prime}+\psi_{j}\right)} & \sqrt{1-\tau_{j}} \mathrm{e}^{\mathrm{i}\left(\psi_{j}+\psi_{j}^{\prime}\right)}
\end{array}\right],
$$

con $\phi_{j}^{\prime}=\phi_{j}$ y $\psi_{j}^{\prime}=\psi_{j}$ para $\beta=1$. Con esta parametrización $f$ se escribe como

$$
f=f_{0}\left(S_{1}, S_{2}\right)
$$

donde

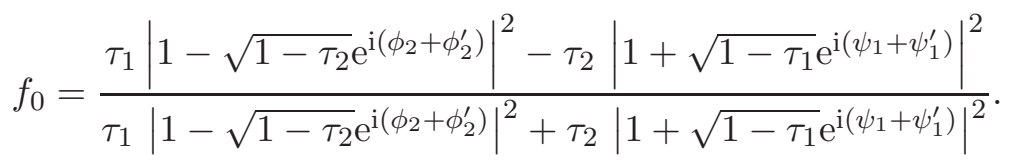

En los ensembles circulares, $\tau_{j}$ es un número aleatorio en el intervalo $[0,1]$, mientras que $\phi_{j}, \psi_{j}, \phi_{j}^{\prime}$ y $\psi_{j}^{\prime}$ son números aleatorios en el intervalo $[0,2 \pi]$, distribuidos de manera uniforme. La distribución conjunta de $S_{j}$ para ambas clases de simetría está dada por [55]

$$
d P_{\beta}\left(S_{j}\right)=2^{\beta-2} \tau_{j}^{(\beta-2) / 2} \mathrm{~d} \tau_{j} \frac{\mathrm{d} \phi_{j}}{2 \pi} \frac{\mathrm{d} \psi_{j}}{2 \pi}\left(\frac{\mathrm{d} \phi_{j}^{\prime}}{2 \pi} \frac{\mathrm{d} \psi_{j}^{\prime}}{2 \pi}\right)^{\beta-1} .
$$

La distribución para la función $f$ se obtiene de la siguiente definición

$$
p_{\beta}(f)=\int \delta\left[f-f_{0}\left(S_{1}, S_{2}\right)\right] \mathrm{d} P_{\beta}\left(S_{1}\right) \mathrm{d} P_{\beta}\left(S_{2}\right) .
$$

\section{Medición a la derecha de un alambre caótico}

Consideremos la situación particular en la cual uno de los alambres es un alambre perfecto, por ejemplo el que se encuentra del lado derecho de la sonda. En ese caso $\tau_{2}=1$ y la expresión para la distribución de $f$ se reduce a

$p_{\beta}(f)=\int \delta\left[f-\frac{\tau_{1}}{1+\sqrt{1-\tau_{1}} \cos \left(\psi_{1}+\psi_{1}^{\prime}\right)}+1\right] \frac{\mathrm{d} \tau_{1}}{\sqrt{\left(4 \tau_{1}\right)^{2-\beta}}} \frac{\mathrm{d} \psi_{1}}{2 \pi}\left(\frac{\mathrm{d} \psi_{1}^{\prime}}{2 \pi}\right)^{\beta-1}$.

El resultado de la integración se puede escribir como

$$
p_{\beta}(f)=\frac{1}{\beta \pi^{(2-\beta)}} \frac{(1-f)^{\beta / 2}}{(1+f)^{1-\beta / 2}}
$$



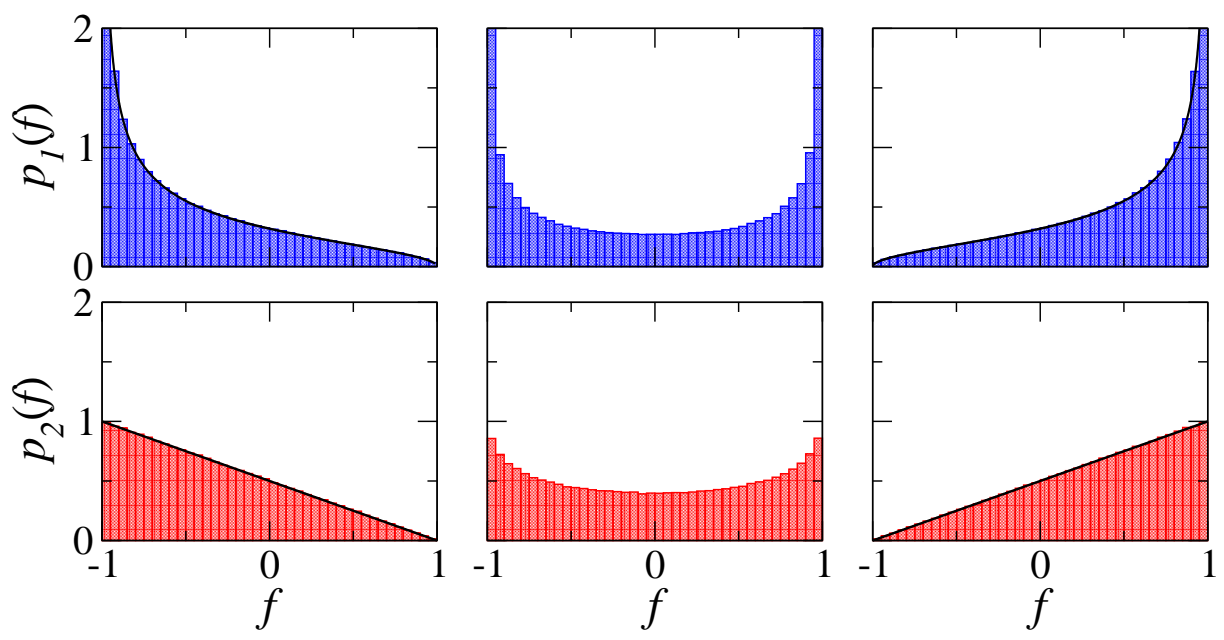

Figura 4.10: Distribución estadística de $f$ sobre un ensemble de $10^{6}$ realizaciones para las simetrías $\beta=1$ (azul) y $\beta=2$ (rojo). Las gráficas a la izquierda (derecha) se refieren a la configuración cuando el alambre 2 (1) es perfecto. En línea continua se muestran las predicciones teóricas de la ecuación (4.27). Las gráficas de enmedio muestran la distribución cuando la medición se realiza entre dos cavidades caóticas.

Cuando la medición se hace a la izquierda del alambre caótico el resultado es equivalente a cambiar $f$ por $-f$, pues la configuración es la imagen especular con respecto a la sonda de medición.

En la figura 4.10 se compara el resultado teórico de la ecuación (4.27), en línea continua con los histogramas obtenidos de la simulación numérica, para las diferentes clases de simetría $\beta=1$ y $\beta=2$, en las dos configuraciones. Además se muestra el resultado de la medición en un punto entre dos secciones del alambre, formado por dos cavidades caóticas. Estos resultados están de acuerdo con la intuición física, ya que en las figuras de la izquierda (derecha) $f$ tiende a ser predominantemente negativa (positiva), lo que implica que el potencial $\mu_{3}$ medido es muy cercano al potencial $\mu_{2}$ $\left(\mu_{1}\right)$, correspondiente al reservorio más cercano a la sonda. En cambio la medición entre los dos alambres da una distribución simétrica para $f$, lo que implica que $\mu_{3}$ fluctúa alrededor de la media aritmética de $\mu_{1}$ y $\mu_{2}$. 


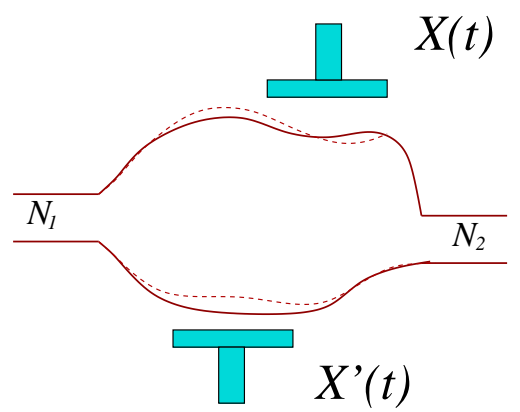

Figura 4.11: Punto cuántico conectado a dos reservorios electrónicos por medio de contactos puntuales, cada uno con $N_{1}$ y $N_{2}$ modos de propagación, respectivamente. $X(t)$ y $X^{\prime}(t)$ representan dos parámetros que modifican la forma del punto cuántico.

\subsection{Bombeo de corriente en un punto cuántico}

En la sección anterior vimos que cuando la geometría de la región de confinamiento de los electrones es tal que induce una dinámica caótica de los electrones en el límite de la óptica clásica de rayos, las propiedades de transporte muestran fluctuaciones de muestra a muestra que son de carácter universal; es decir, que sólo dependen de las simetrías presentes en el sistema. Además de la conductancia y el voltaje, otra propiedad de transporte que muestra estas fluctuaciones es la corriente bombeada a través de un punto cuántico. Una corriente CD (corriente directa) se puede establecer aún en ausencia de una diferencia de potencial, o voltaje aplicado, como resultado de la variación armónica de al menos dos parámetros externos, como aquellos que deforman la región de confinamiento, la energía de Fermi de los electrones, o un campo magnético transversal aplicado. Así, en esta sección mostramos el efecto de las simetrías presentes en el sistema sobre la corriente bombeada en un punto cuántico.

\subsubsection{Bombeo cuántico paramétrico}

Un mecanismo de bombeo de corriente se produce como resultado de perturbaciones periódicas adiabáticas en la forma del punto cuántico. La aplicación de dos voltajes AC (corriente alterna) $X(t)$ y $X^{\prime}(t)$ con una diferencia de fase entre ellas, y con una variación lenta comparada con los tiempos de relajación energéticos del sistema, producen un corriente CD total bombeada [10]. En la figura 4.11 se muestra de manera esquemática este 
mecanismo de bombeo, en donde los electrones entran a la cavidad por medio de las guías, o contactos puntuales, los cuales soportan $N_{1}$ y $N_{2}$ modos de propagación, respectivamente.

Los voltajes de perturbación que deforman la región de confinamiento de los electrones se pueden parametrizar como

$$
\begin{aligned}
X(t) & =A \cos \left(\omega t+\phi_{1}\right), \\
X^{\prime}(t) & =A^{\prime} \cos \left(\omega t+\phi_{2}\right),
\end{aligned}
$$

donde $\omega$ es la frecuencia angular, $\phi_{1}$ y $\phi_{2}$ son las fases. correspondientes de los voltajes y $A$ y $A^{\prime}$ son sus respectivas amplitudes. En el régimen adiabático, la frecuencia es mucho menor que la probabilidad de escape del electrón: $\omega \ll \Gamma / \hbar=N \Delta / 2 \pi \hbar$, con $\Delta$ el espaciamiento medio de niveles en la cavidad cuando el punto cuántico está aislado y $N=N_{1}+N_{2}$, el número total de canales. La corriente bombeada en el $m$-ésimo contacto $(m=1,2)$ está dada por $[56]$

$$
I_{m}(\varepsilon)=\frac{e \omega}{2 \pi} \int_{\mathcal{A}} \Pi_{m}\left(\varepsilon, x, x^{\prime}\right) \mathrm{d} x \mathrm{~d} x^{\prime},
$$

donde $\varepsilon=\tau_{H} \mathcal{E} / \hbar$ con $\mathcal{E}$ la energía de Fermi; $x=X / X_{c}$ y $x^{\prime}=X^{\prime} / X_{c}$ con $X_{c}$ una escala típica para los parámetros externos $X$ y $X^{\prime}$ necesaria para cambiar las propiedades de dispersión de la muestra. Aquí, la función de respuesta $\Pi_{m}\left(\varepsilon, x, x^{\prime}\right)$ está dada en términos de los elementos de la matriz de dispersión $S$ que describe al punto cuántico; es decir,

$$
\Pi_{m}\left(\varepsilon, x, x^{\prime}\right)=\frac{1}{\pi} \sum_{b} \sum_{a \in m} \operatorname{Im}\left(\frac{\partial S_{a b}^{*}}{\partial x} \frac{\partial S_{a b}}{\partial x^{\prime}}\right) .
$$

En esta ecuación el índice $a$ corre de 1 a $N_{1}$ para el contacto de la izquierda $(m=1)$ y de $N+1$ a $2 N$ para el contacto de la derecha $(m=2) ; b$ corre sobre todos los canales de la izquierda y de la derecha.

Si la amplitud de los parámetros $X$ y $X^{\prime}$ son pequeñas comparadas con $X_{c}$, entonces a primer orden $\Pi_{m}$ se vuelve independiente de $x$ y $x^{\prime}$ y la corriente bombeada $I_{m}$ se reduce a [56]

$$
I_{m}(\varepsilon)=\frac{e \omega}{2 \pi} A A^{\prime} \sin \phi \Pi_{m}(\varepsilon),
$$

donde $\phi=\phi_{1}-\phi_{2}$. Esta ecuación explica una parte de los resultados experimentales de la referencia [10], muestra que $I_{m}$ es bilineal en el voltaje CA a través del producto de las amplitudes. También muestra un comportamiento senoidal con respecto a la diferencia de fase $\phi$ entre los dos voltajes CA. En el caso en que $\phi=0$ o $\pi$, la corriente es nula, mientras que si $\phi=\pi / 2$ la corriente bombeada es máxima. 


\subsubsection{Fluctuaciones de la corriente bombeada}

Al igual que la conductancia, la corriente bombeada fluctúa al variar la energía de Fermi a través de la función $\Pi_{m}(\varepsilon)$. La distribución de estas fluctuaciones es simétrica con respecto al cero de corriente, al menos para pocos canales. Esto significa que la corriente bombeada se cuantifica por en segundo momento de la distribución.

Nuestro estudio consistirá en analizar las fluctuaciones de la corriente bombeada a través de la función $\Pi_{m}$ con respecto al ensemble cuando se tienen ciertas simetrías en el sistema. De aquí en adelante nos referiremos a esta función simplemente como la corriente y la denotaremos simplemente por $\Pi$.

\subsubsection{Análisis de matrices aleatorias para la corriente}

A través de la teoría de matrices aleatorias es directo mostrar que el promedio sobre un ensemble de la corriente bombeada es cero; es decir,

$$
\langle\Pi\rangle_{\beta}=0
$$

Entonces, la corriente bombeada se cuantifica por la desviación cuadrática media,

$$
\sigma_{\beta}(\Pi)=\sqrt{\left\langle\Pi^{2}\right\rangle_{\beta}}
$$

Para determinar las fluctuaciones de $\Pi$ es conveniente parametrizar la matriz de dispersión. Siendo $S$ una matriz unitaria de dimensión $N$, una parametrización conveniente es escribirla como

$$
S=U V
$$

donde $U$ y $V$ son matrices unitarias más generales posible para $\beta=2 \mathrm{y}$ $V=U^{T}$ para $\beta=1$. Con esta parametrización, las derivada energética definida en la ecuación (2.8) se escribe como

$$
\frac{\partial S}{\partial \varepsilon}=\mathrm{i} U Q_{\varepsilon} V
$$

y de manera equivalente las derivadas paramétricas se definen como

$$
\frac{\partial S}{\partial x}=\mathrm{i} U Q_{x} V \quad \text { y } \quad \frac{\partial S}{\partial x^{\prime}}=\mathrm{i} U Q_{x^{\prime}} V
$$


donde $Q_{x, x^{\prime}}$ son matrices Hermitianas de dimensión $N$ que dependen de $Q_{\varepsilon}$ [35], de hecho la distribución conjunta de $S, Q_{\varepsilon}, Q_{x}$ y $Q_{x^{\prime}}$ está dada por [57]

$$
P \propto\left(\operatorname{det} Q_{\varepsilon}\right)^{-9 N / 2} \exp \left\{-\operatorname{tr}\left[Q_{\varepsilon}^{-1}+\frac{1}{8}\left(Q_{\varepsilon}^{-1} Q_{x}\right)^{2}+\frac{1}{8}\left(Q_{\varepsilon}^{-1} Q_{x^{\prime}}\right)^{2}\right]\right\} .
$$

Notemos que $S$ es estadísticamente independiente de $Q_{\varepsilon}, Q_{x}$ y $Q_{x^{\prime}}$ mientras que estas dos últimas matrices tienen distribución Gaussiana con media cero y anchura definida por $Q_{\varepsilon}[35]$.

La parametrización dada en las ecuaciones (4.35) y (4.37) nos permite escribir la corriente $\Pi$ en términos de las matrices $U, V, Q_{x}$ y $Q_{x^{\prime}}$. Para $\beta=1$

$$
\Pi=\frac{1}{2 \pi \mathrm{i}} \sum_{a=1}^{N_{1}} \sum_{b=1}^{N} \sum_{c, d=1}^{N} \sum_{c^{\prime}, d^{\prime}=1}^{N} U_{a c} U_{b d} U_{a c^{\prime}}^{*} U_{b d^{\prime}}^{*}\left(Q_{c d}^{x^{\prime}} Q_{c^{\prime} d^{\prime}}^{x *}-Q_{c^{\prime} d^{\prime}}^{x^{\prime} *} Q_{c d}^{x}\right),
$$

mientras que para $\beta=2$

$$
\Pi=\frac{1}{2 \pi \mathrm{i}} \sum_{a=1}^{N_{1}} \sum_{b=1}^{N} \sum_{c, d=1}^{N} \sum_{c^{\prime}, d^{\prime}=1}^{N} U_{a c} U_{a c^{\prime}}^{*} V_{d b} V_{d^{\prime} b}^{*}\left(Q_{c d}^{x^{\prime}} Q_{c^{\prime} d^{\prime}}^{x *}-Q_{c^{\prime} d^{\prime}}^{x^{\prime} *} Q_{c d}^{x}\right) .
$$

De esta manera, para $\beta=1$

$$
\begin{aligned}
\left\langle\Pi^{2}\right\rangle_{1} & =-\frac{1}{4 \pi^{2}} \sum_{a=1}^{N_{1}} \sum_{b=1}^{N} \sum_{c, d=1}^{N} \sum_{c^{\prime}, d^{\prime}=1}^{N} \sum_{\alpha=1}^{N_{1}} \sum_{\beta=1}^{N} \sum_{\gamma, \delta=1}^{N} \sum_{\gamma^{\prime}, \delta^{\prime}=1}^{N} M_{a c, b d, \alpha \gamma, \beta \delta}^{a c^{\prime}, b d^{\prime}, \alpha \gamma^{\prime}, \beta \delta^{\prime}} \\
& \times\left\langle\left(Q_{c d}^{x^{\prime}} Q_{c^{\prime} d^{\prime}}^{x *}-Q_{c^{\prime} d^{\prime}}^{x^{\prime} *} Q_{c d}^{x}\right)\left(Q_{\gamma \delta}^{x^{\prime}} Q_{\gamma^{\prime} \delta^{\prime}}^{x *}-Q_{\gamma^{\prime} \delta^{\prime}}^{x^{*} *} Q_{\gamma \delta}^{x}\right)\right\rangle
\end{aligned}
$$

$\mathrm{y} \operatorname{para} \beta=2$

$$
\begin{aligned}
\left\langle\Pi^{2}\right\rangle_{2} & =-\frac{1}{4 \pi^{2}} \sum_{a=1}^{N_{1}} \sum_{b=1}^{N} \sum_{c, d=1}^{N} \sum_{c^{\prime}, d^{\prime}=1}^{N} \sum_{\alpha=1}^{N_{1}} \sum_{\beta=1}^{N} \sum_{\gamma, \delta=1}^{N} \sum_{\gamma^{\prime}, \delta^{\prime}=1}^{N} M_{a c, \alpha \gamma}^{a c^{\prime}, \alpha \gamma^{\prime}} M_{d b, \delta \beta}^{d^{\prime} b, \delta^{\prime} \beta} \\
& \times\left\langle\left(Q_{c d}^{x^{\prime}} Q_{c^{\prime} d^{\prime}}^{x *}-Q_{c^{\prime} d^{\prime}}^{x^{\prime} *} Q_{c d}^{x}\right)\left(Q_{\gamma \delta}^{x^{\prime}} Q_{\gamma^{\prime} \delta^{\prime}}^{x *}-Q_{\gamma^{\prime} \delta^{\prime}}^{x^{\prime} *} Q_{\gamma \delta}^{x}\right)\right\rangle
\end{aligned}
$$

donde

$$
\begin{gathered}
M_{a c, b d, \alpha \gamma, \beta \delta}^{a c^{\prime}, b d^{\prime}, \alpha \gamma^{\prime}, \beta \delta^{\prime}}=\left\langle U_{a c} U_{b d} U_{\alpha \gamma} U_{\beta \delta} U_{a c^{\prime}}^{*} U_{b d^{\prime}}^{*} U_{\alpha \gamma^{\prime}}^{*} U_{\beta \delta^{\prime}}^{*}\right\rangle, \\
M_{a c, \alpha \gamma}^{a c^{\prime}, \alpha \gamma^{\prime}}=\left\langle U_{a c} U_{\alpha \gamma} U_{a c^{\prime}}^{*} U_{\alpha \gamma^{\prime}}^{*}\right\rangle \quad \text { y } \quad M_{d b, \delta \beta}^{d^{\prime} b, \delta^{\prime} \beta}=\left\langle V_{d b} V_{\delta \beta} V_{d^{\prime} b}^{*} V_{\delta^{\prime} \beta}^{*},\right\rangle
\end{gathered}
$$


son promedios sobre el grupo unitario de matrices conocidos en la literatura [58]. De acuerdo con la distribución (4.38) los promedios sobre $Q_{x} \mathrm{y}$ $Q_{x^{\prime}}$ se reducen a promedios sobre $Q_{\varepsilon}$. A través de un cálculo bastante largo, pero directo, se puede mostrar que

$$
\begin{aligned}
\left\langle\Pi^{2}\right\rangle_{\beta} & =-\frac{8}{\pi^{2}} \frac{N_{1} N_{2}}{N\left(N^{2}-1\right)}\left[\left\langle\frac{2}{\beta} \sum_{a=1}^{N}\left(Q_{\varepsilon}^{4}\right)_{a a}-\sum_{a, b, c=1}^{N}\left(Q_{\varepsilon}^{2}\right)_{a a}\left(Q_{\varepsilon}\right)_{b b}\left(Q_{\varepsilon}\right)_{c c}\right\rangle\right. \\
& \left.+(2-\beta)\left\langle\sum_{a, b=1}^{N}\left(Q_{\varepsilon}^{2}\right)_{a a}\left(Q_{\varepsilon}^{2}\right)_{b b}-2 \sum_{a, b=1}^{N}\left(Q_{\varepsilon}^{3}\right)_{a a}\left(Q_{\varepsilon}\right)_{b b}\right\rangle\right]
\end{aligned}
$$

Con un poco de álgebra, $\left\langle\Pi^{2}\right\rangle_{\beta}$ se puede escribir en términos de los primeros momentos conjuntos de los tiempos de retardo propios adimensionales. Esto es,

$$
\left\langle\Pi^{2}\right\rangle_{\beta}=\frac{8}{\pi^{2}} \frac{N_{1} N_{2}}{N+1}\left[\frac{4}{\beta}\left\langle q_{1}^{3} q_{2}\right\rangle_{\beta}+(N-2)\left\langle q_{1}^{2} q_{2} q_{3}\right\rangle_{\beta}+(\beta-1)\left\langle q_{1}^{2} q_{2}^{2}\right\rangle_{\beta}\right],
$$

donde los momentos $\left\langle q_{1}^{3} q_{2}\right\rangle,\left\langle q_{1}^{2} q_{2} q_{3}\right\rangle$ y $\left\langle q_{1}^{2} q_{2}^{2}\right\rangle$ están dados por [59]

$$
\begin{aligned}
\left\langle q_{1}^{3} q_{2}\right\rangle_{\beta} & =\frac{2\left(3 N^{2}+N+2-\frac{\beta}{2}\right)}{N(N+1)(N+2)(N+3)\left(N-\frac{2}{\beta}\right)\left(N-\frac{4}{\beta}\right)}, \\
\left\langle q_{1}^{2} q_{2} q_{3}\right\rangle_{\beta} & =\frac{2}{(N+1)(N+2)(N+3)\left(N-\frac{2}{\beta}\right)}, \\
\left\langle q_{1}^{2} q_{2}^{2}\right\rangle_{\beta} & =\frac{2(2 N+1)}{(N+1)(N+2)(N+3)\left(N-\frac{2}{\beta}\right)\left(N+1-\frac{2}{\beta}\right)},
\end{aligned}
$$

de donde finalmente obtenemos

$$
\begin{aligned}
\left\langle\Pi^{2}\right\rangle_{\beta}= & \frac{16}{\pi^{2}} \frac{N_{1} N_{2}}{N+1} \frac{1}{(N+1)(N+2)(N+3)\left(N-\frac{2}{\beta}\right)} \\
& \times\left[\frac{\beta}{4} \frac{3 N^{2}+N+2-\frac{\beta}{2}}{N(N-4)}+(\beta-1) \frac{2 N+1}{N+1-\frac{2}{\beta}}+N-2\right] .
\end{aligned}
$$




\section{Capítulo 5}

\section{Tiempos de retardo}

El estudio de las fluctuaciones estadísticas de varias propiedades de transporte a través de cavidades con dinámica clásicamente caótica, tales como puntos cuánticos y cavidades de microondas con acoplamiento ideal entre las cavidades con los $N$ canales abiertos, depende de los momentos conjuntos de los tiempos de retardo propios. Así, en este capítulo, nuestro propósito es evaluar dichos momentos para cualquier clase de simetría, $\beta=1,2$ y 4 (ortogonal, unitaria y simpléctica, respectivamente) y un número arbitrario de canales.

\subsection{Momentos generalizados de los tiempos de re- tardo propios}

Los momentos generalizados de los tiempos de retardo propios, en unidades adimensionales,

$$
\left\langle q_{1}^{k_{1}} \cdots q_{N}^{k_{N}}\right\rangle^{(\beta)}=\left\langle x_{1}^{-k_{1}} \cdots x_{N}^{-k_{N}}\right\rangle^{(\beta)}=\int_{0}^{\infty} \frac{P_{\beta}\left(x_{1}, \ldots, x_{N}\right)}{x_{1}^{k_{1}} \cdots x_{N}^{k_{N}}} \mathrm{~d} x_{1} \cdots \mathrm{d} x_{N}
$$

con $k_{j}(j=1, \ldots, N)$ un número entero, se conocen como los momentos negativos generalizados del ensemble de Laguerre. Aquí, las $q_{i}$ 's $(i=1, \ldots, N)$ son los tiempos de retardo propios medidos en unidades del tiempo de Heisenberg $\tau_{H}$; esto es, $q_{i}=\tau_{i} / \tau_{H}, x_{i}=\tau_{H} / \tau_{i}$ son los recíprocos de los tiempos de retardo propios cuya distribución está dada por el ensemble de Laguerre, ecuación (2.9).

Para obtener una expresión general manejable para los momentos con- 
juntos notemos que

$$
\prod_{a<b \leq N}\left(x_{b}-x_{a}\right)=\operatorname{det} V_{N}=\left|\begin{array}{ccccc}
1 & 1 & 1 & \cdots & 1 \\
x_{1} & x_{2} & x_{3} & \cdots & x_{N} \\
\vdots & \vdots & \vdots & \cdots & \vdots \\
x_{1}^{N-1} & x_{2}^{N-1} & x_{3}^{N-1} & \cdots & x_{N}^{N-1}
\end{array}\right|,
$$

que se conoce como determinante de Vandermonde de orden $N$ [60]. Se puede probar que [61]

$$
\operatorname{det} V_{N}=\sum_{\sigma} \operatorname{sgn} \sigma \prod_{a=1}^{N} x_{a}^{-1+\sigma(a)},
$$

donde $\sigma$ es la permutación que pertenece al grupo simétrico de grado $N$ [62],

$$
S_{N}=\{\sigma:\{1, \ldots, N\} \rightarrow\{1, \ldots, N\} \mid \sigma \text { es una permutación }\},
$$

con $\operatorname{sgn} \sigma$ el signo de $\sigma$; la permutación identidad es $\iota(a)=a$ para $a=$ $1, \ldots, N$.

Aquí es necesario introducir algunas definiciones y propiedades.

Definición 1. Para $t_{a} \geq 0(a=1, \ldots, N)$, sea

$$
\Delta_{N}=\left\{\left(t_{1}, \ldots, t_{N}\right): 0 \leq t_{1} \leq t_{2} \leq \cdots \leq t_{N}\right\} .
$$

Por lo tanto, el determinante de Vandermonde de la ecuación (5.2) se vuelve positiva para $\left(x_{1}, \ldots, x_{N}\right) \in \Delta_{N}$, tal que

$$
\operatorname{det} V_{N}=\left|\operatorname{det} V_{N}\right| \text {, }
$$

y la igualdad en la ecuación (5.3) se puede escribir como

$$
\left|\operatorname{det} V_{N}\right|^{\beta}=\sum_{\sigma_{1}, \ldots, \sigma_{\beta}} \prod_{i=1}^{\beta} \operatorname{sgn} \sigma_{i} \prod_{a=1}^{N} x_{a}^{-\beta+\sum_{j=1}^{\beta} \sigma_{j}(a)},
$$

donde $\sigma_{j} \in S_{N}(j=1, \ldots, \beta)$.

Cada permutación $\sigma \in S_{N}$ está asociada a una transformación unitaria $\pi_{\sigma}$ en el espacio Euclidiano $N$-dimensional:

$$
\left(x_{1}, \ldots, x_{N}\right) \longrightarrow \pi_{\sigma}\left(x_{1}, \ldots, x_{N}\right)=\left[x_{\sigma(1)}, \ldots, x_{\sigma(N)}\right] .
$$

Definición 2. Para cualquier $\eta \in S_{N}$ y $t_{a} \geq 0(a=1, \ldots, N)$, sea

$$
\Delta_{N}^{\eta}=\pi_{\eta}^{-1}\left(\Delta_{N}\right):=\left\{\left(t_{1}, \ldots, t_{N}\right): 0 \leq t_{\eta(1)} \leq t_{\eta(2)} \leq \cdots \leq t_{\eta(N)}\right\} .
$$


Notemos que $\Delta_{N}^{\iota}=\Delta_{N} ;$ también, para cualquier $\eta \in S_{N}$ y $\left(t_{1}, \ldots, t_{N}\right) \in$ $\Delta_{N}^{\eta}$, de la ecuación (5.8)

$$
\pi_{\eta}^{-1}\left(t_{1}, \ldots, t_{N}\right)=\left[t_{\eta^{-1}(1)}, \ldots, t_{\eta^{-1}(N)}\right] \in \Delta_{N}
$$

y por lo tanto

$$
\operatorname{det} V_{N}\left[\pi_{\eta}^{-1}\left(t_{1}, \ldots, t_{N}\right)\right]=\prod_{a<b}\left[t_{\eta^{-1}(b)}-t_{\eta^{-1}(a)}\right] \geq 0,
$$

donde hemos tomado en cuenta la ecuación (5.6). De aquí,

$$
\left|\operatorname{det} V_{N}\left(t_{1}, \ldots, t_{N}\right)\right|=\operatorname{det} V_{N}\left[\pi_{\eta}^{-1}\left(t_{1}, \ldots, t_{N}\right)\right] .
$$

Finalmente, para cualquier función medible no negativa $f\left(t_{1}, \ldots, t_{N}\right)$, el Teorema del Cambio de Variables nos permite escribir

$$
\begin{aligned}
\int_{0}^{\infty} f\left(t_{1}, \ldots, t_{N}\right) \mathrm{d} t_{1} \cdots \mathrm{d} t_{N} & =\sum_{\eta \in S_{N}} \int_{\Delta_{N}^{\eta}} f\left(t_{1}, \ldots, t_{N}\right) \mathrm{d} t_{1} \cdots \mathrm{d} t_{N} \\
& =\sum_{\eta \in S_{N}} \int_{\Delta_{N}} f \circ \pi_{\eta}^{-1}\left(t_{1}, \ldots, t_{N}\right) \mathrm{d} t_{1} \cdots \mathrm{d} t_{N} .
\end{aligned}
$$

\subsubsection{Momentos negativos de la distribución de Laguerre de $N$ variables}

De la definición del determinante de Vandermonde (5.2) y la ecuación (5.7), la distribución de Laguerre de la ecuación (2.9) se puede escribir como

$$
P_{\beta}\left(x_{1}, \ldots, x_{N}\right)=C_{N}^{(\beta)} \sum_{\sigma_{1}, \ldots, \sigma_{\beta}} \prod_{i=1}^{\beta} \operatorname{sgn} \sigma_{i} \prod_{a=1}^{N} x_{a}^{\gamma_{a}} \mathrm{e}^{-\beta x_{a} / 2},
$$

donde

$$
\gamma_{a}:=\gamma_{a}^{\sigma_{1}, \ldots, \sigma_{\beta}}=\frac{N \beta}{2}-\beta+\sum_{j=1}^{\beta} \sigma_{j}(a) \quad \text { para } \quad a=1, \ldots, N .
$$

Es conveniente escribir $P_{\beta}\left(x_{1}, \ldots, x_{N}\right)$ como

$$
P_{\beta}\left(x_{1}, \ldots, x_{N}\right)=C_{N}^{(\beta)} \sum_{\sigma_{1}, \ldots, \sigma_{\beta}} \prod_{i=1}^{\beta} \operatorname{sgn} \sigma_{i} \prod_{a=1}^{N}\left(\frac{2}{\beta}\right)^{\gamma_{a}+1} \Gamma\left(\gamma_{a}+1\right) f_{\gamma_{a}+1, \beta / 2}\left(x_{a}\right),
$$


donde $f_{u, v}(t)$ es la función densidad de probabilidad de la distribución Gamma con parámetros $u$ y $v[63,64]$,

$$
f_{u, v}(t)=\frac{v^{u} t^{u-1} \mathrm{e}^{-u t}}{\Gamma(u)}
$$

con $\Gamma(u)$ la función Gamma [64]. Es importante notar que $\Gamma\left(\gamma_{a}+1\right)$ está bien definida.

Para los momentos conjuntos en los que estamos interesados necesitamos la función auxiliar

$$
R_{\beta}\left(x_{1}, \ldots, x_{N}\right)=\frac{P_{\beta}\left(x_{1}, \ldots, x_{N}\right)}{x_{1}^{k_{1}} \cdots x_{N}^{k_{N}}},
$$

para $k_{a}(a=1, \ldots, N)$ un número entero. Usando la ecuación (5.16) esta función se puede escribir como

$$
R_{\beta}\left(x_{1}, \ldots, x_{N}\right)=C_{N}^{(\beta)} \sum_{\sigma_{1}, \ldots, \sigma_{\beta}} \prod_{i=1}^{\beta} \operatorname{sgn} \sigma_{i} \prod_{a=1}^{N}\left(\frac{2}{\beta}\right)^{\alpha_{a}} \Gamma\left(\alpha_{a}\right) f_{\alpha_{a}, \beta / 2}\left(x_{a}\right),
$$

donde $\alpha_{a}=\gamma_{a}-k_{a}+1$ para $a=1, \ldots, N$, con $\gamma_{a}$ dada en la ecuación (5.15). Por tanto, para cualquier $\eta \in S_{N}$ y $\left(x_{1}, \ldots, x_{N}\right) \in \Delta_{N}^{\eta}$, las propiedades (5.10) y (5.12) nos permite escribir

$$
R_{\beta} \circ \pi_{\eta}^{-1}\left(x_{1}, \ldots, x_{N}\right)=\frac{P_{\beta}\left(x_{1}, \ldots, x_{N}\right)}{x_{\eta^{-1}(1)}^{k_{1}} \cdots x_{\eta^{-1}(N)}^{k_{N}}}=\frac{P_{\beta}\left(x_{1}, \ldots, x_{N}\right)}{x_{1}^{k_{\eta(1)}} \cdots x_{N}^{k_{\eta(N)}}} .
$$

La última igualdad es justo la definición (5.18), la cual de acuerdo con la ecuación (5.19), se puede escribir como

$$
R_{\beta} \circ \pi_{\eta}^{-1}\left(x_{1}, \ldots, x_{N}\right)=C_{N}^{(\beta)} \sum_{\sigma_{1}, \ldots, \sigma_{\beta}} \prod_{i=1}^{\beta} \operatorname{sgn} \sigma_{i} \prod_{a=1}^{N}\left(\frac{2}{\beta}\right)^{\alpha_{a}^{\eta}} \Gamma\left(\alpha_{a}^{\eta}\right) f_{\alpha_{a}^{\eta}, \beta / 2}\left(x_{a}\right),
$$

donde

$$
\alpha_{a}^{\eta}=\gamma_{a}-k_{\eta(a)}+1, \quad \text { para } \quad a=1, \ldots, N
$$

Si integramos esta ecuación sobre $\Delta_{N}$ y sumamos sobre $\eta$, la propiedad (5.13) nos permite llegar al resultado deseado, es decir

$$
\left\langle x_{1}^{-k_{1}} \cdots x_{N}^{-k_{N}}\right\rangle^{(\beta)}=C_{N}^{(\beta)} \sum_{\eta, \sigma_{1}, \ldots, \sigma_{\beta}} F_{\theta_{\eta, N}} \prod_{i=1}^{\beta} \operatorname{sgn} \sigma_{i} \prod_{a=1}^{N}\left(\frac{2}{\beta}\right)^{\alpha_{a}^{\eta}} \Gamma\left(\alpha_{a}^{\eta}\right) .
$$


donde $F_{\theta_{\eta, N}}$ está definida como

$$
F_{\theta_{\eta, N}}=\int_{\Delta_{N}} \prod_{a=1}^{N} f_{\alpha_{a}^{\eta}, \beta / 2}\left(x_{a}\right) \mathrm{d} x_{a}
$$

con

$$
\theta_{\eta, N}:=\theta_{\eta, N}^{\sigma_{1}, \ldots, \sigma_{\beta}}:=(\alpha_{1}^{\eta}, \ldots, \alpha_{N}^{\eta}, \underbrace{\beta / 2, \ldots, \beta / 2}_{N}) .
$$

Es claro que la constante de normalización $C_{N}^{(\beta)}$ se puede obtener de la ecuación (5.23) para $k_{a}=0(a=1, \ldots, N)$, en cuyo caso el argumento en la suma de la ecuación (5.23) es independiente de $\eta$, tal que la suma sobre $\eta$ es exactamente $N$ !, y está dada por

$$
C_{N}^{(\beta)}=\left[N ! \sum_{\sigma_{1}, \ldots, \sigma_{\beta}} F_{\theta_{N}} \prod_{i=1}^{\beta} \operatorname{sgn} \sigma_{i} \prod_{a=1}^{N}\left(\frac{2}{\beta}\right)^{\gamma_{a}+1} \Gamma\left(\gamma_{a}+1\right)\right]^{-1},
$$

donde

$$
F_{\theta_{N}}=\int_{\Delta_{N}} \prod_{a=1}^{N} f_{\gamma_{a}+1, \beta / 2}\left(x_{a}\right) \mathrm{d} x_{a} .
$$

Aquí vale la pena hacer las siguientes dos observaciones.

Observación 1. En las ecuaciones (5.23) y (5.26), es necesario tomar en cuenta la dependencia de los parámetros $\theta_{N}$ y $\theta_{\eta, N}$ en las permutaciones $\sigma_{1}, \ldots, \sigma_{\beta}$, pero por simplicidad omitimos escribirlas explícitamente.

Observación 2. Ya que el mínimo valor posible de cualquier permutación es 1 , es fácil mostrar que $\gamma_{a}-k_{a}+1>0$ para $0 \leq k_{a}<\frac{N \beta}{2}+1$.

Esta última observación asegura que la función Gamma que aparece en la ecuación (5.23) está bien definida. Es importante notar que la propiedad expresada en la observación 2 es un resultado general que restringe los valores de $k_{a}(a=1, \ldots, N)$.

\subsection{2. $\beta$ número entero par}

Cuando $\beta$ es un número positivo entero par, entonces $\left|\operatorname{det} V_{N}\right|^{\beta}=\left(\operatorname{det} V_{N}\right)^{\beta}$ para cualquier $\left(x_{1}, \ldots, x_{N}\right) \in \mathbb{R}^{N}$, con $\mathbb{R}^{N}$ el espacio Euclidiano real $N$ dimensional. En este caso no es necesario usar la ecuación (5.13), así en los cálculos de los momentos negativos obtenemos fórmulas más simples para los momentos negativos conjuntos de los tiempos de retardo. 
Si integramos la ecuación (5.19) tenemos que

$$
\begin{aligned}
\left\langle x_{1}^{-k_{1}} \cdots x_{N}^{-k_{N}}\right\rangle^{(\beta)}= & C_{N}^{(\beta)}\left(\frac{2}{\beta}\right)^{N} \sum_{\sigma_{1}, \ldots, \sigma_{\beta}} \prod_{i=1}^{\beta} \operatorname{sgn} \sigma_{i} \\
& \times \prod_{a=1}^{N} \int_{0}^{\infty}\left(\frac{\beta}{2}\right) x^{\gamma_{a}-k_{a}} \mathrm{e}^{-\beta x / 2} \mathrm{~d} x \\
= & C_{N}^{(\beta)}\left(\frac{2}{\beta}\right)^{N} A_{N}^{(\beta)}\left(k_{1}, \ldots, k_{N}\right)
\end{aligned}
$$

donde

$$
A_{N}^{(\beta)}\left(k_{1}, \ldots, k_{N}\right)=\sum_{\sigma_{1}, \ldots, \sigma_{\beta}} \prod_{i=1}^{\beta} \operatorname{sgn} \sigma_{i} \prod_{a=1}^{N}\left(\frac{2}{\beta}\right)^{\gamma_{a}-k_{a}} \Gamma\left(\gamma_{a}-k_{a}+1\right)
$$

Si $k_{1}=\cdots=k_{N}=0$, entonces la ecuación (5.28) implica que $C_{N}^{(\beta)}=$ $(\beta / 2)^{N} / A_{N}^{(\beta)}, \operatorname{con} A_{N}^{(\beta)}=A_{N}^{(\beta)}(0, \ldots, 0)$.

En las tres secciones siguientes presentamos cálculos explícitos con $N=1$ y 2 , para $\beta=1$ y 4, y $N=1,2,3$ y 4 para $\beta=2$. Somos explícitos en las correspondientes permutaciones tanto como nos es posible. Aquí comenzamos nuestros cálculos con el caso $\beta=1$, ya que normalmente es el caso más difícil de tratar analíticamente.

\subsection{Cálculos explícitos para $\beta=1$}

Para el caso particular de $\beta=1$ con $N$ arbitrario, la ecuación (5.15) la escribimos como

$$
\gamma_{a}:=\gamma_{a}^{\sigma_{1}}=\frac{N}{2}-1+\sigma_{1}(a), \quad \text { para } \quad a=1, \ldots, N,
$$

tal que los momentos dados por las ecuaciones (5.23) se simplifican como

$$
\begin{aligned}
\left\langle x_{1}^{-k_{1}} \cdots x_{N}^{-k_{N}}\right\rangle^{(1)}= & C_{N}^{(1)} \sum_{\eta, \sigma_{1}} F_{\theta_{\eta, N}} \operatorname{sgn} \sigma_{1} \prod_{a=1}^{N} 2^{\sigma_{1}(a)-k_{\eta(a)}+N / 2} \\
& \times \Gamma\left[\sigma_{1}(a)-k_{\eta(a)}+N / 2\right]
\end{aligned}
$$

donde $0 \leq k_{a}<1+N / 2$. 


\subsubsection{Caso $N=1$}

De acuerdo a la ecuación (5.4), $S_{1}=\{\iota(1)=1\}$, esto es, solo la identidad pertenece al grupo simétrico $S_{1}$, tal que $\eta(1)=\sigma_{1}(1)=\iota(1)=1$ y $\theta_{\eta, 1}=$ $\theta_{1}=(3 / 2-k, 1 / 2)$. Por tanto, de la ecuación (5.31),

$$
\begin{aligned}
\left\langle x^{-k}\right\rangle^{(1)} & =C_{1}^{(1)} F_{\theta_{1}} 2^{-k+3 / 2} \Gamma(-k+3 / 2) \\
& =C_{1}^{(1)} 2^{-k+3 / 2} \Gamma(-k+3 / 2)
\end{aligned}
$$

donde usamos que

$$
F_{\theta_{1}}=\frac{1}{2^{3 / 2} \Gamma(3 / 2)} \int_{0}^{\infty} t^{1 / 2} \mathrm{e}^{-t} \mathrm{~d} t=1 .
$$

Los únicos valores que puede tomar $k$ son 0 y 1 . Para $k=0$ obtenemos la constante de normalización: $C_{1}^{(1)}=1 / 2^{3 / 2}(1 / 2)$ !, mientras que para $k=1$ tenemos que

$$
\left\langle x_{1}^{-k}\right\rangle^{(1)}=\left(\frac{1}{2}\right)^{k} \frac{\left(\frac{1}{2}-k\right) !}{\left(\frac{1}{2}\right) !} \text {. }
$$

\subsubsection{Caso $N=2$}

En este caso, $S_{2}=\{\iota, \sigma\}$, tal que $\sigma_{1}=\iota, \sigma$ con $\iota(a)=a(a=1,2)$, $\sigma(1)=2$ y $\sigma(2)=1$; por tanto, $\gamma_{a}=\sigma_{1}(a)$. La ecuación (5.31) para $N=2$ da

$$
\begin{aligned}
\left\langle x_{1}^{-k_{1}} x_{2}^{-k_{2}}\right\rangle^{(1)}= & C_{2}^{(1)} 2^{5-k_{1}-k_{2}}\left[\left(F_{\theta_{\iota, 2}}-F_{\theta_{\sigma, 2}^{\sigma}}\right) \Gamma\left(2-k_{1}\right) \Gamma\left(3-k_{2}\right)\right. \\
& \left.+\left(F_{\theta_{\sigma, 2}^{\iota}}-F_{\theta_{\iota, 2}^{\sigma}}\right) \Gamma\left(3-k_{1}\right) \Gamma\left(2-k_{2}\right)\right] .
\end{aligned}
$$

Aquí, la ecuación (5.22) conduce a $\alpha_{a}^{\eta}=\sigma_{1}(a)-k_{\eta(a)}+1$ y la ecuación (5.25) da

$$
\theta_{\eta, N}^{\sigma_{1}}=\left[\sigma_{1}(i)-k_{\eta(1)}+1, \sigma_{1}(2)-k_{\eta(2)}+1,1 / 2,1 / 2\right] .
$$

De acuerdo con la ecuación (D.12) del apéndice D, podemos determinar los

coeficientes $F_{\theta_{\eta, N}}^{\sigma_{1}}$ por medio de la distribución binomial negativa $N B_{\alpha_{2}, p_{2}}$ con parámetros $\alpha_{2}$ y $p_{2}=1 / 2^{1}$. Para el caso particular de $N=2$

$$
F_{\theta_{\eta, 2}}^{\sigma_{1}}=1-\sum_{\ell=0}^{\sigma_{1}(1)-k_{\eta(1)}} N B_{\sigma_{1}(2)-k_{\eta(2)}+1,1 / 2}(\ell)
$$

\footnotetext{
${ }^{1}$ La distribución binomial negativa sólo se puede usar cuando $N$ es un número par.
} 
para cualquier $\eta$ y $\sigma_{1} \in S_{2}$ y $k_{a}=0,1$.

Para el caso particular de $k_{1}=k_{2}=0$ tenemos que

$$
\begin{aligned}
& F_{\theta_{2}^{\iota}}=1-\sum_{\ell=0}^{\iota(1)} N B_{\iota(2)+1,1 / 2}(\ell)=1-\sum_{\ell=0}^{1} N B_{3,1 / 2}(\ell)=\frac{11}{16}, \\
& F_{\theta_{2}^{\sigma}}=1-\sum_{\ell=0}^{\sigma(1)} N B_{\sigma(2)+1,1 / 2}(\ell)=1-\sum_{\ell=0}^{2} N B_{2,1 / 2}(\ell)=\frac{5}{16},
\end{aligned}
$$

donde hemos usado la definición (D.4), y la constante de normalización es $C_{2}^{(1)}=1 / 48$.

De manera similar, para $k_{1}=k=1$ y $k_{2}=0$,

$$
F_{\theta_{\iota, 2}^{\iota}}=\frac{7}{8}, \quad F_{\theta_{\sigma, 2}^{\iota}}=\frac{1}{2}, \quad F_{\theta_{\iota, 2}^{\sigma}}=\frac{1}{2}, \quad \mathrm{y} \quad F_{\theta_{\sigma, 2}^{\sigma}}=\frac{1}{8},
$$

tal que

$$
\left\langle x_{1}^{-k}\right\rangle^{(1)}=\left(\frac{1}{2}\right)^{k} \frac{(1-k) !}{1 !} K_{2}^{(1)}(k, 0),
$$

donde

$$
K_{2}^{(1)}(k, 0)=1 \quad \text { para } \quad k=1 .
$$

Para $k_{1}=k_{2}=k=1$ tenemos que

$$
F_{\theta_{\iota, 2}^{\iota}}=\frac{3}{4}, \quad F_{\theta_{\sigma, 2}^{\iota}}=\frac{3}{4}, \quad F_{\theta_{\iota, 2}^{\sigma}}=\frac{1}{4}, \quad \text { y } \quad F_{\theta_{\sigma, 2}^{\sigma}}=\frac{1}{4}
$$

y podemos escribir el correspondiente momento como

$$
\left\langle x_{1}^{-k} x_{2}^{-k}\right\rangle^{(1)}=\left(\frac{1}{2}\right)^{2 k} \frac{(1-k) !}{1 !} \frac{\left(\frac{3}{2}-k\right) !}{\frac{3}{2} !} .
$$

Para $\beta=1$, los cálculos se vuelven mucho más complicados para valores de $N$ más grandes que 2 . Sin embargo, algunos momentos se pueden obtener de manera numérica, algunos de los cuales se muestran más adelante, en la sección 5.5.

\subsection{Cálculos explícitos para $\beta=2$}

De la ecuación (5.15) tenemos que $\gamma_{a}=N-2+\sigma_{1}(a)+\sigma_{2}(a)(a=$ $1, \ldots, N)$ y la ecuación (5.29) se puede escribir como

$$
A_{N}^{(2)}\left(k_{1}, \ldots, k_{N}\right)=\sum_{\sigma_{1}, \sigma_{2} \in S_{N}} \operatorname{sgn}\left(\sigma_{1} \sigma_{2}\right) \prod_{a=1}^{N}\left[N-2+\sigma_{1}(a)+\sigma_{2}(a)-k_{a}\right] ! ;
$$


la constante de normalización es $C_{N}^{(2)}=1 / A_{N}^{(2)}$, con $A_{N}^{(2)}=A_{N}^{(2)}(0, \ldots, 0), \mathrm{y}$ los momentos estarán dados por la ecuación (5.28).

\subsubsection{Caso $N=1$}

En este caso especial, $\gamma_{1}=\sigma_{1}+\sigma_{2}-1$, tal que la ecuación (5.45) conduce a

$$
A_{1}^{(2)}(k)=\sum_{\sigma_{1}, \sigma_{2} \in S_{1}} \operatorname{sgn}\left(\sigma_{1} \sigma_{2}\right)\left(\sigma_{1}+\sigma_{2}-k-1\right) !=(1-k) !
$$

Ya que $N \beta / 2+1=2$, de acuerdo con la observación 2 el máximo valor de $k$ es 1 . La constante de normalización para $k=0$ es $C_{1}^{(2)}=1 / 1$ !. Por tanto, el único momento es

$$
\left\langle x_{1}^{-k}\right\rangle^{(2)}=\frac{(1-k) !}{1 !} \quad \text { para } \quad k=1 .
$$

\subsubsection{Caso $N=2$}

En este caso observamos que $\gamma_{a}=\sigma_{1}(a)+\sigma_{2}(a)(a=1,2)$ y la ecuación (5.45) se vuelve

$$
A_{2}^{(2)}\left(k_{1}, k_{2}\right)=\left(2-k_{1}\right) !\left(4-k_{2}\right) !+\left(4-k_{1}\right) !\left(2-k_{2}\right) !-2\left(3-k_{1}\right) !\left(3-k_{2}\right) !
$$

Aquí, $N \beta / 2+1=3$ tal que el máximo valor del momento negativo es 2 ; esto es, $k_{a}=0,1,2(a=1,2)$.

Para $k_{1}=k_{2}=0$ obtenemos la constante de normalización, $C_{2}^{(2)}=$ $1 / 2$ ! $\left(3 ! \cdot 2\right.$ ! $\cdot 1$ !). Los correspondientes momentos para $k_{1}=k$ y $k_{2}=0$ son

$$
\left\langle x_{1}^{-k}\right\rangle^{(2)}=\frac{(2-k) !}{2 !} K_{2}^{(2)}(k, 0),
$$

donde

$$
K_{2}^{(2)}(k, 0)=\frac{1}{3 !}[12-6(3-k)+(3-k)(4-k)] .
$$

También, para $k_{1}=k_{2}=k$ tenemos

$$
\left\langle x_{1}^{-k} x_{2}^{-k}\right\rangle^{(2)}=\frac{(3-k) !}{3 !} \frac{(2-k) !}{2 !} .
$$

El momento negativo restante es

$$
\left\langle x_{1}^{-2} x_{2}^{-1}\right\rangle^{(2)}=\frac{1 !}{3 !} .
$$




\subsubsection{Caso $N=3$}

Para $N=3, \gamma_{a}=\sigma_{1}(a)+\sigma_{2}(a)+1(a=1,2,3)$ y la ecuación $(5.45)$ se vuelve

$$
\begin{aligned}
& A_{3}^{(2)}\left(k_{1}, k_{2}, k_{3}\right)=\left(3-k_{1}\right) !\left(5-k_{2}\right) !\left(7-k_{3}\right) ! \\
& +\left(3-k_{1}\right) !\left(7-k_{2}\right) !\left(5-k_{3}\right) ! \\
& +\left(5-k_{1}\right) !\left(3-k_{2}\right) !\left(7-k_{3}\right) \text { ! } \\
& +\left(5-k_{1}\right) !\left(7-k_{2}\right) !\left(3-k_{3}\right) ! \\
& +\quad\left(7-k_{1}\right) !\left(3-k_{2}\right) !\left(5-k_{3}\right) \text { ! } \\
& +\left(7-k_{1}\right) !\left(5-k_{2}\right) !\left(3-k_{3}\right) \text { ! } \\
& \text { - } 2\left(3-k_{1}\right) !\left(6-k_{2}\right) !\left(6-k_{3}\right) \text { ! } \\
& \text { - } 2\left(6-k_{1}\right) !\left(3-k_{2}\right) !\left(6-k_{3}\right) \text { ! } \\
& \text { - } 2\left(6-k_{1}\right) !\left(6-k_{2}\right) !\left(3-k_{3}\right) \text { ! } \\
& \text { - } 2\left(4-k_{1}\right) !\left(4-k_{2}\right) !\left(7-k_{3}\right) \text { ! } \\
& \text { - } 2\left(4-k_{1}\right) !\left(7-k_{2}\right) !\left(4-k_{3}\right) \text { ! } \\
& \text { - } 2\left(7-k_{1}\right) !\left(4-k_{2}\right) !\left(4-k_{3}\right) ! \\
& +2\left(4-k_{1}\right) !\left(5-k_{2}\right) !\left(6-k_{3}\right) ! \\
& +2\left(4-k_{1}\right) !\left(6-k_{2}\right) !\left(5-k_{3}\right) ! \\
& +2\left(6-k_{1}\right) !\left(5-k_{2}\right) !\left(4-k_{3}\right) \text { ! } \\
& +2\left(5-k_{1}\right) !\left(4-k_{2}\right) !\left(6-k_{3}\right) \text { ! } \\
& +2\left(5-k_{1}\right) !\left(6-k_{2}\right) !\left(4-k_{3}\right) \text { ! } \\
& +2\left(6-k_{1}\right) !\left(4-k_{2}\right) !\left(5-k_{3}\right) \text { ! } \\
& \text { - } 6\left(5-k_{1}\right) !\left(5-k_{2}\right) !\left(5-k_{3}\right) \text { ! }
\end{aligned}
$$

El máximo valor que puede tomar $k_{a}(a=1,2,3)$ es 3 . Si evaluamos esta expresión con $k_{1}=k_{2}=k_{3}=0$ obtenemos $C_{3}^{(2)}=1 / 3 !(5 ! \cdot 4 ! \cdot 3 ! \cdot 2 ! \cdot 1$ !).

El resultado de los momentos para $k_{1}=k$ y $k_{2}=k_{3}=0$ está dado por

$$
\left\langle x_{1}^{-k}\right\rangle^{(2)}=\frac{(3-k) !}{3 !} K_{3}^{(2)}(k, 0,0)
$$

donde

$$
\begin{aligned}
K_{3}^{(2)}(k, 0,0) & =\frac{1}{6 !}[5 \cdot 6 !-4 \cdot 6 !(4-k)+150 \cdot 3 !(4-k)(5-k) \\
& -5 !(4-k)(5-k)(6-k)+3 !(4-k)(5-k)(6-k)(7-k)] .
\end{aligned}
$$


De manera similar, para $k_{1}=k_{2}=k$ y $k_{3}=0$ tenemos que

$$
\left\langle x_{1}^{-k} x_{2}^{-k}\right\rangle^{(2)}=\frac{(3-k) !}{3 !} \frac{(4-k) !}{4 !} K_{3}^{(2)}(k, k, 0),
$$

donde

$$
\begin{aligned}
K_{3}^{(2)}(k, k, 0) & =\frac{1}{6 !}[7 !-6 !(k-1)(5-k) \\
& \left.-4 \cdot 4 !(4-k)(5-k)(6-k)+3 !(4-k)(5-k)^{2}(6-k)\right] .
\end{aligned}
$$

Los momentos para $k_{1}=k_{2}=k_{3}=k$ están dados por

$$
\left\langle x_{1}^{-k} x_{2}^{-k} x_{3}^{-k}\right\rangle^{(2)}=\frac{(5-k) !}{5 !} \frac{(4-k) !}{4 !} \frac{(3-k) !}{3 !} .
$$

Los términos restantes se pueden evaluar directamente de la ecuación (5.53). Éstos son:

$$
\left\langle x_{1}^{-2} x_{2}^{-1}\right\rangle^{(2)}=\frac{3 !}{5 !}, \quad\left\langle x_{1}^{-3} x_{2}^{-1}\right\rangle^{(2)}=\frac{62}{6 !}, \quad \mathrm{y} \quad\left\langle x_{1}^{-3} x_{2}^{-2}\right\rangle^{(2)}=\frac{3}{5 !} ;
$$

también,

$$
\begin{aligned}
& \left\langle x_{1}^{-2} x_{2}^{-1} x_{3}^{-1}\right\rangle^{(2)}=\frac{6}{6 !},\left\langle x_{1}^{-2} x_{2}^{-2} x_{3}^{-1}\right\rangle^{(2)}=\frac{2 !}{6 !},\left\langle x_{1}^{-3} x_{2}^{-1} x_{3}^{-1}\right\rangle^{(2)}=\frac{10}{6 !} \\
& \left\langle x_{1}^{-3} x_{2}^{-2} x_{3}^{-1}\right\rangle^{(2)}=\frac{5}{2 \cdot 6 !} \\
& \left\langle x_{1}^{-3} x_{2}^{-2} x_{3}^{-2}\right\rangle^{(2)}=\frac{1}{2 \cdot 6 !}, \quad\left\langle x_{1}^{-3} x_{2}^{-3} x_{3}^{-1}\right\rangle^{(2)}=\frac{1}{4 \cdot 5 !}, \quad \mathrm{y} \\
& \left\langle x_{1}^{-3} x_{2}^{-3} x_{3}^{-2}\right\rangle^{(2)}=\frac{1}{4 \cdot 6 !} .
\end{aligned}
$$

\subsubsection{Caso $N=4$}

De la ecuación (5.15), $\gamma_{a}=\sigma_{1}(a)+\sigma_{2}(a)+2(a=1,2,3,4)$; en este caso la ecuación (5.45) conduce a

$$
\begin{aligned}
A_{4}^{(2)}\left(k_{1}, k_{2}, k_{3}, k_{4}\right) & =\left(4-k_{1}\right) !\left(6-k_{2}\right) !\left(8-k_{3}\right) !\left(10-k_{4}\right) ! \\
& -\left(4-k_{1}\right) !\left(6-k_{2}\right) !\left(9-k_{3}\right) !\left(9-k_{4}\right) ! \\
& -\left(4-k_{1}\right) !\left(7-k_{2}\right) !\left(7-k_{3}\right) !\left(10-k_{4}\right) !
\end{aligned}
$$




$$
\begin{aligned}
& +\left(4-k_{1}\right) !\left(7-k_{2}\right) !\left(9-k_{3}\right) !\left(8-k_{4}\right) ! \\
& +\quad\left(4-k_{1}\right) !\left(8-k_{2}\right) !\left(7-k_{3}\right) !\left(9-k_{4}\right) ! \\
& -\quad\left(4-k_{1}\right) !\left(8-k_{2}\right) !\left(8-k_{3}\right) !\left(8-k_{4}\right) ! \\
& -\quad\left(5-k_{1}\right) !\left(5-k_{2}\right) !\left(8-k_{3}\right) !\left(10-k_{4}\right) ! \\
& +\quad\left(5-k_{1}\right) !\left(5-k_{2}\right) !\left(9-k_{3}\right) !\left(9-k_{4}\right) ! \\
& +\quad\left(5-k_{1}\right) !\left(7-k_{2}\right) !\left(6-k_{3}\right) !\left(10-k_{4}\right) ! \\
& -\quad\left(5-k_{1}\right) !\left(7-k_{2}\right) !\left(9-k_{3}\right) !\left(7-k_{4}\right) ! \\
& -\quad\left(5-k_{1}\right) !\left(8-k_{2}\right) !\left(6-k_{3}\right) !\left(9-k_{4}\right) ! \\
& +\quad\left(5-k_{1}\right) !\left(8-k_{2}\right) !\left(8-k_{3}\right) !\left(7-k_{4}\right) ! \\
& +\quad\left(6-k_{1}\right) !\left(5-k_{2}\right) !\left(7-k_{3}\right) !\left(10-k_{4}\right) ! \\
& -\quad\left(6-k_{1}\right) !\left(5-k_{2}\right) !\left(9-k_{3}\right) !\left(8-k_{4}\right) ! \\
& -\quad\left(6-k_{1}\right) !\left(6-k_{2}\right) !\left(6-k_{3}\right) !\left(10-k_{4}\right) ! \\
& +\quad\left(6-k_{1}\right) !\left(6-k_{2}\right) !\left(9-k_{3}\right) !\left(7-k_{4}\right) ! \\
& +\quad\left(6-k_{1}\right) !\left(8-k_{2}\right) !\left(6-k_{3}\right) !\left(8-k_{4}\right) ! \\
& -\quad\left(6-k_{1}\right) !\left(8-k_{2}\right) !\left(7-k_{3}\right) !\left(7-k_{4}\right) ! \\
& -\quad\left(7-k_{1}\right) !\left(5-k_{2}\right) !\left(7-k_{3}\right) !\left(9-k_{4}\right) ! \\
& +\quad\left(7-k_{1}\right) !\left(5-k_{2}\right) !\left(8-k_{3}\right) !\left(8-k_{4}\right) ! \\
& +\quad\left(7-k_{1}\right) !\left(6-k_{2}\right) !\left(6-k_{3}\right) !\left(9-k_{4}\right) ! \\
& -\left(7-k_{1}\right) !\left(6-k_{2}\right) !\left(8-k_{3}\right) !\left(7-k_{4}\right) ! \\
& -\left(7-k_{1}\right) !\left(7-k_{2}\right) !\left(6-k_{3}\right) !\left(8-k_{4}\right) ! \\
& +\quad\left(7-k_{1}\right) !\left(7-k_{2}\right) !\left(7-k_{3}\right) !\left(7-k_{4}\right) ! \\
& +\quad\left(\text { permutaciones de } k_{1}, k_{2}, k_{3}, k_{4}\right) .
\end{aligned}
$$

Esta ecuación está bien definida, ya que el máximo valor permitido para $k_{a}$ $(a=1,2,3,4)$ es 4 .

Aunque el cálculo de $A_{4}^{(2)}\left(k_{1}, k_{2}, k_{3}, k_{4}\right)$ para un conjunto arbitrario de valores de las $k_{a}$ 's no es difícil, este consiste de muchos términos que no es fácil de seguir. Sin embargo, existen dos cantidades que son claramente realizables: uno para $k_{a}=0 \mathrm{y}$ el otro para $k_{a}=k$. Esto es debido al hecho de que las permutaciones de las $k_{a}$ 's en la ecuación (5.62) dan los mismos términos que hemos escrito explícitamente. La primera cantidad da la constante de normalización, $C_{4}^{(2)}=1 / 4 !(7 ! \cdot 6 ! \cdot 5 ! \cdot 4 ! \cdot 3 ! \cdot 2 ! \cdot 1 !)$. La segunda cantidad es el momento

$$
\left\langle x_{1}^{-k} x_{2}^{-k} x_{3}^{-k} x_{4}^{-k}\right\rangle^{(2)}=\frac{(7-k) !}{7 !} \frac{(6-k) !}{6 !} \frac{(5-k) !}{5 !} \frac{(4-k) !}{4 !} .
$$


Cualquier otro momento es difícil de calcular aritméticamente, sin embargo, podemos llegar a los siguientes resultados:

$$
\begin{aligned}
& \left\langle x_{1}^{-1}\right\rangle^{(2)}=\frac{3 !}{4 !},\left\langle x_{1}^{-2}\right\rangle^{(2)}=\frac{8 \cdot 2 !}{5 !}, \quad\left\langle x_{1}^{-3}\right\rangle^{(2)}=\frac{16 \cdot 3 !}{6 !}, \quad \mathrm{y} \\
& \left\langle x_{1}^{-4}\right\rangle^{(2)}=\frac{59 \cdot 4 !}{7 !} \\
& \left\langle x_{1}^{-1} x_{2}^{-1}\right\rangle^{(2)}=\frac{6}{5 !},\left\langle x_{1}^{-2} x_{2}^{-2}\right\rangle^{(2)}=\frac{3}{6 !}\left(\frac{8 \cdot 9}{6 \cdot 7}\right), \quad \mathrm{y} \\
& \left\langle x_{1}^{-3} x_{2}^{-3}\right\rangle^{(2)}=\frac{8 \cdot 5 !}{9 !} \\
& \left\langle x_{1}^{-1} x_{2}^{-1} x_{3}^{-1}\right\rangle^{(2)}=\frac{1}{5 !}, \quad\left\langle x_{1}^{-2} x_{2}^{-2} x_{3}^{-2}\right\rangle^{(2)}=\frac{1}{7 !}, \quad \mathrm{y} \\
& \left\langle x_{1}^{-3} x_{2}^{-3} x_{3}^{-3}\right\rangle^{(2)}=\frac{51}{10 !} .
\end{aligned}
$$

\subsection{Cálculos explícitos para $\beta=4$}

Para $\beta=4$, la ecuación (5.15) conduce a $\gamma_{a}=2 N-4+\sigma_{1}(a)+\sigma_{2}(a)+$ $\sigma_{3}(a)+\sigma_{4}(a)(a=1, \ldots, N)$, tal que la ecuación (5.29) da

$$
A_{N}^{(4)}\left(k_{1}, \ldots, k_{N}\right)=\sum_{\sigma_{1}, \sigma_{2}, \sigma_{3} \sigma_{4} \in S_{N}} \operatorname{sgn}\left(\sigma_{1} \sigma_{2} \sigma_{3} \sigma_{4}\right) \prod_{i=1}^{N} \frac{\left(\gamma_{i}-k_{i}\right) !}{2^{\gamma_{i}-k_{i}}}
$$

La constante de normalización está dada por $C_{N}^{(4)}=2^{N} / A_{N}^{(4)}$ y los momentos están dados por la ecuación (5.28).

\subsubsection{Caso $N=1$}

En este caso, $\gamma=\left(\sigma_{1}+\sigma_{2}+\sigma_{3}+\sigma_{4}-k-2\right)$ ! y la ecuación $(5.67)$ se puede escribir como

$$
A_{1}^{(4)}(k)=2^{k} \frac{(2-k) !}{2^{2}},
$$

tal que $A_{1}^{(4)}=1 / 2$ y $C_{1}^{(4)}=2^{2}$. Por lo tanto,

$$
\left\langle x_{1}^{-k}\right\rangle^{(4)}=2^{k} \frac{(2-k) !}{2 !} \quad \text { para } \quad k=1,2 .
$$




\subsubsection{Caso $N=2$}

Aquí, $\sigma_{i} \in S_{2}(i=1,2,3,4)$ y

$$
\begin{aligned}
A_{2}^{(4)}\left(k_{1}, k_{2}\right) & =2^{k_{1}+k_{2}-12}\left[\left(4-k_{1}\right) !\left(8-k_{2}\right) !-4\left(5-k_{1}\right) !\left(7-k_{2}\right) !\right. \\
& \left.+3\left(6-k_{1}\right) !\left(6-k_{2}\right) !\right]+\left(\text { permutaciones de } k_{1} \mathrm{y} k_{2}\right) .
\end{aligned}
$$

Para $k_{1}=k_{2}=0$, tenemos que $C_{2}^{(4)}=2^{14} / 3 !(5 ! \cdot 4 ! \cdot 3 ! \cdot 2 ! \cdot 1 !)$, mientras que para $k_{1}=k_{2}=k$,

$$
\left\langle x_{1}^{-k} x_{2}^{-k}\right\rangle^{(4)}=2^{2 k} \frac{(6-k) !}{6 !} \frac{(4-k) !}{4 !}, \quad \text { para } \quad k=1,2,3,4 .
$$

También, para $k_{1}=k$ y $k_{2}=0$, obtenemos

$$
\left\langle x_{1}^{-k}\right\rangle^{(4)}=2^{k} \frac{(4-k) !}{4 !} K_{2}^{(4)}(k, 0),
$$

donde

$$
\begin{aligned}
K_{2}^{(4)}(k, 0) & =\frac{1}{6 ! \cdot 3 ! \cdot 2 !}[8 !+2 \cdot 6 !(5-k)(4-3 k) \\
& -4 !(12+k)(7-k)(6-k)(5-k)] .
\end{aligned}
$$

De la ecuación (5.70) es fácil ver que los momentos restantes están dados por

$$
\begin{aligned}
& \left\langle x_{1}^{-2} x_{2}^{-1}\right\rangle^{(4)}=\frac{1}{9}, \quad\left\langle x_{1}^{-3} x_{2}^{-1}\right\rangle^{(4)}=\frac{7}{45}, \quad \text { y } \quad\left\langle x_{1}^{-3} x_{2}^{-2}\right\rangle^{(4)}=\frac{2}{45} \\
& \left\langle x_{1}^{-4} x_{2}^{-1}\right\rangle^{(4)}=\frac{22}{45}, \quad\left\langle x_{1}^{-4} x_{2}^{-2}\right\rangle^{(4)}=\frac{2}{15}, \quad \text { y } \quad\left\langle x_{1}^{-4} x_{2}^{-3}\right\rangle^{(4)}=\frac{2}{45} .
\end{aligned}
$$

\subsection{Expresiones generales para los momentos con- juntos}

El conjunto de ecuaciones (5.34), (5.41), (5.47), (5.49), (5.54), (5.69), y (5.72); (5.44), (5.51), (5.56), y (5.71); (5.58) y (5.63), sugiere una expresión general para los momentos conjuntos para cualquier simetría y número de canales, la cual es

$$
\left\langle q_{1}^{k} \cdots q_{m}^{k}\right\rangle^{(\beta)}=\left[\prod_{n=N}^{N+m-1}\left(\frac{\beta}{2}\right)^{k} \frac{\left(\frac{\beta n}{2}-k\right) !}{\left(\frac{\beta n}{2}\right) !}\right] K_{N}^{(\beta)}(\underbrace{k, \ldots, k}_{m}, \underbrace{0, \ldots, 0}_{N-m}),
$$


para $m \leq N$, donde $K_{N}^{(\beta)}(\underbrace{k, \ldots, k}_{m}, \underbrace{0, \ldots, 0}_{N-m})$ tiene una expresión particular para cada valor de $N$ y $m$, como se puede ver en las ecuaciones (5.42), (5.50), (5.55), (5.57), y (5.73). Es difícil obtener, analíticamente, una expresión cerrada para esta cantidad, pero se reduce a 1 para $m=N$, como también sugieren las ecuaciones (5.34), (5.44), (5.47), (5.51), (5.58), (5.63), (5.69), y (5.71),

$$
K_{N}^{(\beta)}(k, \ldots, k)=1,
$$

así también, para $k=1$ y $m \leq N$,

$$
K_{N}^{(\beta)}(\underbrace{1, \ldots, 1}_{m}, \underbrace{0, \ldots, 0}_{N-m})=1
$$

como puede verse mediante la evaluación directa de las ecuaciones arriba mencionadas.

Un caso de interés particular es el del $k$-ésimo momento para $m=1$ (una sola variable); encontramos que

$$
\left\langle q_{1}^{k}\right\rangle^{(\beta)}=\left(\frac{\beta}{2}\right)^{k} \frac{\left(\frac{\beta N}{2}-k\right) !}{\left(\frac{\beta N}{2}\right) !} K_{N}^{(\beta)}(k, 0, \ldots, 0), \quad \text { para } \quad k<\frac{\beta N}{2}+1 .
$$

donde el factor $K_{N}^{(\beta)}(k, 0, \ldots, 0)$ está dado por la siguiente expresión:

$$
K_{N}^{(\beta)}(k, 0, \ldots, 0)=\frac{k ! N^{k-1} N !}{(N+k-1) !} \quad \text { para } \quad k=1,2,3
$$

independiente de $\beta$.

De las ecuaciones (5.79) y (5.80) obtenemos los siguientes promedios:

$$
\left\langle q_{1}\right\rangle^{(\beta)}=\frac{1}{N}, \quad\left\langle q_{1}^{2}\right\rangle^{(\beta)}=\frac{\beta}{2} \frac{2}{\left(\frac{\beta N}{2}-1\right)(N+1)},
$$

y

$$
\left\langle q_{1}^{3}\right\rangle^{(\beta)}=\left(\frac{\beta}{2}\right)^{2} \frac{3 ! N}{\left(\frac{\beta N}{2}-1\right)\left(\frac{\beta N}{2}-2\right)(N+1)(N+2)},
$$

los cuales están en acuerdo con los de la referencia [65] para $\beta=2$ obtenidos en el límite semi-clásico, y los de la referencia [66] para cualquier $\beta$ 
y $N$. Ambos resultados en la ecuación (5.81) se reportaron en las referencias $[9,20]$ para $\beta=1$ y 2 . Otro caso que se puede obtener fácilmente de las ecuaciones (5.76) y (5.78), que es de interés particular, es

$$
\left\langle q_{1} q_{2}\right\rangle^{(\beta)}=\frac{1}{N(N+1)} .
$$

el cual fue reportado en la referencia [67] para $\beta=2$.

Como el tiempo de retardo de Wigner es $\tau_{W}=\tau_{H} \sum_{i}^{N} q_{i} / N$, su media y su varianza se pueden calcular de las ecuaciones (5.81) y (5.83) para cualquier $\beta$ y $N$; los resultados son

$$
\tau_{W}=\frac{\tau_{H}}{N}, \quad \frac{\left\langle\tau_{W}^{2}\right\rangle-\left\langle\tau_{W}\right\rangle^{2}}{\left\langle\tau_{W}\right\rangle^{2}}=\frac{2}{\left(\frac{\beta N}{2}-1\right)(N+1)},
$$

el cual para $\beta=2$ se reduce a los de las referencias [36, 40].

De las ecuaciones (5.51), (5.56), (5.57), la segunda ecuación en (5.65), y la ecuación (5.71), es factible hallar que

$$
K_{N}^{(\beta)}(2,2,0, \ldots, 0)=\frac{2 N(2 N+1)}{(N+2)(N+3)} .
$$

Aunque no tenemos resultados explícitos para $\beta=1$, esta expresión la verificamos numéricamente. Este resultado nos permite obtener

$$
\left\langle q_{1}^{2} q_{2}^{2}\right\rangle^{(\beta)}=\left(\frac{\beta}{2}\right)^{4} \frac{\left(\frac{\beta N}{2}-2\right) !}{\left(\frac{\beta N}{2}\right) !} \frac{\left[\frac{\beta(N+1)}{2}-2\right] !}{\left[\frac{\beta(N+1)}{2}\right] !} K_{N}^{(\beta)}(2,2,0, \ldots, 0) .
$$

En la Tabla 5.1 resumimos algunos momentos expresados en las ecuaciones (5.52), (5.59), (5.60), (5.74), y otros que obtuvimos de manera numérica. Estos se puede generalizar fácilmente a

$$
\left\langle q_{1}^{2} q_{2}\right\rangle^{(\beta)}=\left(\frac{\beta}{2}\right)^{3} \frac{\left(\frac{\beta N}{2}-2\right) !}{\left(\frac{\beta N}{2}\right) !} \frac{\left[\frac{\beta(N+1)}{2}-1\right] !}{\left[\frac{\beta(N+1)}{2}\right] !} K_{N}^{(\beta)}(2,1,0,0, \ldots, 0),
$$

con

$$
\begin{gathered}
K_{N}^{(\beta)}(2,1,0,0, \ldots, 0)=\frac{2 N}{N+2} \\
\left\langle q_{1}^{3} q_{2}\right\rangle^{(\beta)}=\left(\frac{\beta}{2}\right)^{4} \frac{\left(\frac{\beta N}{2}-3\right) !}{\left(\frac{\beta N}{2}\right) !} \frac{\left[\frac{\beta(N+1)}{2}-1\right] !}{\left[\frac{\beta(N+1)}{2}\right] !} K_{N}^{(\beta)}(3,1,0,0, \ldots, 0),
\end{gathered}
$$




\begin{tabular}{|c|c|c|c|c|c|}
\hline & $N$ & 2 & 3 & 4 & 5 \\
\hline \multirow{5}{*}{$\beta=1$} & $\left\langle q_{1}^{2} q_{2}\right\rangle$ & - & - & $\frac{4 \cdot 6 \cdot 1 !}{6 !}$ & - \\
\hline & $\left\langle q_{1}^{3} q_{2}\right\rangle$ & - & - & 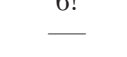 & - \\
\hline & $\left\langle q_{1}^{3} q_{2}^{2}\right\rangle$ & - & - & - & - \\
\hline & $\left\langle q_{1}^{2} q_{2} q_{3}\right\rangle$ & - & $\frac{2 \cdot 0 !}{5 !}$ & $\frac{2 \cdot 12 \cdot 1 !}{7 !}$ & - \\
\hline & $\left\langle q_{1}^{2} q_{2}^{2} q_{3}\right\rangle$ & - & & $\frac{2 \cdot 36 \cdot 1 !}{8 !}$ & - \\
\hline \multirow{4}{*}{$\beta=2$} & $\left\langle q_{1}^{2} q_{2}\right\rangle$ & $\frac{2 \cdot 2 \cdot 0 !}{4 !}$ & $\begin{array}{l}\frac{2 \cdot 3 \cdot 1 !}{5 !} \\
2 \cdot 31 \cdot 0 !\end{array}$ & $\begin{array}{r}\frac{2 \cdot 4 \cdot 2 !}{6 !} \\
2 \cdot 53 \cdot 1 ! \\
\end{array}$ & $\begin{array}{r}\frac{2 \cdot 5 \cdot 3 !}{7 !} \\
2 \cdot 81 \cdot 2 !\end{array}$ \\
\hline & $\begin{array}{l}\left\langle q_{1} q_{2}\right\rangle \\
\left\langle a_{1}^{3} a_{2}^{2}\right\rangle\end{array}$ & - & $\overline{6 \cdot 3 \cdot 7 \cdot 0 !}$ & $\overline{6 \cdot 4 \cdot \dot{9} \cdot 1 !}$ & $8.8 !$ \\
\hline & $\left\langle q_{1}^{2} q_{2} q_{3}\right\rangle$ & - & $\frac{2 \cdot 3 !}{6 !}$ & $\frac{2 \cdot 4 ! 2 !}{7 !}$ & $\frac{9 ! 9 !}{2 \cdot 3 !}$ \\
\hline & $\left\langle q_{1}^{2} q_{2}^{2} q_{3}\right\rangle$ & 一 & $\frac{2 \cdot 7 ! \cdot 1 !}{7 !}$ & $\frac{2 \cdot 9 ! 2 !}{8 ! !}$ & $\frac{2 \cdot 11 \cdot 3 !}{9 !}$ \\
\hline \multirow{4}{*}{$\beta=4$} & $\left\langle q_{1}^{2} q_{2}\right\rangle$ & $\frac{4 \cdot 2 \cdot 1 !}{3 \cdot 4 !}$ & $\begin{array}{c}\frac{4 \cdot 3 \cdot 2 !}{5 \cdot 5 !} \\
4 \cdot 3 \cdot 10 \cdot 1 !\end{array}$ & $\frac{4 \cdot 4 \cdot 3 !}{7 \cdot 6 !}$ & $\frac{4 \cdot 5 \cdot 4 !}{9 \cdot 7 !}$ \\
\hline & $\left\langle q_{1}^{\circ} q_{2}\right\rangle$ & $\frac{3 \cdot 5 !}{6 \cdot 4 \cdot 4 \cdot 0 !}$ & $\begin{array}{c}\frac{5 \cdot 6 !}{6 \cdot 4 \cdot 9 ! 1 !} \\
\end{array}$ & 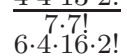 & $\frac{9 \cdot 8 !}{6 \cdot 4 \cdot 25 \cdot 3 !}$ \\
\hline & $\begin{array}{c}\left\langle q_{1} q_{2}\right\rangle \\
\left\langle q_{1}^{2} q_{2} q_{3}\right\rangle\end{array}$ & $\begin{array}{l}3 \cdot 6 ! \\
-\end{array}$ & $\begin{array}{r}\frac{5 \cdot 7 !}{4 \cdot 3 \cdot 2 !} \\
\frac{4 \cdot 6 !}{6 !}\end{array}$ & $\frac{7 \cdot 8 !}{\frac{4 \cdot 4 \cdot 3 !}{7.7 !}}$ & $\begin{array}{c}9 \cdot 9 ! \\
4 \cdot 5 \cdot 4 ! \\
0.9 !\end{array}$ \\
\hline & $\left\langle q_{1}^{2} q_{2}^{2} q_{3}\right\rangle$ & - & $\frac{8 \cdot 3 \cdot 6 !}{5 \cdot 7 !}$ & $\frac{8 \cdot 7 \cdot 7 \cdot 3 !}{7 \cdot 8 !}$ & $\frac{8 \cdot 5 \cdot 4 !}{9 \cdot 9 !}$ \\
\hline
\end{tabular}

Tabla 5.1: Resumen de resultados de otros momentos para $\beta=1,2,4$, algunos de los cuales se obtuvieron numéricamente.

donde

$$
\begin{gathered}
K_{N}^{(\beta)}(3,1,0,0, \ldots, 0)=\frac{2\left[3 N^{2}+N+(2-\beta / 2)\right]}{(N+3)(N+2)} \\
\left\langle q_{1}^{3} q_{2}^{2}\right\rangle^{(\beta)}=\left(\frac{\beta}{2}\right)^{5} \frac{\left(\frac{\beta N}{2}-3\right) !}{\left(\frac{\beta N}{2}\right) !} \frac{\left[\frac{\beta(N+1)}{2}-2\right] !}{\left[\frac{\beta(N+1)}{2}\right] !} K_{N}^{(\beta)}(3,2,0,0, \ldots, 0),
\end{gathered}
$$

con

$$
\begin{aligned}
& K_{N}^{(\beta)}(3,2,0,0, \ldots, 0)=\frac{6 N^{2}(2 N+1)}{(N+4)(N+3)(N+2)} \\
&\left\langle q_{1}^{2} q_{2} q_{3}\right\rangle^{(\beta)}=\left(\frac{\beta}{2}\right)^{4} \frac{\left(\frac{\beta N}{2}-2\right) !}{\left(\frac{\beta N}{2}\right) !} \frac{\left[\frac{\beta(N+1)}{2}-1\right] !}{\left[\frac{\beta(N+1)}{2}\right] !} \frac{\left[\frac{\beta(N+2)}{2}-1\right] !}{\left[\frac{\beta(N+2)}{2}\right] !} \\
& \times K_{N}^{(\beta)}(2,1,1,0, \ldots, 0),
\end{aligned}
$$

donde

$$
K_{N}^{(\beta)}(2,1,1,0, \ldots, 0)=\frac{2 N}{N+3}
$$




\begin{tabular}{c|cccc}
$N$ & 1 & 2 & 3 & 4 \\
\hline$C_{N}^{(1)}$ & $\frac{(1 / 2)^{3 / 2}}{(1 / 2) !}$ & $\frac{1}{48}$ & $\frac{(1 / 2)^{3 / 2}}{(1 / 2) ! \cdot 180 \cdot 48}$ & $\frac{1}{53760 \cdot 180 \cdot 48}$ \\
\hline$C_{N}^{(2)}$ & $\frac{1}{1 !(1 !)}$ & $\frac{1}{2 !(3 ! \cdot 2 ! \cdot 1 !)}$ & $\frac{1}{3 !(5 ! \cdot 4 ! \cdot 3 ! \cdot 2 ! \cdot 1 !)}$ & $\frac{1}{4 !(7 ! \cdot 6 ! \cdot 5 ! \cdot 4 ! \cdot 3 ! \cdot 2 ! \cdot 1 !)}$ \\
\hline$C_{N}^{(4)}$ & $\frac{2^{4}}{2 !(2 ! \cdot 0 !)}$ & $\frac{2^{16}}{4 !(6 ! \cdot 4 ! \cdot 2 ! \cdot 0 !)}$ & $\frac{2^{36}}{6 !(10 ! \cdot 8 ! \cdot 6 ! \cdot 4 ! \cdot 2 ! \cdot 0 !)}$ & $\frac{2^{64}}{8 !(14 ! \cdot 12 ! \cdot 10 ! \cdot 8 ! \cdot 6 ! \cdot 4 ! \cdot 2 ! \cdot 0 !)}$ \\
\hline
\end{tabular}

Tabla 5.2: Resumen de los resultados para la constante de normalización $C_{N}^{(\beta)}$ de la distribución de Laguerre para cada clase de simetría $\beta=1,2$ y 4 .

y

$$
\begin{aligned}
\left\langle q_{1}^{2} q_{2}^{2} q_{3}\right\rangle^{(\beta)}= & \left(\frac{\beta}{2}\right)^{5} \frac{\left(\frac{\beta N}{2}-2\right) !}{\left(\frac{\beta N}{2}\right) !} \frac{\left[\frac{\beta(N+1)}{2}-2\right] !}{\left[\frac{\beta(N+1)}{2}\right] !} \frac{\left[\frac{\beta(N+2)}{2}-1\right] !}{\left[\frac{\beta(N+2)}{2}\right] !} \\
& \times K_{N}^{(\beta)}(2,2,1,0, \ldots, 0)
\end{aligned}
$$

con

$$
K_{N}^{(\beta)}(2,2,1,0, \ldots, 0)=\frac{2 N(2 N+1)}{(N+4)(N+3)}
$$

Los resultados de las ecuaciones (5.86), (5.89) y (5.93) fueron reportados en la referencia [67] para el caso $\beta=2$.

\subsection{Constante de normalización de la distribución de Laguerre}

La constante de normalización $C_{N}^{(\beta)}$ de la distribución de Laguerre, ecuación (2.9) no se conocía aunque esta distribución ha sido usada ampliamente. Sin embargo, los resultados que hemos obtenido, tanto analítica como numéricamente, nos permiten determinar esta constante. Un resumen de estos resultados se muestran en la Tabla 5.2. Lo primero que notamos es que los resultados para $\beta=2$ sugieren una dependencia general con respecto a $N$; esto es [68]

$$
C_{N}^{(2)}=\frac{1}{N !} \prod_{n=0}^{2 N-1} \frac{1}{n !}
$$



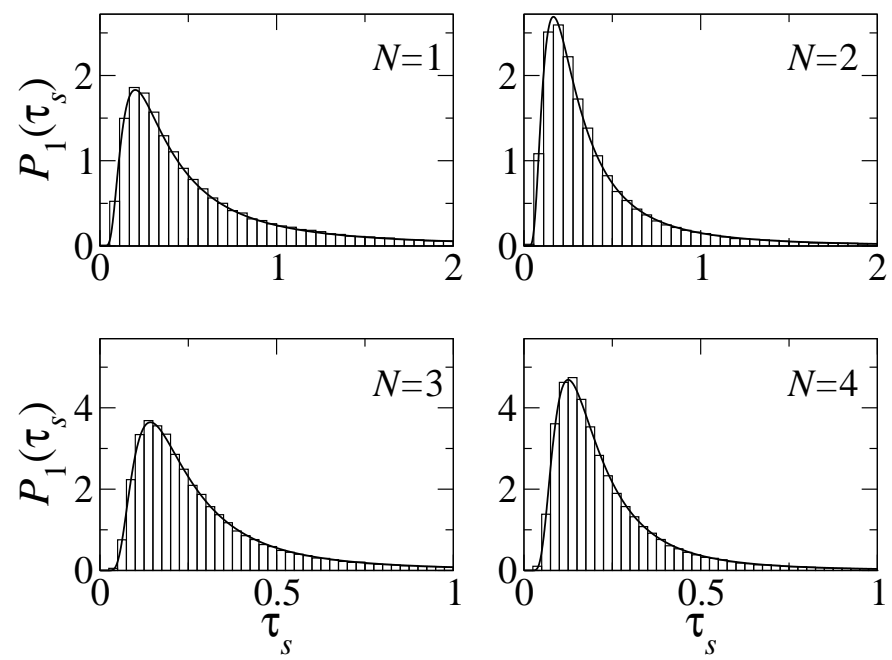

Figura 5.1: Comparación entre la simulación numérica (histogramas) y la teoría (líneas continuas), ecuación (5.102), para la distribución de $\tau_{s}$ para el caso $\beta=1$.

la cual da un indicio para las otras clases de simetría. Por ejemplo, para $\beta=4$ la constante de normalización se puede escribir como

$$
C_{N}^{(4)}=\frac{2^{(2 N)^{2}}}{(2 N) !} \prod_{n=0}^{2 N-1} \frac{1}{(2 n) !} .
$$

La dependencia en $N$ de esta constante para $\beta=1$ es más complicada que las correspondientes para $\beta=2$ y 4 , pero se puede obtener de una manera similar; el resultado es [68]

$$
C_{N}^{(1)}=\frac{\left[\left(\frac{1}{2}\right) !\right]^{N}}{2^{N(N+1 / 2)}\left(\frac{N}{2}\right) !} \prod_{n=0}^{2 N-1} \frac{1}{\left(\frac{n}{2}\right) !} .
$$

De estas expresiones es directo proponer el resultado general [68]

$$
C_{N}^{(\beta)}=\frac{\left[\left(\frac{\beta}{2}\right)^{\beta(N-1 / 2)+1}\left(\frac{\beta}{2}\right) !\right]^{N}}{\left(\frac{\beta N}{2}\right) !} \prod_{n=0}^{2 N-1} \frac{1}{\left(\frac{\beta n}{2}\right) !} .
$$

Lo que resulta ser muy interesante de este resultado es la similitud con la constante de normalización de la densidad de probabilidad conjunta de los valores propios del Hamiltoniano para los ensembles Gaussianos [32]. 

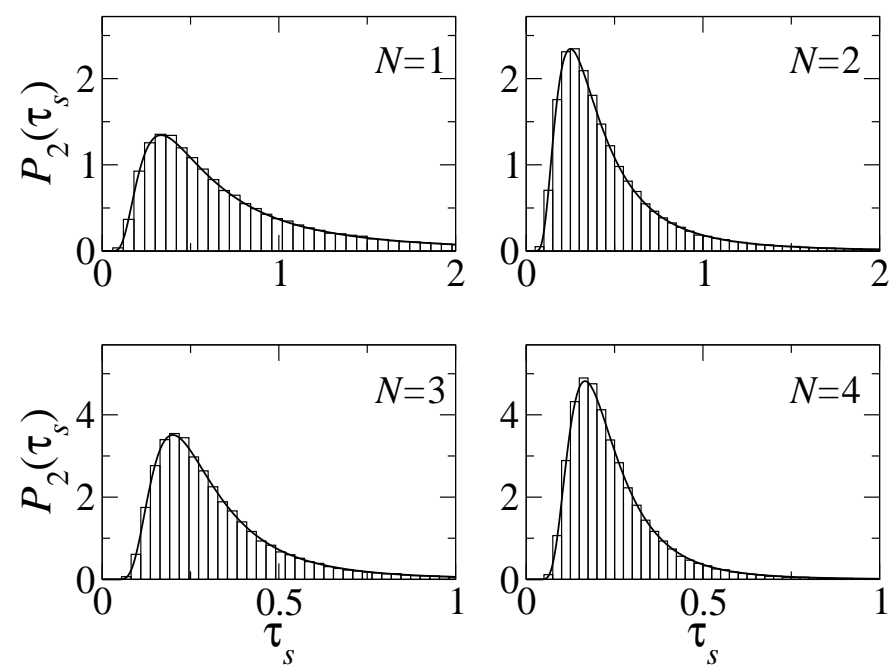

Figura 5.2: Lo mismo que en la figura 5.1 pero para el caso $\beta=2$.

\subsection{Tiempos de retardo parciales}

Un cosa que es bastante interesante del resultado expresado en la ecuación (5.79) es que el $k$-ésimo momento de un tiempo de retardo propio difiere del de un tiempo de retardo parcial, como puede verse si comparamos este resultado para $\beta=2$ con el resultado correspondiente de las referencias $[36,40]$. La diferencia cuantitativa está dada por el factor $K_{N}^{(\beta)}(k, 0, \ldots, 0)$ que viene de la repulsión de niveles para los tiempos de retardo propios. Mas aún, esto nos permite generalizar el $k$-ésimo momento de un tiempo parcial para cualquier $\beta$ y $N$; es decir

$$
\left\langle\tau_{s}^{k}\right\rangle^{(\beta)}=\left(\frac{\beta}{2}\right)^{k} \frac{\left(\frac{\beta N}{2}-k\right) !}{\left(\frac{\beta N}{2}\right) !} \quad \text { para } \quad k<\frac{\beta N}{2}+1 .
$$

Esta expresión concuerda con los resultados que se obtuvieron de la distribución para $N=1$ en la Ref. [11]. También, la ecuación (5.101) incluye los resultados conocidos para $\beta=2$ y $N$ arbitraria [36, 40]. Ese resultado es consistente con la distribución [68]

$$
P_{\beta}\left(\tau_{s}\right)=\frac{2 / \beta}{\left(\frac{\beta N}{2}\right) !}\left(\frac{\beta}{2 \tau_{s}}\right)^{2+\beta N / 2} \mathrm{e}^{-\beta / 2 \tau_{s}} .
$$



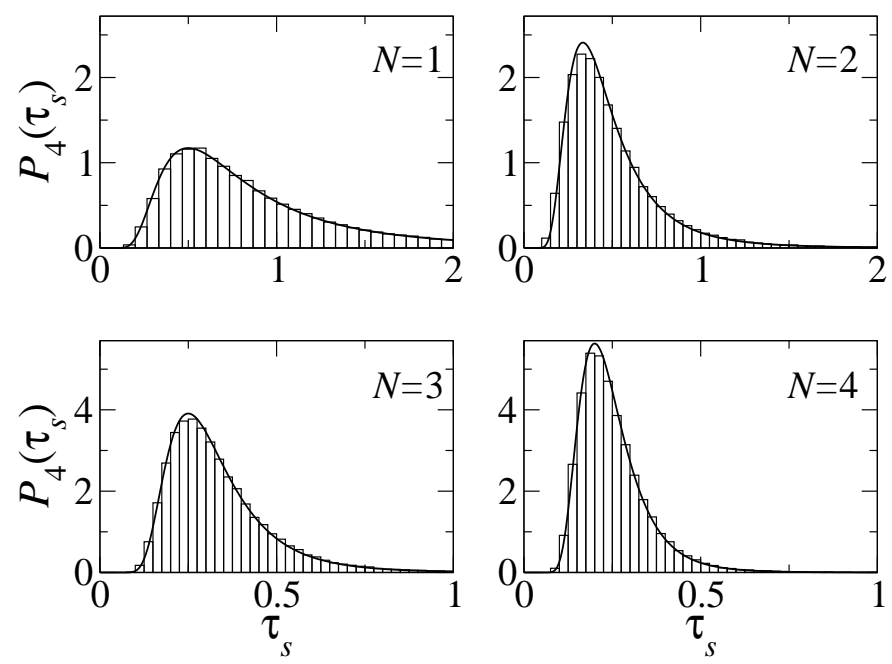

Figura 5.3: Lo mismo que en la figura 5.1 pero para el caso $\beta=4$.

En lo que sigue verificamos esta distribución por medio de simulaciones de teoría de matrices aleatorias.

\subsubsection{Simulaciones numéricas con matrices aleatorias}

El formalismo de Heidelberg de la sección 2.3.2 es el más adecuado para el cálculo de la derivada energética de la matriz de dispersión ya que contiene explícitamente la dependencia en la energía.

Para cada realización diagonalizamos la matriz $S$ para determinar sus valores propios numéricamente. Aquí, sólo nos interesa uno de ellos, por ejemplo $E_{s}(\varepsilon)=\exp \left[\mathrm{i} \theta_{s}(\varepsilon)\right]$, pero evaluado en tres valores de la energía para poder determinar la derivada. Esto es,

$$
\tau_{s}=-\frac{\mathrm{i}}{2 \pi \epsilon} \frac{E_{s}(\epsilon / 2)-E_{s}(-\epsilon / 2)}{E_{s}(0)}
$$

donde $\epsilon=\varepsilon / \Delta$.

En la figura 5.1 comparamos la distribución teórica de la ecuación (5.102), para $\beta=1$, con los resultados numéricos obtenidos de la simulaciones con $10^{5}$ realizaciones de $\tau_{s}$, calculados de acuerdo con la ecuación (5.103), donde se tomó $M=100$ y $\epsilon=0.001$. Observamos buen acuerdo para los casos presentados. Este resultado es importante debido a que no había sido verificado antes. La figura 5.2 muestra en la correspondiente comparación para $\beta=2$, 
la cual está de acuerdo con lo encontrado en la referencia [37].Para $\beta=4$ el resultado teórico muestra un buen ajuste con la simulación numérica como puede observarse en la figura 5.3. Hacemos notar que esta es la primera vez que este caso se ha verificado numéricamente. 


\section{Capítulo 6}

\section{Conclusiones y perspectivas}

En este trabajo de Tesis estudiamos el comportamiento de varias de las propiedades de transporte de ondas en dispositivos mesoscópicos, en distintos situaciones.

Primero, estudiamos el transporte de ondas torsionales en una cavidad elástica unidimensional, en presencia de absorción y procesos directos, desde el punto de vista teórico y experimental. Con ayuda del modelo teórico identificamos las resonancias debidas sólo a las ondas torsionales, que seleccionamos de los resultados experimentales para su análisis. Aquí, encontramos una nueva clase de resonancia de Fano cuyo perfil se debe a una parte resonante que viene de las resonancias torsionales de la cavidad y otra parte debida a los procesos directos. Estos procesos directos son de dos naturalezas diferentes; una de naturaleza torsional, esta es la amplitud continua normal de las resonancias de Fano, y la otra de naturaleza electromagnética que viene de la impedancia de los transductores electromagnético-acústicos que se usaron en la excitación y detección de las ondas en el experimento. El efecto del proceso de medición se tomó en cuenta en la descripción teórica como una suma compleja entre las amplitudes continuas y su interacción con las amplitudes resonantes. Los únicos parámetros relevantes del modelo fueron: la impedancia electromagnética y la matriz de dispersión óptica de las ondas elásticas. Estos se obtuvieron de los datos experimentales, con los cuales encontramos un muy buen acuerdo entre la teoría y el experimento. Aquí, la absorción no afectó de manera significativa al experimento y encontramos que los procesos directos fueron las propiedades más relevantes.

En un segundo problema omitimos la absorción y los procesos directos pero agregamos otro ingrediente: la complejidad que implica el caos. Por medio de una simulación basada en la teoría de matrices aleatorias, repro- 
ducimos tanto la cuantización de la conductancia de un alambre cuántico balístico como las fluctuaciones de ésta con la finalidad de aplicar un análisis similar en un sistema análogo elástico. Así, analizamos las fluctuaciones de la transmisión de ondas flexionales a través de una cavidad con la forma de medio billar de Sinai, obtenidas del experimento; mostramos que la estadística de la transmisión concuerda con las predicciones teóricas, algo que no se había alcanzado antes a pesar de los muchos esfuerzos que se realizaron. Una conclusión importante que obtuvimos es que para sistemas elásticos ni la temperatura ni la absorción rompen la coherencia de fase de las ondas, por lo que los sistema elásticos representan buenos candidatos para corroborar las predicciones del transporte coherente. En los sistemas electrónicos y los de microondas, la absorción y los efectos de la temperatura hacen muy difícil corroborar las predicciones teóricas.

Continuando con la temática de los sistemas caóticos, también estudiamos la caída de voltaje a lo largo de un alambre horizontal con un modo abierto, que consiste de dos alambres balísticos que convergen en un nodo, formando un dispositivo de tres terminales. En este sistema, estudiamos el efecto de las simetrías, tanto en presencia como en ausencia de reversibilidad temporal. Encontramos una clara dependencia de la posición de la sonda usada como punta de medición. Nuestros resultados parecen concordar con los existentes, en presencia de reversibilidad temporal, para alambres desordenados en el límite de régimen localizado. Esto está de acuerdo con nuestra intuición ya que el régimen localizado es el de máximo desorden. Aunque el sistema es caótico, obtuvimos casos en los que se pudo tratar de forma analítica. En un futuro cercano esperamos continuar con el estudio de este sistema para mostrar que, en efecto, el límite de máximo desorden concuerda con el caso caótico. Además, esperamos poder realizar un experimento con el correspondiente análogo elástico que, como hemos visto, ha dado buenos resultados para mostrar la validez del transporte coherente.

También, estudiamos las fluctuaciones de la corriente bombeada en un punto cuántico. Encontramos la magnitud de la corriente bombeada para cualquier número de canales de transmisión, tanto en presencia como ausencia de reversibilidad temporal. Nuestros resultados logran explicar la ausencia de corriente en el caso de pocos canales, reportado en la literatura. Sin embargo, aún es necesario abordar el caso de la simetría simpléctica que no ha sido reportado. Éste será otro objetivo que esperamos abordar pronto.

En la última parte de este trabajo calculamos los momentos generalizados conjuntos de los tiempos propios de retardo para cualquier simetría y un número arbitrario de canales, los cuales son necesarios para cuantificar propiedades de transporte, así como sus fluctuaciones, en sistemas balísticos 
abiertos. Esto se realizó reduciendo los cálculos de los momentos negativos de la distribución generalizada de Laguerre a fórmulas simples que son más fáciles de manejar analíticamente. Como un resultado importante, mostramos que el $k$-ésimo momento de un tiempo propio de retardo difiere de el de un tiempo parcial de retardo, donde la diferencia viene de la repulsión de niveles de los tiempos propios de retardo. De nuestros resultados, también generalizamos la distribución de los tiempos parciales de retardo para cualquier simetría. Aunque consideramos acoplamiento perfecto del sistema con los canales abiertos, nuestros resultados motivan cálculos adicionales para incluir un acoplamiento imperfecto. La distribución de los tiempos de retardo parciales se obtuvieron extrayendo la parte fundamental que viene de la repulsión de niveles. Esta distribución se verificó con simulaciones de teoría de matrices aleatorias para cavidades caóticas balísticas, con acoplamiento ideal para toda clase de simetría. Nuestros resultados reproducen los resultados existentes en la literatura. También proporcionamos la constante de normalización de la distribución de Laguerre. Finalmente, mencionamos que es de interés para la comunidad científica hacer un estudio detallado de estos tiempos propios y parciales, en presencia de absorción y procesos directos. 


\section{Apéndice A}

\section{Conceptos básicos de RMT}

\section{A.1. Ensembles Gaussianos}

En general, obtener el espectro de energías de cavidades caóticas cerradas de manera analítica es muy difícil. Además, aún en los casos en los que se puede calcular de manera numérica, no se obtiene mucha información del análisis individual de los niveles de energía. Es por este motivo que hacer un descripción estadística de este tipo de sistemas es más adecuado. Este análisis tiene la desventaja de que pierde las características particulares del sistema, pero resalta sus propiedades universales.

En RMT, el Hamiltoniano $H$ que representa al sistema bajo estudio se considera como un miembro de un ensemble de matrices aleatorias, es decir

$$
H=\left(\begin{array}{ccc}
H_{11} & \cdots & H_{1 M} \\
\vdots & \ddots & \vdots \\
H_{M 1} & \cdots & H_{M M}
\end{array}\right)
$$

donde los elementos $H_{i j}$ de $H$ son variables aleatorias estadísticamente independientes distribuidas de acuerdo con una ley de probabilidad Gaussiana. En esta teoría, el ensemble que describe al sistema solo está restringido por las propiedades generales de simetría presentes en el sistema los cuales son de tres clases conocidos como ensembles Gaussianos: GOE, GUE y GSE (ensembles Gaussianos ortogonal, unitario y simpléctico, por sus siglas en inglés, respectivamente). Aquí, el promedio sobre la energía se reemplaza por el promedio sobre el ensemble, en donde se hace uso de la propiedad ergódica de los sistemas. 


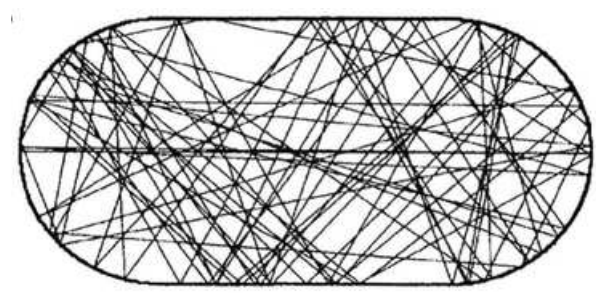

Figura A.1: Ejemplo de una cavidad caótica cerrada. En el interior de la cavidad se observa como debido a la forma de la cavidad, la dinámica clásica de rayos es caótica.

\section{A.1.1. Ensemble ortogonal Gaussiano}

Cuando el sistema presenta invariancia ante inversión en el tiempo (TRI), el Hamiltoniano que describe al sistema es real y simétrico,

$$
H=H^{T},
$$

donde el superíndice $T$ significa la operación transpuesta de $H$. Estas matrices son invariantes bajo transformaciones ortogonales, es decir

$$
H^{\prime}=\mathcal{O}^{T} H \mathcal{O},
$$

donde $\mathcal{O}$ es una matriz ortogonal de tamaño $M \times M\left(\mathcal{O}^{T} \mathcal{O}=\mathcal{O O}^{T}=\mathbf{1}_{M}\right)$ con $\mathbf{1}_{M}$ la matriz unidad de $M \times M$. A un ensemble de matrices $H$ que satisfacen la propiedad (A.3) se le conoce como ensemble ortogonal Gaussiano o GOE. En este caso, los elementos de $H$ son variables aleatorias reales, estadísticamente independientes. En el esquema de Dyson este ensemble se designa como $\beta=1$.

\section{A.1.2. Ensemble unitario Gaussiano}

Para sistemas con ausencia de TRI, el Hamiltoniano que los describe es invariante bajo transformaciones unitarias

$$
H^{\prime}=\mathcal{U}^{\dagger} H \mathcal{U}
$$

donde $\mathcal{U}$ es una matriz unitaria de dimensión $M \times M, \mathcal{U}^{\dagger} \mathcal{U}=\mathcal{U U}^{\dagger}=\mathbf{1}_{M}$, con $\mathcal{U}^{\dagger}$ la transpuesta conjugada de $\mathcal{U}$. Aquí, el Hamiltoniano $H$ del sistema que describe el sistemas es una matriz Hermitiana cuyos elementos son variables aleatorias complejas estadísticamente independientes. A este ensemble se le conoce como ensemble unitario Gaussiano o GUE y corresponde con el caso $\beta=2$ en el esquema de Dyson. 


\section{A.1.3. Ensemble simpléctico Gaussiano}

Si el sistema en consideración tiene simetría de TRI, espín semi-entero y ausencia de simetría de rotación, el Hamiltoniano $H$ que lo describe es invariante bajo transformaciones simplécticas

$$
H^{\prime}=\mathcal{W}^{R} H \mathcal{W}
$$

donde $\mathcal{W}^{R}$ es el dual de la matriz $\mathcal{W}\left(\mathcal{W}^{R} \mathcal{W}=\mathbf{1}\right)$. Así, el Hamiltoniano que representa a un sistema físico con simetría simpléctica es un Hamiltoniano Hermitiano de dimensión $2 M \times 2 M$ y auto-dual ${ }^{1}$; es decir, cumple que

$$
H=H^{\dagger}, \quad H^{R}=H,
$$

cuyos elementos son variables aleatorias estadísticamente independientes. A un ensemble de Hamiltonianos que satisface la propiedad (A.5) se le conoce como ensemble simpléctico Gaussiano o GSE, y se le designa como $\beta=4$ en el esquema de Dyson.

\section{A.2. Ensembles circulares}

El postulado de iguales probabilidades a priori está definido a través del requerimiento de que los ensembles permanezcan invariantes bajo un automorfismo de matrices de dispersión de una clase de simetría dada, $\beta=1$, 2 ó 4 , en el esquema de Dyson. Para $\beta=1$ el automorfismo es

$$
S^{\prime}=U S U^{T}
$$

donde $U$ es una matriz unitaria arbitraria fija; para $\beta=2$ es

$$
S^{\prime}=U S V
$$

donde $V$ es una matriz unitaria arbitraria y fija, independiente de $U$. En ambos casos, requerimos que el elemento de volumen ó medida, $\mathrm{d} \mu(S)$, en el espacio de matrices $S$ permanezca invariante ante dichas transformaciones, dependiendo de la simetría presente en el sistema; es decir

$$
\mathrm{d} \mu^{(\beta)}\left(S^{\prime}\right)=\mathrm{d} \mu^{(\beta)}(S) .
$$

Esta ecuación define los llamados ensembles circulares: COE, CUE y CSE (ensembles circulares ortogonal, unitario y simpléctico, por sus siglas en

\footnotetext{
${ }^{1}$ Una matriz $\mathcal{W}$ se dice que es auto-dual si satisface que $\mathcal{W}^{R}=\mathcal{W}$.
} 
inglés, respectivamente). En el esquema de Dyson estos ensembles corresponden con los casos $\beta=1,2$ y 4, respectivamente. La medida invariante se usa como medida de probabilidad para el ensemble de matrices $S$. Así, la medida invariante representa el caso de un ensemble de matrices de dispersión distribuidas de manera uniforme en el espacio de matrices $S$. Esto es, una distribución uniforme significa que

$$
\mathrm{d} P_{\langle S\rangle}^{(\beta)}(S)=C \mathrm{~d} \mu^{(\beta)}(S),
$$

donde $C$ es una constante de normalización. Es importante notar que un ensemble de matrices de dispersión aleatorias uniformemente distribuidas es tal que el promedio sobre el ensemble es nulo, $\langle S\rangle=0$, que implica ausencia de procesos directos en el sistema.

Para calcular la medida invariante, $\mathrm{d} \mu^{(\beta)}(S)$, escribimos a $S$ en términos de sus variables independientes y calculamos el tensor métrico como sigue. La invariancia sobre la longitud de arco $\mathrm{d} s^{2}$, está dada por

$$
\mathrm{d} s^{2}=\operatorname{Tr}\left(\mathrm{d} S^{\dagger} \mathrm{d} S\right)=\sum_{i, j} g_{i j}(x) \mathrm{d} x_{i} \mathrm{~d} x_{j},
$$

donde $g$ es el tensor métrico y denotamos por $x_{i}$ 's a las variables independientes de $S$, que implica un elemento de volumen invariante

$$
\mathrm{d} \mu^{(\beta)}(S)=\sqrt{\operatorname{det} g} \prod_{i} \mathrm{~d} x_{i} .
$$

Cualquier matriz de dispersión $S$ unitaria se puede parametrizar de la siguiente manera

$$
S=U V
$$

donde $U$ y $V$ son matrices unitarias arbitrarias y fijas de $N \times N$ para $\beta=2$, mientras que $V=U^{T}$ para $\beta=1$, lo más generales posibles. Para $\beta=4$, $V=U^{R}$, donde $U^{R}$ es el dual de la matriz $U$.

La distribución uniforme está dada por medio de la medida invariante definida a través de la relación

$$
\mathrm{d} \mu^{(\beta)}(S)=\mathrm{d} \mu^{(\beta)}\left(U^{\prime} S V^{\prime}\right),
$$

la cual debe estar normalizada, es decir

$$
\int \mathrm{d} \mu^{(\beta)}(S)=1 .
$$

En estos ensembles la matriz $S$ satisface que $\langle S\rangle=0$. 


\section{Apéndice B}

\section{Distribuciones marginales}

La distribución de probabilidad de la matriz $S(f)$ para cada resonancia se puede obtener a partir de la distribución de $S^{\prime}(f)$, que está dada por la ecuación (3.25), por medio de la relación (3.26), con $J\left(S^{\prime}(S)\right)$ el Jacobiano de la transformación que pasa de las coordenadas de $S^{\prime}$ a las coordenadas de $S$; es decir

$$
J\left(S^{\prime}(S)\right)=\left|\frac{\partial\left(R^{\prime}, \theta^{\prime}\right)}{\partial(R, \theta)}\right|=\left(\frac{\partial R^{\prime}}{\partial R}\right)\left(\frac{\partial \theta^{\prime}}{\partial \theta}\right)-\left(\frac{\partial R^{\prime}}{\partial \theta}\right)\left(\frac{\partial \theta^{\prime}}{\partial R}\right) .
$$

Para evaluar el Jacobiano, primero notamos que las matrices $S$ y $S^{\prime}$ están relacionadas por medio de la ecuación (3.22) que se puede escribir en forma explícita como

$$
\sqrt{R^{\prime}} \mathrm{e}^{\mathrm{i} \theta^{\prime}}=\sqrt{R} \mathrm{e}^{\mathrm{i} \theta}-|Z| \mathrm{e}^{\mathrm{i} \phi},
$$

de donde obtenemos las siguientes expresiones

$$
\begin{aligned}
\left(\frac{\partial R^{\prime}}{\partial R}\right) & =1-|Z| R^{-\frac{1}{2}} \cos (\theta-\phi) \\
\left(\frac{\partial R^{\prime}}{\partial \theta}\right) & =2|Z| R^{\frac{1}{2}} \sin (\theta-\phi) \\
\left(\frac{\partial \theta^{\prime}}{\partial R}\right) & =\frac{R^{-\frac{1}{2}} \mathrm{e}^{\mathrm{i} \theta}-R^{\prime-\frac{1}{2}} \mathrm{e}^{\mathrm{i} \theta^{\prime}}\left[1-|Z| R^{-\frac{1}{2}} \cos (\theta-\phi)\right]}{2 \mathrm{i} R^{\prime \frac{1}{2}} \mathrm{e}^{\mathrm{i} \theta^{\prime}}} \\
\left(\frac{\partial \theta^{\prime}}{\partial \theta}\right) & =\frac{\mathrm{i} R^{\frac{1}{2}} \mathrm{e}^{\mathrm{i} \theta}-R^{\prime-\frac{1}{2}} \mathrm{e}^{\mathrm{i} \theta^{\prime}}|Z| R^{\frac{1}{2}} \sin (\theta-\phi)}{\mathrm{i} R^{\prime \frac{1}{2}} \mathrm{e}^{\mathrm{i} \theta^{\prime}}}
\end{aligned}
$$

donde hemos usado que

$$
\left(\frac{\partial R}{\partial \theta}\right)=\left(\frac{\partial \theta}{\partial R}\right)=0
$$


ya que $\theta \neq \theta(R)$. Así, el Jacobiano de la transformación, ecuación (B.1) junto con las ecuaciones (B.3), (B.4), (B.5) y (B.6) conducen a que $J\left(R^{\prime}, \theta^{\prime}\right)=1$. Este resultado era de esperarse ya que en este caso la transformación de coordenadas es sólo la debida a una traslación.

\section{B.1. Distribución marginal radial}

Para obtener la distribución marginal de $R$ integramos la ecuación (3.27) con respecto a $\theta$, es decir

$$
p(R)=\int_{0}^{2 \pi} P(S) \mathrm{d} \theta .
$$

Para resolver esta integral primero notamos que el argumento de la función delta de Dirac en la ecuación (3.27) es una función tanto de $R$ como de $\theta$, ya que $R^{\prime}=R^{\prime}(R, \theta)$, es decir $\delta[F(R, \theta)]$; sin embargo, para el cálculo de las distribuciones marginales una de las variables permanece constante a lo largo del proceso de integración y podemos escribir la función delta en términos de los ceros de su argumento por medio de la siguiente relación conocida

$$
\delta[f(x)]=\sum_{x_{r}} \frac{\delta\left(x-x_{r}\right)}{\left|f^{\prime}(x)\right|_{x=x_{r}}} ; \quad f\left(x_{r}\right)=0,
$$

donde $x_{r}$ son los ceros de la función $f(x)$. Para el caso de la distribución marginal radial, la integral (3.28) es sobre la fase $\theta$ de manera tal que consideramos a $R$ como una constante. Así, el argumento de la función delta se puede escribir como una función sólo de $\theta$ como

$$
f(\theta)=|S-Z|^{2}-R_{0}=\left|\sqrt{R} \mathrm{e}^{\mathrm{i} \theta}-Z\right|^{2}-R_{0},
$$

cuya derivada con respecto a $\theta$ es

$$
\frac{\mathrm{d} f}{\mathrm{~d} \theta}=f^{\prime}(\theta)=2|Z| \sqrt{R} \sin (\theta-\phi) .
$$

Luego, los ceros de $f(\theta)$ son las $\theta_{ \pm}$tales que $f\left(\theta_{ \pm}\right)=0$, es decir

$$
\mathrm{e}^{\mathrm{i} \theta_{ \pm}}=\left[\frac{R+|Z|^{2}-R_{0}}{2|Z| \sqrt{R}} \pm \mathrm{i} \sqrt{1-\left(\frac{R+|Z|^{2}-R_{0}}{2|Z| \sqrt{R}}\right)^{2}}\right] \mathrm{e}^{\mathrm{i} \phi},
$$

de tal manera que la función delta queda como

$$
\delta\left(|S-Z|^{2}-R_{0}\right)=\frac{\delta\left(\theta-\theta_{+}\right)}{\left|f^{\prime}\left(\theta_{+}\right)\right|}+\frac{\delta\left(\theta-\theta_{-}\right)}{\left|f^{\prime}\left(\theta_{-}\right)\right|},
$$


con

$$
\left|f^{\prime}\left(\theta_{ \pm}\right)\right|=\left|\sqrt{\left[R_{0}-(|Z|-\sqrt{R})^{2}\right]\left[(|Z|+\sqrt{R})^{2}-R_{0}\right]}\right|
$$

el cual impone que $|Z|-\sqrt{R} \leq \sqrt{R_{0}} \leq|Z|+\sqrt{R}$, con $R_{0}$ dada por

$$
R_{0}=\left|\sqrt{R} \mathrm{e}^{\mathrm{i} \theta_{ \pm}}-Z\right|^{2} .
$$

Finalmente, de las ecuaciones (3.27), (B.13) y (B.14) en la integral (3.28) nos conducen a la distribución marginal radial de la ecuación (3.29).

\section{B.2. Distribución marginal angular}

De manera similar al caso de la distribución marginal radial, la distribución marginal angular se obtiene mediante la integración de la ecuación (3.27) sobre $R$; es decir

$$
p(\theta)=\int_{0}^{1} P(S) \mathrm{d} R .
$$

En este caso la integral es sobre $R$ por lo que consideramos a $\theta$ como una constante a lo largo del proceso de integración, de manera que ahora tenemos

$$
g(R)=\left|\sqrt{R} \mathrm{e}^{\mathrm{i} \theta}-Z\right|^{2}-R_{0},
$$

cuya derivada es

$$
\frac{\mathrm{d} g}{\mathrm{~d} R}=g^{\prime}(R)=1-\frac{\operatorname{Re}\left(Z^{*} \mathrm{e}^{\mathrm{i} \theta}\right)}{\sqrt{R}} .
$$

Los ceros de la función $g(R)$ son las $R_{ \pm}$tales que $g\left(R_{ \pm}\right)=0$, es decir

$$
\sqrt{R_{ \pm}}=\operatorname{Re}\left(Z^{*} \mathrm{e}^{\mathrm{i} \theta}\right) \pm \sqrt{R_{0}-\left[\operatorname{Im}\left(Z^{*} \mathrm{e}^{\mathrm{i} \theta}\right)\right]^{2}}
$$

en donde tenemos la siguiente restricción

$$
R_{0}-\left[\operatorname{Im}\left(Z^{*} \mathrm{e}^{\mathrm{i} \theta}\right)\right]^{2} \geq 0 .
$$

Esta restricción impone que

$$
\theta \in\left[\phi-\sin ^{-1} \frac{\sqrt{R_{0}}}{|Z|}, \phi+\sin ^{-1} \frac{\sqrt{R_{0}}}{|Z|}\right],
$$


y en este caso $R_{0}$ está dado por

$$
R_{0}=\left|\sqrt{R_{ \pm}} \mathrm{e}^{\mathrm{i} \theta}-Z\right|^{2} .
$$

Así, para el caso angular la ecuación (B.9) se puede escribir como

$$
\delta\left(|S-Z|^{2}-R_{0}\right)=\frac{\delta\left(R-R_{+}\right)}{\left|g^{\prime}\left(R_{+}\right)\right|}+\frac{\delta\left(R-R_{-}\right)}{\left|g^{\prime}\left(R_{-}\right)\right|},
$$

con

$$
g^{\prime}\left(R_{ \pm}\right)=\frac{\sqrt{R_{0}-\left[\operatorname{Im}\left(Z^{*} \mathrm{e}^{\mathrm{i} \theta}\right)\right]^{2}}}{\sqrt{R_{ \pm}}} .
$$

Finalmente, de las ecuaciones (3.27), (B.23) y (B.24) en la integral (3.31) nos conducen a la distribución marginal angular de la ecuación (3.32). 


\section{Apéndice $\mathrm{C}$}

\section{Fluctuaciones del voltaje}

\section{C.1. Matriz $S$ de un dispositivo de tres terminales}

En esta sección derivamos la matriz de dispersión $S$ del dispositivo electrónico de tres terminales de la figura 4.5, ecuación (4.16). El sistema consiste de tres alambres unidimensionales; o guías de onda conectadas a reservorios electrónicos con potenciales químicos $\mu_{1}, \mu_{2}$ y $\mu_{3}$, respectivamente, los cuales convergen en una unión descrita por medio de una matriz unitaria $S_{0}$. En las guías de onda los electrones viajan libremente, mientras que en la región de alguna de las cavidades éstos sufren un proceso de dispersión.

En el caso de un canal, la matriz $S$ del dispositivo de la figura 4.5 es de tamaño $3 \times 3$ que por definición relaciona las amplitudes de las ondas salientes en términos de las correspondientes amplitudes de las ondas entrantes; es decir (ver la figura C.1)

$$
\left(\begin{array}{l}
b_{1} \\
b_{2} \\
b_{3}
\end{array}\right)=S\left(\begin{array}{l}
a_{1} \\
a_{2} \\
a_{3}
\end{array}\right) .
$$

La matriz $S$ total del sistema depende de las matrices de dispersión $S_{j}$ $(j=1,2)$ de cada una de las cavidades, que en términos de sus respectivas amplitudes de las ondas salientes y entrantes están dadas por

$$
\left(\begin{array}{c}
b_{1} \\
a_{1}^{\prime}
\end{array}\right)=S_{1}\left(\begin{array}{c}
a_{1} \\
b_{1}^{\prime}
\end{array}\right), \quad\left(\begin{array}{c}
a_{2}^{\prime} \\
b_{2}
\end{array}\right)=S_{2}\left(\begin{array}{c}
b_{2}^{\prime} \\
a_{2}
\end{array}\right),
$$

así como de la matriz $S_{3}$ que describe a la punta de medición (alambre perfecto)

$$
\left(\begin{array}{l}
a_{3} \\
b_{3}
\end{array}\right)=S_{3}\left(\begin{array}{c}
b_{3} \\
a_{3}
\end{array}\right), \quad \text { con } \quad S_{3}=\left(\begin{array}{ll}
0 & 1 \\
1 & 0
\end{array}\right) .
$$



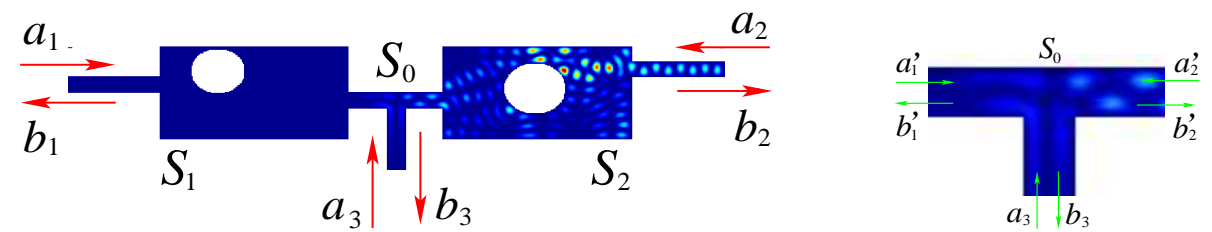

Figura C.1: En la figura de la izquierda se muestran de forma esquemática las ondas entrantes y salientes al dispositivo de tres terminales; mientras que en la figura izquierda se muestran las correspondientes ondas entrantes y salientes en la unión entre los alambres del dispositivo.

Aquí, las matrices $S_{j}$ de $2 \times 2$ son las matrices que describen a cada uno de los alambres, y están dadas por la ecuación (4.19). La matriz $S_{0}$ en la unión está relacionada con las ondas salientes y entrantes de acuerdo a (ver la figura C.1)

$$
\left(\begin{array}{l}
b_{1}^{\prime} \\
b_{2}^{\prime} \\
b_{3}
\end{array}\right)=S_{0}\left(\begin{array}{l}
a_{1}^{\prime} \\
a_{2}^{\prime} \\
a_{3}
\end{array}\right) .
$$

Las ecuaciones (C.2) y (C.3) se pueden escribir en una sola ecuación matricial como

$$
\left(\begin{array}{l}
b_{1} \\
a_{1}^{\prime} \\
a_{2}^{\prime} \\
b_{2} \\
a_{3} \\
b_{3}
\end{array}\right)=\left(\begin{array}{cccccc}
r_{1} & t_{1}^{\prime} & 0 & 0 & 0 & 0 \\
t_{1} & r_{1}^{\prime} & 0 & 0 & 0 & 0 \\
0 & 0 & r_{2} & t_{2}^{\prime} & 0 & 0 \\
0 & 0 & t_{2} & r_{2}^{\prime} & 0 & 0 \\
0 & 0 & 0 & 0 & 0 & 1 \\
0 & 0 & 0 & 0 & 1 & 0
\end{array}\right)\left(\begin{array}{l}
a_{1} \\
b_{1}^{\prime} \\
b_{2}^{\prime} \\
a_{2} \\
b_{3} \\
a_{3}
\end{array}\right),
$$

la cual se puede reescribir de la siguiente manera

$$
\left(\begin{array}{l}
b_{1} \\
b_{2} \\
b_{3} \\
a_{1}^{\prime} \\
a_{2}^{\prime} \\
a_{3}
\end{array}\right)=\left(\begin{array}{cc}
S_{P P} & S_{P Q} \\
S_{Q P} & S_{Q Q}
\end{array}\right)\left(\begin{array}{c}
a_{1} \\
a_{2} \\
a_{3} \\
b_{1}^{\prime} \\
b_{2}^{\prime} \\
b_{3}
\end{array}\right)
$$


donde hemos definido

$$
\left(\begin{array}{cc}
S_{P P} & S_{P Q} \\
S_{Q P} & S_{Q Q}
\end{array}\right)=\mathcal{O}^{T}\left(\begin{array}{cccccc}
r_{1} & t_{1}^{\prime} & 0 & 0 & 0 & 0 \\
t_{1} & r_{1}^{\prime} & 0 & 0 & 0 & 0 \\
0 & 0 & r_{2} & t_{2}^{\prime} & 0 & 0 \\
0 & 0 & t_{2} & r_{2}^{\prime} & 0 & 0 \\
0 & 0 & 0 & 0 & 0 & 1 \\
0 & 0 & 0 & 0 & 1 & 0
\end{array}\right) \mathcal{O}
$$

con $S_{P P}, S_{P Q}, S_{Q P}$ y $S_{Q Q}$ dadas en las ecuaciones (4.17) y (4.18), y la matriz $\mathcal{O}$ es una matriz ortogonal dada por

$$
\mathcal{O}=\left(\begin{array}{llllll}
1 & 0 & 0 & 0 & 0 & 0 \\
0 & 0 & 0 & 1 & 0 & 0 \\
0 & 0 & 0 & 0 & 1 & 0 \\
0 & 1 & 0 & 0 & 0 & 0 \\
0 & 0 & 0 & 0 & 0 & 1 \\
0 & 0 & 1 & 0 & 0 & 0
\end{array}\right)
$$

De la ecuación (C.6) obtenemos una relación para las amplitudes de ondas salientes (con incidencia por la izquierda)

$$
\left(\begin{array}{l}
b_{1} \\
b_{2} \\
b_{3}
\end{array}\right)=S_{P P}\left(\begin{array}{l}
a_{1} \\
a_{2} \\
a_{3}
\end{array}\right)+S_{P Q}\left(\begin{array}{c}
b_{1}^{\prime} \\
b_{2}^{\prime} \\
b_{3}^{\prime}
\end{array}\right) .
$$

y la correspondiente relación para las amplitudes de las ondas entrantes (con incidencia por la derecha)

$$
\left(\begin{array}{l}
a_{1}^{\prime} \\
a_{2}^{\prime} \\
a_{3}
\end{array}\right)=S_{Q P}\left(\begin{array}{l}
a_{1} \\
a_{2} \\
a_{3}
\end{array}\right)+S_{Q Q}\left(\begin{array}{c}
b_{1}^{\prime} \\
b_{2}^{\prime} \\
b_{3}^{\prime}
\end{array}\right) .
$$

Si sustituimos la ecuación (C.4) en (C.10) obtenemos

$$
\left(\begin{array}{c}
a_{1}^{\prime} \\
a_{2}^{\prime} \\
a_{3}
\end{array}\right)=\frac{1}{1-S_{Q Q} S_{0}} S_{Q P}\left(\begin{array}{c}
a_{1} \\
a_{2} \\
a_{3}
\end{array}\right)
$$

y de la ecuación (C.4) en (C.10) obtenemos

$$
\begin{aligned}
\left(\begin{array}{l}
b_{1} \\
b_{2} \\
b_{3}
\end{array}\right) & =S_{P P}\left(\begin{array}{l}
a_{1} \\
a_{2} \\
a_{3}
\end{array}\right)+S_{P Q} S_{0}\left(\begin{array}{l}
a_{1}^{\prime} \\
a_{2}^{\prime} \\
a_{3}
\end{array}\right) \\
& =\left(S_{P P}+S_{P Q} S_{0} \frac{1}{\mathbf{1}-S_{Q Q} S_{0}} S_{Q P}\right)\left(\begin{array}{l}
a_{1} \\
a_{2} \\
a_{3}
\end{array}\right)
\end{aligned}
$$


donde hemos usado la ecuación (C.11). Comparando la ecuación (C.12) con (C.1) obtenemos la matriz $S$ del dispositivo de tres terminales, ecuación (4.16).

\section{C.1.1. Conductor 1 caótico y conductor 2 perfecto con si- metría $\beta=1$}

En el caso cuando el dispositivo presenta simetría $\beta=1$ y el conductor 2 es un alambre perfecto, la función de las fluctuaciones, ecuación (4.23), se reduce a

$$
f_{0}=\frac{\tau_{1}}{1+\sqrt{1-\tau_{1}} \cos \left(2 \psi_{1}\right)}-1,
$$

y de la ecuación (4.26) su distribución está determinada por

$$
p_{1}(f)=\int_{0}^{2 \pi} \int_{0}^{1} \delta\left(f-f_{0}\right) \frac{\mathrm{d} \tau_{1}}{2 \sqrt{\tau_{1}}} \frac{\mathrm{d} \psi_{1}}{2 \pi},
$$

Para resolver ésta integral primero integramos con respecto a la variable $\psi_{1}$, de manera tal que $\tau_{1}$ se puede considerar como una constante, en este primer proceso de integración, y el argumento de la función delta de Dirac sólo dependerá de la variable $\psi_{1}$, es decir $\delta\left[g\left(\psi_{1}\right)\right]$, con $g\left(\psi_{1}\right)=f-f_{0}\left(\tau_{1}, \psi_{1}\right)$. Así, podemos transformar la función delta en la integral (C.14) de acuerdo a la ecuación (B.9). La derivada de $g$ en este caso es

$$
\frac{\mathrm{d} g}{\mathrm{~d} \psi_{1}}=-\frac{2 \tau_{1} \sqrt{1-\tau_{1}} \sin \left(2 \psi_{1}\right)}{\left[1+\sqrt{1-\tau_{1}} \cos \left(2 \psi_{1}\right)\right]^{2}},
$$

y los ceros de $g\left(\psi_{1}\right)$ se pueden escribir en términos de $f$ como

$$
\sqrt{1-\tau_{1}} \sin \left(2 \psi_{1 r_{ \pm}}\right)= \pm \frac{\sqrt{\tau_{1}}}{1+f} \sqrt{(1-f)(1+f)-\tau_{1}},
$$

y la función delta se escribe como

$$
\delta\left[g\left(\psi_{1}\right)\right]=\frac{\delta\left(\psi_{1}-\psi_{1 r_{+}}\right)}{\left|\frac{\mathrm{d} g}{\mathrm{~d} \psi_{1}}\right|_{\psi_{1}=\psi_{1 r_{+}}}}+\frac{\delta\left(\psi_{1}-\psi_{1 r_{-}}\right)}{\left|\frac{\mathrm{d} g}{\mathrm{~d} \psi_{1}}\right|_{\psi_{1}=\psi_{1 r_{-}}}}
$$

De las ecuaciones (C.13), (C.15), (C.16) y (C.17) en la integral (C.14) nos permite obtener el resultado

$$
p_{1}(f)=\frac{1}{\pi} \sqrt{\frac{1-f}{1+f}},
$$

donde $-1<f<1$. 


\section{C.1.2. Conductor 1 caótico y conductor 2 perfecto con si- metría $\beta=2$}

Cuando el dispositivo de tres terminales presenta simetría $\beta=2$, y el alambre 2 es un alambre perfecto, la función de las fluctuaciones, ecuación (4.23), se reduce a

$$
f_{0}=\frac{\tau_{1}}{1+\sqrt{1-\tau_{1}} \cos \left(\psi_{1}+\psi_{1}^{\prime}\right)}-1,
$$

donde de la ecuación (4.26) tenemos que $f_{0}$ se distribuye de acuerdo con

$$
p_{2}(f)=\int_{0}^{2 \pi} \int_{0}^{2 \pi} \int_{0}^{1} \delta\left(f-f_{0}\right) \mathrm{d} \tau_{1} \frac{\mathrm{d} \psi_{1}}{2 \pi} \frac{\mathrm{d} \psi_{1}^{\prime}}{2 \pi} .
$$

De manera similar que en el caso anterior, podemos resolver la integral (4.26) integrando primero con respecto a una de las variables, por ejemplo $\psi_{1}$, esto implica que el resto de las variables $\tau_{1}$ y $\psi_{1}^{\prime}$ se pueden considerar como constantes en esta primera etapa de integración. Así, el argumento $h$ de la función delta se vuelve función sólo de $\psi_{1}$, es decir, $h\left(\psi_{1}\right)=f-f_{0}\left(\psi_{1}\right)$, y su derivada está dada por

$$
\frac{\mathrm{d} h}{\mathrm{~d} \psi_{1}}=-\frac{\tau_{1} \sqrt{1-\tau_{1}} \sin \left(\psi_{1}+\psi_{1}^{\prime}\right)}{\left[1+\sqrt{1-\tau_{1}} \cos \left(\psi_{1}+\psi_{1}^{\prime}\right)\right]^{2}} .
$$

Los ceros de $h\left(\psi_{1}\right)$ se pueden escribir en términos de la ecuación (C.19) como

$$
\sqrt{1-\tau_{1}} \sin \left(\psi_{1 r_{ \pm}}+\psi_{1}^{\prime}\right)= \pm \frac{\sqrt{\tau_{1}}}{1+f} \sqrt{(1-f)(1+f)-\tau_{1}},
$$

y la función delta queda como

$$
\delta\left[h\left(\psi_{1}\right)\right]=\frac{\delta\left(\psi_{1}-\psi_{1 r_{+}}\right)}{\left|\frac{\mathrm{d} h}{\mathrm{~d} \psi_{1}}\right|_{\psi_{1}=\psi_{1 r_{+}}}}+\frac{\delta\left(\psi_{1}-\psi_{1 r_{-}}\right)}{\left|\frac{\mathrm{d} h}{\mathrm{~d} \psi_{1}}\right|_{\psi_{1}=\psi_{1 r_{-}}}} .
$$

De las ecuaciones (C.21), (C.22) y (C.23) en la integral (C.20) obtenemos el resultado

$$
p_{2}(f)=1-f \text {. }
$$




\section{Apéndice D}

\section{Propiedades asociadas a $F_{\theta_{n}}$}

Existen algunas relaciones entre las distribuciones de probabilidad, Ga-

mma, Poisson y Binomial Negativa, que se resumen en las siguientes proposiciones:

Proposición 1. Para $b, b_{1}, b_{2}>0, k, m, n \in \mathbb{Z}_{+}, y x \geq 0$,

$$
\begin{aligned}
& F_{n, b}(x)=\int_{0}^{x} f_{n, b}(t) \mathrm{d} t=\sum_{k=n}^{\infty} P_{\text {Poisson }}(b x, k), \\
& \int_{0}^{x} f_{m, b_{2}}(t) P_{\text {Poisson }}\left(b_{1} t, k\right) \mathrm{d} t=N B_{m, p}(k) F_{m+k, b_{1}+b_{2}}(x),
\end{aligned}
$$

donde $p=b_{2} /\left(b_{1}+b_{2}\right)$ y $P_{\text {Poisson }}(\lambda, k)$ es la distribución de Poisson con parámetro $\lambda$ dada por

$$
P_{\text {Poisson }}(\lambda, k)=\frac{\lambda^{k}}{k !} \mathrm{e}^{-\lambda}, \quad \text { con } \quad k=0,1, \ldots,
$$

y $N B_{m, p}(k)$ es la distribución Binomial Negativa [64] con parámetros $m \in \mathbb{N}$ y $p \in[0,1]$ :

$$
N B_{m, p}(k)=\left(\begin{array}{c}
m-1+k \\
m-1
\end{array}\right)(1-p)^{k} p^{m}, \quad \text { con } \quad k=0,1, \ldots
$$

Demostración. La demostración de (D.1) está basada en la siguiente identidad, la cual está demostrada mediante integración por partes e inducción sobre $n$ (ver el Cap. 4, ejercicio 26, p. 200 de la Ref. [63]),

$$
\frac{1}{n !} \int_{x}^{\infty} t^{n} \mathrm{e}^{-t} \mathrm{~d} t=\sum_{k=0}^{n} \mathrm{e}^{-x} \frac{x^{k}}{k !} .
$$


Por lo tanto, con el cambio de variables $u=b t$ tenemos que

$$
F_{n, b}(x)=1-\frac{1}{(n-1) !} \int_{b x}^{\infty} u^{n-1} \mathrm{e}^{-u} \mathrm{~d} u=\sum_{k=n}^{\infty} \frac{(b x)^{k}}{k !} \mathrm{e}^{-b x}=\sum_{k=n}^{\infty} P_{\text {Poisson }}(b x, k) .
$$

Para demostrar (D.2), sea $p=b_{2} /\left(b_{1}+b_{2}\right)$. Entonces,

$$
\begin{aligned}
\int_{0}^{x} f_{m, b_{2}}(t) P_{\text {Poisson }}\left(b_{1} t, k\right) \mathrm{d} t & =\frac{b_{2}^{m} b_{1}^{k}}{(m-1) ! k !} \int_{0}^{x} t^{m+k-1} \mathrm{e}^{-\left(b_{1}+b_{2}\right) t} \mathrm{~d} t \\
& =N B_{m, p}(k) F_{m+k, b_{1}+b_{2}}(x) .
\end{aligned}
$$

Sea

$$
F_{\theta_{n}}^{(n)}(x):=\int_{\Delta_{n}(x)} \prod_{i=1}^{n} f_{a_{i}, b_{i}}\left(t_{i}\right) \mathrm{d} t_{i},
$$

donde $\Delta_{n}(x)=\left\{\left(t_{1}, \ldots, t_{n}\right): 0 \leq t_{1} \leq t_{2} \leq \cdots \leq t_{n} \leq x\right\}$. Por el principio de Cavallieri, las funciones $F_{\theta_{n}}^{(n)}(x)$ satisfacen la relación de recurrencia

$$
F_{\theta_{n}}^{(n)}(y)=\int_{0}^{y} f_{a_{n}, b_{n}}(x) F_{\theta_{n-1}}^{(n-1)}(x) \mathrm{d} x \text {. }
$$

También, para esta función tenemos las siguientes propiedades:

Proposición 2. Si $n \geq 2$, todas las $a_{i} \in \mathbb{N}, g_{k}:=\sum_{i=1}^{k} b_{i}, y p_{k}:=b_{k} / g_{k}$, entonces

$$
\begin{aligned}
F_{\theta_{1}}^{(1)}(y) & =\sum_{k=a_{1}}^{\infty} P_{\text {Poisson }}\left(b_{1} y, k\right), \quad F_{\theta_{1}}=1, \\
F_{\theta_{n}}^{(n)}(x) & =\sum_{\mathbf{A}^{(n-1)}} \prod_{j=2}^{n} N B_{a_{j}, p_{j}}\left(L_{j-1}\right) F_{a_{n}+L_{n-1}, g_{n}}(x), \\
F_{\theta_{n}} & =\sum_{\mathbf{A}^{(n-1)}} \prod_{j=2}^{n} N B_{a_{j}, p_{j}}\left(L_{j-1}\right),
\end{aligned}
$$

donde $L:=\sum_{i=1}^{n} \ell_{i}$, para $\ell_{i} \in \mathbb{N}, \sum_{\mathbf{A}^{(n-1)}}$ significa suma sobre todas las $\ell_{i} \in \mathbf{A}^{(n-1)}$, con

$$
\mathbf{A}^{(n)}:=\left\{\ell_{1}, \ldots, \ell_{n}: \ell_{i} \geq a_{i}, \ell_{i} \in \mathbb{N}, i=1, \ldots, n\right\} .
$$


Demostración. La primera fórmula en (D.10), es exactamente (D.1) de la Proposición 1. La segunda es clara ya que $F_{\theta_{1}}^{(1)}$ es una función distribución de probabilidad. Para demostrar (D.11), procedemos por inducción sobre $n$. Para $n=2$, (D.11) es una consecuencia directa de la Proposición 1, ya que $a_{1} \in \mathbb{N}$, y el Teorema de la Convergencia Monótona intercambia las series y la integral. Como hipótesis inductiva, supongamos que (D.11) se cumple para $n-1$, con algún $n \geq 3$, e intentaremos demostrarlo para $n$. Con esta hipótesis escribimos la ecuación (D.9) como

$$
\begin{aligned}
F_{\theta_{n}}^{(n)}(y) & =\sum_{\mathbf{A}^{(n-2)}} \prod_{j=2}^{n-1} N B_{a_{j}, p_{j}}\left(L_{j-1}\right) \int_{0}^{y} f_{a_{n}, b_{n}}(x) F_{a_{n-1}+L_{n-2}, g_{n-1}}(x) \mathrm{d} x \\
& =\sum_{\mathbf{A}^{(n-2)}} \prod_{j=2}^{n-1} N B_{a_{j}, p_{j}}\left(L_{j-1}\right) \sum_{k=a_{n-1}+L_{n-2}}^{\infty} N B_{a_{n}, p_{n}}(k) F_{a_{n}+k, g_{n}}(y),
\end{aligned}
$$

donde en la última igualdad usamos primero (D.1) y después (D.2) con $m=a_{n}, k=L_{n-2}, p=p_{n}$, tomando en cuenta que $p_{n}=b_{n} /\left(b_{n}+g_{n-1}\right)$. Si hacemos el cambio de variables $\ell_{n-1}=k-L_{n-2}$, escribimos la última ecuación como

$$
\begin{aligned}
F_{\theta_{n}}^{(n)}(y) & =\sum_{\mathbf{A}^{(n-2)}} \prod_{j=2}^{n-1} N B_{a_{j}, p_{j}}\left(L_{j-1}\right) \\
& \times \sum_{\ell_{n-1}=a_{n-1}}^{\infty} N B_{a_{n}, p_{n}}\left(\ell_{n-1}+L_{n-2}\right) F_{a_{n}+\ell_{n-1}+L_{n-2}, g_{n}}(y) \\
& =\sum_{\mathbf{A}^{(n-1)}} \prod_{j=2}^{n} N B_{j, p_{j}}\left(L_{j-1}\right) F_{a_{n}+L_{n-1}, g_{n}}(y)
\end{aligned}
$$

En el último paso usamos que los conjuntos $\mathbf{A}^{(n)}$ son productos cartesianos [ver (D.13)], de hecho,

$$
\mathbf{A}^{(n-1)}=\mathbf{A}^{(n-2)} \times\left\{a_{n-1}, 1+a_{n-1}, 2+a_{n-1}, \ldots\right\}
$$

Con esto finaliza la demostración de (D.11).

La demostración de (D.12) está basada en el Teorema de la Convergencia Monótona. Esto es,

$$
F_{\theta_{n}}=\lim _{x \rightarrow \infty} F_{\theta_{n}}^{(n)}(x)=\sum_{\mathbf{A}^{(n-1)}} \prod_{j=2}^{n} N B_{a_{j}, p_{j}}\left(L_{j-1}\right) \lim _{x \rightarrow \infty} F_{a_{n}+L_{n-1}, g_{n}}(x)
$$




$$
=\sum_{\mathbf{A}^{(n-1)}} \prod_{j=2}^{n} N B_{a_{j}, p_{j}}\left(L_{j-1}\right)
$$

Observación 3. Para que la Proposición 2 se cumpla no es necesario que las $b_{i}$ 's pertenezcan a $\mathbb{N}$, solo es necesario que sean positivas. 


\section{Bibliografía}

[1] P. A. Mello y N. Kumar, Quantum Transport in Mesoscopic Systems: Complexity and Statistical Fluctuations (Oxford Universtity Press, New York, 2005).

[2] S. Datta, Electronic Transport in Mesoscopic Systems (Cambridge University Press, 1995).

[3] M. Gudiksen, L. J. Lauhon, J. Wang, D. C. Smith y C. M. Lieber, Growth of nanowire superlattice structures for nanoscale photonics and electronics,

Nature 415, 617 (2002).

[4] R. de Picciotto, H. L. Stormer, L. N. Pfeiffer, K. W. Baldwin y K. W. West, Four-terminal resistance of a ballistic quantum wire, Nature 411, 51 (2001).

[5] C. W. J. Beenakker, Random-matrix theory of quantum transport, Rev. Mod. Phys. 69, 731 (1997).

[6] B. L. Al'tshuler, V. E. Kravtsov y I. V. Lerner, Mesoscopic Phenomena in Solids, editado por B. L. Al'tshuler, P. A. Lee y R. A. Webb (Elsevier, Amsterdam, 1991).

[7] Y. Alhassid, Statistical theory of quantum dots, Rev. Mod. Phys. 72, 895 (2000). 
[8] E. Akkermans, G. Montambaux, J.-L. Pichard y Zinn-Justin, Mesoscopic Quantum Physics

(North Holland, Elsevier, Amsterdam, 1995).

[9] M. Martínez-Mares y E. Castaño, Effect of spatial reflection symmetry on the distribution of the parametric conductance derivative in ballistic chaotic cavities, Phys. Rev. E 71, 036201 (2005).

[10] M. Switkes, C. M. Marcus, K. Campman y A. C. Gossard, An Adiabatic Quantum Electron Pump,

Science 283, 1905 (1999).

[11] V. A. Gopar, P. A. Mello y M. Büttiker, Mesoscopic Capacitors: A Statistical Analysis, Phys. Rev. Lett. 77, 3005 (1996).

[12] P. W. Brouwer, K. M. Frahm y C. W. J. Beenakker, Distribution of the quantum mechanical time-delay matrix for a chaotic cavity, Waves Random Media 9, 91 (1999).

[13] H.-D. Gräf, H. L. Harney, H. Lengeler, C. H. Lewenkopf, C. Rangacharyulu, A. Richter, P. Schardt y H. A. Weidenmüller, Distribution of Eigenmodes in a Superconducting Stadium Billiard with Chaotic Dynamics, Phys. Rev. Lett. 69, 1296 (1992).

[14] H.-J. Stöckmann y J. Stein, "Quantum" chaos in billiards studied by microwave absorption, Phys. Rev. Lett. 64, 2215 (1990).

[15] O. Hul, O. Tymoshchuk, S. Bauch, P. M. Koch y L. Sirko, Experimental investigation of Wigner's reaction matrix for irregular graphs with absorption, J. Phys. A: Math. Gen. 38, 10489 (2005).

[16] A. Morales, J. Flores, L. Gutiérrez y R. A. Méndez-Sánchez, Compressional and torsional wave amplitudes in rods with periodic structures, J. Acoust. Soc. Am. 112, 1961 (2002). 
[17] K. Schaadt y A. Kudrolli, Experimental investigation of universal parametric correlators using a vibrating plate,

Phys. Rev. E 60, R3479 (1999).

[18] R. Weaver, Ultrasonics in an aluminum foam, Ultrasonics 36, 435 (1998).

[19] C. Michel, S. Tascu, V. Doya, P. Aschiéri, W. Blanc, O. Legrand y F. Mortessagne, Experimental phase-space-based optical amplification of scar modes, Phys. Rev. E 85, 047201 (2012).

[20] M. Martínez-Mares, Statistical fluctuations of the parametric derivative of the transmission and reflection coefficients in absorbing chaotic cavities, Phys. Rev. E 72, 036202 (2005).

[21] C. M. Marcus, A. J. Rimberg, R. M. Westervelt, P. F. Hopkins y A. C. Gossard, Conductance fluctuations and chaotic scattering in ballistic microstructures,

Phys. Rev. Lett. 69, 506 (1992).

[22] M. Keller, A. Mittal, J. W. Sleight, R. G. Wheeler, D. E. Prober, R. N. Sacks y H. Shtrikmann, Energy-averaged weak localization in chaotic microcavities, Phys. Rev. B 53, R1693 (1996).

[23] I. H. Chan, R. M. Clarke, C. M. Marcus, K. Campman y A. C. Gossard Ballistic Conductance Fluctuations in Shape Space, Phys. Rev. Lett. 74, 3876 (1995).

[24] H. Schanze, H.-J. Stöckmann, M. Martínez-Mares y C. H. Lewenkopf, Universal transport properties of open microwave cavities with and without time-reversal symmetry, Phys. Rev. E 71, 016223 (2005).

[25] H. U. Baranger y P. A. Mello, Mesoscopic Transport through Chaotic Cavities: A Random S-Matrix Theory Approach,

Phys. Rev. Lett. 73, 142 (1994). 
[26] H. U. Baranger y P. A. Mello, Reflection symmetric ballistic microstructures: Quantum transport properties,

Phys. Rev. B 54, 14297 (1996).

[27] G. Báez, M. Martínez-Mares y R. A. Méndez-Sánchez, Absorption strength in absorbing chaotic cavities, Phys. Rev. E 78, 036208 (2008).

[28] R. Schäfer, T. Gorin, T. H. Seligman, and H.-J. Stöckmann, Correlation functions of scattering matrix elements in microwave cavities with strong absorption, J. Phys. A 36, 3289 (2003).

[29] A. M. Martínez-Argüello, R. A. Méndez-Sánchez y M. MartínezMares,

Wave systems with direct processes and localized losses or gains: The nonunitary Poisson kernel,

Phys. Rev. E. 86, 016207 (2012).

[30] M. L. Mehta, Random Matrices, 2nd ed. (Academic Press, San Diego, 1991).

[31] F. J. Dyson, Statistical Theory of the Energy Levels of Complex Systems. I, J. Math. Phys. 3, 140 (1962).

[32] T. Guhr, A. Müller-Groeling, H. A. Weidenmüller, Random-matrix theories in quantum physics: common concepts, Phys. Rep. 229, 189 (1998).

[33] E. P. Wigner, Lower Limit for the Energy Derivative of the Scattering Phase Shift, Phys. Rev. 98, 145 (1955).

[34] F. T. Smith, Lifetime Matrix in Collision Theory, Phys. Rev. 118, 349 (1960).

[35] P. W. Brouwer, K. M. Frahm y C. W. J. Beenakker, Quantum Mechanical Time-Delay Matrix in Chaotic Scattering, Phys. Rev. Lett. 78, 4737 (1997). 
[36] Y. V. Fyodorov y H.-J. Sommers, Statistics of resonance poles, phase shifts and time delays in quantum chaotic scattering: Random matrix approach for systems with broken time-reversal invariance,

J. Math. Phys. 38, 1918 (1997).

[37] P. Šeba, K. Życzkowski y J. Zakrzewski, Statistical properties of random scattering matrices, Phys. Rev. E 54, 2438 (1996).

[38] D. V. Savin, Y. V. Fyodorov y H.-J. Sommers, Reducing nonideal to ideal coupling in random matrix description of chaotic scattering: Application to the time-delay problem, Phys. Rev. E 63, 035202(R) (2001).

[39] Y. V. Fyodorov, D. V. Savin y H.-J. Sommers, Parametric correlations of phase shifts and statistics of time delays in quantum chaotic scattering: Crossover between unitary and orthogonal symmetries,

Phys. Rev. E 55, R4857 (1997).

[40] Y. V. Fyodorov y H.-J. Sommers, Parametric Correlations of Scattering Phase Shifts and Fluctuations of Delay Times in Few-Channel Chaotic Scattering, Phys. Rev. Lett. 76, 4709 (1996).

[41] U. Kuhl, M. Martínez-Mares, R. A. Méndez-Sánchez y H.-J. Stöckmann,

Direct Processes in Chaotic Microwave Cavities in the Presence of Absorption, Phys. Rev. Lett. 94, 144101 (2005).

[42] V. A. Gopar, M. Martínez-Mares y R. A. Méndez-Sánchez, Chaotic scattering with direct processes: a generalization of Poisson's kernel for non-unitary scattering matrices, J. Phys. A: Math. Theor. 41, 015103 (2008).

[43] G. López, P. A. Mello y T. H. Seligman, The Statistical Distribution of the S-Matrix in the One-Channel Case, Zeit. Phys. A 302, 351 (1981).

[44] Marcel Cobián Suárez, "Realización experimental de un sistema elástico infinito a partir de 
uno finito con absorción", Tesis de Maestría (Posgrado en Ciencia e Ingeniería de Materiales, Universidad Autónoma Metropolitana, Unidad Azcapotzalco, 2014).

[45] G. Báez, M. Cobián-Suárez, A. M. Martínez-Argüello, M. MartínezMares y R. A. Méndez-Sánchez,

Scattering of elastic waves in a quasi-one-dimensional cavity: Theory and experiment,

A. Phys. Pol. A 124, 1069 (2013).

[46] A. M. Martínez-Argüello, M. Martínez-Mares, M. Cobián-Suárez, G. Báez y R. A. Méndez-Sánchez, A new Fano resonance in measurement processes, EPL 110, 54003 (2015).

[47] M. Büttiker, Symmetry of electrical conduction, IBM J. Res. Develop. 32, 317 (1988).

[48] P. A. Mello y H. U. Baranger, Interference phenomena in electronic transport through chaotic cavities: an information-theoretic approach, Waves Random Media 9, 111 (1999).

[49] Enrique Flores Olmedo, "Medición del transporte ondulatorio en cavidades caóticas elásticas", Tesis de Doctorado (Posgrado en Ciencia e Ingeniería de Materiales, Universidad Autónoma Metropolitana, Unidad Azcapotzalco, 2016).

[50] A. G. Huibers, S. R. Patel, C. M. Marcus, P. W. Brouwer, C. I. Duruöz y J. S. Harris Jr.,

Distributions of the Conductance and its Parametric Derivatives in Quantum Dots,

Phys. Rev. Lett. 81, 1917 (1998).

[51] E. Flores-Olmedo, A. M. Martínez-Argüello, M. Martínez-Mares, G. Báez, J. A. Franco-Villafañe, and R. A. Méndez-Sánchez, Experimental evidence of coherent transport, Sci. Rep. 6, 25157 (2016).

[52] S. Godoy y P. A. Mello, Random-matrix study of multiprobe mesoscopic devices: A three-probe 
one-dimensional system, Phys. Rev. B 46, 2346 (1992).

[53] F. Barona, A. M. Martínez y M. Martínez, Alambres cuánticos,

Bol. Soc. Mex. Fís. 29, 165 (2015).

[54] A. M. Martínez-Argüello, E. Castaño y M. Martínez-Mares, Random matrix study for a three-terminal chaotic device, AIP Conference Proceedings 1579, 46 (2014).

[55] P. A. Mello, Theory of Random Matrices: Spectral Statistics and Scattering Problems in Mesoscopic Quantum Physics, edited by E. Akkermans, G. Montambaux, J.-L. Pichard, and J.Zinn-Justinm, Elsevier, Amsterdam, 1995.

[56] P. W. Brouwer, Scattering approach to parametric pumping, Phys. Rev. B 58, R10135 (1998).

[57] J. N. H. J. Cremers y P. W. Brouwer, Dephasing in a quantum pump, Phys. Rev. B 65, 115333 (2002).

[58] P. A. Mello, Averages on the unitary group and applications to the problem of disordered conductors, J. Phys. A: Math. Gen. 23, 4021 (1990).

[59] A. M. Martínez-Argüello, M. Martínez-Mares y Julio C. García, Joint moments of proper delay times, J. Math. Phys. 55, 081901 (2014).

[60] I. S. Gradshteyn e I. M. Ryzhik, Table of Integrals, Series and Products, (Academic Press, Inc., Orlando, 1980), p. 1111.

[61] G. Birkhoff y S. Mac Lane, A survey of Modern Algebra, (AKP Classics, U.S.A, 1998). 
[62] I. N. Herstein,

Topics in Algebra,

(John Wiley \& Sons, 2nd Edition, New York, 1975).

[63] S. Ross,

A First Course in Probability,

(Pearson Prentice Hall, 7th Edition, New Jersey, 2006).

[64] Handbook of Mathematical Functions,

Editado por M. Abramowitz e I. A. Stegun,

(Dover Publications, Inc., New York, 1972).

[65] G. Berkolaiko y J. Kuipers, Moments of the Wigner delay times,

J. Phys. A: Math. Theor. 43, 035101 (2010).

[66] F. Mezzadri y N. J. Simm, Moments of the transmission eigenvalues, proper delay times, and random matrix theory. I, J. Math. Phys. 52, 103511 (2011).

[67] M. Martínez-Mares, C. H. Lewenkopf y E. R. Mucciolo, Statistical fluctuations of pumping and rectification currents in quantum dots,

Phys. Rev. B 69, 085301 (2004).

[68] A. M. Martínez-Argüello, A. A. Fernández-Marín y M. MartínezMares,

Delay times in chaotic quantum systems, enviado a EPJ: Special Topics (2016). 


\title{
Scattering of Elastic Waves in a Quasi-One-Dimensional Cavity: Theory and Experiment
}

\author{
G. BÁez, M. Cobián-SuÁrez \\ Universidad Autónoma Metropolitana-Azcapotzalco, A.P. 21-267, 04000 México D.F., Mexico \\ A.M. Martínez-Argüello, M. Martínez-Mares \\ Departamento de Física, Universidad Autónoma Metropolitana-Iztapalapa, A.P. 55-534, 09340 México D.F., Mexico \\ AND R.A. MÉNDEZ-SÁNCHEZ \\ Instituto de Ciencias Físicas, Universidad Nacional Autónoma de México, A.P. 48-3, 62210 Cuernavaca Mor., Mexico
}

\begin{abstract}
We study the scattering of torsional waves through a quasi-one-dimensional cavity both from the experimental and theoretical points of view. The experiment consists of an elastic rod with square cross-section. In order to form a cavity, a notch at a certain distance of one end of the rod was grooved. To absorb the waves, at the other side of the rod, a wedge, covered by an absorbing foam, was machined. In the theoretical description, the scattering matrix $S$ of the torsional waves was obtained. The distribution of $S$ is given by Poisson's kernel. The theoretical predictions show an excellent agreement with the experimental results. This experiment corresponds, in quantum mechanics, to the scattering by a delta potential, in one dimension, located at a certain distance from an impenetrable wall.
\end{abstract}

DOI: $10.12693 /$ APhysPolA.124.1069

PACS: $46.40 . \mathrm{Cd}, 62.30 .+\mathrm{d}, 03.65 . \mathrm{Nk}, 73.21 . \mathrm{Fg}$

\section{Introduction}

The scattering of waves by cavities is a problem of interest in several areas of physics. This is due to the fact that cavities present the majority of phenomena observed in the scattering by complex systems [1-3]. The theoretical and numerical studies of scattering by cavities are extensive including the simplest one-dimensional ones [3-6] and the two-dimensional cavities both, integrable and chaotic [7-11]. The quantum graphs [12] which also display complex behavior can be considered as one-dimensional scattering cavities.

Up to now, scattering experiments by cavities have been performed using mesoscopic cavities [13], quantum corrals [14], microwave cavities [15-22], optical microcavities $[23,24]$ and microwave graphs [25]. In all these experiments the measurements are done in the frequency domain. Wave transport experiments on elastic systems, on the other hand, are scarce and mainly performed in the time domain $[26,27]$. In this paper we introduce a system in which the transport of elastic waves can be studied in the frequency domain from both, the theoretical and experimental points of view.

We organize the paper as follows. In the next section we propose a theoretical model for the scattering of torsional waves by a one-dimensional cavity in an elastic beam. This is done by grooving of a rectangular notch in a specific place of a semi-infinite beam. The scattering matrix $S$ of this system is obtained and we show that its distribution is correctly described by Poisson's kernel.

In Sect. 3 we describe the beam used in the experiment: a notch in one side of the beam and a passive vibration isolation system, on the other side. This beam allows the measurement of the scattering of waves by the cavity formed by the notch and the free-end of the beam. In the same section the experimental setup, used to measure the resonances of the elastic cavity, is also presented. In Sect. 4 we compare the analytical results with the experiment. Some brief conclusions are given in Sect. 5 .

\section{The theoretical model and Poisson's kernel}

In order to study the resonances of an elastic one-dimensional cavity, let us consider a semi-infinite elastic rod with square cross-section of side $W$. As it is shown in Fig. 1, the cavity is formed by a rectangular notch of width $a$ and depth $h$ which have been machined at a distance $L$ from the free-end of the rod. To first order, the torsional wave equation gives a correct description of the scattering of the waves in all regions: inside the cavity, at the notch, and outside the cavity. This model is an analogue of a quantum mechanical delta potential situated at a certain distance from an impenetrable soft wall (Neumann boundary conditions).

The solution of the torsional wave equation, in terms of the wave amplitudes in the different regions of the rod (see Fig. 2), can be written as

$$
\psi(x)= \begin{cases}A_{1} \mathrm{e}^{\mathrm{i} k x}+B_{1} \mathrm{e}^{-\mathrm{i} k x} & \text { for }-L \leq x \leq-a / 2, \\ A_{n} \mathrm{e}^{\mathrm{i} k_{n} x}+B_{n} \mathrm{e}^{-\mathrm{i} k_{n} x} & \text { for }-a / 2 \leq x \leq a / 2, \\ A_{2} \mathrm{e}^{-\mathrm{i} k x}+B_{2} \mathrm{e}^{\mathrm{i} k x} & \text { for } x \geq a / 2,\end{cases}
$$

where the wave number $k_{j}$, in the corresponding region of the beam, is given by [28]:

$$
k_{j}=\frac{2 \pi}{c_{j}} f
$$

with $f-$ the frequency and $c_{j}$ - the velocity of the 


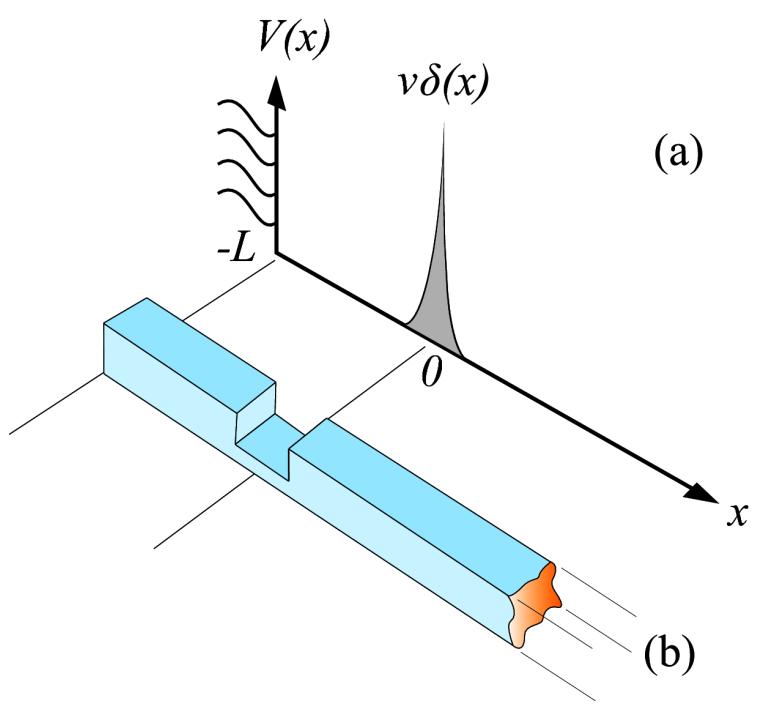

Fig. 1. (a) Quantum scattering cavity formed by a delta potential and an impenetrable barrier. (b) An elastic scattering cavity formed by a notch on a semi-infinite rod.

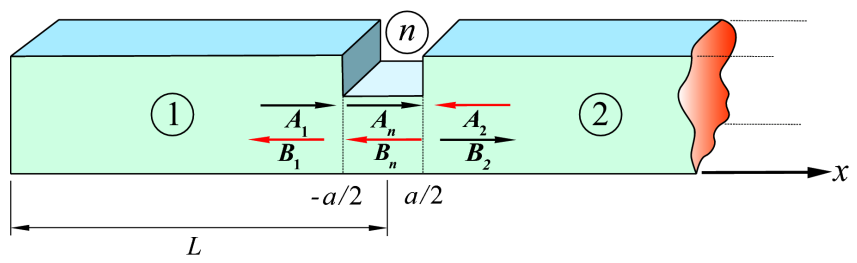

Fig. 2. Waves in the different regions of the semi-infinite rod. The width and depth of the notch are $a$ and $h$, respectively. The length of the cavity is $L-a / 2$.

waves in the respective region. This velocity is related to the shear modulus $G$ and the density $\rho$, through

$$
c_{j}=\sqrt{\frac{G}{\rho} \frac{\alpha_{j}}{I_{j}}},
$$

where $I_{j}$ is the polar momentum of inertia and $\alpha_{j}$ is given by the Navier series in the corresponding region with rectangular cross-section whose base is $W_{j}$ and height $h_{j}$ [for $x \in(-L,-a / 2), h_{1}=W_{1}=W$, while $h_{n}=W-h$ and $W_{n}=W$ for $\left.x \in(-a / 2, a / 2)\right]$; that is,

$$
\begin{aligned}
\alpha_{j} & =\frac{256}{\pi^{6}} \sum_{m=0}^{\infty} \sum_{p=0}^{\infty} \frac{1}{(2 m+1)^{2}(2 p+1)^{2}} \\
& \times \frac{h_{j} W_{j}}{\left(\frac{2 m+1}{h_{j}}\right)^{2}+\left(\frac{2 p+1}{W_{j}}\right)^{2}} .
\end{aligned}
$$

Since the one-dimensional cavity has a free-end at $x=-L$, we impose the condition that $\mathrm{d} \psi(x) / \mathrm{d} x$ vanishes at $x=-L$. We will see below that this boundary condition gives an appropriate description of the experi- ment. The continuity of $\psi(x)$, as well as of the torsional momentum between the different regions, allows us to obtain the scattering matrix associated to the system, namely

$$
S=r_{n}+t_{n} \frac{1}{1-r_{n} \mathrm{e}^{2 \mathrm{i} k(L-a / 2)}} \mathrm{e}^{2 \mathrm{i} k(L-a / 2)} t_{n},
$$

where $r_{n}$ and $t_{n}$ are the reflection and transmission amplitudes through the notch, given by

$$
r_{n}=-\frac{\mathrm{e}^{\mathrm{i} k_{n} a}-\mathrm{e}^{-\mathrm{i} k_{n} a}}{\frac{\lambda-1}{\lambda+1} \mathrm{e}^{\mathrm{i} k_{n} a}-\frac{\lambda+1}{\lambda-1} \mathrm{e}^{-\mathrm{i} k_{n} a}}
$$

and

$$
t_{n}=\frac{\frac{\lambda-1}{\lambda+1}-\frac{\lambda+1}{\lambda-1}}{\frac{\lambda-1}{\lambda+1} \mathrm{e}^{\mathrm{i} k_{n} a}-\frac{\lambda+1}{\lambda-1} \mathrm{e}^{-\mathrm{i} k_{n} a}},
$$

where $\lambda=\alpha_{2} / \alpha_{1}$.

Notice that the scattering matrix depends on the frequency through the wave numbers [see Eq. (2)]. With ideal conditions $S(f)$ is a unitary matrix, such that in the $1 \times 1$ case it becomes a complex number of unit modulus. In a real situation when $S(f)$ is measured in arbitrary units, as it is the case of elastic systems, its modulus is not unit; that is, $S(f)=\sqrt{R_{0}} \mathrm{e}^{\mathrm{i} \theta(f)}$. Therefore, the movement of $S(f)$ as $f$ is varied, describes a circle of radius $\sqrt{R_{0}}$ in the Argand plane; but it does not visit the circle with the same probability; instead $S(f)$ is distributed according to the non-unitary Poisson kernel [5]:

$$
p(\theta)=\frac{1}{2 \pi} \frac{R_{0}-|\bar{S}|^{2}}{|S-\bar{S}|^{2}},
$$

where $\bar{S}$ is the average of $S(f)$ in frequency which, together with $R_{0}$, is obtained from the experiment. Of course, when $R_{0}=1$, Eq. (8) reduces to the ordinary Poisson kernel [29].

For the elastic cavity modeled by Eq. (5), $R_{0}=1$ and $\theta$ depends on the frequency but also on the parameters of the rod which are fixed. As an example, we consider a cavity formed on an aluminum rod of square cross-section of $25.4 \mathrm{~mm}$ of side with a notch of $18.0 \mathrm{~mm}$ depth. The physical parameters of the aluminum alloy 6061-T6, that we use in the experiment, are $G=26 \mathrm{GPa}$ and $\rho=2.7 \mathrm{~g} / \mathrm{cm}^{3}$, such that $\sqrt{G / \rho}=3103.2 \mathrm{~m} / \mathrm{s}$.

The numerical resonances of this cavity, eleven in total, are shown in Fig. 3, where $\theta(f)$ is plotted as a function of $f$ for the frequency range between $14 \mathrm{kHz}$ and $20 \mathrm{kHz}$. Also, in Fig. 3 we show the distribution of $\theta$ in this frequency range, which has been obtained numerically and compared with Poisson's kernel given by Eq. (8) for $R_{0}=1$. The agreement is almost perfect.

\section{The experimental setup}

The theoretical model described in the previous section can be studied experimentally in the corresponding elastic system. As it is seen in Fig. 4, we use an aluminum rod with square cross-section. This rod is divided into four regions: region $\mathrm{I}$ is the quasi-one-dimensional cavity of length $L-a / 2$, where $a$ is the width of a notch of depth $h$, 

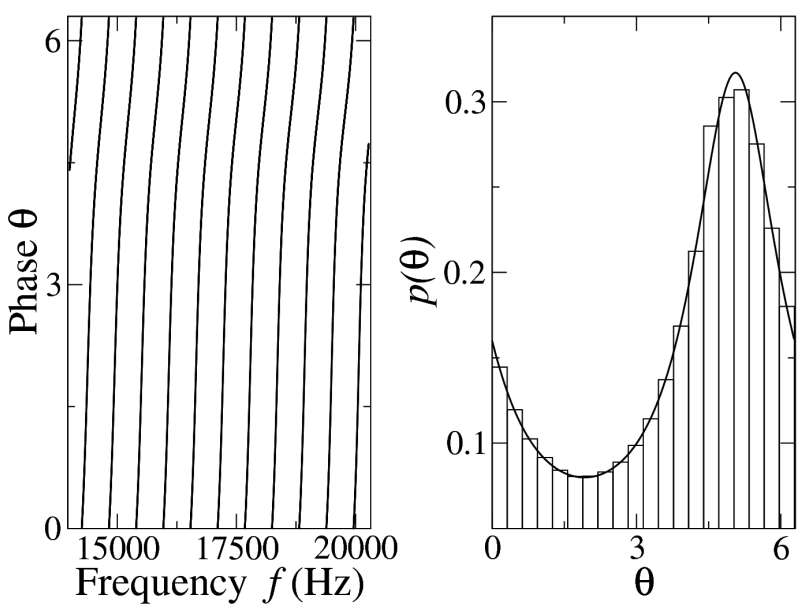

Fig. 3. The phase $\theta$ of the $S$-matrix in the frequency range between $14 \mathrm{kHz}$ and $20 \mathrm{kHz}$ (left part). The distribution of $\theta$, for the same numerical data (histogram), agrees with Poisson's kernel, Eq. (8) (continuous line in the right part).

that defines region II and simulates the quantum delta potential. Regions III and IV mimic the semi-infinite one-dimensional space at the right of the cavity; the vibrations are trapped in a wedge in region IV which acts as a passive attenuation system, together with a polymeric foam that covers it. This scheme minimizes the reflection at the right-end of the rod and consequently the normal modes of the complete system are diminished; it allows to measure the resonances of the cavity formed by the left free-end of the rod and the notch.

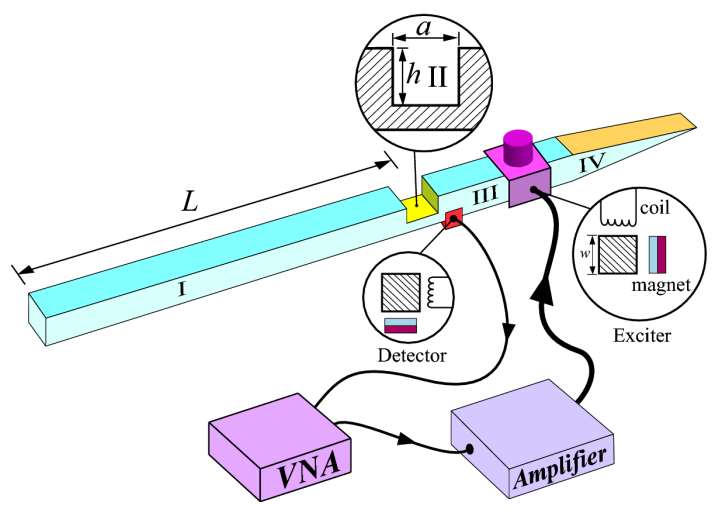

Fig. 4. In the system $L=2.5 \mathrm{~m}$, the depth and width of the notch are $h=0.018 \mathrm{~m}$ and $a=0.0009 \mathrm{~m}$, respectively. The beam has a total length of $3.6 \mathrm{~m}$ and a square cross-section of $0.0254 \mathrm{~m}$ of side. The wedge has a length of $0.40 \mathrm{~m}$ and it is covered by a polymeric foam. The elastic beam is suspended by two nylon strings (not shown). The connection of the equipment is shown at the bottom.

The elastic system is subject to a torsional elastic excitation via an electromagnetic acoustic transducer (EMAT) disposed in torsional wave configuration [30].
The exciter generates a sinusoidal torque at region III of the elastic system. The excitation of the wave is produced by an oscillating magnetic field of frequency $f$ generated by an AC current $I(t)$ on the EMAT's coil, at the same frequency. When a paramagnetic metal is close to the EMAT magnetic field, as Faraday's induction law establishes, eddy currents are produced inside the metal. These currents experience the Lorentz force due to the permanent magnetic field of the EMAT's magnet. In consequence the metal rotates locally in both directions at the frequency $f$.

The response is detected by a second EMAT located outside the cavity, as shown in Fig. 4. As a detector, the EMAT works in the following way: when a rotating metal is located near the field of a permanent magnet, some loops in the metal will have a non-vanishing variable magnetic flux that will produce eddy currents. These currents will generate an oscillating magnetic field that will be measured by the EMAT's coil. In this way, the EMAT detector measures the torsional acceleration of diamagnetic metal [31].

To produce the torsional vibration in the elastic system we use a vector network analyzer (VNA, Anritsu MS-4630B). The VNA produces a sinusoidal signal of frequency $f$, which is amplified by a Cerwin-Vega CV-900 amplifier. The amplified signal is sent to the EMAT exciter. The torsional acceleration measured by the EMAT detector is recorded directly by the VNA for its analysis (see Fig. 4).

\section{Comparison between theory and experiment}

The spectrum of a typical measurement is given in Fig. 5, which shows the observed resonances (thick line). The thin line corresponds to a measurement in which the magnet of the EMAT has been removed; this signal is the impedance curve of the coil and the lines that appear over it are due to radio stations and they must be disregarded. One can observe, also in Fig. 5, that the experimental resonances of the cavity are in very good agreement with the theoretical predictions (vertical marks) of Fig. 3. The comparison between the numerical values of the resonances of torsional waves and the predicted ones, is given in Table, where errors less than $0.1 \%$ are observed. Although some resonances do not appear, they become visible when the location of the EMAT exciter is changed. The remaining resonances belong to other types of vibrations (compressional or flexural).

Within the frequency range measured, between $14 \mathrm{kHz}$ and $20 \mathrm{kHz}$, there are eleven resonances. Due to the impedance of the EMAT's coil, the scattering matrix $S$ describes a circle in the Argand diagram, but displaced from the origin (not shown here).

In Fig. 6 we show two of the resonances, (a) $14819 \mathrm{~Hz}$ and (b) $15960 \mathrm{~Hz}$ (see Table), as they are seen from the center of their corresponding circles. In parts (c) and (d) of Fig. 6 we observe the circles whose radii are not the unit. As we previously explain, this is due to the arbitrary units of measurement in the amplitude. In parts (e) 


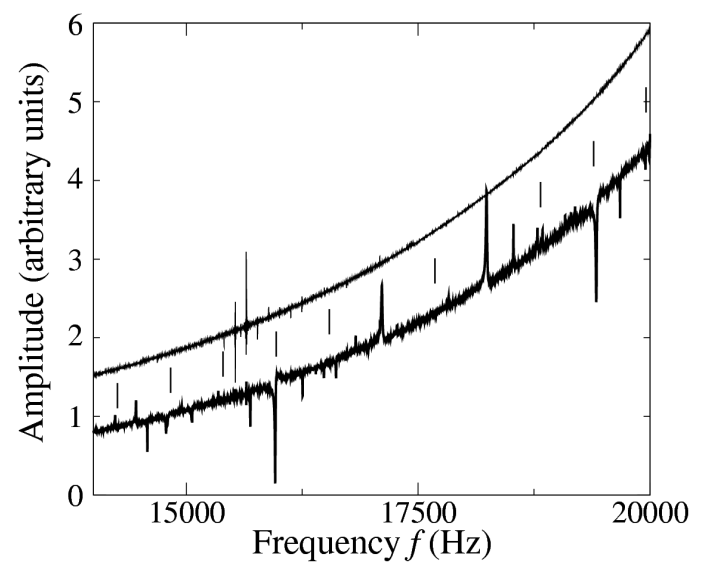

Fig. 5. Spectrum measured with the detector located just outside the cavity after the notch (thick line). The thin line corresponds to the base line measured without the magnet of the EMAT detector. The vertical lines correspond to the theoretical predictions obtained from Fig. 3 (see also Table below).

TABLE

Torsional resonances of a cavity of length $L=2.5 \mathrm{~m}$ formed by one free end and a notch of width $a=0.0009 \mathrm{~m}$ and depth $h=0.018 \mathrm{~m}$. The agreement between the theory and experiment is excellent.

\begin{tabular}{c|c|c}
\hline \hline Resonance & Theory [Hz] & Experiment $[\mathrm{Hz}]$ \\
\hline 1 & 14256 & - \\
2 & 14826 & 14819 \\
3 & 15396 & - \\
4 & 15966 & 15960 \\
5 & 16537 & 16528 \\
6 & 17107 & 17097 \\
7 & 17677 & - \\
8 & 18247 & 18237 \\
9 & 18818 & 18800 \\
10 & 19388 & 19400 \\
11 & 19958 & 19949
\end{tabular}

and (f) we compare the histograms of their phases with the non-unitary Poisson kernel given by Eq. (8), where the values of the average $\bar{S}$ of the $S$-matrix and their radii, taken as averaged quantities, have been extracted from the corresponding experimental data. As can be seen, the agreement is excellent still for the resonance at $f=14819 \mathrm{~Hz}$ that shows the worst agreement.

\section{Conclusions}

We have studied the scattering of torsional waves in a quasi-one-dimensional elastic system. This system consists of a beam with a notch between a free-end and a passive vibration attenuation system that simulates the incoming and outgoing channels at one end of the rod. Theoretically, we obtained the $1 \times 1$ scattering matrix from the solution of the torsional wave equation; the nu-
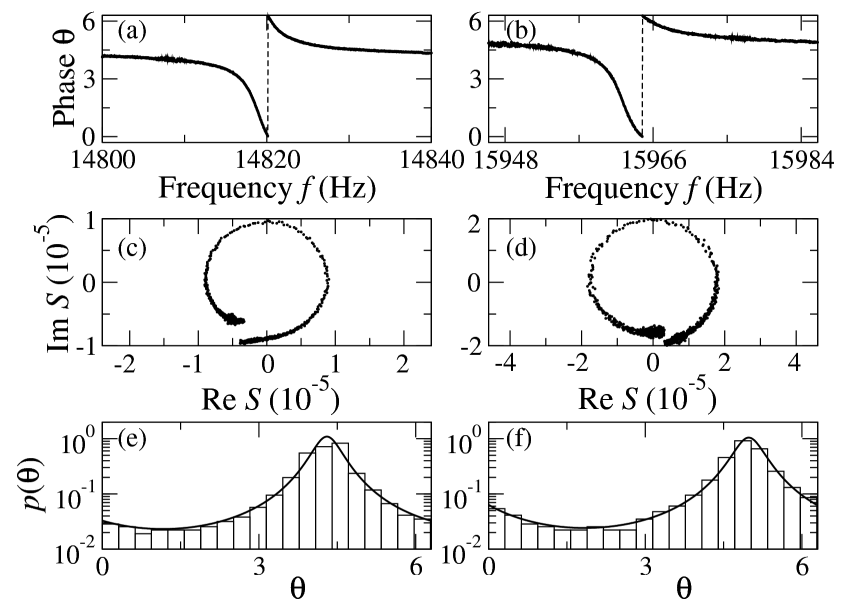

Fig. 6. Phase of the $S$-matrix as a function of frequency $f$ (a) for the resonance at $14819 \mathrm{~Hz}$ and (b) for the resonance at $15960 \mathrm{~Hz}$. The movement of the $S$-matrix as a function of frequency $f$ in the Argand diagram for the same resonances are given in the parts (c) and (d), respectively. The distributions of the phase in parts (e) and (f) show an excellent agreement with the non-unitary Poisson kernel (continuous line). The resonances are observed from the center of the circles (for explanation see the text).

merical predictions helped to select the torsional resonances, among many other vibrational modes that were detected in the experiment. We also verified that the experimental distribution of the phase of the scattering matrix is described by Poisson's kernel, which is a very important theoretical result in scattering of waves by open systems. Despite that the rod is finite we have confirmed that we opened the system from one side, forming in this way a quasi-one-dimensional open cavity.

\section{Acknowledgments}

This work was supported by DGAPA-UNAM under project PAPIIT IN111311. M.C.S. and A.M.M.A. thank financial support from CONACyT. M.M.M. is grateful with the Sistema Nacional de Investigadores and M.A. Torres-Segura for her encouragement.

\section{References}

[1] C.W.J. Beenakker, Rev. Mod. Phys. 69, 731 (1997).

[2] Y. Alhassid, Rev. Mod. Phys. 72, 895 (2000).

[3] P.A. Mello, N. Kumar, Quantum Transport in Mesoscopic Systems: Complexity and Statistical Fluctuations, Oxford University Press, New York 2005.

[4] V.A. Gopar, Ph.D. Thesis, Universidad Nacional Autónoma de México, Distrito Federal 1999.

[5] A.M. Martínez-Argüello, R.A. Méndez-Sánchez, M. Martínez-Mares, Phys. Rev. E 86, 016207 (2012).

[6] V. Domínguez-Rocha, M. Martínez-Mares, J. Phys. A: Math. Theor. 46, 235101 (2013). 
[7] P.A. Mello, H. Baranger, Interference Phenomena in Electronic Transport through Chaotic Cavities: An Information-Theoretic Approach, AIP Conf. Proc. No. 464, AIP, Melville, New York 2008.

[8] G. Báez, M. Martínez-Mares, R.A. Méndez-Sánchez, Phys. Rev. E 78, 036208 (2008).

[9] J.A. Méndez-Bermúdez, G.A. Luna-Acosta, F.M. Izrailev, Physica E 22, 881 (2004).

[10] P.A. Mello, M. Martínez-Mares, Phys. Rev. E 63, 016205 (2000).

[11] P.A. Mello, V.A. Gopar, J.A. Méndez-Bermúdez, in: Quantum scattering and transport in classically chaotic cavities: An overview of past and new results, in Chaotic Systems: Theory and Applications, -206, Eds. C.H. Skiadas, I. Dimotikalis, World Scientific, Singapore 2010, p. 191.

[12] T. Kottos, U. Smilansky, Phys. Rev. Lett. 79, 4794 (1997).

[13] C.M. Marcus, A.J. Rimberg, R.M. Westervelt, P.F. Hopkins, A.C. Gossard, Phys. Rev. Lett. 69 506 (1992)

[14] M.F. Crommie, C.P. Lutz, D.M. Eigler, Nature 363, 524 (1993).

[15] E. Doron, U. Smilansky, A. Frenkel, Phys. Rev. Lett. 65, 3072 (1990).

[16] C.H. Lewenkopf, A. Müller, E. Doron, Phys. Rev. A 45, 2635 (1992).

[17] R.A. Méndez-Sánchez, U. Kuhl, M. Barth, C.H. Lewenkopf, H.-J. Stöckmann, Phys. Rev. Lett. 91, 174102 (2003).

[18] H. Schanze, H.J. Stöckmann, M. Martínez-Mares, C.H. Lewenkopf, Phys. Rev. E 71, 016223 (2005).
[19] U. Kuhl, M. Martínez-Mares, R.A. Méndez-Sánchez, H.-J. Stöckmann, Phys. Rev. Lett. 94, 144101 (2005).

[20] S. Hemmady, X. Zheng, E. Ott, T.M. Antonsen, Jr., S.M. Anlage, Phys. Rev. Lett. 94, 014102 (2005).

[21] D. Laurent, O. Legrand, F. Mortessagne, Phys. Rev. E 74, 046219 (2006).

[22] S. Bittner, B. Dietz, M. Miski-Oglu, P.O. Iriarte, A. Richter, F. Schäfer, Phys. Rev. E 84, 016221 (2011).

[23] J.U. Nöckel, A.D. Stone, Nature 385, 45 (1997).

[24] P. Malara, R. Blanchard, T.S. Mansuripur, A.K. Wojcik, A. Belyanin, K. Fujita, T. Edamura, S. Furuta, M. Yamanishi, P. de Natale, F. Capasso, Appl. Phys. Lett. 102, 141105 (2013).

[25] O. Hul, O. Tymoshchuk, S. Bauch, P.M. Koch, L. Sirko, J. Phys. A, Math. Gen. 38, 10489 (2005).

[26] S. Catheline, N. Benech, J. Brum, C. Negreira, Phys Rev. Lett. 100, 064301 (2008).

[27] A. Morales, A. Díaz-de-Anda, J. Flores, L. Gutiérrez, R.A. Méndez-Sánchez, G. Monsivais, P. Mora, Europhys. Lett. 99, 54002 (2012).

[28] K.F. Graff, Wave Motion in Elastic Solids, Dover, New York 1991.

[29] P.A. Mello, P. Pereyra, T.H. Seligman, Ann. Phys. (New York) 161, 254 (1985).

[30] A. Morales, J. Flores, L. Gutiérrez, R.A. MéndezSánchez, J. Acoust. Soc. Am. 112, 1961 (2002).

[31] A. Morales, L. Gutiérrez, J. Flores, Am. J. Phys. 69,517 (2001). 


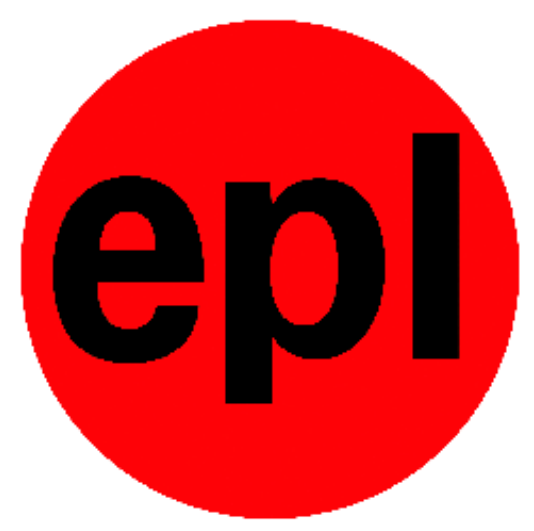

\title{
A LETTERS JOURNAL EXPLORING THE FRONTIERS OF PHYSICS
}

\section{OFFPRINT}

\section{A new Fano resonance in measurement processes}

\author{
A. M. Martínez-Argüello, M. Martínez-Mares, \\ M. Cobián-Suárez, G. Báez and R. A. MÉndez-Sánchez \\ EPL, 110 (2015) 54003
}

Please visit the website

www .epl journal .org

Note that the author(s) has the following rights:

- immediately after publication, to use all or part of the article without revision or modification, including the EPLAformatted version, for personal compilations and use only;

- no sooner than 12 months from the date of first publication, to include the accepted manuscript (all or part), but not the EPLA-formatted version, on institute repositories or third-party websites provided a link to the online EPL abstract or EPL homepage is included.

For complete copyright details see: https://authors.epletters.net/documents/copyright.pdf. 


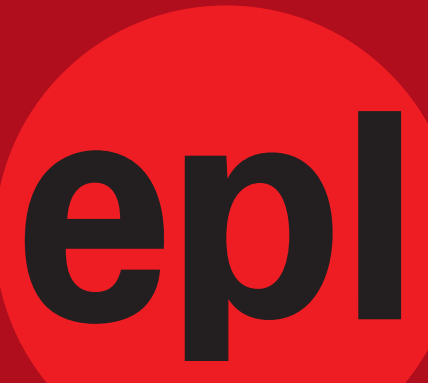

A LetTers JoUrnal EXPLORING

the Frontiers of Physics

\section{AN INVITATION TO SUBMIT YOUR WORK}

www.epljournal.org

\section{The Editorial Board invites you to submit your letters to EPL}

EPL is a leading international journal publishing original, innovative Letters in all areas of physics, ranging from condensed matter topics and interdisciplinary research to astrophysics, geophysics, plasma and fusion sciences, including those with application potential.

The high profile of the journal combined with the excellent scientific quality of the articles ensures that EPL is an essential resource for its worldwide audience. EPL offers authors global visibility and a great opportunity to share their work with others across the whole of the physics community.

\section{Run by active scientists, for scientists}

EPL is reviewed by scientists for scientists, to serve and support the international scientific community. The Editorial Board is a team of active research scientists with an expert understanding of the needs of both authors and researchers.
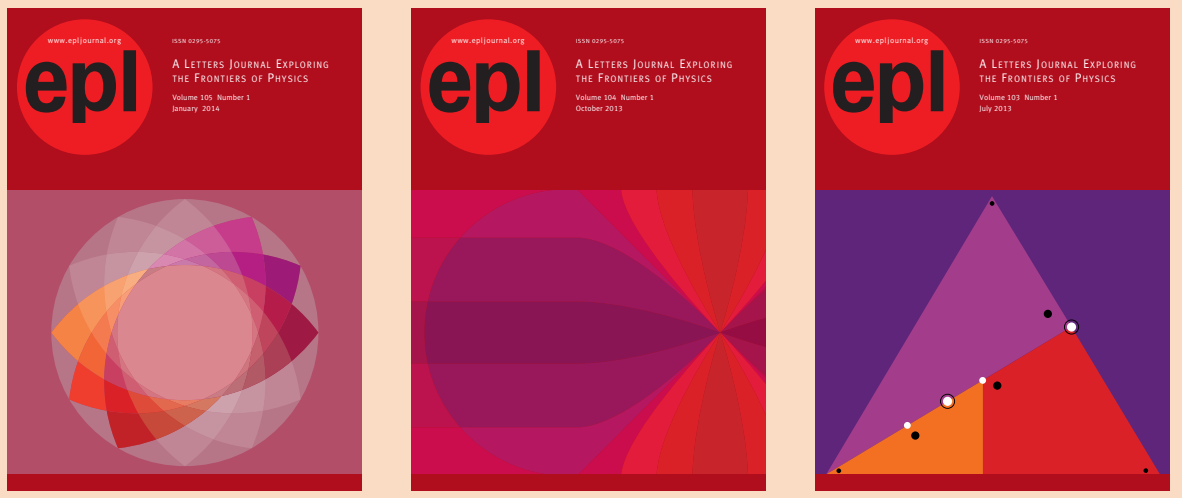


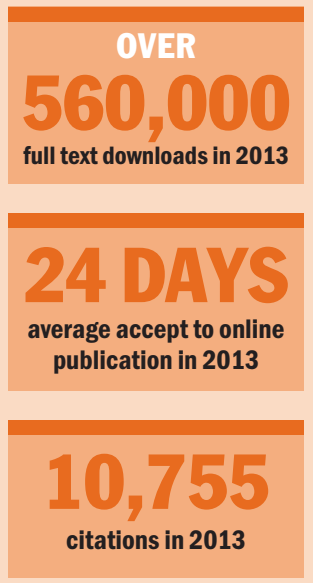

“We greatly appreciate the efficient, professional and rapid processing of our paper by your team."

Cong Lin

Shanghai University

\section{Six good reasons to publish with EPL}

We want to work with you to gain recognition for your research through worldwide visibility and high citations. As an EPL author, you will benefit from:

1

Quality - The 50+ Co-editors, who are experts in their field, oversee the entire peer-review process, from selection of the referees to making all final acceptance decisions.

Convenience - Easy to access compilations of recent articles in specific narrow fields available on the website.

Speed of processing - We aim to provide you with a quick and efficient service; the median time from submission to online publication is under 100 days.

High visibility - Strong promotion and visibility through material available at over 300 events annually, distributed via e-mail, and targeted mailshot newsletters.

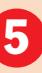

International reach - Over 2600 institutions have access to EPL, enabling your work to be read by your peers in 90 countries.

Open access - Articles are offered open access for a one-off author payment; green open access on all others with a 12-month embargo.

Details on preparing, submitting and tracking the progress of your manuscript from submission to acceptance are available on the EPL submission website www.epletters.net.

If you would like further information about our author service or EPL in general, please visit www.epljournal.org or e-mail us at info@epljournal.org.

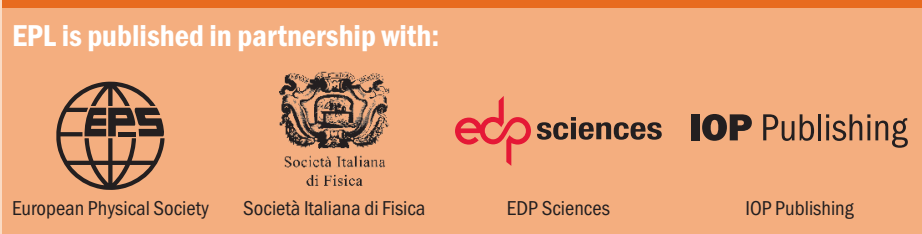

\section{www.epljournal.org}




\title{
A new Fano resonance in measurement processes
}

\author{
A. M. Martínez-Argüello ${ }^{1}$, M. Martínez-Mares ${ }^{1}$, M. Cobián-Suárez $^{2}$, G. Báez ${ }^{2}$ \\ and R. A. MÉNDEZ-SÁNCHEZ ${ }^{3}$ \\ 1 Departamento de Física, Universidad Autónoma Metropolitana-Iztapalapa - Apartado Postal 55-534, \\ 09340 México Distrito Federal, Mexico \\ 2 Departamento de Ciencias Básicas, Universidad Autónoma Metropolitana-Azcapotzalco - Av. San Pablo 180, \\ Col. Reynosa Tamaulipas, 02200 México Distrito Federal, Mexico \\ 3 Instituto de Ciencias Físicas, Universidad Nacional Autónoma de México - Apartado Postal 48-3, \\ 62210 Cuernavaca Mor., Mexico
}

received 29 April 2015; accepted in final form 5 June 2015

published online 25 June 2015

PACS 46.40.Cd - Mechanical wave propagation (including diffraction, scattering, and dispersion)

PACS $62.30 .+d-$ Mechanical and elastic waves; vibrations

PACS 03.65. Nk - Scattering theory

\begin{abstract}
In a wave resonant scattering process the interference of the continuous scattering amplitude with a discrete resonant state, both of the same undulatory nature, gives rise to a Fano resonance profile. We report experimental evidence of a new kind of Fano resonance, in which the continuous amplitude is of a different nature than that of the resonant wave. The continuous amplitude, of a electromagnetic nature, comes from the measurement process and induces a new type of prompt, or rapid, response of the system which we describe theoretically including this response as a direct process.
\end{abstract}

Copyright (c) EPLA, 2015

Introduction. - Fano resonances are ubiquitous in nature. When a resonant scattering processes interferes with the background an asymmetric line-shape, the Fano resonance, profile emerges [1]. The understanding of this interference is an important issue in several areas of physics including nuclear [2,3] and many-body systems [4,5], atomic [6], mesoscopic [7-9], and condensedmatter [10-14] physics, and optics $[15,16]$ (for a recent review see [17]). Therefore, it is expected that Fano resonances appear in the presence of prompt (rapid) responses due to direct processes that occur in the system [18]. Direct processes may take place in experimental measurements where the interaction between the actual system and the equipment may give rise to Fano-type resonances. Among the different ways to perform the measurement of a physical observable, there exist those situations where the equipment enters in direct contact with, or may touch, the system, affecting the measurements in a significant way [19-22]. Besides, there also exist experimental situations in which the equipment does not touch the system at all $[6,23]$. However, there could be another kind of interaction in addition to the existing direct processes in the system that may be relevant. Therefore, it becomes important to distinguish the nature of, and quantify, the prompt responses coming from different kinds of direct processes.
In this letter experimental evidence of two kinds of direct processes is presented. In an elastic system mechanical waves are excited via electromagnetic-acoustic transducers (EMATs) [23-25]. Particular kinds of vibrations can be selectively excited or detected with different configurations of the EMATs. Although the use of EMATs provide the advantage of not being in contact with the system, the electromagnetic field of the exciter enters into the detector inducing an electromagnetic impedance which cannot be ignored [26]. The resonances of the system as a whole, including the EMATs, lie on a base electromagnetic impedance curve because of the prompt response coming from the electromagnetic component, besides the expected prompt response of the scattering of elastic waves. The effect of this impedance can be taken into account in the total prompt response of the system.

Experiment. - The experiment consists of the measurements of the reflected signal for torsional waves by a single port quasi-one-dimensional elastic cavity in a beam. The cavity is formed on a square-cross-section aluminum beam, as shown in fig. 1, between a free end and a notch of width $a$ and depth $h$, grooved at a distance $L$ from the free end. At the other end of the beam a passive vibration isolation system is used $[26,27]$ since 


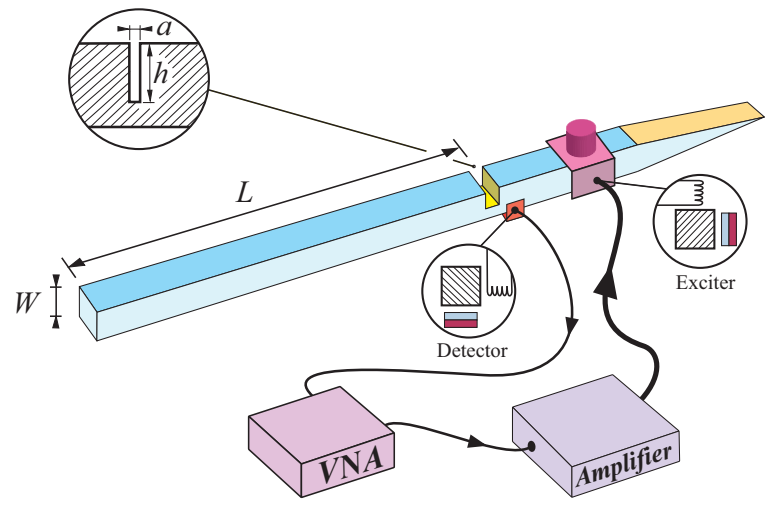

Fig. 1: (Color online) Experimental setup used to form an open elastic wave system (not to scale). A cavity is formed in a beam by machining a notch in it at a certain distance from the free end. The total length of the beam is $3.6 \mathrm{~m}$ with a square crosssection of side $W=25.4 \mathrm{~mm} ; L=2.5 \mathrm{~m}, h=18.0 \mathrm{~mm}$, and $a=0.9 \mathrm{~mm}$. The wedge is $40.0 \mathrm{~cm}$ long. The beam is supported by two nylon threads (not shown). The connection of the equipment is shown at the bottom. The exciter of diameter $\approx 10 \mathrm{~mm}$ is located at $70 \mathrm{~cm}$ from the notch, while the detector is of diameter $\approx 5 \mathrm{~mm}$, and measures the reflected signal with a precision of $3 \mathrm{~mm}$ in the position. An aluminum alloy 6061-T6, with shear modulus $G=26 \mathrm{GPa}$ and density $\rho=2.7 \mathrm{~g} / \mathrm{cm}^{3}$, was used.

single-frequency scattering experiments on elastic systems are difficult to perform due to the formation of stationary patterns [28]. Torsional vibrations are induced using the suitable configuration of the EMAT exciter, which is large enough to produce a detectable torsional acceleration, but it is much smaller than the wavelength; this localization of the excitation does not play a significant role for the one-dimensional propagation [23]. The signal of a vector network analyzer (VNA, Anritsu MS-4630B) is sent to a high-fidelity audio amplifier (Cerwin-Vega CV-900) and then to the EMAT exciter located at a certain distance from the notch, outside the cavity. The torsional acceleration is measured by another EMAT, just outside the cavity, and sent back to the VNA. In this way the nonnormalized $1 \times 1$ scattering matrix,

$$
S(f)=\sqrt{R(f)} \mathrm{e}^{\mathrm{i} \theta(f)},
$$

where $R(f)$ is the reflection coefficient and $\theta(f)$ the phase, is measured.

A typical measurement is shown in fig. 2, in which the magnitude and phase of $S$ are plotted as a function of the frequency $f$, from 14000 to $20500 \mathrm{~Hz}$. According to a numerical simulation based on a simple model of the elastic cavity, eleven torsional resonances can be identified in this range of frequencies [26]; they are indicated with vertical marks in the same figure. The remaining peaks correspond to other modes of vibration or to radio broadcasting stations [26]. It is noticeable that all resonances are located on a base line which comes from the electromagnetic impedance of the system as a whole
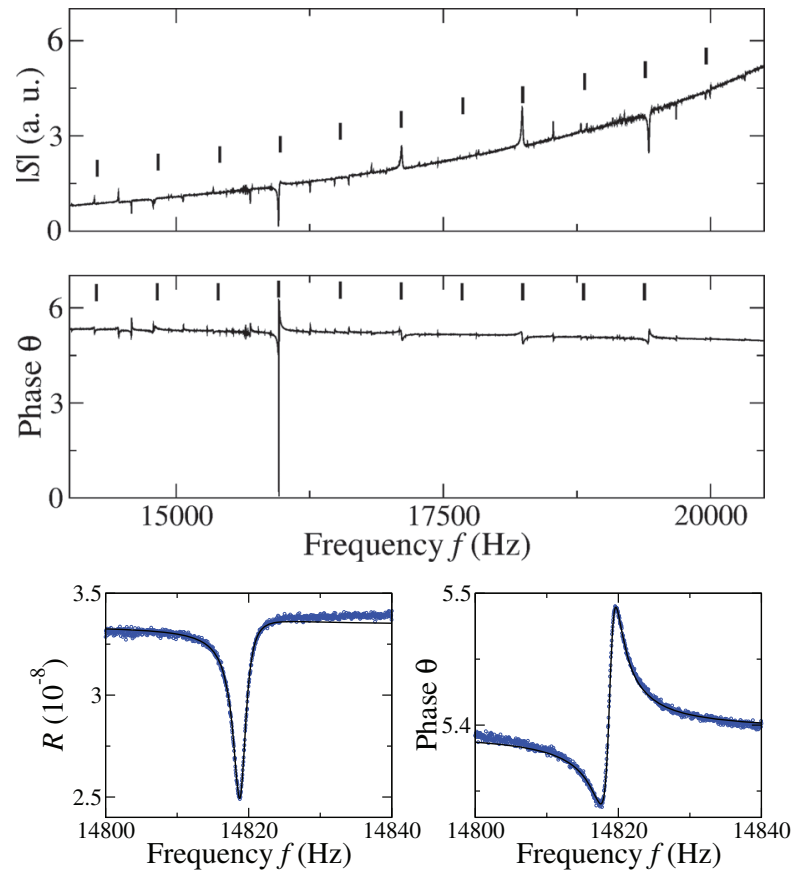

Fig. 2: (Color online) The upper and middle panels show the resonance spectrum for the magnitude and phase of $S(f)$, measured with the detector located just outside the cavity. The vertical marks correspond to theoretical predictions [26]. The lower panels show a fit (continuous black line) of a Fano resonance to the measured resonance at $f_{\text {res }}=14819 \mathrm{~Hz}$.

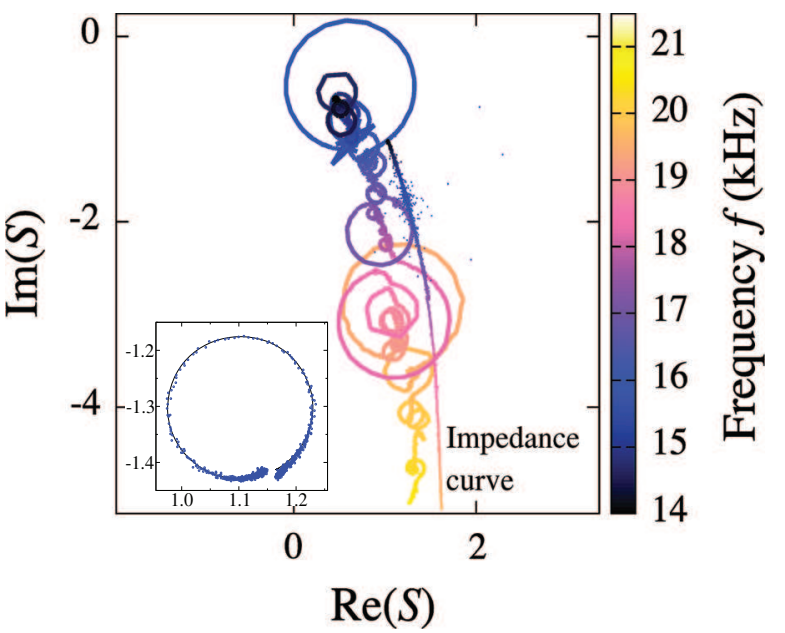

Fig. 3: (Color online) Motion of $S(f)$ in the Argand plane as a function of frequency $f$ for the same resonances, as in fig. 2 . The impedance curve, measured without the elastic system, is given by the thick line. Inset: resonance at $f_{\text {res }}=14819 \mathrm{~Hz}$ compared with eq. (2) in continuous black line.

(see also fig. 3). This was verified by the null hypothesis that corresponds to the measurement without the elastic wave system. In fig. 3 the motion of $S(f)$ in the complex plane is shown as a function of the frequency. Instead of calibrating the scattering matrix as in refs. [22,29], a nonnormalized $S$-matrix will be used and the experimental 
data will be given in arbitrary units. As expected, $S(f)$ describes a circle for each resonance, but its center is not located at the origin. In fact, the circles are superimposed on a base curve, which is parallel to that obtained from the null hypothesis. The circles do not close at all due to the electromagnetic impedance which varies slowly with frequency on each resonance. Because of this, each resonance is analyzed separately. In fig. 3, the result of the null hypothesis is also shown in the Argand plane as the frequency is varied. This displacement is triggered by the impedance giving rise to a Fano-type resonance of the form

$$
S(f)=a+b \frac{2\left(f-f_{\text {res }}\right)-q \Gamma}{2\left(f-f_{\text {res }}\right)-\mathrm{i} \Gamma} .
$$

In the lower panels of fig. 2 we observe that eq. (2) fits very well the measured $S(f)$ for the resonance at $f_{\text {res }}=$ $14819 \mathrm{~Hz}$ of width $\Gamma=2.2 \mathrm{~Hz}$, with $a=(8.08+\mathrm{i} 1.23) \times$ $10^{-5}, b=(-3.45+\mathrm{i} 15.42) \times 10^{-5}$, and the complex Fano parameter $q=0.030+\mathrm{i} 0.842$. In the inset of fig. 3 we observe the same resonance in the Argand plane of $S(f)$ compared with eq. (2).

Comparison between theory and experiment. As has been recently shown in refs. [26,30], the scattering matrix of each resonance, as seen from the center of its own circle, $S^{\prime}(f)$, visits the circumference in a non-uniform way, i.e., according to Poisson's kernel

$$
P^{\prime}\left(S^{\prime}\right)=\frac{1}{2 \pi} \frac{R^{\prime}-\left|\overline{S^{\prime}}\right|^{2}}{\left|S^{\prime}-\overline{S^{\prime}}\right|^{2}} \delta\left(R^{\prime}-R_{0}\right)
$$

where

$$
S^{\prime}(f)=\sqrt{R^{\prime}} \mathrm{e}^{\mathrm{i} \theta^{\prime}(f)}, \quad R^{\prime}=R_{0},
$$

and $R_{0}$ is a constant, experimentally determined as the average of the square of the magnitudes $\left|S^{\prime}(f)\right|$. Here, $\overline{S^{\prime}} \equiv \overline{S^{\prime}(f)}$ is the frequency average of $S^{\prime}(f)$, also determined experimentally. In the jargon of nuclear physics this corresponds to the well-known optical scattering matrix which accounts for the prompt response of the system in the scattering processes [31]. For the theoretical description of the experimental data, as originally measured, the effect of the electromagnetic impedance has to be taken into account.

From the known distribution of $S^{\prime}(f)$, eq. (3), the probability distribution of $S(f)$ for each resonance can be obtained. The measured scattering matrix consists of the scattering matrix of the cavity plus its displacement along the impedance curve and they are both related by a translation,

$$
S(f)=S^{\prime}(f)+Z,
$$

where $Z$ is the position of the center of the circle, as seen from the origin; this displacement is triggered by the impedance, which is different for each resonance. This implies that the optical $S$ matrix, $S_{\text {opt }} \equiv \overline{S(f)}$, consists of two parts: one coming from the scattering process of the system itself and a second coming from the electromagnetic components;

$$
S_{\mathrm{opt}}=\overline{S^{\prime}}+Z
$$

From eq. (3), the probability density distribution of $S(f)$ is given by

$$
P(S)=\frac{1}{2 \pi} \frac{|S-Z|^{2}-\left|S_{\mathrm{opt}}-Z\right|^{2}}{\left|S-S_{\mathrm{opt}}\right|^{2}} \delta\left[R^{\prime}(S)-R_{0}\right],
$$

where $R^{\prime}(S)=|S-Z|^{2}$. In contrast to the ideal case where the impedance is absent and only $S_{\text {opt }}$ is enough to describe the scattering in the system [32,33], this distribution depends on two experimental parameters, $S_{\text {opt }}$ and $Z . \quad R_{0}$ is a constant, not a parameter, whose value deviates from 1 due to the arbitrary units used in the measurements. Since the distributions of $R$ and $\theta$ are not separable, the corresponding marginal distributions will be determined in order to visualize the distribution of $S$.

To obtain the distribution of $R$, eq. (3) is integrated with respect to $\theta$. It is clear that the minimum and maximum values of $\sqrt{R}$ are imposed by $Z$; that is, the minimum (maximum) is given by the sum of the distance to the center $|Z|$, minus (plus) the radius of the circle $\sqrt{R_{0}}$. From eq. (7) we obtain

$$
\begin{aligned}
& p(R)=\frac{1}{2 \pi} \frac{R_{0}-\left|S_{\mathrm{opt}}-Z\right|^{2}}{\sqrt{\left[R-\left(|Z|-\sqrt{R_{0}}\right)^{2}\right]\left[\left(|Z|+\sqrt{R_{0}}\right)^{2}-R\right]}}
\end{aligned}
$$

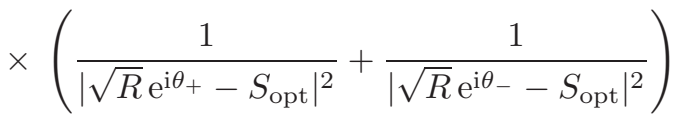

$$
\begin{aligned}
& \times\left[\Theta\left(|Z|+\sqrt{R_{0}}-\sqrt{R}\right)+\Theta\left(\sqrt{R}-|Z|+\sqrt{R_{0}}\right)\right],
\end{aligned}
$$

where $\Theta(z)$ is the Heaviside function of $z$ and

$$
\begin{aligned}
& \sqrt{R} \mathrm{e}^{\mathrm{i} \theta_{ \pm}}=\frac{1}{2 Z^{*}}\left\{\left(R+|Z|^{2}-R_{0}\right)\right. \\
& \left. \pm \mathrm{i} \sqrt{\left[R-\left(|Z|-\sqrt{R_{0}}\right)^{2}\right]\left[\left(|Z|+\sqrt{R_{0}}\right)^{2}-R\right]}\right\} .
\end{aligned}
$$

In a similar way, the marginal phase distribution $q(\theta)$ is obtained by integrating over $R$; the result is

$$
\begin{aligned}
& q(\theta)=\frac{1}{2 \pi} \frac{R_{0}-\left|S_{\mathrm{opt}}-Z\right|^{2}}{\sqrt{R_{0}-\left[\operatorname{Im}\left(Z^{*} \mathrm{e}^{\mathrm{i} \theta}\right)\right]^{2}}} \\
& \times\left(\frac{\sqrt{R_{+}}}{\left|\sqrt{R_{+}} \mathrm{e}^{\mathrm{i} \theta-S_{\mathrm{opt}}}\right|^{2}}+\frac{\sqrt{R_{-}}}{\left|\sqrt{R_{-}} \mathrm{e}^{\mathrm{i} \theta-S_{\mathrm{opt}}}\right|^{2}}\right) \\
& \times\left[\Theta\left(\phi-\sin ^{-1} \frac{\sqrt{R_{0}}}{|Z|}-\theta\right)+\Theta\left(\theta-\phi-\sin ^{-1} \frac{\sqrt{R_{0}}}{|Z|}\right)\right],
\end{aligned}
$$

where $\phi$ is defined through $\mathrm{e}^{\mathrm{i} \phi}=Z /|Z|$;

$$
\sqrt{R_{ \pm}}=\operatorname{Re}\left(Z^{*} \mathrm{e}^{\mathrm{i} \theta}\right) \pm \sqrt{R_{0}-\left[\operatorname{Im}\left(Z^{*} \mathrm{e}^{\mathrm{i} \theta}\right)\right]^{2}}
$$



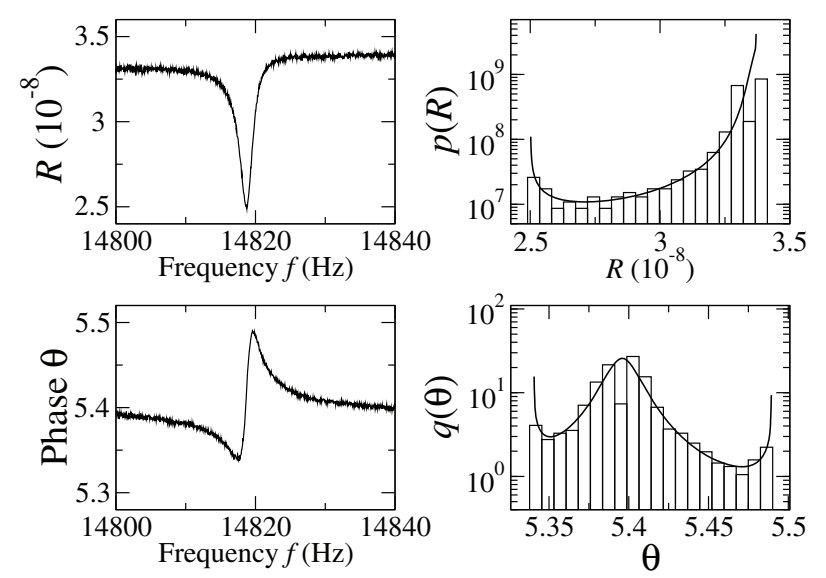

Fig. 4: Resonance at $14819 \mathrm{~Hz}$. In the left panels the squared magnitude and phase of $S(f)$ are shown. The histograms of the experimental data are compared with the theoretical expressions (continuous lines) of eqs. (8) and (10) with $R_{0}=1.6187 \times 10^{-10}, Z=(1.104-\mathrm{i} 1.304) \times 10^{-4}$, and $S_{\text {opt }}=(1.148-\mathrm{i} 1.400) \times 10^{-4}$.
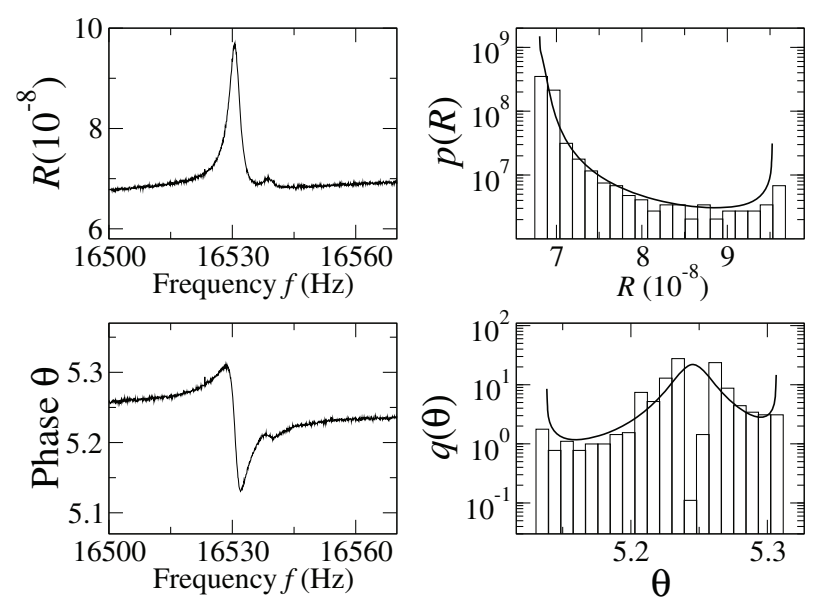

Fig. 5: Resonance at $16530 \mathrm{~Hz}$. Excellent agreement between theory and experiment is found despite the appearance of a second type of resonance, not completely eliminated by the absorber. Here, $R_{0}=5.7327 \times 10^{-10}, Z=(1.390-\mathrm{i} 2.485) \times$ $10^{-4}$, and $S_{\text {opt }}=(1.338-\mathrm{i} 2.289) \times 10^{-4}$.

and $\operatorname{Re}(z)$ and $\operatorname{Im}(z)$ stand for the real and imaginary parts of $z$.

In figs. 4 and 5 the results for two typical experimental resonances, at 14819 and $16530 \mathrm{~Hz}$, are shown. The left panels of both figures show the squared magnitude $(R)$ and phase $(\theta)$ of the amplitude of the measured signal; the effect of the impedance is noticeable. In the right panels the distributions for $R$ and $\theta$ are shown as histograms along with the theoretical expressions (continuous lines) for the marginal distributions, eqs. (8) and (10). The only relevant parameters, $S_{\text {opt }}$ and $Z$, were obtained from the experimental data. Despite the hollow that appears in the histograms of the phase, an excellent agreement is observed. The hollow is due to the lack of data since the experimental resonance does not close completely because of the Fano effect of both kinds of direct processes. In fig. 5 one can observe that another resonance appears around $16540 \mathrm{~Hz}$. The effect of this resonance, that belongs to another type of vibration not completely eliminated by the passive vibration isolation system, does not noticeably affect the distributions.

Conclusions. - In conclusion, a new kind of Fano resonances was found in the scattering of torsional elastic waves in a quasi-one-dimensional cavity. The Fano lineshape is due to 1) a resonant part that comes from the torsional resonances of the cavity and 2) direct processes of two different natures: one of torsional nature, this is the common continuous amplitude in Fano resonances, and the other of electromagnetic nature, coming from the impedance of the electromagnetic-acoustic transducers of the experimental setup. The effect of the measurement processes was taken into account in the theory as a complex sum of the continuous amplitudes and their interaction with the resonant ones. The relevant parameters of the model, the electromagnetic impedance and the optical scattering matrix of the elastic waves, were taken from the experimental data. Excellent agreement between theoretical predictions and experimental results was obtained.

This work was supported by DGAPA-UNAM under project PAPIIT IN103115. MC-S and AMM-A acknowledge the financial support provided by the CONACyT. MM-M is grateful to the Sistema Nacional de Investigadores and M. A. TorREs-SEgurA for her encouragement. The authors also acknowledge the kind hospitality of the Centro Internacional de Ciencias A. C., Mexico, for several group meetings held there.

\section{REFERENCES}

[1] Fano U., Phys. Rev., 124 (1961) 1866.

[2] Adair R. K. et al., Phys. Rev., 76 (1949) 308.

[3] Orrigo S. E. A. et al., Phys. Lett. B, 633 (2006) 469.

[4] Crespi A. et al., Phys. Rev. Lett., 114 (2015) 090201.

[5] Silvestri L. et al., EPL, 109 (2015) 15003.

[6] Отт C. et al., Science, 340 (2013) 716.

[7] Johnson A. C. et al., Phys. Rev. Lett., 93 (2004) 106803.

[8] Bärnthaler A. et al., Phys. Rev. Lett., 105 (2010) 056801.

[9] Gong W. J. et al., EPL, 106 (2014) 30003.

[10] Luk'Yanchuk B. et al., Nat. Mater., 9 (2010) 707.

[11] Rashidian Z. et al., J. Phys.: Condens. Matter, 26 (2014) 255302.

[12] Cortés N. et al., EPL, 108 (2014) 46008.

[13] Faria D. et al., J. Phys.: Condens. Matter, 27 (2015) 175301.

[14] Escudero R. et al., J. Phys.: Condens. Matter, 27 (2015) 015701.

[15] Tribelsky M. I. et al., Phys. Rev. Lett., 100 (2008) 043903.

[16] ZhaO W. and JiAng Y., Opt. Lett., 40 (2015) 93. 
[17] Miroshnichenko A. E. et al., Rev. Mod. Phys., 82 (2010) 2257.

[18] Clerk A. A. et al., Phys. Rev. Lett., 86 (2001) 4636.

[19] Méndez-Sánchez R. A. et al., Phys. Rev. Lett., 91 (2003) 174102.

[20] Kuhl U. et al., Phys. Rev. Lett., 94 (2005) 144101.

[21] Eawniczak M. et al., Phys. Scr., T147 (2012) 014018.

[22] Hemmady S. et al., Phys. Rev. Lett., 94 (2005) 014102.

[23] Morales A. et al., J. Acoust. Soc. Am., 112 (2002) 1961.

[24] Morales A. et al., Am. J. Phys., 69 (2001) 517.
[25] Arreola-Lucas A. et al., J. Sound Vib., 342 (2015) 168.

[26] Báez G. et al., Acta Phys. Pol. A, 124 (2013) 1069.

[27] Xeridat O. et al., Phys. Rev. E, 80 (2009) 035201(R).

[28] Lobkis O. I. et al., Phys. Rev. Lett., 91 (2003) 194101.

[29] Hul O. et al., J. Phys. A: Math. Gen., 38 (2005) 10489.

[30] Martínez-Argüello A. M. et al., Phys. Rev. E, 86 (2012) 016207.

[31] Feshbach H. et al., Phys. Rev., 96 (1954) 448.

[32] López G. et al., Z. Phys. A, 302 (1981) 351.

[33] Mello P. A. et al., Ann. Phys. (N.Y.), 161 (1985) 254. 


\section{SCIENTIFIC REP}

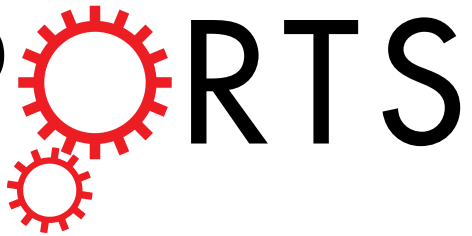

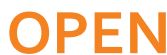

Received: 20 October 2015

Accepted: 04 April 2016

Published: 28 April 2016

\section{Experimental evidence of coherent transport}

\author{
E. Flores-Olmedo ${ }^{1}$, A. M. Martínez-Argüello², M. Martínez-Mares² ${ }^{2}$ G. Báez ${ }^{3}$, \\ J. A. Franco-Villafañe ${ }^{4} \&$ R. A. Méndez-Sánchez ${ }^{4}$
}

Coherent transport phenomena are difficult to observe due to several sources of decoherence. For instance, in the electronic transport through quantum devices the thermal smearing and dephasing, the latter induced by inelastic scattering by phonons or impurities, destroy phase coherence. In other wave systems, the temperature and dephasing may not destroy the coherence and can then be used to observe the underlying wave behaviour of the coherent phenomena. Here, we observe coherent transmission of mechanical waves through a two-dimensional elastic Sinai billiard with two waveguides. The flexural-wave transmission, performed by non-contact means, shows the quantization when a new mode becomes open. These measurements agree with the theoretical predictions of the simplest model highlighting the universal character of the transmission fluctuations.

Decoherence produces drastic effects on the transport properties in both the microscopic and macroscopic domains. It is expected that the electronic transport through clean conductors, ranging from nanometric to micrometric scale, is well described by quantum mechanics at zero temperature. In that situation, the electrons are imagined to propagate freely through the sample keeping phase coherence. Any scattering is triggered by boundaries only, giving rise to quantum interference, which is manifested in the transport properties. For a conductor of irregular shape, or whose shape induces a chaotic dynamic in the limit of ray optics, the conductance shows fluctuations with respect to tuning parameters, such as the magnetic field ${ }^{1}$, Fermi energy ${ }^{2}$, and from sample to sample. These fluctuations are expected to be universal and depend only on symmetry, as coherence is preserved.

However, in experiments at finite temperature, the actual conductance fluctuations are found to be non-universal. Thermal smearing and lattice vibrations, which induce inelastic scattering by phonons, among other sources of dephasing, destroy the phase coherence ${ }^{1,3}$. In this sense, the quantum predictions for the fluctuations of the conductance at zero temperature seem to be inaccessible or at least very difficult to attain at the microscopic scale. Nevertheless, the underlying wave nature of the electrons encourages us to look for undulatory alternatives in which the temperature does not play a significant role. Since the electronic transport can be reduced to a scattering problem, the conductance is proportional to the transmission probability through the sample ${ }^{4}$, classical wave systems are good models to verify the theoretical predictions for coherent transport via the universal fluctuations of the transmission ${ }^{5}$.

The first efforts to demonstrate the universal statistics of transmission through chaotic systems were done in microwave cavities ${ }^{6,7}$. However, the dissipation and the imperfect coupling that occur in these systems ${ }^{8-10}$ diffi- $^{-}$ cult the verification of the predictions for coherent transmission ${ }^{9}$. Only partial and indirect verification has been performed by removing the imperfect coupling effects through a normalization procedure ${ }^{11}$. These difficulties can be handled in mechanical wave systems ${ }^{12}$, since it has been found that the quality factor in these systems is sometimes orders of magnitude higher ${ }^{13}$ than those found in superconducting microwave cavities ${ }^{14}$, thus not affecting phase coherence ${ }^{15}$.

Here we report on the realization of a two-dimensional elastic cavity with the shape of a non-symmetric, half Sinai billiard, with two waveguides which allows us to measure coherent transmission, as illustrated in Fig. 1. The elastic cavity is constructed on an aluminum plate of thickness $h$ and is open through the different modes

${ }^{1}$ Posgrado en Ciencias e Ingeniería, División de Ciencias Básicas e Ingeniería, Universidad Autonóma MetropolitanaAzcapotzalco. Av. San Pablo 180, Col. Reynosa Tamaulipas, 02200 México DF, Mexico. ${ }^{2}$ Departamento de Física, Universidad Autónoma Metropolitana-Iztapalapa, Apartado Postal 55-534, 09340 México Distrito Federal, Mexico. ${ }^{3}$ Departamento de Ciencias Básicas, Universidad Autónoma Metropolitana-Azcapotzalco, Av. San Pablo 180, Col. Reynosa Tamaulipas, 02200 México Distrito Federal, Mexico. ${ }^{4}$ Instituto de Ciencias Físicas, Universidad Nacional Autónoma de México, Apartado Postal 48-3, 62210 Cuernavaca Mor., Mexico. Correspondence and requests for materials should be addressed to R.A.M.-S. (email: mendez@icf.unam.mx) 

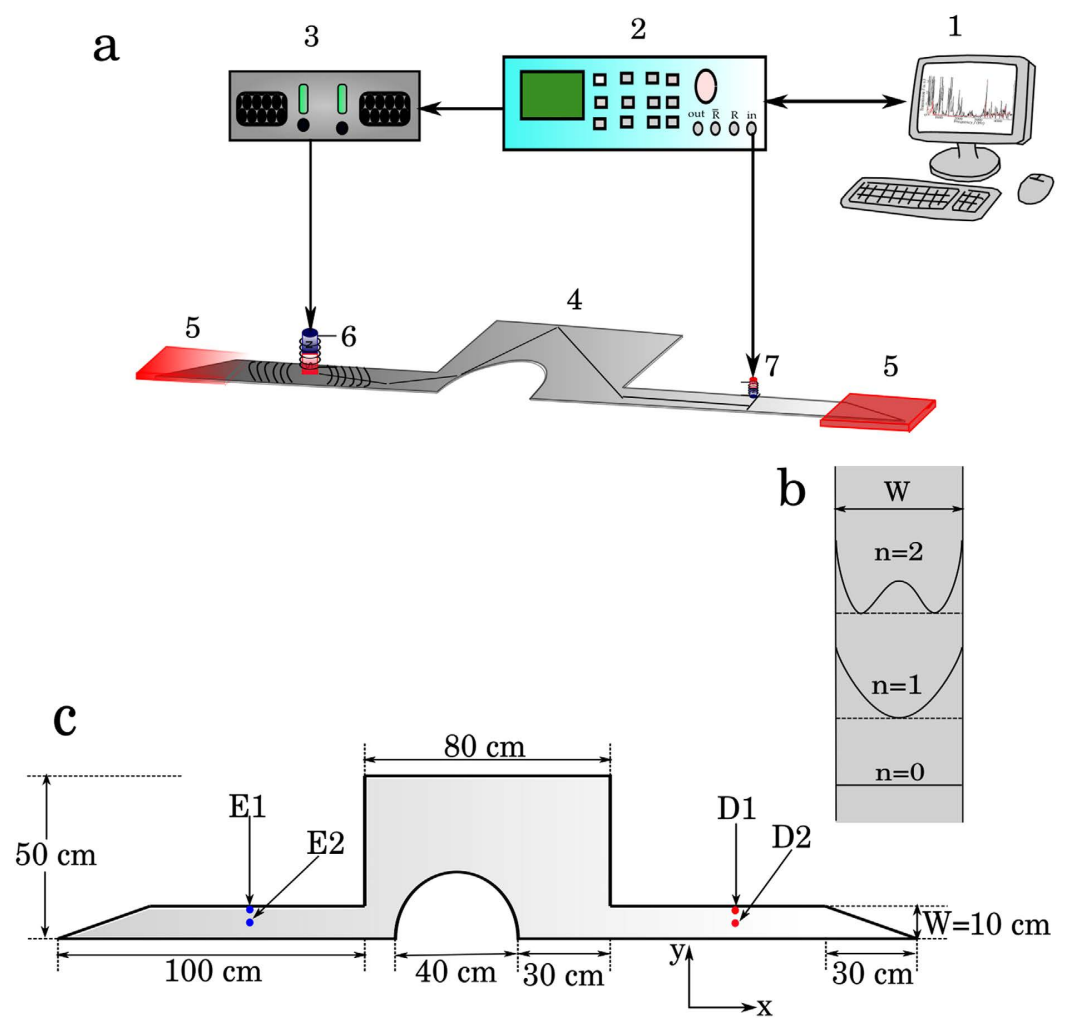

Figure 1. Experimental setup. (a) Workstation (1), vector network analyzer (2), high-fidelity audio amplifier (3), aluminum plate (4), passive vibration isolation systems (5), electromagnetic-acoustic transducers, exciter (6) and detector (7). (b) Schematic drawing of the intensity of the lower flexural channels of a thin elastic waveguide. (c) Mechanical Sinai billiard on the aluminum plate of thickness $h=6.35 \mathrm{~mm}$ (not shown); the waveguides are of width $W=10 \mathrm{~cm}$ and the wedges at the end of them are covered by foams to avoid unwanted reflections. The points E1 and E2 (D1 and D2), indicate the used locations of the exciter (detector). Mechanical properties of the aluminum plate: the Young modulus, density, and Poisson's ratio are $E=71.1 \mathrm{GPa}$, $\rho=2,708 \mathrm{~kg} / \mathrm{m}^{3}$, and $\nu=0.36$, respectively.

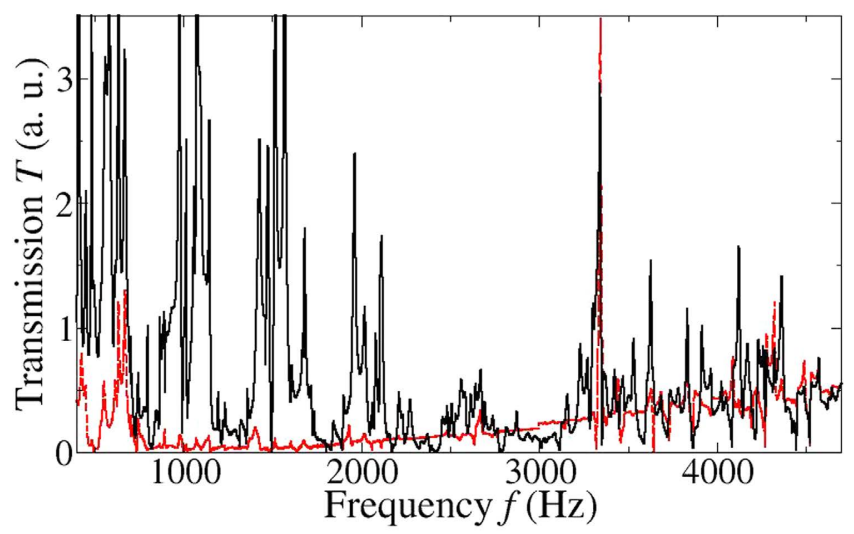

Figure 2. Transmission spectrum. The transmission probability $T$, in arbitrary units (a.u.), is measured at D1 (black line) and D2 (red line) when the excitation is at E2. Below $3,300 \mathrm{~Hz}$ only the mode $n=1$ is transmitted despite of both modes, $n=0$ and $n=1$, are open. Above $3,300 \mathrm{~Hz}$ a different behavior is observed, which corresponds to the opening of the mode with $n=2$.

(or channels) supported by the two waveguides of width $W$. Flexural vibrations are excited and detected using non-contact electromagnetic-acoustic transducers ${ }^{16}$. Direct transmission between both waveguides, one in front of each other, is diminished by locating the semicircle of the Sinai billiard as an obstacle between them. To prevent 

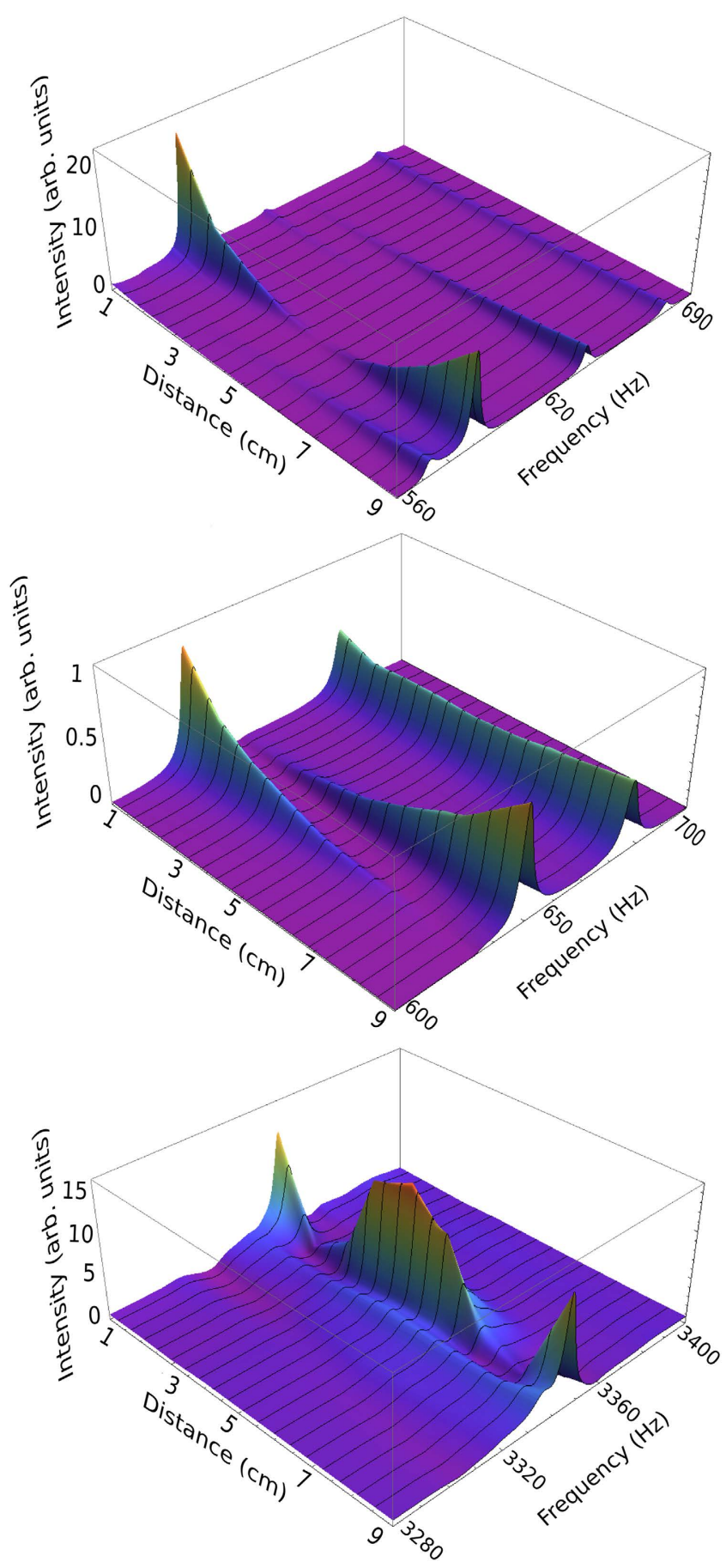

Figure 3. Measured modes. Profiles of the transmitting flexural modes. In the upper panel a mode with $n=1$ is observed at $580 \mathrm{~Hz}$. The middle panel shows a mode with $n=0$ close to $676.8 \mathrm{~Hz}$. Both modes are present below $3,300 \mathrm{~Hz}$. A mode with $n=2$ is observed at $3,360 \mathrm{~Hz}$ (lower panel). At $640 \mathrm{~Hz}$ and $660 \mathrm{~Hz}$ mixtures of modes with $n=0$ and $n=1$ appear; they show a very small amplitude. The transmission intensities were measured across the width of the waveguide.

wave reflections at the end of the waveguides, passive vibration isolation systems are used; these consist of wedges covered by foam pads.

The experimental setup consists of a workstation that controls a vector network analyzer (VNA) through a GPIB interface (see Fig. 1a). The VNA, Anritsu Model MS4630B, performs an up-chirp within the audible frequency range; a Cerwin-Vega high-fidelity audio amplifier CV-900 intensifies the signal coming from the VNA and sends it to an electromagnetic-acoustic transducer (EMAT). The EMAT, which consists of a coil and a permanent magnet, is located at one lead of the billiard. It excites flexural waves when the dipole moment axis of both the EMAT's coil and permanent magnet coincide with the normal to the plate ${ }^{17}$. The flexural waves travel 

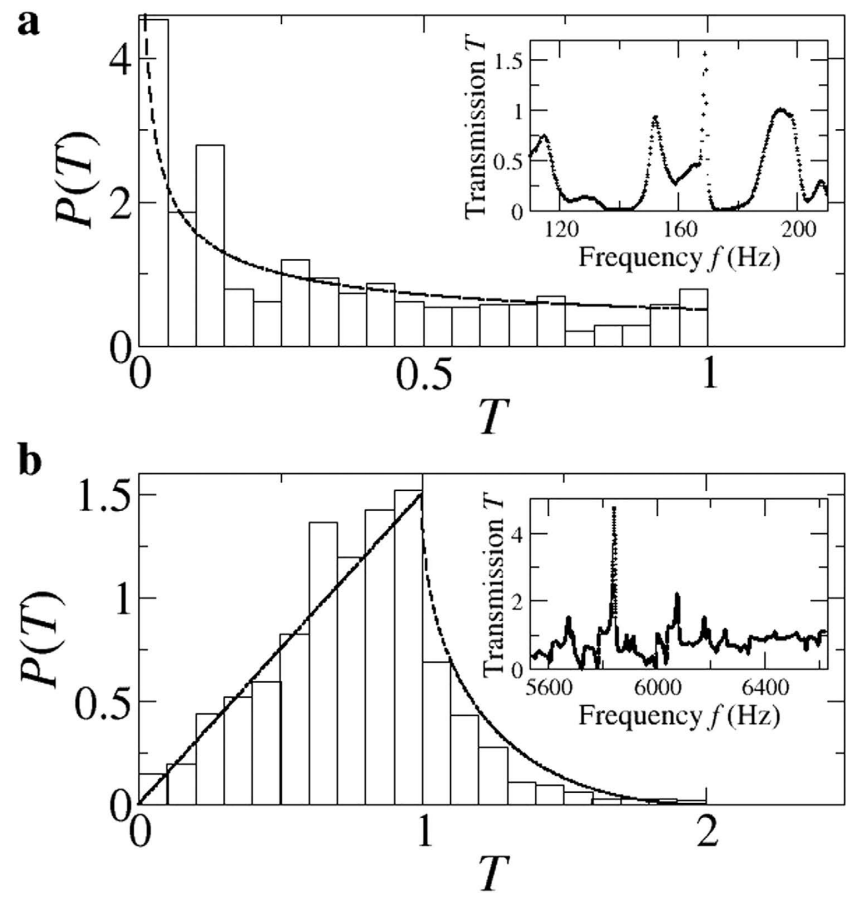

Figure 4. Transmission distributions. Experimental distributions for the flexural transmission probability $T$ (histograms) show excellent agreement with the predictions of the simplest model of random matrix theory for quantum transport (dashed lines). (a) One-channel case. (b) Two-channels case. Insets: Transmission spectra normalized to the theoretical average in the corresponding frequency window. The histograms do not take into account the high and thin resonances appearing in the insets since they correspond to in-plane modes.

along the elastic waveguide and enter into the thin-plate mechanical billiard, where they are scattered. A second EMAT of smaller dimensions, with the same configuration of coil and permanent magnet as the exciter, located at the opposite waveguide, is used to detect the flexural transmission. The signal measured by the EMAT detector is sent back to the VNA. The passive vibration isolation systems yield very good absorption for frequencies higher than $250 \mathrm{~Hz}$, avoiding in this way undesirable stationary patterns. The low frequency motion for flexural waves in an elastic waveguide with free boundary conditions, is quantized into transverse modes as the wave propagates along the waveguide. The first three modes are sketched in Fig. 1b. In Fig. 1c the dimensions of the billiard and waveguides are depicted, where the points indicate the positions of the exciter (E) on one side of the cavity and the detector (D) on the opposite side. The four possible exciter-detector configurations are: E1-D1, E1-D2, E2-D1, and E2-D2, where E1 and D1 are $1 \mathrm{~cm}$ far from the edge of the waveguide, and E2 and D2 are $5 \mathrm{~cm}$ far from the edge. These positions are $40 \mathrm{~cm}$ away from the cavity.

Figure 2 shows the measured transmission spectrum for two configurations, exciting at the center (E2) and detected at D1 and D2. One can notice that, for frequencies less than $3,300 \mathrm{~Hz}$, the amplitude of the signal at the center of the waveguide (D2) is negligible with respect to that at the edge (D1). This means that although both modes $n=0$ and $n=1$ are open mainly the second one is transmitted as can be observed in Fig. 3, where the shapes of the measured modes are shown across the width of the waveguide. Evidence of the opening of the next mode (channel $n=2$ ) is observed around $3,300 \mathrm{~Hz}$ above which a new plateau appears. This is reminiscent of the quantization of the flexural transmission, being the flexural-wave equivalent of the quantization of the electrical conductance ${ }^{18}$. Furthermore, over the smooth part of the transmission, a fluctuating part is observed. These fluctuations are analogous to those appearing in mesoscopic quantum dots ${ }^{2}$ and in microwave cavities ${ }^{6,8}$.

Since the mode with $n=1$ is mainly transmitted, the configurations E1-D1 and E1-D2, where the excitation is close to the edge, are the best suited for the statistical analysis of the transmission fluctuations. In Fig. 4 we show the transmission distributions for two frequency ranges. Assuming an ergodic hypothesis we compare the experimental results with the theoretical predictions from the random matrix theory. For frequencies between $110 \mathrm{~Hz}$ and $210 \mathrm{~Hz}$, it is mainly the mode $n=1$ that is transmitted and the corresponding histogram of the transmission has good agreement with the prediction of random matrix theory for the one channel case, $P(T)=1 / 2 \sqrt{ } T$. For frequencies in the interval $5,540-6,620 \mathrm{~Hz}$, the modes with $n=1$ and $n=2$ are open and the experimental distribution also shows a good agreement with the theoretical predictions ${ }^{5}$. These results are very important since they verify for the first time the simplest model for coherent transport by direct measurement of mode-to-mode transmission.

We have presented measurements of the coherent transmission of mechanical waves through a two-dimensional elastic chaotic cavity with two waveguides. We observed the quantization of the flexural transmission that occurrs when each new mode opens. The statistics of the transmission agree with the theoretical predictions of the simplest model for coherent transport, something that had not been achieved before despite great efforts in the subject. The transmission distribution for two open channels shows better agreement with the 
theory than the one-channel case since there are more resonances within the frequency window. Similar results were observed for different frequency intervals. This demonstrates the universal character of the transmission fluctuations. Although our experimental results are of significance by their own, they open the possibility to verify other transport phenomena without decoherence.

\section{References}

1. Huibers, A. G. et al. Distributions of the Conductance and its Parametric Derivatives in Quantum Dots. Phys. Rev. Lett. 81, 1917-1920 (1998).

2. Keller, M. W. et al. Energy-averaged weak localization in chaotic microcavities. Phys. Rev. B 53, R1693-R1696 (1996).

3. Huibers, A. G., Switkes, M., Marcus, C. M., Campman, K. \& Gossard, A. C. Dephasing in Open Quantum Dots. Phys. Rev. Lett. 81, 200-203 (1998).

4. Büttiker, M. Symmetry of electrical conduction. IBM J. Res. Develop. 32, 317-334 (1988).

5. Baranger, H. U. \& Mello, P. A. Mesoscopic Transport through Chaotic Cavities: A Random S-Matrix Theory Approach. Phys. Rev. Lett. 73, 142-145 (1994).

6. Schanze, H., Alves, E. R. P., Lewenkopf, C. H. \& Stöckmann, H.-J. Transmission fluctuations in chaotic microwave billiards with and without time-reversal symmetry. Phys. Rev. E 64, 065201(R) (2001).

7. Kim, Y.-H., Barth, M., Stöckmann, H.-J. \& Bird, J. P. Wave function scarring in open quantum dots: A microwave-billiard analog study. Phys. Rev. B 65, 165317 (2002).

8. Méndez-Sánchez, R. A., Kuhl, U., Barth, M., Lewenkopf, C. H. \& Stöckmann, H.-J. Distribution of Reflection Coefficients in Absorbing Chaotic Microwave Cavities. Phys. Rev. Lett. 91, 174102 (2003).

9. Schanze, H., Stöckmann, H.-J., Martínez-Mares, M. \& Lewenkopf, C. H. Universal transport properties of open microwave cavities with and without time-reversal symmetry. Phys. Rev. E 71, 016223 (2005).

10. Ławniczak, M., Bauch, S., Hul, O. \& Sirko, L. Experimental investigation of microwave networks simulating quantum chaotic systems: the role of direct processes. Phys. Scr. T147, 014018 (2012).

11. Hemmady, S. et al. Universal properties of two-port scattering, impedance, and admittance matrices of wave-chaotic systems. Phys. Rev. E 74, 036213 (2006).

12. Lobkis, O. I., Rozhkov, I. S. \& Weaver, R. L. Nonexponential Dissipation in a Lossy Elastodynamic Billiard: Comparison with PorterThomas and Random Matrix Predictions. Phys. Rev. Lett. 91, 194101 (2003).

13. Martínez-Argüello, A. M., Martínez-Mares, M., Cobián-Suárez, M., Báez, G. \& Méndez-Sánchez, R. A. A new Fano resonance in measurement processes. EPL 110, 54003 (2015).

14. Søndergaard, N., Guhr, T., Oxborrow, M., Schaadt, K. \& Ellegaard, C. Counting function for a sphere of anisotropic quartz. Phys. Rev. E. 70, 036206 (2005).

15. Gräf, H.-D. et al. Distribution of Eigenmodes in a Superconducting Stadium Billiard with Chaotic Dynamics. Phys. Rev. Lett. 69, 1296-1300 (1992).

16. Morales, A., Gutiérrez, L. \& Flores, J. Improved eddy current driver-detector for elastic vibrations. Am. J. Phys. 69, 517-522 (2001).

17. Manzanarez-Martínez, B. et al. Flexural vibrations of a rectangular plate for the lower normal modes. J. Sound Vib. 329, 5105-5115 (2010).

18. van Wees, B. J. et al. Quantized Conductance of Point Contacts in a Two-Dimensional Electron Gas. Phys. Rev. Lett. 60, 848-850 (1988).

\section{Acknowledgements}

This work was supported by DGAPA-UNAM under project PAPIIT IN103115. E.F.-O. gratefully acknowledges CONACYT for the support grants to pursue his PhD. studies. J.A.F.-V. acknowledge the support of CONACyT under project CB-2010/154586. We acknowledge the kind hospitality of Centro Internacional de Ciencias A. C. and Instituto de Física, Universidad Autónoma de Puebla for group meetings celebrated frequently there. We would like to thank A. Arreola, A. Fernández-Marín, V. Domínguez-Rocha and E. Sadurní for invaluable comments.

\section{Author Contributions}

E.F.-O. made the experiment and the Figures 1-3. A.M.M.-A. analyzed the experimental data and performed the statistical distribution for the flexural transmission and compared with the predictions of random matrix theory shown in Figure 4. M.M.-M. supported the interpretation and statistical analysis of experimental data and wrote the main text of the manuscript. G.B. and R.A.M.-S. designed and directed the experiment and wrote parts of the main text. J.A.F.-V. participated in discussions of results and helped improve the manuscript. All authors reviewed the final version of the manuscript.

\section{Additional Information}

Competing financial interests: The authors declare no competing financial interests.

How to cite this article: Flores-Olmedo, E. et al. Experimental evidence of coherent transport. Sci. Rep. 6, 25157; doi: 10.1038/srep25157 (2016).

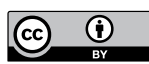

This work is licensed under a Creative Commons Attribution 4.0 International License. The images or other third party material in this article are included in the article's Creative Commons license, unless indicated otherwise in the credit line; if the material is not included under the Creative Commons license, users will need to obtain permission from the license holder to reproduce the material. To view a copy of this license, visit http://creativecommons.org/licenses/by/4.0/ 


\section{Alambres cuánticos}

\section{Fernando Barona, Angel M. Martínez y Moisés Martínez Departamento de Física, Universidad Autónoma Metropolitana, Iztapalapa}

La electricidad y su manipulación han dado origen a grandes avances tanto científicos como tecnológicos desde su descubrimiento en el siglo XVIII. En la actualidad, estos avances han dado lugar a una tendencia de miniaturización de los dispositivos electrónicos, en donde uno de los grandes retos de la ciencia es el transporte de carga con la menor pérdida de energía posible, haciéndolos de esta manera más eficientes; es decir, el sueño de los ingenieros y científicos es construir un alambre con resistencia nula, para lo cual recurren a nuevas forma de comprender el mundo material.

Desde los trabajos de Hans Christian Oersted, Michael Faraday y James Clerk Maxwell sobre el electromagnetismo, y el descubrimiento del electrón en 1897 por Joseph John Thomson, se han desarrollado teorías que nos han ayudado a tener una imagen mental de la estructura de la materia a nivel microscópico. En particular, se han propuesto mecanismos para explicar el fenómeno de la conducción eléctrica en los materiales conocidos como metales. Sin embargo, como lo dicen Neil W. Ashcroft y N. David Mermin en su libro, ${ }^{a}$ mucho podemos entender sobre los no metales conociendo el comportamiento de los metales, éstos últimos se caracterizan por tener un solo electrón en su última capa de valencia, lo que los hace buenos conductores de la electricidad, entre otras propiedades de las cuales carecen los no metales.

Una de las teorías clásicas con más éxito en la explicación de la conducción eléctrica es el modelo propuesto por Paul Drude en 1900, en donde los metales se imaginan como compuestos de al menos dos tipos de partículas: los iones muy pesados e inmóviles, cada uno de los cuales está formado de un núcleo con carga positiva rodeado de una capa interna de electrones que le están fuertemente unidos, y los electrones de conducción cargados negativamente, que vienen de una capa externa de electrones débilmente unidos al núcleo y que vagan libremente a través del material. Estos últimos se consideran como un gas diluido de esferas sólidas cargadas, con movimiento aleatorio que se puede describir por medio de la teoría cinética de los gases. En ausencia de un campo eléctrico externo, el flujo neto de carga en cualquier dirección es nulo. Pero como el gas está cargado, éste responde cuando se aplica un campo eléctrico a través de un voltaje.

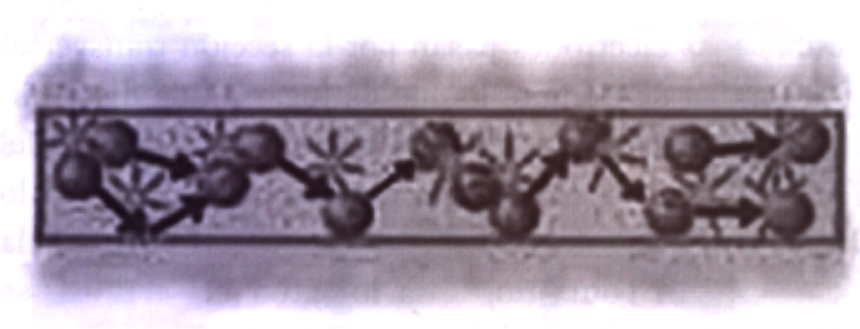

Figura 1. Esquema de las colisiones inelásticas que sufren los electrones en su viaje a través de un conductor [www.asifunciona.com).

\section{La ley de Ohm}

Cuando se aplica un voltaje a un conductor, los electrones se mueven en línea recta de acuerdo con las leyes de Newton. Sin embargo, pueden chocar o dispersarse inelásticamente con los iones, defectos o impurezas que hay en el interior del material y que cambian abruptamente su

a Solid State Physics, N. W. Ashcroft y N. D. Mermin, Cengage Learning (1976). 
velocidad, en módulo y dirección, perdiendo energía cinética en cada choque. Aún así los electrones son arrastrados con una velocidad media durante un tiempo promedio entre choques; es decir, un electrón puede viajar libremente una distancia promedio conocida como camino libre medio inelástico (ver Fig. 1). Las múltiples colisiones inelásticas dan lugar a una oposición al flujo de electrones, o resistencia, que se puede interpretar como un fenómeno de fricción, y la energía que se disipa se convierte en calor por el efecto Joule. Es por esta razón que los conductores se calientan al transportar una corriente eléctrica.

Georg Simon Ohm definió y cuantificó la resistencia eléctrica como la cantidad que relaciona el flujo neto de carga que atraviesa una sección transversal, en la unidad de tiempo, llamada intensidad de corriente, con el voltaje aplicado. Lo que sabemos de la resistencia eléctrica es que debe depender de alguna cantidad que caracteriza a cada material, pues no es lo mismo un conductor de oro que uno de plata o cobre. Lo que distingue a los distintos conductores es su resistividad, que es una cantidad intrínseca de cada material a través de la configuración de dispersores en su interior. También sabemos que la resistencia depende de factores geométricos. Por ejemplo, para un alambre recto la resistencia aumenta linealmente con la longitud y disminuye con el área de la sección transversal, lo cual tiene sentido porque mientras más ancho es el alambre más electrones pasan por la sección transversal en la unidad de tiempo, lo que da lugar a una disminución de la resistencia. Por otra parte, si la longitud del alambre aumenta, más y más dispersores hay en él a lo largo del camino de los electrones, aumentando así la resistencia. Al contrario, si la longitud del alambre se hace cada vez más pequeña, uno debería esperar que la resistencia fuera cada vez menor hasta llegar a ser cero: es decir, no habría resistencia en un alambre suficientemente pequeño. Pero la ley de $\mathrm{Ohm}$ es válida para conductores macroscópicos, de tamaño mucho más grande que la distancia media entre dispersor y dispersor, de tal manera que no podríamos hacer la longitud tan pequeña como queramos. A escalas pequeñas la longitud la ley de $\mathrm{Ohm}$ deja de ser válida porque no tendría sentido hablar de una resistividad propia de un material específico. Entramos así en un nuevo régimen donde aparecen fenómenos nuevos.

\section{La resistencia cuántica}

Cuando el tamaño del sistema se vuelve menor que la distancia promedio entre dispersores, los conductores no tienen impurezas en su interior que pudieran dispersar inelásticamente a los electrones. De alguna manera carece de sentido hablar de un material específico caracterizado por una resistividad, no hay distinción entre materiales conductores. Resulta que en las épocas actuales la ciencia y la tecnología aceptaron el reto de construir dispositivos diminutos de tamaño menor que la distancia media entre dispersores, y a temperaturas del orden del milikelvin para evitar los efectos inelásticos. De esta manera, los electrones pueden viajar libremente sin colisionar con nada, excepto con las fronteras del dispositivo conductor mismo, !no hay colisiones inelásticas y tampoco disipación de energía! Es decir, dichos dispositivos no están hechos de material alguno y se conocen como sistemas mesoscópicos cuánticos: son "pequeños conductores cuyas dimensiones están entre lo microscópico y lo macroscópico... Son mucho más grandes que los objetos microscópicos como los átomos, pero no lo suficientemente grandes como para ser ohmicos". ${ }^{\prime \prime}$ En ellos el transporte de electrones se establece de manera libre y coherente, y su comportamiento está gobernado por las leyes de la mecánica cuántica. Es decir, los electrones ya no se pueden describir como un gas de esferas sólidas sino que se comportan como ondas, las cuales pueden sufrir efectos de interferencia al ser dispersadas por los defectos o impurezas, de la misma forma en que sucede con otro tipo de ondas como las del agua. Ejemplos de sistemas mesoscópicos son los dispositivos micro y nanoelectrónicos conocidos como dispositivos cuánticos balísticos. Estos dispositivos se pueden construir sobre un gas bidimensional de electrones libres, formado en la interfaz entre dos heteroestructuras semiconductoras, las

b Tomado de Electronic Transport in Mesoscopic Systems, Supriyo Datta, Cambridge University Press (1995). 
más típicas son Arsenuro de Galio (GaAs) y Arsenuro de Galio Aluminio (AlGaAs). Sobre esta interfaz se colocan contactos metálicos puestos a potencial fijo que al repeler los electrones que están justo debajo (algunos nanómetros) da la forma del conductor.

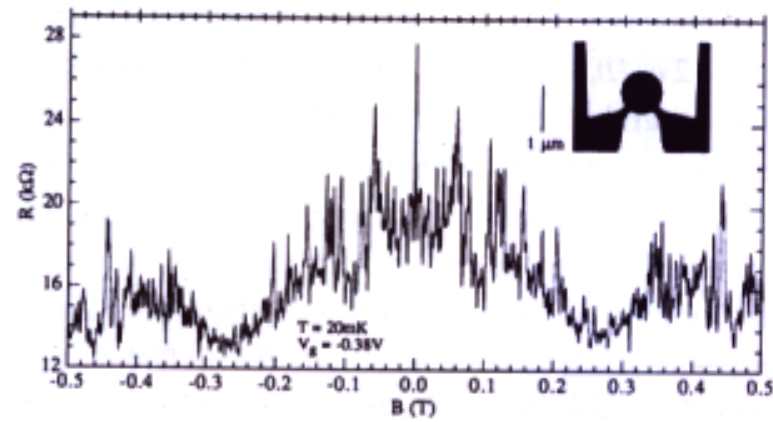

Figura 2. Resistencia $R(k \Omega)$ de un conductor balístico plano circular en función del campo magnético externo aplicado $B(T)$. [Figure 1 adapted with permission from Physical Review Letters 69, 506 (1992). Copyrighted by the American Physical Society.]

En la Fig. 2 se encuentra una micrografía de un conductor balístico plano con forma circular de una micra de diámetro. A este tipo de sistemas también se les conoce como puntos cuánticos, o de cero dimensiones, por confinar el movimiento de los electrones en las direcciones $x, y$ y $z$. Los electrones entran al punto cuántico a través de contactos también balísticos (región angosta) desde los reservorios electrónicos (zona oscura fuera del conductor). Una vez dentro del sistema, los electrones sólo pueden chocar con las paredes que los confinan. Nuestra intuición basada en la ley de Ohm nos dice que la resistencia debería ser nula. Sin embargo, en la misma figura se muestra el resultado de la medición de la resistencia para diferentes valores de un campo magnético externo, aplicado perpendicularmente al plano del conductor. Puede verse claramente que la resistencia nunca es cero, de hecho muestra fluctuaciones con respecto al campo magnético. Pero, ide dónde viene la resistencia si no hay disipación de energía? Si no hay dispersores en el interior del conductor, la resistencia debe venir de la conexión con el mundo macroscópico, por lo que el proceso de medición resulta importante.

\section{Resistencia de dos puntos}

Las mediciones típicas de la resistencia utilizan puntas de medición que se colocan en los dos extremos del conductor, en los reservorios que establecen el flujo de carga (ver la Fig. 3a). Estos reservorios se comportan como los conductores ohmicos macroscópicos; es ahí donde ocurren los procesos inelásticos que son los responsables de la disipación de la energía y por ende, de una resistencia. A esta resistencia se le conoce como resistencia de dos puntos (o de dos terminales). Esto explica el resultado del experimento mostrado en la Fig. 2. Las fluctuaciones que se observan se deben a un fenómeno de interferencia cuántica de las ondas electrónicas inducida por la geometría del sistema. Nuevamente, aún en esta escala microscópica la resistencia depende de la geometría del conductor. Pero se espera que estas fluctuaciones no ocurran en un alambre recto.

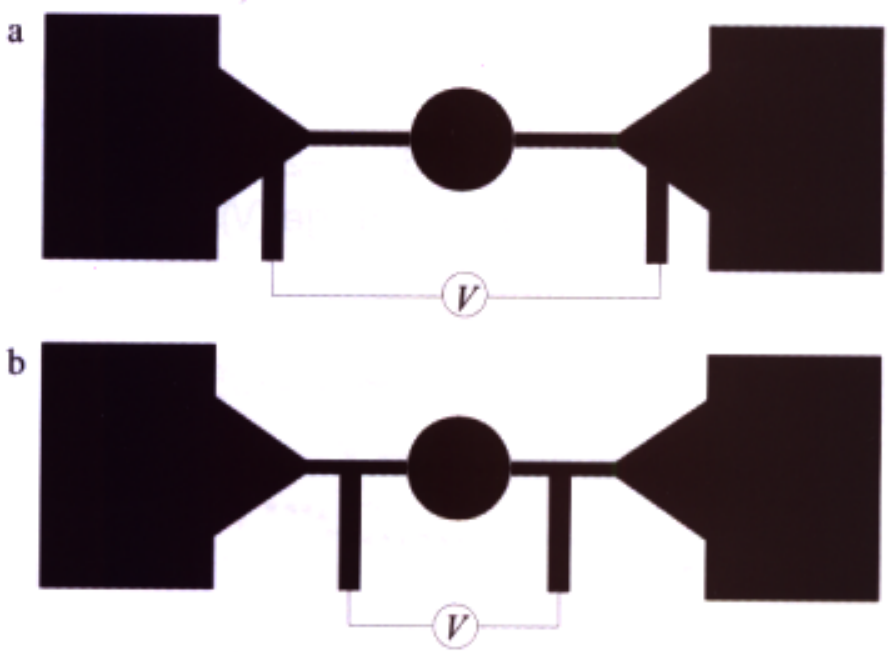

Figura 3. Medición de la resistencia en la configuración de dos $|a|$ y cuatro (b) puntos.

En la Fig. 4a se muestra un esquema usado para medir la resistencia de un alambre recto (One-dimensional wire) formado entre dos reservorios electrónicos (Source 2DEG y Drain 2DEG). Por medio de una compuerta de voltaje (Gate voltage, no mostrado en la figura) se puede controlar el ancho del alambre permitiendo un cierto 


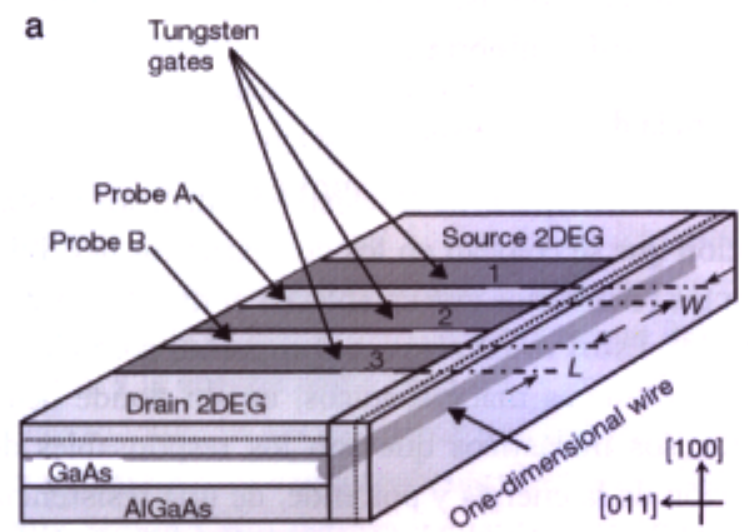

b

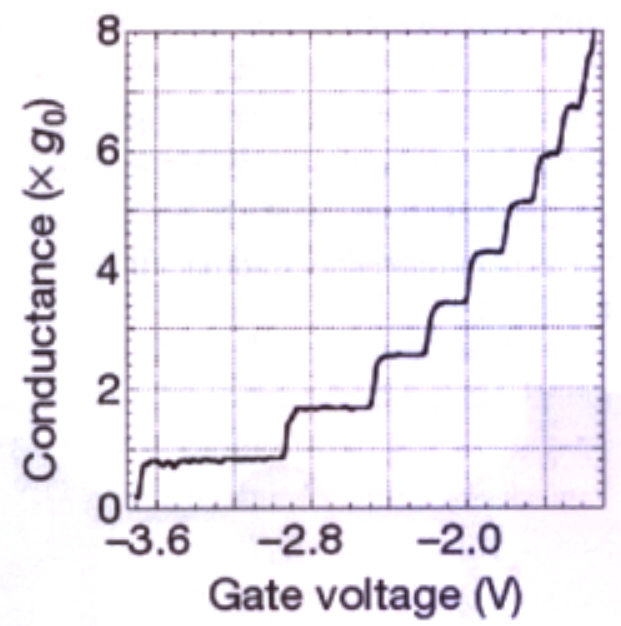

c

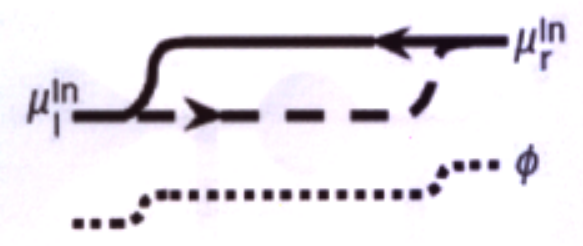

Figura 4. Dispositivo electrónico usado para medir la resistencia. (a) Activando sólo la compuerta 2 se tiene la configuración de dos puntos; si se activan las compuertas 1,2 y 3 se obtiene la resistencia de cuatro puntos. (b) La conductancia (recíproco de la resistencia) en la configuración de dos puntos muestra un comportamiento de escalera conforme se abren los modos propagantes. [Reprinted by permission from Macmillan Publishers Ltd: Nature (Vol 411, Issue 6833, Pages 51.54] 0 Macmillan Magazines Ltd [2001), www.nature.com.]

número de modos o formas de propagación de los electrones debido al confinamiento transversal. Recordemos que los electrones se comportan como ondas y que su longitud de onda a lo más puede valer dos veces el ancho del conductor. En general sólo ciertas longitudes de onda son permitidas, lo que da lugar a los distintos modos de propagación. Las compuertas 1, 2 y 3 (Tungsten gates) se usan para formar las dos puntas de medición (Probe A y Probe B). La configuración de dos puntos se obtiene activando sólo la compuerta 2. En este caso la medición del recíproco de la resistencia, la conductancia, medida en unidades de $2 e^{2} / h$, donde $h$ es la constante de Planck, se muestra en la Fig. $4 \mathrm{~b}$ en función del voltaje de compuerta que controla el ancho del alambre. Se observa que la conductancia no fluctúa pero muestra un comportamiento de escalera que se debe a la equipartición de la corriente en los distintos modos de propagación de los electrones en el alambre. Cuando no hay ningún modo abierto, la conductancia es cero pues no puede haber propagación alguna, el alambre es tan angosto comparado con longitud de onda que no cabe la posibilidad de propagar electrones. Conforme aumenta el voltaje de compuerta el alambre se hace más ancho, abriendo la posibilidad de más modos posibles de propagación; cada vez que se abre un nuevo modo la conductancia aumenta una unidad en $2 e^{2} / h$. Esto quiere decir que la conductancia está cuantizada y a la cantidad $2 e^{2} / h$ se le conoce como cuanto de conductancia. Una cuestión interesante se tiene en el caso de un modo propagante, ya que en ese caso la resistencia toma el valor fijo no nulo de $h / 2 e^{2} \approx 13 \mathrm{k} \Omega$. El valor de esta resistencia es el resultado de la suma en serie de las dos resistencias de contacto con los reservorios electrónicos. Sin embargo, persiste el debate de si la resistencia de dos puntos corresponde o no a la del conductor, o se debe sólo al efecto de la interacción de éste con los reservorios. Una configuración que se cree es la más adecuada para medir la resistencia intrínseca de un conductor es la de cuatro puntos, como se muestra en la Fig. $3 \mathrm{~b}$.

\section{Resistencia de cuatro puntos}

Activando las tres compuertas en el esquema del dispositivo mostrado en la Fig. 4a, las puntas de medición quedan sobre el alambre, separadas de los reservorios electrónicos. Esta configuración 
permite medir la resistencia intrínseca del alambre balístico, conocida como resistencia de cuatro puntos (o de cuatro terminales).

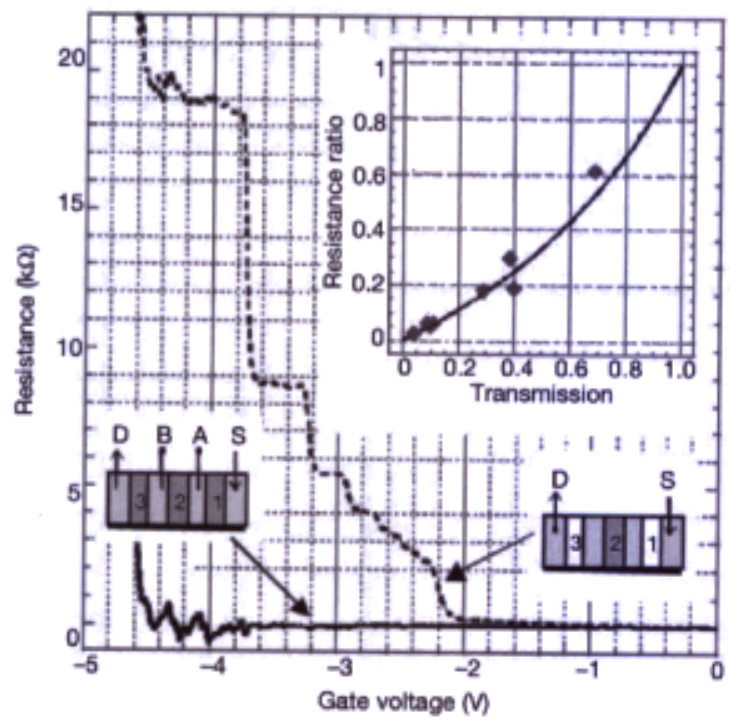

Figura 5: Resistencia de cuatro puntos. Reprinted by permission from Macmillan Publishers Ltd: Nature (Vol 411, Issue 6833, Pages 51-54) o Macmillan Magazines Ltd [2001], www.nature.com.

El resultado de la medición de la resistencia de cuatro puntos, se muestra en la Fig. 5 en línea con- tinua. Como puede observarse, la resistencia es nula aún cuando el voltaje de compuerta disminuye, permitiendo un número menor de modos de propagación. Este resultado concuerda con lo que esperaríamos para un conductor limpio y sin disipación. Para pocos modos de propagación se tienen algunas fluctuaciones pero pueden deberse al acoplamiento de las puntas de medición con el alambre, formando así un punto cuántico equivalente al de la Fig 3. Por comparación también se muestra la resistencia en la configuración de dos puntos en línea discontinua.

En resumen, todo parece indicar que la resistencia de un alambre cuántico balístico está de acuerdo con nuestra intuición. Es un hecho que ya no podemos hablar de la naturaleza de un conductor en sí, pero la resistencia sigue dependiendo de la geometría de los conductores. Además, la resistencia es nula en ausencia de defectos o impurezas que obstaculicen el libre flujo de los electrones, haciéndoles perder energía. Aunque el sueño de todo ingeniero podría ser la construcción de dispositivos pequeños con la menor pérdida de energía posible, las propiedades de transporte electrónico se verían afectadas por los reservorios electrónicos, donde la disipación ocurre inevitablemente, si los deseamos conectar con el mundo macroscópico en que vivimos. 


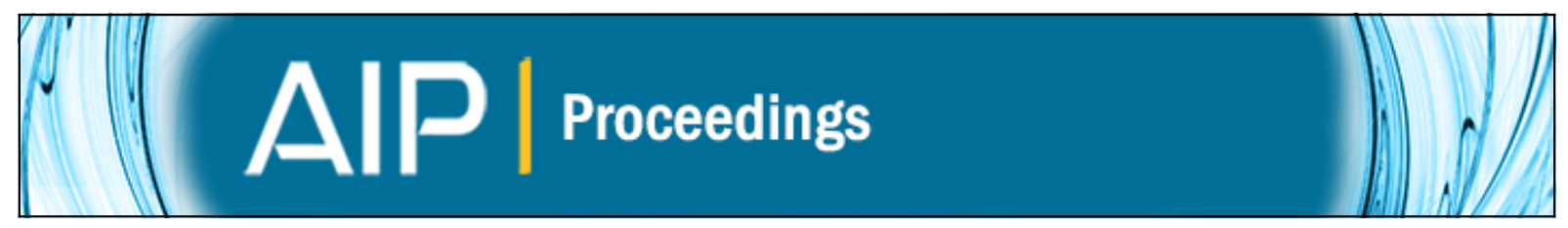

\section{Random matrix study for a three-terminal chaotic device}

A. M. Martínez-Argüello, E. Castaño, and M. Martínez-Mares

Citation: AIP Conference Proceedings 1579, 46 (2014); doi: 10.1063/1.4862416

View online: http://dx.doi.org/10.1063/1.4862416

View Table of Contents: http://scitation.aip.org/content/aip/proceeding/aipcp/1579?ver=pdfcov

Published by the AIP Publishing 


\title{
Random matrix study for a three-terminal chaotic device
}

\author{
A. M. Martínez-Argüello, E. Castaño and M. Martínez-Mares \\ Departamento de Física, Universidad Autónoma Metropolitana-Iztapalapa, Apartado Postal 55-534, 09340 \\ México Distrito Federal, Mexico
}

\begin{abstract}
We perform a study based on a random-matrix theory simulation for a three-terminal device, consisting of chaotic cavities on each terminal. We analyze the voltage drop along one wire with two chaotic mesoscopic cavities, connected by a perfect conductor, or waveguide, with one open mode. This is done by means of a probe, which also consists of a chaotic cavity that measure the voltage in different configurations. Our results show significant differences with respect to the disordered case, previously considered in the literature.
\end{abstract}

Keywords: quantum transport, electronic devices, multiple scattering PACS: 73.23.-b, 73.21.Hb, 72.10.-d

\section{INTRODUCTION}

In the last thirty years there have been much theoretical and experimental work concerning electronic transport through multiterminal devices (see Refs. [1,2] there in). Nowadays, the interest to study the electronic transport properties on these devices has been renewed [3-8], due to the fact that they are very useful in experimental measurements in several configurations [9-11].

The earlier experiments were done with normal metal conductors, whose random distribution of impurities in their microscopic structure, give rise to interference that is reflected in the relevant physical observables, like resistance or voltage measurements. Moreover, those quantities show sample to sample fluctuations [12-14]. More recently, the interest on these systems has resurged due to recent advances in technology, that allow to access to clean devices, where the typical size is smaller than the elastic mean free path. Therefore, the electrons propagate ballistically and scattering is produced only by the device boundaries, which have important consequences in the electronic transport through the device $[6,15,16]$. For instance, when the geometry is such that the classical dynamics in the system is chaotic, the transport properties fluctuates too [17-19]. What is very important is to know how are the fluctuations with respect to the disordered case.

In this work, by numerical simulation, we analyze the statistical distribution of the voltage drop along a chaotic wire, which consists of two chaotic mesoscopic cavities connected by a perfect conductor with one open mode. The probe is a chaotic cavity that measure the voltage in different configurations. The presence and absence of time reversal invariance are considered. We compare our results with the ones obtained in an equivalent three terminal device but with disordered, instead of chaotic, wires, previously studied in the literature, where the distribution of the voltage drop was determined in the presence of time reversal invariance only $[12,13]$. There, a remarkable difference in the distribution of the voltage drop between the ballistic regime and the strong disordered limit, has been found. The position of the probe has a stronger effect than in the disordered case.

First, we summarize the scattering formalism for the voltage drop in a three terminal device, proposed by Büttiker $[10,11]$. Then, we construct the scattering matrix for our system, in terms of the scattering matrices of the individual cavities, as well as of the scattering matrix associated to the junction, for which we assume the simplest model introduced by Büttiker, that couple the probe symmetrically to the horizontal wire [9]. For the statistical analysis, we make an ensemble of systems by assuming that the scattering matrix of each cavity is chosen from a Circular Ensemble, Orthogonal or Unitary, depending on the presence or absence of time reversal invariance. We present our conclusions at the end.

(C) 2014 AIP Publishing LLC 978-0-7354-1209-5/ $\$ 30.00$ 


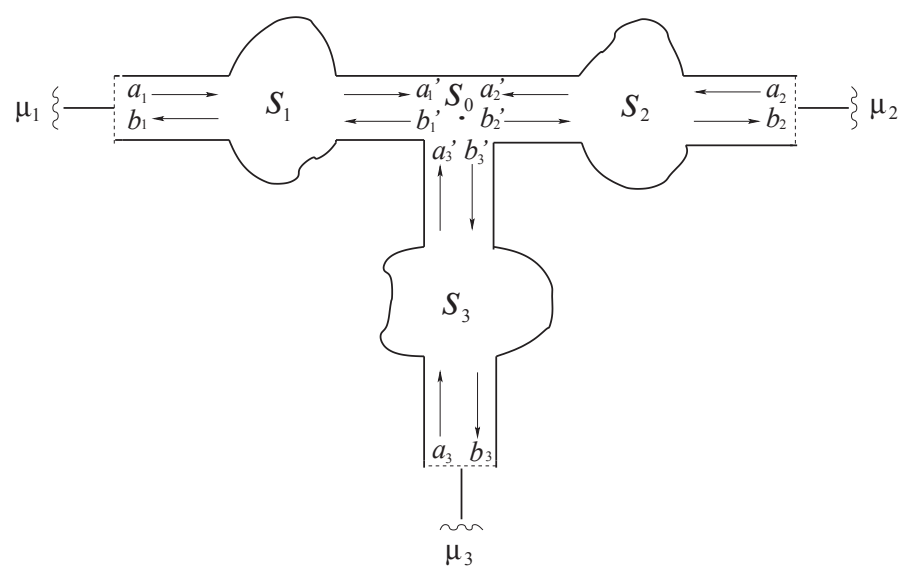

FIGURE 1. A scattering system consisting of three one-dimensional wires converging to a junction. The thin lines represent perfect conductors that connect the wave guides to the chemical reservoirs. The amplitude of the incoming (outgoing) wave in wire $i$ is denoted by $a_{i}\left(b_{i}\right)$, while $a_{i}^{\prime}\left(b_{i}^{\prime}\right)$ denotes the amplitude at the junction. Each wire is described by a $2 \times 2$ scattering matrix $S_{j}$ and the junction by a $3 \times 3$ matrix, $S_{0}$.

\section{ELECTRONIC TRANSPORT IN A THREE-TERMINAL SYSTEM}

In the formulation of Landauer-Büttiker, the electronic transport is reduced to a scattering problem. In a single mode multiprobe devices, the current $I_{i}$ in a lead $i$ can be written into two components, one being the reflection to the same lead and the transmission from the others leads to the lead $i$. That is, $I_{i}$ is given in terms of the reflection and transmission coefficients, according to [11]

$$
I_{i}=\frac{e}{h}\left[\left(1-R_{i i}\right) \mu_{i}-\sum_{j \neq i} T_{i j} \mu_{j}\right],
$$

where $\mu_{j}$ is the chemical potential in lead $j, R_{i i}$ is the reflection coefficient to the lead $i$, and $T_{i j}$ represent the transmission from lead $j$ to lead $i$. These coefficients are given by the scattering matrix $S$ as $R_{i i}=\left|S_{i i}\right|^{2}$ and $T_{i j}=\left|S_{i j}\right|^{2}$.

The voltage along a horizontal wire, connected via perfect leads to two reservoirs of fixed chemical potentials, $\mu_{1}$ and $\mu_{2}$, can be measured in a three terminal device, where the third wire is in a voltage measurement configuration (see Fig. 1); that is, the chemical potential $\mu_{3}$ is such that the current through it is equal to zero, $I_{3}=0$. In such a case [11],

$$
\mu_{3}=\frac{1}{2}\left(\mu_{1}+\mu_{2}\right)+\frac{1}{2}\left(\mu_{1}-\mu_{2}\right) f
$$

where $f$ is given by

$$
f=\frac{T_{31}-T_{32}}{T_{31}+T_{32}} .
$$

Equation (2) shows that the chemical potential $\mu_{3}$ has an averaged part, that comes from the effect of the resorvoirs $\mu_{1}$ and $\mu_{2}$ only. The second part gives the deviation from the averaged part, and depends on the intrinsic nature of the conductors through the quantity $f$, that contains all the relevant information about the multiple scattering in the device. If the conductors are disordered or chaotic, $f$ fluctuates between -1 and 1 , since $\mu_{3}$ can not reach the values $\mu_{1}$ nor $\mu_{2}$ due to the contact resistance [11]. The disordered three terminal device was studied in Refs. [12, 13].

In what follows we will consider a three terminal device where the conductors are chaotic. Since $f$ depends on the scattering matrix $S$ of the whole system, we construct $S$ in terms of the scattering matrices of each conductor and the scattering matrix of the splitter, that we will assume to be known. For the splitter we assume a simple model, while the scattering matrices of the chaotic conductors are chosen from an ensemble of random matrices that satisfy certain symmetry requirements. 


\section{THE $S$ MATRIX FOR A THREE TERMINAL DEVICE}

Let us consider the three terminal system shown in Fig. 1. The system is described by the scattering matrix $S$ which relates the incoming plane waves amplitudes on each terminal, $a_{1}, a_{2}$, and $a_{3}$, to the outgoing ones, $b_{1}, b_{2}$, and $b_{3}$, by

$$
\left(\begin{array}{l}
b_{1} \\
b_{2} \\
b_{3}
\end{array}\right)=S\left(\begin{array}{l}
a_{1} \\
a_{2} \\
a_{3}
\end{array}\right)
$$

where we assume that $S$ contains all the information that from the system we can obtain. Of course, $S$ depends on the scattering process inside the system, due to scattering elements.

Let assume that the splitter is represented by the scattering matrix $S_{0}$, that couples the three terminals; therefore, the amplitudes at the junction are related as

$$
\left(\begin{array}{l}
b_{1}^{\prime} \\
b_{2}^{\prime} \\
b_{3}^{\prime}
\end{array}\right)=S_{0}\left(\begin{array}{l}
a_{1}^{\prime} \\
a_{2}^{\prime} \\
a_{3}^{\prime}
\end{array}\right)
$$

If the conductors on each terminal are represented by the scattering matrices $S_{j}(j=1,2,3)$, the amplitudes are related as follows:

$$
\left(\begin{array}{l}
b_{1} \\
a_{1}^{\prime}
\end{array}\right)=S_{1}\left(\begin{array}{l}
a_{1} \\
b_{1}^{\prime}
\end{array}\right), \quad\left(\begin{array}{l}
a_{2}^{\prime} \\
b_{2}
\end{array}\right)=S_{2}\left(\begin{array}{l}
b_{2}^{\prime} \\
a_{2}
\end{array}\right), \quad\left(\begin{array}{l}
a_{3}^{\prime} \\
b_{3}
\end{array}\right)=S_{3}\left(\begin{array}{c}
b_{3}^{\prime} \\
a_{3}
\end{array}\right),
$$

where each matrix $S_{j}$ is a $2 \times 2$ matrix with the general structure

$$
S_{j}=\left(\begin{array}{cc}
r_{j} & t_{j}^{\prime} \\
t_{j} & r_{j}^{\prime}
\end{array}\right)
$$

with $r_{j}, t_{j}$ are the reflection and transmission amplitudes when the incidence is from the left (or below for $j=3$ ) of the $j$ th conductor, and $r_{j}^{\prime}, t_{j}^{\prime}$ when the incidence is from the other side. Flux conservation implies that $S_{j}$ is unitary,

$$
S_{j} S_{j}^{\dagger}=I_{2}
$$

where $I_{2}$ stands for the $2 \times 2$ identity matrix. Equation (8) is the only requirement in absence of any symmetry, while in the presence of time reversal invariance, $S_{j}$ is a unitary and symmetric,

$$
S_{j}=S_{j}^{T}
$$

where $T$ stands for the transposed.

Through Eqs. (5), (7) and (6) we arrive to the scattering matrix $S$ that describes the full system, which is given by

$$
S=S_{P P}+S_{P Q} S_{0} \frac{1}{1-S_{Q Q} S_{0}} S_{Q P}
$$

where we have defined

$$
S_{P P}=\left(\begin{array}{ccc}
r_{1} & 0 & 0 \\
0 & r_{2}^{\prime} & 0 \\
0 & 0 & r_{3}^{\prime}
\end{array}\right), \quad S_{P Q}=\left(\begin{array}{ccc}
t_{1}^{\prime} & 0 & 0 \\
0 & t_{2} & 0 \\
0 & 0 & t_{3}
\end{array}\right), \quad S_{Q P}=\left(\begin{array}{ccc}
t_{1} & 0 & 0 \\
0 & t_{2}^{\prime} & 0 \\
0 & 0 & t_{3}^{\prime}
\end{array}\right), \quad S_{Q Q}=\left(\begin{array}{ccc}
r_{1}^{\prime} & 0 & 0 \\
0 & r_{2} & 0 \\
0 & 0 & r_{3}
\end{array}\right) .
$$

Equation (10) has a nice interpretation: the first term on the right hand side, $S_{P P}$, represents the reflected parts of the waves that reach the conductors, while the second term comes from the multiple scattering in the system. Here, $S_{Q P}$ gives the transmission from outside to inside, $S_{P Q}$ gives the transmission from inside to outside of the system, and $S_{Q Q}$ represents the internal reflections.

Notice that $S$ is also a unitary matrix once we ensure that $S_{0}$ is chosen as a unitary matrix too, and the symmetry conditions are fixed by the symmetry properties of the $S_{j}$ 's. Although our result is general, in what follows we adopt a simple model for $S_{0}$ and we choose $S_{j}$ from an ensemble of scattering matrices that simulates chaotic cavities. 


\section{A simple model for the splitter}

A simple model for the $S$-matrix of the splitter, real and symmtric, that couples the probe symmetrically, was proposed by Büttiker [9], namely

$$
S_{0}=\left(\begin{array}{ccc}
a & b & \sqrt{\varepsilon} \\
b & a & \sqrt{\varepsilon} \\
\sqrt{\varepsilon} & \sqrt{\varepsilon} & -(a+b)
\end{array}\right),
$$

where $\varepsilon$ is a real parameters with $0 \leq \varepsilon \leq 1 / 2$, which gives the coupling strength, and

$$
a=-\frac{1}{2}(1-\sqrt{1-2 \varepsilon}), \quad b=+\frac{1}{2}(1+\sqrt{1-2 \varepsilon}) .
$$

When the coupling vanishes ( $\varepsilon \rightarrow 0$ ), $a \rightarrow 0$ and $b \rightarrow 1$ which means that the probe is decoupled and there is complete transmission between the terminals 1 and 2 . On the contrary, when the probe is perfectly coupled $(\varepsilon=1 / 2), a=-1 / 2$ and $b=1 / 2$, nothing is reflected to the probe.

\section{THE VOLTAGE MEASUREMENT}

We assume that the conductors in our device are in fact chaotic cavities, such that the voltage measurement, and any other transport properties, shows sample to sample fluctuations, although macroscopically seems to be identical; this is due to the difficulty of control of the shape of the cavity microscopically. Of course, the fluctuations also arise with respect to external parameters like the chemical potentials and magnetic field. Therefore, we require to make a statistical study for the voltage measurement. We do this for two kind of ensembles for the $S_{j}$ matrices: in presence and absence of time reversal symmetry. In the Dyson's scheme these correspond to the orthogonal and unitary cases, labeled by $\beta=1$ and $\beta=2$, respectively [20].

\section{Presence of time reversal invariance}

In the $\beta=1$ symmetry, an $S_{j}$ matrix can be parameterized in a "polar representation" as [17]

$$
S_{j}=\left(\begin{array}{cc}
\mathrm{e}^{\mathrm{i} \phi_{j}} & 0 \\
0 & \mathrm{e}^{\mathrm{i} \psi_{j}}
\end{array}\right)\left(\begin{array}{cc}
-\sqrt{1-\tau_{j}} & \sqrt{\tau_{j}} \\
\sqrt{\tau_{j}} & \sqrt{1-\tau_{j}}
\end{array}\right)\left(\begin{array}{cc}
\mathrm{e}^{\mathrm{i} \phi_{j}} & 0 \\
0 & \mathrm{e}^{\mathrm{i} \psi_{j}}
\end{array}\right),
$$

where $\phi_{j}$ and $\psi_{j}$ are random numbers, uniformly distributed in the interval $[0,2 \pi]$, and $\tau_{j}$ is randomly distributed in $[0,1]$. The probability distribution for $S_{j}$ is [17]

$$
d P_{1}\left(S_{j}\right)=\frac{d \tau_{j}}{2 \sqrt{\tau_{j}}} \frac{d \phi_{j}}{2 \pi} \frac{d \psi_{j}}{2 \pi}
$$

which defines the Circular Orthogonal Ensemble, which can be generated numerically. Once this is done, we substitute the elements of the $S_{j}$ matrices in the expressions given in Eq. (11), and then in Eq. (10) from which we obtain the transmission coefficients $T_{31}$ and $T_{32}$, needed to determine $f$ through Eq. (3).

The numerical results for the distribution of $f$, for several values of the coupling strength $\varepsilon$, are shown in Fig. 2 for different measurement configurations. Panels (a), (b), and (c) of this figure are the most general cases of voltage measurements with respect to the position, where all conductors are chaotic. We can observe a clear dependence on the position of the probe. In panel (a), the probe is in the middle of the horizontal wire, and the distribution of $f$ is symmetric around zero, which means that $\mu_{3}$ fluctuates symmetrically around the average $\left(\mu_{1}+\mu_{2}\right) / 2$. However, when the position of the probe changes to one end of the horizontal wire, the distribution of $f$ is no more symmetric with respect to zero, as can be seen in panels (b) and (c) of Fig. 2; in fact, $\mu_{3}$ tends to be closer to the chemical potential of that terminal. We also note that the distribution of $f$ is independent of the coupling parameter. However, when the probe is asymmetrically located in the horizontal wire, the distribution of $f$ reminds that of the probe in the midpoint. We can see that our results contrast with the disordered case of Refs. $[12,13]$ in both limits of weak and strong disorder. 

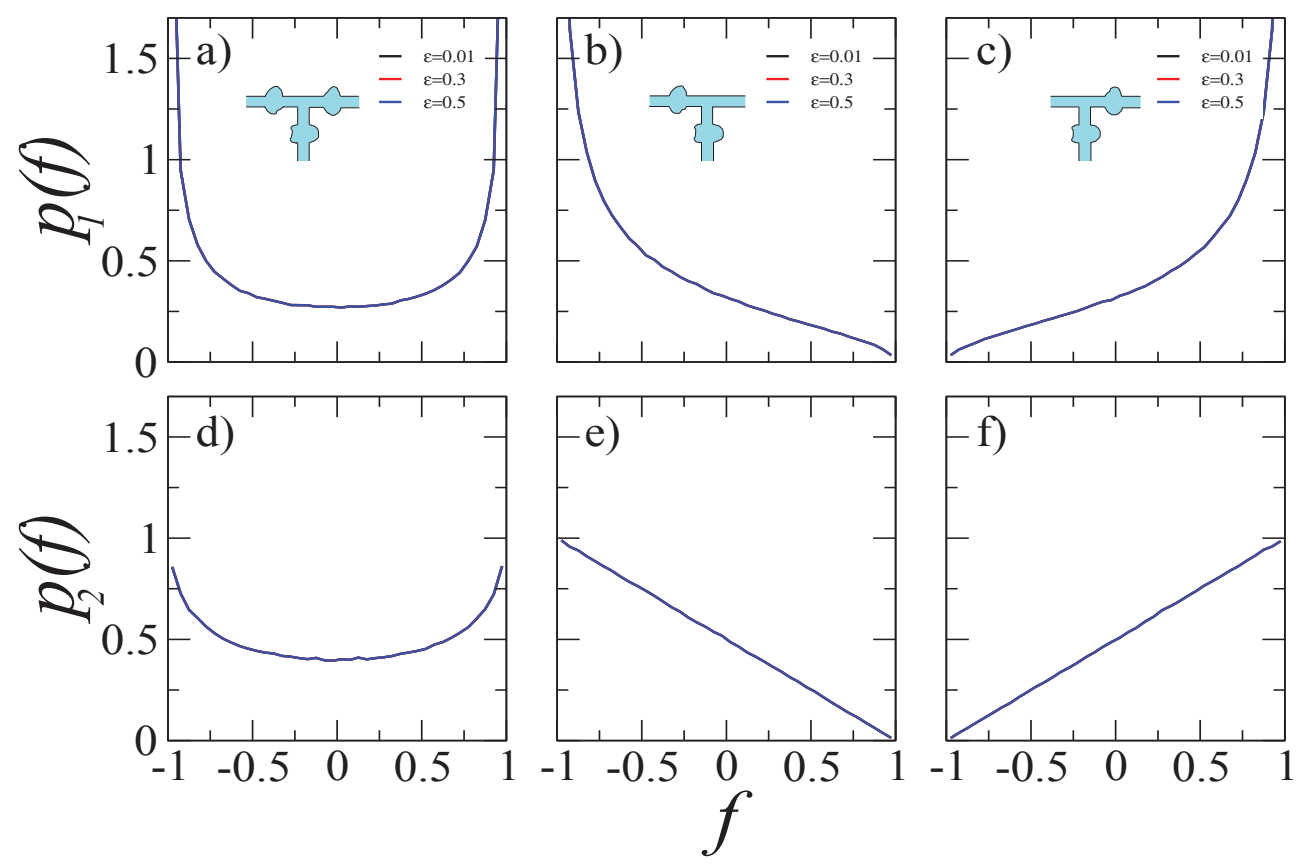

FIGURE 2. Distribution of $f$ for a chaotic three terminal device for different values of $\varepsilon$ and configurations (insets), (a), (b) and (c) in the presence, and (d), (e) and (f) in the absence, of time reversal invariance.

\section{Absence of time reversal invariance}

The scattering matrix $S_{j}$ for the $\beta=2$ symmetry has the following parametrization [17],

$$
S_{j}=\left(\begin{array}{cc}
\mathrm{e}^{\mathrm{i} \phi_{j}} & 0 \\
0 & \mathrm{e}^{\mathrm{i} \psi_{j}}
\end{array}\right)\left(\begin{array}{cc}
-\sqrt{1-\tau_{j}} & \sqrt{\tau_{j}} \\
\sqrt{\tau_{j}} & \sqrt{1-\tau_{j}}
\end{array}\right)\left(\begin{array}{cc}
\mathrm{e}^{\mathrm{i} \phi_{j}^{\prime}} & 0 \\
0 & \mathrm{e}^{\mathrm{i} \psi_{j}^{\prime}}
\end{array}\right) .
$$

Here, the probability distribution of $S_{j}$ is given by [17]

$$
d P_{2}\left(S_{j}\right)=d \tau_{j} \frac{d \phi_{j}}{2 \pi} \frac{d \psi_{j}}{2 \pi} \frac{d \phi_{j}^{\prime}}{2 \pi} \frac{d \psi_{j}^{\prime}}{2 \pi}
$$

which defines the Circular Unitary Ensemble.

In Fig. 2, panels (d), (e) and (f), we show the results for the distribution of $f$ for the same values of $\varepsilon$ and configurations as in the $\beta=1$ case. The dependence on the intensity of the coupling, as well as in the position of the probe, is also observed. As in the $\beta=1$ case, the distribution of $f$ is independent of $\varepsilon$ and has memory with respect to the measurement in the midpoint of the horizontal wire. What is important to note here is that the distribution of $f$ is strongly affected by the broken symmetry of time reversal.

\section{CONCLUSIONS}

We studied the voltage drop along a horizontal wire with one open mode, consisting of chaotic conductors, in a three terminal device. This was done by using a probe which is chaotic. Our analysis was based on random matrix theory simulations for the chaotic elements, in the presence and absence of time reversal invariance. We found a clear dependence of the position of the probe in the horizontal wire. Also, we found a strong dependence of the time reversal symmetry. 


\section{ACKNOWLEDGMENTS}

The authors thank the organizers of the V Leopoldo García-Colín Mexican Meeting for their kind invitation. AMM-A acknowledges financial support from CONACyT, Mexico. MM-M is a fellow of Sistema Nacional de Investigadores, Mexico; he also thanks MA Torres-Segura for her encouragement.

\section{REFERENCES}

1. S. Datta, Electronic Transport in Mesoscopic Systems, Cambridge University Press, Cambridge, 1995.

2. S. Goodnick, IEEE Transactions on Nanotechnology 2, 368-385 (2003).

3. R. de Picciotto, H. L. Stormer, L. N. Pfeiffer, K. W. Baldwin, and K. W. West, Nature 411, 51-54 (2001).

4. A. M. Chang, Nature 411, 39-40 (2001).

5. R. Fleischmann and T. Geisel, Phys. Rev. Lett. 89, 016804 (2002).

6. S. de Haan, A. Lorke, J. P. Kotthaus, W. Wegscheider, and M. Bichler, Phys. Rev. Lett. 92, 056806 (2004).

7. B. Gao, Y. F. Chen, M. S. Fuhrer, D. C. Glattli, and A. Bachtold Phys. Rev. Lett. 95, 196802 (2005).

8. H. Aita, L. Arrachea, and C. Naón, Physica B-Condensed Matter 407, 3158-3160 (2012).

9. M. Büttiker, Y. Imry, and Y. Azbel, Phys. Rev. A 30, 1982-1989 (1984).

10. M. Büttiker, Phys. Rev. Lett. 57, 1761-1764 (1986).

11. M. Büttiker, IBM J. Res. Dev. 32, 317-334 (1988).

12. S. Godoy and P. A. Mello, Europhys. Lett. 17, 243-248 (1992).

13. S. Godoy and P. A. Mello, Phys. Rev. B 46, 2346-2356 (1992).

14. V. A. Gopar, M. Martínez, and P. A. Mello, Phys. Rev. B 50, 2502-2509 (1994).

15. A. M. Song, A. Lorke, A. Kriele, J. P. Kotthaus, W. Wegscheider, and M. Bichler, Phys. Rev. Lett. 80, $3831-3834$ (1998).

16. A. M. Song, Phys. Rev. B 59, 9806-9809 (1999).

17. P. A. Mello, "Theory of Random Matrices: Spectral Statistics and Scattering Problems" in Mesoscopic Quantum Physics, edited by E. Akkermans, G. Montambaux, J.-L. Pichard and J. Zinn-Justinm, Elsevier, Amsterdam, 1995, pp. 435-492.

18. C. W. J. Beenakker, Rev. Mod. Phys. 69, 731-808 (1997).

19. Y. Alhassid, Rev. Mod. Phys. 72, 895-968 (2000).

20. F. J. Dyson, J. Math. Phys. 3, 140-156 (1962). 


\section{A|P| $\begin{aligned} & \text { Journal of } \\ & \text { Mathematical Physics }\end{aligned}$}

\section{Joint moments of proper delay times}

Angel M. Martínez-Argüello, Moisés Martínez-Mares, and Julio C. García

Citation: Journal of Mathematical Physics 55, 081901 (2014); doi: 10.1063/1.4890559

View online: http://dx.doi.org/10.1063/1.4890559

View Table of Contents: http://scitation.aip.org/content/aip/journal/jmp/55/8?ver=pdfcov

Published by the AIP Publishing

\section{Articles you may be interested in}

Moments of the transmission eigenvalues, proper delay times and random matrix theory II

J. Math. Phys. 53, 053504 (2012); 10.1063/1.4708623

Moments of the transmission eigenvalues, proper delay times, and random matrix theory. I

J. Math. Phys. 52, 103511 (2011); 10.1063/1.3644378

Fluctuations and Noise in Stochastic Spread of Respiratory Infection Epidemics in Social Networks AIP Conf. Proc. 665, 408 (2003); 10.1063/1.1584915

The long-time behavior of correlation functions in dynamical systems

AIP Conf. Proc. 502, 394 (2000); 10.1063/1.1302412

Periodicity versus chaos in certain population models

AIP Conf. Proc. 502, 215 (2000); 10.1063/1.1302388

\section{A|P| $\left.\right|_{\text {Applied Physics }} ^{\text {Journal of }}$}

Journal of Applied Physics is pleased to announce André Anders as its new Editor-in-Chief 


\title{
Joint moments of proper delay times
}

\author{
Angel M. Martínez-Argüello, ${ }^{1}$ Moisés Martínez-Mares, ${ }^{1}$ and Julio C. García ${ }^{2}$ \\ ${ }^{1}$ Departamento de Física, Universidad Autónoma Metropolitana-Iztapalapa, \\ Apartado Postal 55-534, 09340 México Distrito Federal, Mexico \\ ${ }^{2}$ Departamento de Matemáticas, Universidad Autónoma Metropolitana-Iztapalapa, \\ Apartado Postal 55-534, 09340 México Distrito Federal, Mexico
}

(Received 8 May 2014; accepted 7 July 2014; published online 24 July 2014)

We calculate negative moments of the $N$-dimensional Laguerre distribution for the orthogonal, unitary, and symplectic symmetries. These moments correspond to those of the proper delay times, which are needed to determine the statistical fluctuations of several transport properties through classically chaotic cavities, like quantum dots and microwave cavities with ideal coupling. () 2014 AIP Publishing LLC. [http://dx.doi.org/10.1063/1.4890559]

\section{INTRODUCTION}

The generalized Laguerre ensemble appears in the context of chaotic scattering ${ }^{1,2}$ as being the joint distribution of the reciprocals of the eigenvalues of the Wigner-Smith time delay matrix, known as the proper delay times.

The Wigner-Smith time delay matrix was introduced by $\mathrm{Smith}^{3}$ as a multi-channel generalization of the concept of delay time suffered by a wave packet, due to the interaction with a scattering potential, introduced by Wigner in the one channel situation. ${ }^{4}$ It is an $N \times N$ Hermitian matrix, where $N$ is the number of open modes (or channels), whose eigenvalues, the proper delay times, represent individual delay times on the channels. ${ }^{1,2}$ This time delay matrix is defined in terms of the energy derivative of the scattering matrix $S$, which is a fundamental entity in the description of scattering processes, and many transport properties in open systems, by relating the outgoing plane wave amplitudes to the incoming ones into the $N$ channels. ${ }^{5}$ Therefore, the Wigner-Smith time delay matrix is very important in the quantification of the transport properties which depend on the derivative of the scattering matrix with respect to the energy or an external parameter. The activity in this field has been increased due to the recent theoretical investigations ${ }^{6-9}$ that emerged from a measurement of a delay time in experiments of interaction of light with matter, with attosecond precision. ${ }^{10}$

In chaotic scattering, one of the most important questions is the effect of the chaotic classical dynamics of open ballistic cavities on the transport properties (see Refs. 11-14 and references therein). For example, the parametric derivative of the conductance through a quantum dot, which is the analogous to the level velocity in the characterization of a mesoscopic system, ${ }^{15-18}$ fluctuates with respect to some external parameters, that could be an applied magnetic field, ${ }^{19}$ the Fermi energy, ${ }^{20}$ or the dot shape,${ }^{21}$ when they are modified by a small amount. ${ }^{22-24}$ The DC current pumped through a quantum dot at zero bias is quantified by its fluctuations with respect to an applied magnetic field. ${ }^{25,26}$ The same situation occurs with the parametric derivative of the transmission coefficient, with respect to the frequency and cavity shape, in classical wave cavities. ${ }^{27,28}$ The statistical analysis needed for the quantification of a transport property, or its fluctuation, is very well realized by the random-matrix theory, that reveals the universal aspects of the problem. ${ }^{11-13}$ Since all of these quantities are defined in terms of the derivative of the scattering matrix this theory leads to quantify the transport properties, or their fluctuations, by the first moments of the proper delay times $\tau_{i}$ 's $(i=1, \ldots, N){ }^{24,27-29}$ In other cases, it is the distribution of the Wigner time delay, defined as the average of the proper delay times, what is of interest. For example, it is related to the dimensionless capacitance of a mesoscopic capacitor ${ }^{30,31}$ or to the thermopower, ${ }^{2}$ in disordered systems, it is used 
to characterize the classical diffusion in the metallic regime, ${ }^{32}$ the eigenfunction fluctuations (see Ref. 33 and references therein), and the metal-insulator transition. ${ }^{34,35}$

A lot of work concerning statistical studies of delay times and Wigner time delay has been developed in the last 30 years in several contexts of chaotic and disordered systems. ${ }^{2,31,36,37,39-43}$ For closed chaotic systems, those with non-perfect coupling to the $N$ open channels, the mean and variance of partial or phase-shift times, being the energy derivative of the eigenphases of the scattering matrix, as well as of the Wigner time delay, are well known in absence of time reversal symmetry $(\beta=2) .{ }^{36,37}$ The existing distribution for partial delay times for arbitrary $N$ are given in Refs. 37, 38, and 41 for $\beta=2$, and in Ref. 41 in the presence of time reversal symmetry $(\beta=1)$ and spin-rotation symmetry $(\beta=4)$. Besides, the distribution of the Wigner time delay was first calculated in Refs. 30 and 37 for the $N=1$ case, while the one for $N=2$ and $\beta=2$ was calculated, generalized, and verified to arbitrary $\beta$ in the ideal coupling case, and related to that of arbitrary coupling in Ref. 41. The joint distribution of the reciprocals of the proper delay times, which is the Laguerre distribution, is also known for arbitraries $N$ and $\beta .{ }^{1,2,41}$ However, it does not exist enough evaluations of the moments of this distribution for any symmetry and $N$. Expressions for the density of proper delay times and uncorrelated moments have been evaluated in Ref. 44 in the limit of very large $N$ and, for few and large number of channels and any symmetry, in Refs. 45 and 46 more recently, both cases for perfect coupling.

Our purpose in the present paper is to evaluate several joint moments of proper delay times for any symmetry, and determine their dependence with the number of channels, when this number is arbitrary. Many of these moments have not been calculated before, some of which have also importance on transport properties through ballistic open systems. Therefore, we regard perfect coupling to the $N$ open channels.

In Sec. II, we introduce the Laguerre distribution and establish the calculation of the moments we are interested in, in a general form, and summarize some known results for the partial times. In Sec. III, we present some definitions and properties that will help us to manage the Laguerre distribution, and allow us to determine the moments for arbitrary $N$ and any symmetry. Explicit calculations are performed in Sec. IV. We conclude in Sec. V.

\section{DELAY TIMES AND GENERALIZED LAGUERRE DISTRIBUTION}

\section{A. Proper delay times}

A symmetrized form of the Wigner-Smith time delay matrix ${ }^{1}$ can be written in dimensionless units as

$$
Q=-\frac{\mathrm{i} \hbar}{\tau_{H}} S^{-1 / 2} \frac{\partial S}{\partial E} S^{-1 / 2},
$$

where $E$ is the energy and $\tau_{H}$ is the Heisenberg time $\left(\tau_{H}=2 \pi \hbar / \Delta\right.$, with $\Delta$ the mean level spacing). The matrix $Q$ is an $N \times N$ Hermitian matrix, whose eigenvalues $q_{i}$ 's $(i=1, \ldots, N)$ are the proper delay times measured in units of $\tau_{H}$; that is, $q_{i}=\tau_{i} / \tau_{H}$. The distribution of the proper delay times is known and it is given in terms of their reciprocals. If $x_{i}=\tau_{H} / \tau_{i}$, the joint distribution of the $x_{i}$ 's is given by the Laguerre ensemble, namely, ${ }^{1}$

$$
P_{\beta}\left(x_{1}, \ldots, x_{N}\right)=C_{N}^{(\beta)} \prod_{a<b}^{N}\left|x_{b}-x_{a}\right|^{\beta} \prod_{c=1}^{N} x_{c}^{\beta N / 2} \mathrm{e}^{-\beta x_{c} / 2},
$$

where $\beta$ characterizes the universal statistics in Dyson's scheme: ${ }^{47} \beta=1$ in the presence of time reversal invariance (TRI) and integral spin or half-integral spin plus rotational symmetry, $\beta=4$ for TRI, half-integral spin and no rotational symmetry, and $\beta=2$ in the absence of TRI. ${ }^{5}$ In Eq. (2), $C_{N}^{(\beta)}$ is a normalization constant defined through the condition

$$
\int_{0}^{\infty} P_{\beta}\left(x_{1}, \ldots, x_{N}\right) \mathrm{d} x_{1} \cdots \mathrm{d} x_{N}=1 .
$$


Generalized moments of the proper delay times (in dimensionless units) like $\left\langle q_{1}^{k_{1}} \cdots q_{N}^{k_{N}}\right\rangle^{(\beta)}$ are just the negative generalized moments of the Laguerre ensemble. That is,

$$
\left\langle q_{1}^{k_{1}} \cdots q_{N}^{k_{N}}\right\rangle^{(\beta)}=\left\langle x_{1}^{-k_{1}} \cdots x_{N}^{-k_{N}}\right\rangle^{(\beta)}=\int_{0}^{\infty} \frac{P_{\beta}\left(x_{1}, \ldots, x_{N}\right)}{x_{1}^{k_{1}} \cdots x_{N}^{k_{N}}} \mathrm{~d} x_{1} \cdots \mathrm{d} x_{N},
$$

for $k_{j}(j=1, \ldots, N)$ an integer number.

\section{B. Partial delay times}

It is instructive to compare the distribution of the proper delay times with the distribution of "partial delay times," defined as the energy derivative of phase-shifts. The distribution of an individual partial delay time scaled with $\tau_{H}, \tau_{s}$, for the $\beta=2$ symmetry, is given by ${ }^{37,38}$

$$
P_{s}\left(\tau_{s}\right)=\frac{1}{N !} \tau_{s}^{-N-2} \mathrm{e}^{-1 / \tau_{s}} .
$$

In this case, it is easy to evaluate the $k$ th moment of the distribution, which is the following:

$$
\left\langle\tau_{s}^{k}\right\rangle=\frac{(N-k) !}{N !}
$$

for $k \leq N$. In particular

$$
\left\langle\tau_{s}\right\rangle=\frac{1}{N} \quad \text { and } \quad\left\langle\tau_{s}^{2}\right\rangle=\frac{1}{(N-1) N},
$$

which is the result expressed in Refs. 36 and 37.

\section{GENERAL EXPRESSIONS FOR THE JOINT MOMENTS}

To obtain a feasible general expression for the joint moments we notice that

$$
\prod_{a<b \leq N}\left(x_{b}-x_{a}\right)=\operatorname{det} V_{N}=\left|\begin{array}{ccccc}
1 & 1 & 1 & \cdots & 1 \\
x_{1} & x_{2} & x_{3} & \cdots & x_{N} \\
\vdots & \vdots & \vdots & \cdots & \vdots \\
x_{1}^{N-1} & x_{2}^{N-1} & x_{3}^{N-1} & \cdots & x_{N}^{N-1}
\end{array}\right|,
$$

which is known as the $N$ th-order Vandermonde determinant. ${ }^{48}$ It can be proved that ${ }^{49}$

$$
\operatorname{det} V_{N}=\sum_{\sigma} \operatorname{sgn} \sigma \prod_{a=1}^{N} x_{a}^{-1+\sigma(a)},
$$

where $\sigma$ is a permutation that belongs to the symmetric group of degree $N,{ }^{50}$

$$
S_{N}=\{\sigma:\{1, \ldots, N\} \rightarrow\{1, \ldots, N\} \mid \sigma \text { is a permutation }\}
$$

with $\operatorname{sgn} \sigma$ the signature of $\sigma$; the identity permutation is $\iota(a)=a$ for $a=1, \ldots, N$.

At this point is necessary to introduce some definitions and properties.

Definition 1. For $t_{a} \geq 0(a=1, \ldots, N)$, let

$$
\Delta_{N}=\left\{\left(t_{1}, \ldots, t_{N}\right): 0 \leq t_{1} \leq t_{2} \leq \cdots \leq t_{N}\right\} .
$$

Therefore, the Vandermonde determinant of Eq. (8) becomes positive for $\left(x_{1}, \ldots, x_{N}\right) \in \Delta_{N}$, so that

$$
\operatorname{det} V_{N}=\left|\operatorname{det} V_{N}\right|
$$


and the equality in Eq. (9) can be written as

$$
\left|\operatorname{det} V_{N}\right|^{\beta}=\sum_{\sigma_{1}, \ldots, \sigma_{\beta}} \prod_{i=1}^{\beta} \operatorname{sgn} \sigma_{i} \prod_{a=1}^{N} x_{a}^{-\beta+\sum_{j=1}^{\beta} \sigma_{j}(a)},
$$

where $\sigma_{j} \in S_{N}(j=1, \ldots, \beta)$.

Each permutation $\sigma \in S_{N}$ is associated to an unitary transformation $\pi_{\sigma}$ in the $N$-dimensional Euclidean space:

$$
\left(x_{1}, \ldots, x_{N}\right) \longrightarrow \pi_{\sigma}\left(x_{1}, \ldots, x_{N}\right)=\left[x_{\sigma(1)}, \ldots, x_{\sigma(N)}\right] .
$$

Definition 2. For any $\eta \in S_{N}$ and $t_{a} \geq 0(a=1, \ldots, N)$, let

$$
\Delta_{N}^{\eta}=\pi_{\eta}^{-1}\left(\Delta_{N}\right):=\left\{\left(t_{1}, \ldots, t_{N}\right): 0 \leq t_{\eta(1)} \leq t_{\eta(2)} \leq \cdots \leq t_{\eta(N)}\right\} .
$$

We notice that $\Delta_{N}^{\iota}=\Delta_{N}$; also, for any $\eta \in S_{N}$ and $\left(t_{1}, \ldots, t_{N}\right) \in \Delta_{N}^{\eta}$, from Eq. (14)

$$
\pi_{\eta}^{-1}\left(t_{1}, \ldots, t_{N}\right)=\left[t_{\eta^{-1}(1)}, \ldots, t_{\eta^{-1}(N)}\right] \in \Delta_{N}
$$

and therefore

$$
\operatorname{det} V_{N}\left[\pi_{\eta}^{-1}\left(t_{1}, \ldots, t_{N}\right)\right]=\prod_{a<b}\left[t_{\eta^{-1}(b)}-t_{\eta^{-1}(a)}\right] \geq 0,
$$

where Eq. (12) has been taken into account. Hence,

$$
\left|\operatorname{det} V_{N}\left(t_{1}, \ldots, t_{N}\right)\right|=\operatorname{det} V_{N}\left[\pi_{\eta}^{-1}\left(t_{1}, \ldots, t_{N}\right)\right] .
$$

Finally, for any non-negative measurable function $f\left(t_{1}, \ldots, t_{N}\right)$, the Change of Variables Theorem allows us to write

$$
\begin{aligned}
\int_{0}^{\infty} f\left(t_{1}, \ldots, t_{N}\right) \mathrm{d} t_{1} \cdots \mathrm{d} t_{N} & =\sum_{\eta \in S_{N}} \int_{\Delta_{N}^{\eta}} f\left(t_{1}, \ldots, t_{N}\right) \mathrm{d} t_{1} \cdots \mathrm{d} t_{N} \\
& =\sum_{\eta \in S_{N}} \int_{\Delta_{N}} f \circ \pi_{\eta}^{-1}\left(t_{1}, \ldots, t_{N}\right) \mathrm{d} t_{1} \cdots \mathrm{d} t_{N} .
\end{aligned}
$$

\section{A. Negative moments of the Laguerre distribution of $\boldsymbol{N}$ variables}

From the definition of the Vandermonde determinant (8) and Eq. (13), the Laguerre distribution of Eq. (2) can be written as

$$
P_{\beta}\left(x_{1}, \ldots, x_{N}\right)=C_{N}^{(\beta)} \sum_{\sigma_{1}, \ldots, \sigma_{\beta}} \prod_{i=1}^{\beta} \operatorname{sgn} \sigma_{i} \prod_{a=1}^{N} x_{a}^{\gamma_{a}} \mathrm{e}^{-\beta x_{a} / 2},
$$

where

$$
\gamma_{a}:=\gamma_{a}^{\sigma_{1}, \ldots, \sigma_{\beta}}=\frac{N \beta}{2}-\beta+\sum_{j=1}^{\beta} \sigma_{j}(a) \text { for } a=1, \ldots, N .
$$

It is convenient to write $P_{\beta}\left(x_{1}, \ldots, x_{N}\right)$ as

$$
P_{\beta}\left(x_{1}, \ldots, x_{N}\right)=C_{N}^{(\beta)} \sum_{\sigma_{1}, \ldots, \sigma_{\beta}} \prod_{i=1}^{\beta} \operatorname{sgn} \sigma_{i} \prod_{a=1}^{N}\left(\frac{2}{\beta}\right)^{\gamma_{a}+1} \Gamma\left(\gamma_{a}+1\right) f_{\gamma_{a}+1, \beta / 2}\left(x_{a}\right),
$$

where $f_{u, v}(t)$ is the probability density function of the Gamma distribution with parameters $u$ and $v,,^{51,52}$

$$
f_{u, v}(t)=\frac{v^{u} t^{u-1} \mathrm{e}^{-u t}}{\Gamma(u)}
$$

with $\Gamma(u)$ the Gamma function. ${ }^{52}$ It is important to notice that $\Gamma\left(\gamma_{a}+1\right)$ is well defined. 
For the joint moments we are interested in, we need the auxiliary function

$$
R_{\beta}\left(x_{1}, \ldots, x_{N}\right)=\frac{P_{\beta}\left(x_{1}, \ldots, x_{N}\right)}{x_{1}^{k_{1}} \cdots x_{N}^{k_{N}}},
$$

for $k_{a}(a=1, \ldots, N)$ an integer number. Using Eq. (22) this function can be written as

$$
R_{\beta}\left(x_{1}, \ldots, x_{N}\right)=C_{N}^{(\beta)} \sum_{\sigma_{1}, \ldots, \sigma_{\beta}} \prod_{i=1}^{\beta} \operatorname{sgn} \sigma_{i} \prod_{a=1}^{N}\left(\frac{2}{\beta}\right)^{\alpha_{a}} \Gamma\left(\alpha_{a}\right) f_{\alpha_{a}, \beta / 2}\left(x_{a}\right),
$$

where $\alpha_{a}=\gamma_{a}-k_{a}+1$ for $a=1, \ldots, N$, with $\gamma_{a}$ as in Eq. (21). Therefore, for any $\eta \in S_{N}$ and $\left(x_{1}, \ldots, x_{N}\right) \in \Delta_{N}^{\eta}$, the properties (16) and (18) allows us to write

$$
R_{\beta} \circ \pi_{\eta}^{-1}\left(x_{1}, \ldots, x_{N}\right)=\frac{P_{\beta}\left(x_{1}, \ldots, x_{N}\right)}{x_{\eta^{-1}(1)}^{k_{1}} \cdots x_{\eta^{-1}(N)}^{k_{N}}}=\frac{P_{\beta}\left(x_{1}, \ldots, x_{N}\right)}{x_{1}^{k_{\eta(1)}} \cdots x_{N}^{k_{\eta(N)}}} .
$$

The last equality is just the definition (24), which according to Eq. (25), it can be written as

$$
R_{\beta} \circ \pi_{\eta}^{-1}\left(x_{1}, \ldots, x_{N}\right)=C_{N}^{(\beta)} \sum_{\sigma_{1}, \ldots, \sigma_{\beta}} \prod_{i=1}^{\beta} \operatorname{sgn} \sigma_{i} \prod_{a=1}^{N}\left(\frac{2}{\beta}\right)^{\alpha_{a}^{\eta}} \Gamma\left(\alpha_{a}^{\eta}\right) f_{\alpha_{a}^{\eta}, \beta / 2}\left(x_{a}\right),
$$

where

$$
\alpha_{a}^{\eta}=\gamma_{a}-k_{\eta(a)}+1, \quad \text { for } a=1, \ldots, N .
$$

If we integrate this equation over $\Delta_{N}$ and sum over $\eta$, the property (19) allows us to arrive to the desired result, namely,

$$
\left\langle x_{1}^{-k_{1}} \cdots x_{N}^{-k_{N}}\right\rangle^{(\beta)}=C_{N}^{(\beta)} \sum_{\eta, \sigma_{1}, \ldots, \sigma_{\beta}} F_{\theta_{\eta, N}} \prod_{i=1}^{\beta} \operatorname{sgn} \sigma_{i} \prod_{a=1}^{N}\left(\frac{2}{\beta}\right)^{\alpha_{a}^{\eta}} \Gamma\left(\alpha_{a}^{\eta}\right),
$$

where $F_{\theta_{\eta, N}}$ is defined as

$$
F_{\theta_{\eta, N}}=\int_{\Delta_{N}} \prod_{a=1}^{N} f_{\alpha_{a}^{\eta}, \beta / 2}\left(x_{a}\right) \mathrm{d} x_{a}
$$

with

$$
\theta_{\eta, N}:=\theta_{\eta, N}^{\sigma_{1}, \ldots, \sigma_{\beta}}:=(\alpha_{1}^{\eta}, \ldots, \alpha_{N}^{\eta}, \underbrace{\beta / 2, \ldots, \beta / 2}_{N}) .
$$

It is clear that the normalization constant $C_{N}^{(\beta)}$ can be obtained from Eq. (29) for $k_{a}=0$ ( $a=1$, $\ldots, N)$, in which case the argument in the sum of Eq. (29) is independent of $\eta$, such that the sum over $\eta$ is exactly $N$ !. It is given by

$$
C_{N}^{(\beta)}=\left[N ! \sum_{\sigma_{1}, \ldots, \sigma_{\beta}} F_{\theta_{N}} \prod_{i=1}^{\beta} \operatorname{sgn} \sigma_{i} \prod_{a=1}^{N}\left(\frac{2}{\beta}\right)^{\gamma_{a}+1} \Gamma\left(\gamma_{a}+1\right)\right]^{-1},
$$

where

$$
F_{\theta_{N}}=\int_{\Delta_{N}} \prod_{a=1}^{N} f_{\gamma_{a}+1, \beta / 2}\left(x_{a}\right) \mathrm{d} x_{a} .
$$

Two Remarks are worth mentioning.

Remark 1. In Eqs. (29) and (32), it is necessary to take into account the dependence of the parameters $\theta_{N}$ and $\theta_{\eta, N}$ on the permutations $\sigma_{1}, \ldots, \sigma_{\beta}$ but we omitted to write it explicitly, for simplicity. 
Remark 2. Since the minimum possible value of any permutation is 1 , it is easy to show that $\gamma_{a}-k_{a}+1>0$ for $0 \leq k_{a}<\frac{N \beta}{2}+1$.

This Remark ensures that the Gamma function that appears in Eq. (29) is well defined. It is important to notice that the property expressed in Remark 2 is a general result that restricts the values of $k_{a}(a=1, \ldots, N)$. This condition is the most general and coincides with that of Ref. 45 for the particular case when $k_{a}=k$, for $a=1, \ldots, N$. The restriction of $k$ in Eq. (6) appears also as a particular case.

\section{1. $\beta$ an even integer number}

When $\beta$ is an even positive integer then $\left|\operatorname{det} V_{N}\right|^{\beta}=\left(\operatorname{det} V_{N}\right)^{\beta}$ for any $\left(x_{1}, \ldots, x_{N}\right) \in \mathbb{R}^{N}$, being $\mathbb{R}^{N}$ the real $N$-dimensional Euclidean space. In this case there is no need to use Eq. (19) so, in the computations of the negative moments, we obtain simpler formulas for the joint negative moments of the delay times.

If we integrate Eq. (25), we have that

$$
\begin{aligned}
\left\langle x_{1}^{-k_{1}} \cdots x_{N}^{-k_{N}}\right\rangle^{(\beta)} & =C_{N}^{(\beta)}\left(\frac{2}{\beta}\right)^{N} \sum_{\sigma_{1}, \ldots, \sigma_{\beta}} \prod_{i=1}^{\beta} \operatorname{sgn} \sigma_{i} \prod_{a=1}^{N} \int_{0}^{\infty}\left(\frac{\beta}{2}\right) x^{\gamma_{a}-k_{a}} \mathrm{e}^{-\beta x / 2} \mathrm{~d} x \\
& =C_{N}^{(\beta)}\left(\frac{2}{\beta}\right)^{N} A_{N}^{(\beta)}\left(k_{1}, \ldots, k_{N}\right),
\end{aligned}
$$

where

$$
A_{N}^{(\beta)}\left(k_{1}, \ldots, k_{N}\right)=\sum_{\sigma_{1}, \ldots, \sigma_{\beta}} \prod_{i=1}^{\beta} \operatorname{sgn} \sigma_{i} \prod_{a=1}^{N}\left(\frac{2}{\beta}\right)^{\gamma_{a}-k_{a}} \Gamma\left(\gamma_{a}-k_{a}+1\right) .
$$

If $k_{1}=\cdots=k_{N}=0$, then Eq. (34) implies that $C_{N}^{(\beta)}=(\beta / 2)^{N} / A_{N}^{(\beta)}$, with $A_{N}^{(\beta)}=A_{N}^{(\beta)}(0, \ldots, 0)$.

\section{EXPLICIT COMPUTATIONS}

We present explicit calculations with $N=1$ and 2 , for $\beta=1$ and 4 , and $N=1,2,3$ and 4 for $\beta=2$. We make explicit, as far as possible, the dependence on the corresponding permutations. We start our calculations with the $\beta=1$ case since it is usually the most difficult to treat analytically.

\section{A. Explicit calculations for $\beta=1$}

For the particular case of $\beta=1$ but arbitrary $N$, Eq. (21) is written as

$$
\gamma_{a}:=\gamma_{a}^{\sigma_{1}}=\frac{N}{2}-1+\sigma_{1}(a), \text { for } a=1, \ldots, N,
$$

such that the moments given by Eq. (29) are simplified to

$$
\left\langle x_{1}^{-k_{1}} \cdots x_{N}^{-k_{N}}\right\rangle^{(1)}=C_{N}^{(1)} \sum_{\eta, \sigma_{1}} F_{\theta_{\eta, N}} \operatorname{sgn} \sigma_{1} \prod_{a=1}^{N} 2^{\sigma_{1}(a)-k_{\eta(a)}+N / 2} \Gamma\left[\sigma_{1}(a)-k_{\eta(a)}+N / 2\right],
$$

where $0 \leq k_{a}<1+N / 2$.

\section{The $N=1$ case}

According to Eq. (10), $S_{1}=\{\iota(1)=1\}$, that is, only the identity belongs to the symmetric group $S_{1}$, such that $\eta(1)=\sigma_{1}(1)=\iota(1)=1$ and $\theta_{\eta, 1}=\theta_{1}=(3 / 2-k, 1 / 2)$. Therefore, from Eq. (37),

$$
\left\langle x^{-k}\right\rangle^{(1)}=C_{1}^{(1)} F_{\theta_{1}} 2^{-k+3 / 2} \Gamma(-k+3 / 2)=C_{1}^{(1)} 2^{-k+3 / 2} \Gamma(-k+3 / 2),
$$


where we have used that

$$
F_{\theta_{1}}=\frac{1}{2^{3 / 2} \Gamma(3 / 2)} \int_{0}^{\infty} t^{1 / 2} \mathrm{e}^{-t} \mathrm{~d} t=1 .
$$

The only values that $k$ can take are 0 and 1 . For $k=0$, we obtain the normalization constant: $C_{1}^{(1)}=1 / 2^{3 / 2}(1 / 2)$ !, while for $k=1$ we have

$$
\left\langle x_{1}^{-k}\right\rangle^{(1)}=\left(\frac{1}{2}\right)^{k} \frac{\left(\frac{1}{2}-k\right) !}{\left(\frac{1}{2}\right) !} .
$$

\section{The $N=2$ case}

In this case, $S_{2}=\{\iota, \sigma\}$, such that $\sigma_{1}=\iota, \sigma$ with $\iota(a)=a(a=1,2), \sigma(1)=2$ and $\sigma(2)=1$; therefore, $\gamma_{a}=\sigma_{1}(a)$. Equation (37) for $N=2$ gives

$$
\begin{aligned}
\left\langle x_{1}^{-k_{1}} x_{2}^{-k_{2}}\right\rangle^{(1)}= & C_{2}^{(1)} 2^{5-k_{1}-k_{2}}\left[\left(F_{\theta_{l, 2}^{\iota}}-F_{\theta_{\sigma, 2}^{\sigma}}\right) \Gamma\left(2-k_{1}\right) \Gamma\left(3-k_{2}\right)\right. \\
& \left.+\left(F_{\theta_{\sigma, 2}^{l}}-F_{\theta_{t, 2}^{\sigma}}\right) \Gamma\left(3-k_{1}\right) \Gamma\left(2-k_{2}\right)\right] .
\end{aligned}
$$

Here, Eq. (28) says that $\alpha_{a}^{\eta}=\sigma_{1}(a)-k_{\eta(a)}+1$ and Eq. (31) gives

$$
\theta_{\eta, N}^{\sigma_{1}}=\left[\sigma_{1}(i)-k_{\eta(1)}+1, \sigma_{1}(2)-k_{\eta(2)}+1,1 / 2,1 / 2\right] .
$$

According to Eq. (A12) of the Appendix, we can determine the coefficients $F_{\theta_{\eta, N}}^{\sigma_{1}}$ by means of the negative binomial distribution $N B_{\alpha_{2}, p_{2}}$ with parameters $\alpha_{2}$ and $p_{2}=1 / 2 .{ }^{53}$ For the particular case of $N=2$

$$
F_{\theta_{\eta, 2}}^{\sigma_{1}}=1-\sum_{\ell=0}^{\sigma_{1}(1)-k_{\eta(1)}} N B_{\sigma_{1}(2)-k_{\eta(2)}+1,1 / 2}(\ell)
$$

for any $\eta$ and $\sigma_{1} \in S_{2}$ and $k_{a}=0,1$.

For the particular case of $k_{1}=k_{2}=0$ we have that

$$
\begin{aligned}
& F_{\theta_{2}^{\ell}}=1-\sum_{\ell=0}^{\iota(1)} N B_{\ell(2)+1,1 / 2}(\ell)=1-\sum_{\ell=0}^{1} N B_{3,1 / 2}(\ell)=\frac{11}{16}, \\
& F_{\theta_{2}^{\sigma}}=1-\sum_{\ell=0}^{\sigma(1)} N B_{\sigma(2)+1,1 / 2}(\ell)=1-\sum_{\ell=0}^{2} N B_{2,1 / 2}(\ell)=\frac{5}{16},
\end{aligned}
$$

where we have used the Definition (A4), and the normalization constant is $C_{2}^{(1)}=1 / 48$.

In very similar way, for $k_{1}=k=1$ and $k_{2}=0$,

$$
F_{\theta_{t, 2}}=\frac{7}{8}, \quad F_{\theta_{\sigma, 2}^{\iota}}=\frac{1}{2}, \quad F_{\theta_{t, 2}^{\sigma}}=\frac{1}{2}, \quad \text { and } \quad F_{\theta_{\sigma, 2}^{\sigma}}=\frac{1}{8}
$$

such that

$$
\left\langle x_{1}^{-k}\right\rangle^{(1)}=\left(\frac{1}{2}\right)^{k} \frac{(1-k) !}{1 !} K_{2}^{(1)}(k, 0)
$$

where

$$
K_{2}^{(1)}(k, 0)=1 \quad \text { for } \quad k=1 .
$$

For $k_{1}=k_{2}=k=1$, we have that

$$
F_{\theta_{t, 2}^{\iota}}=\frac{3}{4}, \quad F_{\theta_{\sigma, 2}^{\iota}}=\frac{3}{4}, \quad F_{\theta_{t, 2}^{\sigma}}=\frac{1}{4}, \quad \text { and } \quad F_{\theta_{\sigma, 2}^{\sigma}}=\frac{1}{4},
$$


and we can write the corresponding moment as

$$
\left\langle x_{1}^{-k} x_{2}^{-k}\right\rangle^{(1)}=\left(\frac{1}{2}\right)^{2 k} \frac{(1-k) !}{1 !} \frac{\left(\frac{3}{2}-k\right) !}{\frac{3}{2} !} .
$$

For $\beta=1$, the calculations become much more complicated for values of $N$ larger than 2 . However, some moments can be obtained numerically, some of which are shown in Sec. IV D.

\section{B. Explicit calculations for $\beta=2$}

From Eq. (21), we have that $\gamma_{a}=N-2+\sigma_{1}(a)+\sigma_{2}(a)(a=1, \ldots, N)$ and Eq. (35) is written as

$$
A_{N}^{(2)}\left(k_{1}, \ldots, k_{N}\right)=\sum_{\sigma_{1}, \sigma_{2} \in S_{N}} \operatorname{sgn}\left(\sigma_{1} \sigma_{2}\right) \prod_{a=1}^{N}\left[N-2+\sigma_{1}(a)+\sigma_{2}(a)-k_{a}\right] ! ;
$$

the normalization constant is $C_{N}^{(2)}=1 / A_{N}^{(2)}$, with $A_{N}^{(2)}=A_{N}^{(2)}(0, \ldots, 0)$, and the moments are given by Eq. (34).

\section{The $N=1$ case}

In this special case, $\gamma_{1}=\sigma_{1}+\sigma_{2}-1$, such that Eq. (51) reads

$$
A_{1}^{(2)}(k)=\sum_{\sigma_{1}, \sigma_{2} \in S_{1}} \operatorname{sgn}\left(\sigma_{1} \sigma_{2}\right)\left(\sigma_{1}+\sigma_{2}-k-1\right) !=(1-k) !
$$

Since $N \beta / 2+1=2$, according to Remark 2 the maximum value for $k$ is 1 . The normalization constant is obtained for $k=0$ as $C_{1}^{(2)}=1 / 1$ !. Therefore, the only moment is

$$
\left\langle x_{1}^{-k}\right\rangle^{(2)}=\frac{(1-k) !}{1 !} \text { for } k=1 .
$$

\section{The $N=2$ case}

In this case, we observe that $\gamma_{a}=\sigma_{1}(a)+\sigma_{2}(a)(a=1,2)$ and Eq. (51) becomes

$$
A_{2}^{(2)}\left(k_{1}, k_{2}\right)=\left(2-k_{1}\right) !\left(4-k_{2}\right) !+\left(4-k_{1}\right) !\left(2-k_{2}\right) !-2\left(3-k_{1}\right) !\left(3-k_{2}\right) ! .
$$

Here, $N \beta / 2+1=3$ such that the maximum order of the negative moments is 2 ; that is, $k_{a}=0,1,2$ $(a=1,2)$.

For $k_{1}=k_{2}=0$ we obtain the normalization constant, $C_{2}^{(2)}=1 / 2 !(3 ! \cdot 2 ! \cdot 1$ !). The corresponding moments for $k_{1}=k$ and $k_{2}=0$ are

$$
\left\langle x_{1}^{-k}\right\rangle^{(2)}=\frac{(2-k) !}{2 !} K_{2}^{(2)}(k, 0),
$$

where

$$
K_{2}^{(2)}(k, 0)=\frac{1}{3 !}[12-6(3-k)+(3-k)(4-k)] .
$$

Also, for $k_{1}=k_{2}=k$ we have

$$
\left\langle x_{1}^{-k} x_{2}^{-k}\right\rangle^{(2)}=\frac{(3-k) !}{3 !} \frac{(2-k) !}{2 !} .
$$

The remaining joint negative moment is

$$
\left\langle x_{1}^{-2} x_{2}^{-1}\right\rangle^{(2)}=\frac{1 !}{3 !} .
$$




\section{The $\mathbf{N}=3$ case}

For $N=3, \gamma_{a}=\sigma_{1}(a)+\sigma_{2}(a)+1(a=1,2,3)$ and Eq. (51) becomes

$$
\begin{aligned}
A_{3}^{(2)}\left(k_{1}, k_{2}, k_{3}\right)= & \left(3-k_{1}\right) !\left(5-k_{2}\right) !\left(7-k_{3}\right) !+\left(3-k_{1}\right) !\left(7-k_{2}\right) !\left(5-k_{3}\right) ! \\
+ & \left(5-k_{1}\right) !\left(3-k_{2}\right) !\left(7-k_{3}\right) !+\left(5-k_{1}\right) !\left(7-k_{2}\right) !\left(3-k_{3}\right) ! \\
+ & \left(7-k_{1}\right) !\left(3-k_{2}\right) !\left(5-k_{3}\right) !+\left(7-k_{1}\right) !\left(5-k_{2}\right) !\left(3-k_{3}\right) ! \\
& -2\left(3-k_{1}\right) !\left(6-k_{2}\right) !\left(6-k_{3}\right) !-2\left(6-k_{1}\right) !\left(3-k_{2}\right) !\left(6-k_{3}\right) ! \\
& -2\left(6-k_{1}\right) !\left(6-k_{2}\right) !\left(3-k_{3}\right) !-2\left(4-k_{1}\right) !\left(4-k_{2}\right) !\left(7-k_{3}\right) ! \\
& -2\left(4-k_{1}\right) !\left(7-k_{2}\right) !\left(4-k_{3}\right) !-2\left(7-k_{1}\right) !\left(4-k_{2}\right) !\left(4-k_{3}\right) ! \\
+ & 2\left(4-k_{1}\right) !\left(5-k_{2}\right) !\left(6-k_{3}\right) !+2\left(4-k_{1}\right) !\left(6-k_{2}\right) !\left(5-k_{3}\right) ! \\
+ & 2\left(6-k_{1}\right) !\left(5-k_{2}\right) !\left(4-k_{3}\right) !+2\left(5-k_{1}\right) !\left(4-k_{2}\right) !\left(6-k_{3}\right) ! \\
+ & 2\left(5-k_{1}\right) !\left(6-k_{2}\right) !\left(4-k_{3}\right) !+2\left(6-k_{1}\right) !\left(4-k_{2}\right) !\left(5-k_{3}\right) ! \\
& -6\left(5-k_{1}\right) !\left(5-k_{2}\right) !\left(5-k_{3}\right) ! .
\end{aligned}
$$

The maximum value of $k_{a}(a=1,2,3)$ is 3 . If we evaluate this expression at $k_{1}=k_{2}=k_{3}=0$ we obtain $C_{3}^{(2)}=1 / 3 !(5 ! \cdot 4 ! \cdot 3 ! \cdot 2 ! \cdot 1 !)$.

The result for the moments for $k_{1}=k$ and $k_{2}=k_{3}=0$ is given by

$$
\left\langle x_{1}^{-k}\right\rangle^{(2)}=\frac{(3-k) !}{3 !} K_{3}^{(2)}(k, 0,0),
$$

where

$$
\begin{aligned}
K_{3}^{(2)}(k, 0,0) & =\frac{1}{6 !}[5 \cdot 6 !-4 \cdot 6 !(4-k)+150 \cdot 3 !(4-k)(5-k) \\
& -5 !(4-k)(5-k)(6-k)+3 !(4-k)(5-k)(6-k)(7-k)] .
\end{aligned}
$$

In similar way, for $k_{1}=k_{2}=k$ and $k_{3}=0$ we have that

$$
\left\langle x_{1}^{-k} x_{2}^{-k}\right\rangle^{(2)}=\frac{(3-k) !}{3 !} \frac{(4-k) !}{4 !} K_{3}^{(2)}(k, k, 0),
$$

where

$$
\begin{aligned}
K_{3}^{(2)}(k, k, 0) & =\frac{1}{6 !}[7 !-6 !(k-1)(5-k) \\
& \left.-4 \cdot 4 !(4-k)(5-k)(6-k)+3 !(4-k)(5-k)^{2}(6-k)\right] .
\end{aligned}
$$

The moments for $k_{1}=k_{2}=k_{3}=k$ are given by

$$
\left\langle x_{1}^{-k} x_{2}^{-k} x_{3}^{-k}\right\rangle^{(2)}=\frac{(5-k) !}{5 !} \frac{(4-k) !}{4 !} \frac{(3-k) !}{3 !} .
$$

The remaining terms can be evaluated directly from Eq. (59). They are the following:

$$
\left\langle x_{1}^{-2} x_{2}^{-1}\right\rangle^{(2)}=\frac{3 !}{5 !}, \quad\left\langle x_{1}^{-3} x_{2}^{-1}\right\rangle^{(2)}=\frac{62}{6 !}, \quad \text { and } \quad\left\langle x_{1}^{-3} x_{2}^{-2}\right\rangle^{(2)}=\frac{3}{5 !} ;
$$

also,

$$
\begin{gathered}
\left\langle x_{1}^{-2} x_{2}^{-1} x_{3}^{-1}\right\rangle^{(2)}=\frac{6}{6 !},\left\langle x_{1}^{-2} x_{2}^{-2} x_{3}^{-1}\right\rangle^{(2)}=\frac{2 !}{6 !},\left\langle x_{1}^{-3} x_{2}^{-1} x_{3}^{-1}\right\rangle^{(2)}=\frac{10}{6 !},\left\langle x_{1}^{-3} x_{2}^{-2} x_{3}^{-1}\right\rangle^{(2)}=\frac{5}{2 \cdot 6 !} \\
\left\langle x_{1}^{-3} x_{2}^{-2} x_{3}^{-2}\right\rangle^{(2)}=\frac{1}{2 \cdot 6 !}, \quad\left\langle x_{1}^{-3} x_{2}^{-3} x_{3}^{-1}\right\rangle^{(2)}=\frac{1}{4 \cdot 5 !}, \quad \text { and } \quad\left\langle x_{1}^{-3} x_{2}^{-3} x_{3}^{-2}\right\rangle^{(2)}=\frac{1}{4 \cdot 6 !}
\end{gathered}
$$




\section{The $N=4$ case}

From Eq. (21), $\gamma_{a}=\sigma_{1}(a)+\sigma_{2}(a)+2(a=1,2,3,4)$; in this case Eq (51) gives

$$
\begin{aligned}
A_{4}^{(2)}\left(k_{1}, k_{2}, k_{3}, k_{4}\right) & =\left(4-k_{1}\right) !\left(6-k_{2}\right) !\left(8-k_{3}\right) !\left(10-k_{4}\right) !-\left(4-k_{1}\right) !\left(6-k_{2}\right) !\left(9-k_{3}\right) !\left(9-k_{4}\right) ! \\
& -\left(4-k_{1}\right) !\left(7-k_{2}\right) !\left(7-k_{3}\right) !\left(10-k_{4}\right) !+\left(4-k_{1}\right) !\left(7-k_{2}\right) !\left(9-k_{3}\right) !\left(8-k_{4}\right) ! \\
& +\left(4-k_{1}\right) !\left(8-k_{2}\right) !\left(7-k_{3}\right) !\left(9-k_{4}\right) !-\left(4-k_{1}\right) !\left(8-k_{2}\right) !\left(8-k_{3}\right) !\left(8-k_{4}\right) ! \\
& -\left(5-k_{1}\right) !\left(5-k_{2}\right) !\left(8-k_{3}\right) !\left(10-k_{4}\right) !+\left(5-k_{1}\right) !\left(5-k_{2}\right) !\left(9-k_{3}\right) !\left(9-k_{4}\right) ! \\
& +\left(5-k_{1}\right) !\left(7-k_{2}\right) !\left(6-k_{3}\right) !\left(10-k_{4}\right) !-\left(5-k_{1}\right) !\left(7-k_{2}\right) !\left(9-k_{3}\right) !\left(7-k_{4}\right) ! \\
& -\left(5-k_{1}\right) !\left(8-k_{2}\right) !\left(6-k_{3}\right) !\left(9-k_{4}\right) !+\left(5-k_{1}\right) !\left(8-k_{2}\right) !\left(8-k_{3}\right) !\left(7-k_{4}\right) ! \\
& +\left(6-k_{1}\right) !\left(5-k_{2}\right) !\left(7-k_{3}\right) !\left(10-k_{4}\right) !-\left(6-k_{1}\right) !\left(5-k_{2}\right) !\left(9-k_{3}\right) !\left(8-k_{4}\right) ! \\
& -\left(6-k_{1}\right) !\left(6-k_{2}\right) !\left(6-k_{3}\right) !\left(10-k_{4}\right) !+\left(6-k_{1}\right) !\left(6-k_{2}\right) !\left(9-k_{3}\right) !\left(7-k_{4}\right) ! \\
& +\left(6-k_{1}\right) !\left(8-k_{2}\right) !\left(6-k_{3}\right) !\left(8-k_{4}\right) !-\left(6-k_{1}\right) !\left(8-k_{2}\right) !\left(7-k_{3}\right) !\left(7-k_{4}\right) ! \\
& -\left(7-k_{1}\right) !\left(5-k_{2}\right) !\left(7-k_{3}\right) !\left(9-k_{4}\right) !+\left(7-k_{1}\right) !\left(5-k_{2}\right) !\left(8-k_{3}\right) !\left(8-k_{4}\right) ! \\
& +\left(7-k_{1}\right) !\left(6-k_{2}\right) !\left(6-k_{3}\right) !\left(9-k_{4}\right) !-\left(7-k_{1}\right) !\left(6-k_{2}\right) !\left(8-k_{3}\right) !\left(7-k_{4}\right) ! \\
& -\left(7-k_{1}\right) !\left(7-k_{2}\right) !\left(6-k_{3}\right) !\left(8-k_{4}\right) !+\left(7-k_{1}\right) !\left(7-k_{2}\right) !\left(7-k_{3}\right) !\left(7-k_{4}\right) ! \\
& +\left(\text { permutations of } k_{1}, k_{2}, k_{3}, k_{4}\right) .
\end{aligned}
$$

This equation is well defined because the maximum allowed value for $k_{a}(a=1,2,3,4)$ is 4 .

Although the calculation of $A_{4}^{(2)}\left(k_{1}, k_{2}, k_{3}, k_{4}\right)$ for arbitrary set of values of the $k_{a}$ 's is not difficult, it consists of many terms that are not easy to follow. Two quantities are clearly feasible: one for $k_{a}=0$ and the other for $k_{a}=k$. This is due to the fact that the permutations of $k_{a}$ 's in Eq. (68) give the same terms that have been explicitly written. The first quantity gives the normalization constant, $C_{4}^{(2)}=1 / 4 !(7 ! \cdot 6 ! \cdot 5 ! \cdot 4 ! \cdot 3 ! \cdot 2 ! \cdot 1 !)$. The second quantity is the moment

$$
\left\langle x_{1}^{-k} x_{2}^{-k} x_{3}^{-k} x_{4}^{-k}\right\rangle^{(2)}=\frac{(7-k) !}{7 !} \frac{(6-k) !}{6 !} \frac{(5-k) !}{5 !} \frac{(4-k) !}{4 !} .
$$

Any other moment is difficult to compute arithmetically, with great effort we arrive to the following results:

$$
\begin{gathered}
\left\langle x_{1}^{-1}\right\rangle^{(2)}=\frac{3 !}{4 !}, \quad\left\langle x_{1}^{-2}\right\rangle^{(2)}=\frac{8 \cdot 2 !}{5 !}, \quad\left\langle x_{1}^{-3}\right\rangle^{(2)}=\frac{16 \cdot 3 !}{6 !}, \quad \text { and } \quad\left\langle x_{1}^{-4}\right\rangle^{(2)}=\frac{59 \cdot 4 !}{7 !} \\
\left\langle x_{1}^{-1} x_{2}^{-1}\right\rangle^{(2)}=\frac{6}{5 !}, \quad\left\langle x_{1}^{-2} x_{2}^{-2}\right\rangle^{(2)}=\frac{3}{6 !}\left(\frac{8 \cdot 9}{6 \cdot 7}\right), \quad \text { and } \quad\left\langle x_{1}^{-3} x_{2}^{-3}\right\rangle^{(2)}=\frac{8 \cdot 5 !}{9 !} ; \\
\left\langle x_{1}^{-1} x_{2}^{-1} x_{3}^{-1}\right\rangle^{(2)}=\frac{1}{5 !}, \quad\left\langle x_{1}^{-2} x_{2}^{-2} x_{3}^{-2}\right\rangle^{(2)}=\frac{1}{7 !}, \quad \text { and } \quad\left\langle x_{1}^{-3} x_{2}^{-3} x_{3}^{-3}\right\rangle^{(2)}=\frac{51}{10 !} .
\end{gathered}
$$

\section{Explicit calculations for $\beta=4$}

For $\beta=4$, Eq. (21) says that $\gamma_{a}=2 N-4+\sigma_{1}(a)+\sigma_{2}(a)+\sigma_{3}(a)+\sigma_{4}(a)(a=1, \ldots$, $N)$, such that Eq. (35) gives

$$
A_{N}^{(4)}\left(k_{1}, \ldots, k_{N}\right)=\sum_{\sigma_{1}, \sigma_{2}, \sigma_{3} \sigma_{4} \in S_{N}} \operatorname{sgn}\left(\sigma_{1} \sigma_{2} \sigma_{3} \sigma_{4}\right) \prod_{i=1}^{N} \frac{\left(\gamma_{i}-k_{i}\right) !}{2^{\gamma_{i}-k_{i}}} .
$$

The normalization constant is given by $C_{N}^{(4)}=2^{N} / A_{N}^{(4)}$ and the moments are given by Eq. (34). 


\section{The $N=1$ case}

In this case, $\gamma=\left(\sigma_{1}+\sigma_{2}+\sigma_{3}+\sigma_{4}-k-2\right)$ !. Equation (73) can be written as

$$
A_{1}^{(4)}(k)=2^{k} \frac{(2-k) !}{2^{2}}
$$

such that $A_{1}^{(4)}=1 / 2$ and $C_{1}^{(4)}=2^{2}$. Therefore,

$$
\left\langle x_{1}^{-k}\right\rangle^{(4)}=2^{k} \frac{(2-k) !}{2 !} \text { for } k=1,2 .
$$

\section{The $N=2$ case}

Here, $\sigma_{i} \in S_{2}(i=1,2,3,4)$ and

$$
\begin{aligned}
A_{2}^{(4)}\left(k_{1}, k_{2}\right) & =2^{k_{1}+k_{2}-12}\left[\left(4-k_{1}\right) !\left(8-k_{2}\right) !-4\left(5-k_{1}\right) !\left(7-k_{2}\right) !+3\left(6-k_{1}\right) !\left(6-k_{2}\right) !\right] \\
& +\left(\text { permutations of } k_{1} \text { and } k_{2}\right) .
\end{aligned}
$$

Taking $k_{1}=k_{2}=0$, we have that $C_{2}^{(4)}=2^{14} / 3 !\left(5 ! \cdot 4 ! \cdot 3 ! \cdot 2 ! \cdot 1\right.$ !), while for $k_{1}=k_{2}=k$,

$$
\left\langle x_{1}^{-k} x_{2}^{-k}\right\rangle^{(4)}=2^{2 k} \frac{(6-k) !}{6 !} \frac{(4-k) !}{4 !}, \quad \text { for } k=1,2,3,4 .
$$

Also, for $k_{1}=k$ and $k_{2}=0$, we get

$$
\left\langle x_{1}^{-k}\right\rangle^{(4)}=2^{k} \frac{(4-k) !}{4 !} K_{2}^{(4)}(k, 0),
$$

where

$$
K_{2}^{(4)}(k, 0)=\frac{1}{6 ! \cdot 3 ! \cdot 2 !}[8 !+2 \cdot 6 !(5-k)(4-3 k)-4 !(12+k)(7-k)(6-k)(5-k)] .
$$

From Eq. (76) it is easy to see that the remaining moments are given by

$$
\begin{aligned}
& \left\langle x_{1}^{-2} x_{2}^{-1}\right\rangle^{(4)}=\frac{1}{9}, \quad\left\langle x_{1}^{-3} x_{2}^{-1}\right\rangle^{(4)}=\frac{7}{45}, \quad \text { and } \quad\left\langle x_{1}^{-3} x_{2}^{-2}\right\rangle^{(4)}=\frac{2}{45} \\
& \left\langle x_{1}^{-4} x_{2}^{-1}\right\rangle^{(4)}=\frac{22}{45}, \quad\left\langle x_{1}^{-4} x_{2}^{-2}\right\rangle^{(4)}=\frac{2}{15}, \quad \text { and } \quad\left\langle x_{1}^{-4} x_{2}^{-3}\right\rangle^{(4)}=\frac{2}{45} .
\end{aligned}
$$

\section{Joint moments of proper delay times for arbitraries $N$ and $\beta$}

The set of equations (40), (47), (53), (55), (60), (75), and (78); (50), (57), (62), and (77); and (64) and (69) suggest a general expression for the joint moments for any symmetry and number of channels, which is

$$
\left\langle q_{1}^{k} \cdots q_{m}^{k}\right\rangle^{(\beta)}=\left[\prod_{n=N}^{N+m-1}\left(\frac{\beta}{2}\right)^{k} \frac{\left(\frac{\beta n}{2}-k\right) !}{\left(\frac{\beta n}{2}\right) !}\right] K_{N}^{(\beta)}(\underbrace{k, \ldots, k}_{m}, \underbrace{0, \ldots, 0}_{N-m}) \text { for } m \leq N,
$$

where $K_{N}^{(\beta)}(\underbrace{k, \ldots, k}_{m}, \underbrace{0, \ldots, 0}_{N-m})$ has a particular expression for each values of $N$ and $m$, as can be seen in Eqs. (48), (56), (61), (63), and (79). A closed expression for this quantity is difficult to obtain analytically, but it reduces to 1 for $m=N$, as is suggested also by Eqs. (40), (50), (53), (57), (64), (69), (75), and (77),

$$
K_{N}^{(\beta)}(k, \ldots, k)=1,
$$

as well as for $k=1$ and $m \leq N$

$$
K_{N}^{(\beta)}(\underbrace{1, \ldots, 1}_{m}, \underbrace{0, \ldots, 0}_{N-m})=1,
$$

as can be seen by the direct evaluation of the equations just mentioned above. 
A particular case of interest is the $k$ th moment for $m=1$ (single variable); we find that

$$
\left\langle q_{1}^{k}\right\rangle^{(\beta)}=\left(\frac{\beta}{2}\right)^{k} \frac{\left(\frac{\beta N}{2}-k\right) !}{\left(\frac{\beta N}{2}\right) !} K_{N}^{(\beta)}(k, 0, \ldots, 0), \quad \text { for } \quad k<\frac{\beta N}{2}+1
$$

What is interesting of this result is that the $k$ th moment of a proper delay time differs from that of a partial delay time, as can be seen if we compare this result for $\beta=2$ with Eq. (6) ${ }^{36,37}$ Moreover, it allows us to generalize the $k$ th moment for a partial time for any $\beta$ and $N$, namely,

$$
\left\langle\tau_{s}^{k}\right\rangle^{(\beta)}=\left(\frac{\beta}{2}\right)^{k} \frac{\left(\frac{\beta N}{2}-k\right) !}{\left(\frac{\beta N}{2}\right) !} \text { for } k<\frac{\beta N}{2}+1
$$

That is, the distribution for the partial times can be also generalized to any $\beta$ and $N$ by replacing $N$ by $\beta N / 2$ in the corresponding distribution of the $\beta=2$ case of Eq. (5). ${ }^{36-38}$ The quantitative difference given by the factor $K_{N}^{(\beta)}(k, 0, \ldots, 0)$ in Eq. (85), comes from the level repulsion for the proper delay times, as happens for their corresponding density. ${ }^{2}$ The only exception to this rule is, of course, the case $N=1$.

We can also notice that the factor $K_{N}^{(\beta)}(k, 0, \ldots, 0)$ has the following expression:

$$
K_{N}^{(\beta)}(k, 0, \ldots, 0)=\frac{k ! N^{k-1} N !}{(N+k-1) !} \quad \text { for } \quad k=1,2,3,
$$

independent of $\beta$.

From Eqs. (85) and (87), we obtain the following interesting averages:

$$
\left\langle q_{1}\right\rangle^{(\beta)}=\frac{1}{N}, \quad\left\langle q_{1}^{2}\right\rangle^{(\beta)}=\frac{\beta}{2} \frac{2}{\left(\frac{\beta N}{2}-1\right)(N+1)},
$$

and

$$
\left\langle q_{1}^{3}\right\rangle^{(\beta)}=\left(\frac{\beta}{2}\right)^{2} \frac{3 ! N}{\left(\frac{\beta N}{2}-1\right)\left(\frac{\beta N}{2}-2\right)(N+1)(N+2)}
$$

which agrees with those of Ref. 44 for $\beta=2$ in the semiclassical limit, and of Ref. 45 for any $\beta$ and $N$. Both results in Eq. (88) were reported in Refs. 24 and 28 for $\beta=1$ and 2. Another case that can be easily obtained from Eqs. (82) and (84), which is of particular interest, is

$$
\left\langle q_{1} q_{2}\right\rangle^{(\beta)}=\frac{1}{N(N+1)},
$$

which was reported in Ref. 29 for $\beta=2$.

Since the Wigner time delay is $\tau_{W}=\tau_{H} \sum_{i}^{N} q_{i} / N$, its mean and variance can be calculated from Eqs. (88) and (90) for any $\beta$ and $N$; the results are

$$
\tau_{W}=\frac{\tau_{H}}{N}, \quad \frac{\left\langle\tau_{W}^{2}\right\rangle-\left\langle\tau_{W}\right\rangle^{2}}{\left\langle\tau_{W}\right\rangle^{2}}=\frac{2}{\left(\frac{\beta N}{2}-1\right)(N+1)},
$$

which for $\beta=2$ reduces to those of Refs. 36 and 37.

From Eqs. (57), (62), (63), the second equation in (71), and Eq. (77), is feasible to find that

$$
K_{N}^{(\beta)}(2,2,0, \ldots, 0)=\frac{2 N(2 N+1)}{(N+2)(N+3)} .
$$


TABLE I. Summary of results for other moments for $\beta=1,2,4$, some of which were obtained numerically.

\begin{tabular}{|c|c|c|c|c|c|c|c|c|c|c|c|c|}
\hline \multirow[b]{2}{*}{$N$} & \multicolumn{4}{|c|}{$\beta=1$} & \multicolumn{4}{|c|}{$\beta=2$} & \multicolumn{4}{|c|}{$\beta=4$} \\
\hline & 2 & 3 & 4 & 5 & 2 & 3 & 4 & 5 & 2 & 3 & 4 & 5 \\
\hline$\left\langle q_{1}^{2} q_{2}\right\rangle$ & $\cdots$ & $\cdots$ & $\frac{4 \cdot 6 \cdot 1 !}{6 !}$ & & $\frac{2 \cdot 2 \cdot 0 !}{4 !}$ & $\frac{2 \cdot 3 \cdot 1 !}{5 !}$ & $\frac{2 \cdot 4 \cdot 2 !}{6 !}$ & $\frac{2 \cdot 5 \cdot 3 !}{7 !}$ & $\frac{4 \cdot 2 \cdot 1 !}{3 \cdot 4 !}$ & $\frac{4 \cdot 3 \cdot 2 !}{5 \cdot 5 !}$ & $\frac{4 \cdot 4 \cdot 3 !}{7 \cdot 6 !}$ & $\frac{4 \cdot 5 \cdot 4 !}{9 \cdot 7 !}$ \\
\hline$\left\langle q_{1}^{3} q_{2}\right\rangle$ & $\ldots$ & $\ldots$ & $\cdots$ & & $\ldots$ & $\frac{2 \cdot 31 \cdot 0 !}{6 !}$ & $\frac{2 \cdot 53 \cdot 1 !}{7 !}$ & $\frac{2 \cdot 81 \cdot 2 !}{8 !}$ & $\frac{4 \cdot 2 \cdot 7 \cdot 0 !}{3 \cdot 5 !}$ & $\frac{4 \cdot 3 \cdot 10 \cdot 1 !}{5 \cdot 6 !}$ & $\frac{4 \cdot 4 \cdot 13 \cdot 2 !}{7 \cdot 7 !}$ & $\frac{4 \cdot 5 \cdot 16 \cdot 3 !}{9 \cdot 8 !}$ \\
\hline$\left\langle q_{1}^{3} q_{2}^{2}\right\rangle$ & $\cdots$ & $\cdots$ & $\cdots$ & & $\ldots$ & $\frac{6 \cdot 3 \cdot 7 \cdot 0 !}{7 !}$ & $\frac{6 \cdot 4 \cdot 9 \cdot 1 !}{8 !}$ & $\frac{6 \cdot 5 \cdot 11 \cdot 2 !}{9 !}$ & $\frac{6 \cdot 4 \cdot 4 \cdot 0 !}{3 \cdot 6 !}$ & $\frac{6 \cdot 4 \cdot 9 \cdot 1 !}{5 \cdot 7 !}$ & $\frac{6 \cdot 4 \cdot 16 \cdot 2 !}{7 \cdot 8 !}$ & $\frac{6 \cdot 4 \cdot 25 \cdot 3 !}{9 \cdot 9 !}$ \\
\hline$\left\langle q_{1}^{2} q_{2} q_{3}\right\rangle$ & $\ldots$ & $\frac{2 \cdot 0 !}{5 !}$ & $\frac{2 \cdot 12 \cdot 1 !}{7 !}$ & & $\ldots$ & $\frac{2 \cdot 3 \cdot 1 !}{6 !}$ & $\frac{2 \cdot 4 \cdot 2 !}{7 !}$ & $\frac{2 \cdot 5 \cdot 3 !}{8 !}$ & $\ldots$ & $\frac{4 \cdot 3 \cdot 2 !}{5 \cdot 6 !}$ & $\frac{4 \cdot 4 \cdot 3 !}{7 \cdot 7 !}$ & $\frac{4.5 \cdot 4 !}{9 \cdot 8 !}$ \\
\hline$\left\langle q_{1}^{2} q_{2}^{2} q_{3}\right\rangle$ & $\cdots$ & $\cdots$ & $\frac{2 \cdot 36 \cdot 1 !}{8 !}$ & $\cdots$ & $\frac{2 \cdot 7 \cdot 1 !}{7 !}$ & $\frac{2 \cdot 9 \cdot 2 !}{8 !}$ & $\frac{2 \cdot 11 \cdot 3 !}{9 !}$ & $\ldots$ & $\frac{8 \cdot 3 \cdot 2 !}{5 \cdot 7 !}$ & $\frac{8.4 \cdot 3 !}{7 \cdot 8 !}$ & $\frac{8.5 \cdot 4 !}{9 \cdot 9 !}$ & \\
\hline
\end{tabular}

Although we do not have explicit results for $\beta=1$, we have verified this expression numerically. This result allows to obtain

$$
\left\langle q_{1}^{2} q_{2}^{2}\right\rangle^{(\beta)}=\left(\frac{\beta}{2}\right)^{4} \frac{\left(\frac{\beta N}{2}-2\right) !}{\left(\frac{\beta N}{2}\right) !} \frac{\left[\frac{\beta(N+1)}{2}-2\right] !}{\left[\frac{\beta(N+1)}{2}\right] !} K_{N}^{(\beta)}(2,2,0, \ldots, 0) .
$$

In Table I, we summarize some moments expressed in Eqs. (58), (65), (66), (80), and others that were obtained numerically. They can easily be generalized to

$$
\left\langle q_{1}^{2} q_{2}\right\rangle^{(\beta)}=\left(\frac{\beta}{2}\right)^{3} \frac{\left(\frac{\beta N}{2}-2\right) !}{\left(\frac{\beta N}{2}\right) !} \frac{\left[\frac{\beta(N+1)}{2}-1\right] !}{\left[\frac{\beta(N+1)}{2}\right] !} K_{N}^{(\beta)}(2,1,0,0, \ldots, 0)
$$

with

$$
\begin{gathered}
K_{N}^{(\beta)}(2,1,0,0, \ldots, 0)=\frac{2 N}{N+2} \\
\left\langle q_{1}^{3} q_{2}\right\rangle^{(\beta)}=\left(\frac{\beta}{2}\right)^{4} \frac{\left(\frac{\beta N}{2}-3\right) !}{\left(\frac{\beta N}{2}\right) !} \frac{\left[\frac{\beta(N+1)}{2}-1\right] !}{\left[\frac{\beta(N+1)}{2}\right] !} K_{N}^{(\beta)}(3,1,0,0, \ldots, 0),
\end{gathered}
$$

where

$$
\begin{gathered}
K_{N}^{(\beta)}(3,1,0,0, \ldots, 0)=\frac{2\left[3 N^{2}+N+(2-\beta / 2)\right]}{(N+3)(N+2)} \\
\left\langle q_{1}^{3} q_{2}^{2}\right\rangle^{(\beta)}=\left(\frac{\beta}{2}\right)^{5} \frac{\left(\frac{\beta N}{2}-3\right) !}{\left(\frac{\beta N}{2}\right) !} \frac{\left[\frac{\beta(N+1)}{2}-2\right] !}{\left[\frac{\beta(N+1)}{2}\right] !} K_{N}^{(\beta)}(3,2,0,0, \ldots, 0),
\end{gathered}
$$

with

$$
\begin{gathered}
K_{N}^{(\beta)}(3,2,0,0, \ldots, 0)=\frac{6 N^{2}(2 N+1)}{(N+4)(N+3)(N+2)} \\
\left\langle q_{1}^{2} q_{2} q_{3}\right\rangle^{(\beta)}=\left(\frac{\beta}{2}\right)^{4} \frac{\left(\frac{\beta N}{2}-2\right) !}{\left(\frac{\beta N}{2}\right) !} \frac{\left[\frac{\beta(N+1)}{2}-1\right] !}{\left[\frac{\beta(N+1)}{2}\right] !} \frac{\left[\frac{\beta(N+2)}{2}-1\right] !}{\left[\frac{\beta(N+2)}{2}\right] !} K_{N}^{(\beta)}(2,1,1,0, \ldots, 0),
\end{gathered}
$$

where

$$
K_{N}^{(\beta)}(2,1,1,0, \ldots, 0)=\frac{2 N}{N+3}
$$


and

$$
\left\langle q_{1}^{2} q_{2}^{2} q_{3}\right\rangle^{(\beta)}=\left(\frac{\beta}{2}\right)^{5} \frac{\left(\frac{\beta N}{2}-2\right) !}{\left(\frac{\beta N}{2}\right) !} \frac{\left[\frac{\beta(N+1)}{2}-2\right] !}{\left[\frac{\beta(N+1)}{2}\right] !} \frac{\left[\frac{\beta(N+2)}{2}-1\right] !}{\left[\frac{\beta(N+2)}{2}\right] !} K_{N}^{(\beta)}(2,2,1,0, \ldots, 0)
$$

with

$$
K_{N}^{(\beta)}(2,2,1,0, \ldots, 0)=\frac{2 N(2 N+1)}{(N+4)(N+3)} .
$$

The results of Eqs. (93), (96), and (100) were reported in Ref. 29 for $\beta=2$.

\section{CONCLUSIONS}

We have calculated generalized joint moments of proper delay times for an arbitrary number of channels $N$ and any symmetry $\beta=1,2$, and 4 , which are needed to quantify transport properties, or their fluctuations, through ballistic open systems. This was done by reducing the calculation of the negative moments of the generalized Laguerre distribution to simpler formulas, which are easier to manage analytically. As an important result, we show that the $k$ th moment of a proper delay time differs from that of the partial delay time, where the difference comes from the level repulsion of the proper delay times. From our results, also, we were able to generalize the distribution of the partial times to any symmetry. Our general expressions reproduce the existing results for particular cases and those obtained for individual proper and partial delay times. Also, we obtained the mean and variance of the Wigner time delay for arbitraries $N$ and $\beta$, which reproduces the known results for $\beta=2$. Although we regarded perfect coupling of the system to the open channels, we hope that our results encourage further calculations to include an imperfect coupling, as was done for partial delay times.

\section{ACKNOWLEDGMENTS}

M. Martínez-Mares and J. C. García are grateful to the Sistema Nacional de Investigadores, Mexico. M. Martínez-Mares is also grateful to M. A. Torres-Segura for her encouragement. A. M. Martínez-Argüello thanks CONACyT, Mexico for financial support.

\section{APPENDIX: PROPERTIES ASSOCIATED TO $\boldsymbol{F}_{\boldsymbol{\theta}_{\boldsymbol{n}}}$}

There are some relations between the Gamma, Poisson, and Negative Binomial probability distributions that are summarized in the following propositions:

Proposition 1. For $b, b_{1}, b_{2}>0, k, m, n \in \mathbb{Z}_{+}$, and $x \geq 0$,

$$
\begin{aligned}
& F_{n, b}(x)=\int_{0}^{x} f_{n, b}(t) \mathrm{d} t=\sum_{k=n}^{\infty} P_{\text {Poisson }}(b x, k), \\
& \int_{0}^{x} f_{m, b_{2}}(t) P_{\text {Poisson }}\left(b_{1} t, k\right) \mathrm{d} t=N B_{m, p}(k) F_{m+k, b_{1}+b_{2}}(x),
\end{aligned}
$$

where $p=b_{2} /\left(b_{1}+b_{2}\right)$ and $P_{\text {Poisson }}(\lambda, k)$ is the Poisson distribution with parameter $\lambda$ given by

$$
P_{\text {Poisson }}(\lambda, k)=\frac{\lambda^{k}}{k !} \mathrm{e}^{-\lambda}, \quad \text { with } \quad k=0,1, \ldots,
$$

and $N B_{m, p}(k)$ is the Negative Binomial distribution ${ }^{52}$ with parameters $m \in \mathbb{N}$ and $p \in[0,1]$ :

$$
N B_{m, p}(k)=\left(\begin{array}{c}
m-1+k \\
m-1
\end{array}\right)(1-p)^{k} p^{m}, \quad \text { with } \quad k=0,1, \ldots
$$


Proof. The proof of (A1) is based on the following identity, which is proved by integrating by parts and induction on $n$ (see Chap. 4, exercise 26, p. 200 of Ref. 51),

$$
\frac{1}{n !} \int_{x}^{\infty} t^{n} \mathrm{e}^{-t} \mathrm{~d} t=\sum_{k=0}^{n} \mathrm{e}^{-x} \frac{x^{k}}{k !}
$$

Therefore, with the change of variables $u=b t$ we have that

$$
F_{n, b}(x)=1-\frac{1}{(n-1) !} \int_{b x}^{\infty} u^{n-1} \mathrm{e}^{-u} \mathrm{~d} u=\sum_{k=n}^{\infty} \frac{(b x)^{k}}{k !} \mathrm{e}^{-b x}=\sum_{k=n}^{\infty} P_{\text {Poisson }}(b x, k) .
$$

To prove (A2), let $p=b_{2} /\left(b_{1}+b_{2}\right)$. Then,

$$
\int_{0}^{x} f_{m, b_{2}}(t) P_{\text {Poisson }}\left(b_{1} t, k\right) \mathrm{d} t=\frac{b_{2}^{m} b_{1}^{k}}{(m-1) ! k !} \int_{0}^{x} t^{m+k-1} \mathrm{e}^{-\left(b_{1}+b_{2}\right) t} \mathrm{~d} t=N B_{m, p}(k) F_{m+k, b_{1}+b_{2}}(x) .
$$

Let

$$
F_{\theta_{n}}^{(n)}(x):=\int_{\Delta_{n}(x)} \prod_{i=1}^{n} f_{a_{i}, b_{i}}\left(t_{i}\right) \mathrm{d} t_{i}
$$

where $\Delta_{n}(x)=\left\{\left(t_{1}, \ldots, t_{n}\right): 0 \leq t_{1} \leq t_{2} \leq \cdots \leq t_{n} \leq x\right\}$. By the Cavallieri Principle, the functions $F_{\theta_{n}}^{(n)}(x)$ satisfy the recurrence relation

$$
F_{\theta_{n}}^{(n)}(y)=\int_{0}^{y} f_{a_{n}, b_{n}}(x) F_{\theta_{n-1}}^{(n-1)}(x) \mathrm{d} x .
$$

Also, for this function we have the following properties:

Proposition 2. If $n \geq 2$, all the $a_{i} \in \mathbb{N}, g_{k}:=\sum_{i=1}^{k} b_{i}$, and $p_{k}:=b_{k} / g_{k}$, then

$$
\begin{aligned}
F_{\theta_{1}}^{(1)}(y) & =\sum_{k=a_{1}}^{\infty} P_{\text {Poisson }}\left(b_{1} y, k\right), \quad F_{\theta_{1}}=1, \\
F_{\theta_{n}}^{(n)}(x) & =\sum_{\mathbf{A}^{(n-1)}} \prod_{j=2}^{n} N B_{a_{j}, p_{j}}\left(L_{j-1}\right) F_{a_{n}+L_{n-1}, g_{n}}(x), \\
F_{\theta_{n}} & =\sum_{\mathbf{A}^{(n-1)}} \prod_{j=2}^{n} N B_{a_{j}, p_{j}}\left(L_{j-1}\right)
\end{aligned}
$$

where $L:=\sum_{i=1}^{n} \ell_{i}$, for $\ell_{i} \in \mathbb{N}, \sum_{\mathbf{A}^{(n-1)}}$ means summation over all $\ell_{i} \in \mathbf{A}^{(n-1)}$, with

$$
\mathbf{A}^{(n)}:=\left\{\ell_{1}, \ldots, \ell_{n}: \ell_{i} \geq a_{i}, \ell_{i} \in \mathbb{N}, i=1, \ldots, n\right\} .
$$

Proof. The first formula in (A10), is exactly (A1) of Proposition 1. The second is clear since $F_{\theta_{1}}^{(1)}$ is a probability distribution function. To prove (A11), we proceed by induction on $n$. For $n=2$, (A11) is a direct consequence of Proposition 1 , since $a_{1} \in \mathbb{N}$, and the Monotone Convergence Theorem to interchange the series and the integral. As inductive hypothesis, let us assume that (A11) holds for $n-1$, with some $n \geq 3$, and try to prove it for $n$. With this hypothesis, we write Eq. (A9) as

$$
\begin{aligned}
F_{\theta_{n}}^{(n)}(y) & =\sum_{\mathbf{A}^{(n-2)}} \prod_{j=2}^{n-1} N B_{a_{j}, p_{j}}\left(L_{j-1}\right) \int_{0}^{y} f_{a_{n}, b_{n}}(x) F_{a_{n-1}+L_{n-2}, g_{n-1}}(x) \mathrm{d} x \\
& =\sum_{\mathbf{A}^{(n-2)}} \prod_{j=2}^{n-1} N B_{a_{j}, p_{j}}\left(L_{j-1}\right) \sum_{k=a_{n-1}+L_{n-2}}^{\infty} N B_{a_{n}, p_{n}}(k) F_{a_{n}+k, g_{n}}(y),
\end{aligned}
$$


where at the last equality we first used (A1) and then (A2) with $m=a_{n}, k=L_{n-2}, p=p_{n}$, taking into account that $p_{n}=b_{n} /\left(b_{n}+g_{n-1}\right)$. If we make the change of variables $\ell_{n-1}=k-L_{n-2}$ we write the last equation as

$$
\begin{aligned}
F_{\theta_{n}}^{(n)}(y) & =\sum_{\mathbf{A}^{(n-2)}} \prod_{j=2}^{n-1} N B_{a_{j}, p_{j}}\left(L_{j-1}\right) \sum_{\ell_{n-1}=a_{n-1}}^{\infty} N B_{a_{n}, p_{n}}\left(\ell_{n-1}+L_{n-2}\right) F_{a_{n}+\ell_{n-1}+L_{n-2}, g_{n}}(y) \\
& =\sum_{\mathbf{A}^{(n-1)}} \prod_{j=2}^{n} N B_{j, p_{j}}\left(L_{j-1}\right) F_{a_{n}+L_{n-1}, g_{n}}(y) .
\end{aligned}
$$

At the last step, we used the sets $\mathbf{A}^{(n)}$ which are Cartesian products [see (A13)] in fact,

$$
\mathbf{A}^{(n-1)}=\mathbf{A}^{(n-2)} \times\left\{a_{n-1}, 1+a_{n-1}, 2+a_{n-1}, \ldots\right\} .
$$

This finishes the proof of (A11).

The proof of (A12) is based on the Monotone Convergence Theorem. Indeed,

$$
F_{\theta_{n}}=\lim _{x \rightarrow \infty} F_{\theta_{n}}^{(n)}(x)=\sum_{\mathbf{A}^{(n-1)}} \prod_{j=2}^{n} N B_{a_{j}, p_{j}}\left(L_{j-1}\right) \lim _{x \rightarrow \infty} F_{a_{n}+L_{n-1}, g_{n}}(x)=\sum_{\mathbf{A}^{(n-1)}} \prod_{j=2}^{n} N B_{a_{j}, p_{j}}\left(L_{j-1}\right)
$$

Remark 3. It is not necessary, for Proposition 2 to hold, that the $b_{i}$ 's belong to $\mathbb{N}$, just that they are positive.

${ }^{1}$ P. W. Brouwer, K. M. Frahm, and C. W. J. Beenakker, Phys. Rev. Lett. 78, 4737 (1997).

${ }^{2}$ P. W. Brouwer, K. M. Frahm, and C. W. J. Beenakker, Waves Random Media 9, 91 (1999).

${ }^{3}$ F. T. Smith, Phys. Rev. 118, 349 (1960).

${ }^{4}$ E. P. Wigner, Phys. Rev. 98, 145 (1955).

${ }^{5}$ P. A. Mello and N. Kumar, Quantum Transport in Mesoscopic Systems: Complexity and Statistical Fluctuations (Oxford University Press, New York, 2005).

${ }^{6}$ J. Feist, O. Zatsarinny, S. Nagele, R. Pazourek, J. Burgdörfer, X. Guan, K. Bartschat, and B. I. Schneider, Phys. Rev. A 89, 033417 (2014).

${ }^{7}$ I. A. Ivanov and A. S. Kheifets, Phys. Rev. A 89, 043405 (2014).

${ }^{8}$ P. C. Deshmukh, A. Mandal, S. Saha, A. S. Kheifets, V. K. Dolmatov, and S. T. Manson, Phys. Rev. A 89, 053424 (2014).

${ }^{9}$ A. Chacon, M. Lein, and C. Ruiz, Phys. Rev. A 89, 053427 (2014).

${ }^{10}$ M. Schultze, M. Fieß, N. Karpowicz, J. Gagnon, M. Korbman, M. Hofstetter, S. Neppl, A. L. Cavalieri, Y. Komninos, Th. Mercouris, C. A. Nicolaides, R. Pazourek, S. Nagele, J. Feist, J. Burgdörfer, A. M. Azzeer, R. Ernstorfer, R. Kienberger, U. Kleineberg, E. Goulielmakis, F. Krausz, and V. S. Yakovlev, Science 328, 1658 (2010).

${ }^{11}$ P. A. Mello, in Mesoscopic Quantum Physics, edited by E. Akkermans, G. Montambaux, J.-L. Pichard, and J. Zinn-Justin (Elsevier, Amsterdam, 1995).

${ }^{12}$ C. W. J. Beenakker, Rev. Mod. Phys. 69, 731 (1997).

${ }^{13}$ Y. Alhassid, Rev. Mod. Phys. 72, 895 (2000).

${ }^{14}$ Y. V. Fyodorov, D. V. Savin, and H.-J. Sommers, J. Phys. A: Math. Gen. 38, 10731 (2005).

${ }^{15}$ B. D. Simons and B. L. Altshuler, Phys. Rev. B 48, 5422 (1993).

${ }^{16}$ Y. V. Fyodorov, Phys. Rev. Lett. 73, 2688 (1994).

${ }^{17}$ N. Taniguchi, A. Hashimoto, B. D. Simons, and B. L. Altshuler, Europhys. Lett. 27, 335 (1994).

${ }^{18}$ Y. V. Fyodorov and A. D. Mirlin, Phys. Rev. B 51, 13403 (1995).

${ }^{19}$ C. M. Marcus, A. J. Rimberg, R. M. Westervelt, P. F. Hopkins, and A. C. Gossard, Phys. Rev. Lett. 69, 506 (1992).

${ }^{20}$ M. W. Keller, A. Mittal, J. W. Sleight, R. G. Wheeler, D. E. Prober, R. N. Sacks, and H. Shtrikmann, Phys. Rev. B 53, R1693 (1996).

${ }^{21}$ I. H. Chan, R. M. Clarke, C. M. Marcus, K. Campman, and A. C. Gossard, Phys. Rev. Lett. 74, 3876 (1995).

22 A. G. Huibers, S. R. Patel, C. M. Marcus, P. W. Brouwer, C. I. Duruöz, and J. S. Harris, Jr., Phys. Rev. Lett. 81, 1917 (1998).

${ }^{23}$ P. W. Brouwer, S. A. van Langen, K. M. Frahm, M. Büttiker, and C. W. J. Beenakker, Phys. Rev. Lett. 79, 913 (1997).

${ }^{24}$ M. Martínez-Mares and E. Castaño, Phys. Rev. E 71, 036201 (2005).

${ }^{25}$ M. Switkes, C. M. Marcus, K. Campman, and A. C. Gossard, Science 283, 1905 (1999).

${ }^{26}$ P. W. Brouwer, Phys. Rev. B 58, R10135 (1998).

${ }^{27}$ H. Schanze, H.-J. Stöckmann, M. Martínez-Mares, and C. H. Lewenkopf, Phys. Rev. E 71, 016223 (2005).

${ }^{28}$ M. Martínez-Mares, Phys. Rev. E 72, 036202 (2005).

${ }^{29}$ M. Martínez-Mares, C. H. Lewenkopf, and E. R. Mucciolo, Phys. Rev. B 69, 085301 (2004).

${ }^{30}$ V. A. Gopar, P. A. Mello, and M. Büttiker, Phys. Rev. Lett. 77, 3005 (1996).

${ }^{31}$ Y. V. Fyodorov, D. V. Savin, and H.-J. Sommers, Phys. Rev. E 55, R4857 (1997). 
${ }^{32}$ A. Ossipov, T. Kottos, and T. Geisel, Europhys. Lett. 62, 719 (2003).

${ }^{33}$ A. Ossipov and Y. V. Fyodorov, Phys. Rev. B 71, 125133 (2005).

${ }^{34}$ T. Kottos and M. Weiss, Phys. Rev. Lett. 89, 056401 (2002).

35 J. A. Méndez-Bermúdez and T. Kottos, Phys. Rev. E 72, 064108 (2005).

${ }^{36}$ Y. V. Fyodorov and H.-J. Sommers, Phys. Rev. Lett. 76, 4709 (1996).

${ }^{37}$ Y. V. Fyodorov and H.-J. Sommers, J. Math. Phys. 38, 1918 (1997).

${ }^{38}$ P. Šeba, K. Życzkowski, and J. Zakrzewski, Phys. Rev. E 54, 2438 (1996).

${ }^{39}$ C. Texier and A. Comtet, Phys. Rev. Lett. 82, 4220 (1999).

${ }^{40}$ A. Ossipov, T. Kottos, and T. Geisel, Phys. Rev. B 61, 11411 (2000).

${ }^{41}$ D. V. Savin, Y. V. Fyodorov, and H.-J. Sommers, Phys. Rev. E 63, 035202(R) (2001).

${ }^{42}$ H.-J. Sommers, D. V. Savin, and V. V. Sokolov, Phys. Rev. Lett. 87, 094101 (2001).

${ }^{43}$ M. Marciani, P. W. Brouwer, and C. W. J. Beenakker, Phys. Rev. B 90, 045403 (2014).

${ }^{44}$ G. Berkolaiko and J. Kuipers, J. Phys. A: Math. Theor. 43, 035101 (2010).

${ }^{45}$ F. Mezzadri and N. J. Simm, J. Math. Phys. 52, 103511 (2011).

${ }^{46}$ F. Mezzadri and N. J. Simm, J. Math. Phys. 53, 053504 (2012).

${ }^{47}$ F. J. Dyson, J. Math. Phys. 3, 140 (1962).

${ }^{48}$ I. S. Gradshteyn and I. M. Ryzhik, Table of Integrals, Seriesm and Products (Academic Press, Inc., Orlando, 1980), p. 1111.

${ }^{49}$ G. Birkhoff and S. Mac Lane, A Survey of Modern Algebra (AKP Classics, 1998).

${ }^{50}$ I. N. Herstein, Topics in Algebra, 2nd ed. (John Wiley \& Sons, New York, 1975).

${ }^{51}$ S. Ross, A First Course in Probability, 7th ed. (Pearson Prentice Hall, New Jersey, 2006).

${ }^{52}$ Handbook of Mathematical Functions, edited by M. Abramowitz and I. A. Stegun (Dover Publications, Inc., New York, 1972).

${ }^{53}$ The negative binomial distribution can be used only when $N$ is an even number. 
EPJ manuscript No.

(will be inserted by the editor)

\title{
Delay times in chaotic quantum systems
}

\author{
A. M. Martínez-Argüello1,a , A. A. Fernández-Marín ${ }^{1,2}$, and M. Martínez-Mares ${ }^{1, b}$ \\ 1 Departamento de Física, Universidad Autónoma Metropolitana-Iztapalapa, Apartado Postal \\ 55-534, 09340 Ciudad de México, Mexico \\ 2 Instituto de Ciencias Físicas, Universidad Nacional Autónoma de México, Apartado Postal \\ 48-3, 62210 Cuernavaca Mor., Mexico
}

\begin{abstract}
Based on recent results of the joint moments of proper delay times of open chaotic systems with ideal coupling, a new insight to obtain the partial delay times distribution, for an arbitrary number of channels and symmetry, is given. This distribution is completely verified for all symmetry classes by means of random matrix theory simulations of ballistic chaotic cavities. In addition, the normalization constant of the Laguerre ensemble is obtained.
\end{abstract}

\section{Introduction}

The delay experienced by a quantum particle due to interactions with a scattering region has been the subject of intense investigation for more than thirty years in several areas of physics that include nuclear and condensed matter physics [1-7]. The interest in this subject has resurged due to the recent appearance of theoretical investigations in chaotic systems [8-14] and atomic physics [15-18]; the later motivated by experiments of interaction of light with matter during a mean time with attosecond precision [19].

The delay time first introduced by Wigner for the one channel case [1] and its multichannel generalization by Smith [2], in the so-called Wigner-Smith time delay matrix, is written in terms of the scattering matrix $S$ and its derivative with respect to the energy $\varepsilon$. In units of the Heisenberg time $\tau_{\mathrm{H}}$, it is given by

$$
Q_{\mathrm{W}}=-\mathrm{i} \frac{\hbar}{\tau_{\mathrm{H}}} S^{-1} \frac{\partial S}{\partial \varepsilon} .
$$

The eigenvalues of $Q_{\mathrm{w}}$ represent the delay time on each channel and the Wigner time delay is the average of these proper delay times. In the context of mesoscopic systems the electrochemical capacitance of a mesoscopic capacitor is described by the Wigner time delay [20-22]. Some other transport observables that depend on the proper delay times are the thermopower [23], the derivative of the conductance with respect to the Fermi energy [24], the DC pumped current at zero bias [25], among others (see for instance Ref. [26] and references there in). For ballistic systems with chaotic classical dynamics, these physical observables fluctuate with respect to small

\footnotetext{
a e-mail: angelma@xanum.uam.mx

b e-mail: moi@xanum.uam.mx
} 
variations of external parameters, like an applied magnetic field, the Fermi energy or the system shape [26-28]. The proper delay times are of interest in the characterization of those fluctuations, which only depend on the symmetry present in the problem. The distribution of the proper delay times is given in terms of the joint distribution of their reciprocals, known as the Laguerre ensemble $[26,28]$. An interesting feature of this ensemble is the presence of repulsion between the proper times, as occurs in the spectral statistics.

Alternatively, the partial delay times defined as the energy derivative of the phase shifts are also useful in the characterization of chaotic scattering [27]. Although the partial times are correlated, this correlation is of different nature than that of between the proper delay times; they do not show the level repulsion [29]. In the one channel situation the proper and partial delay times are identical to the Wigner time delay whose distribution is known for all symmetry classes $[27,30,31]: \beta=1$ (4) in the presence of time reversal and presence (absence) of spin-rotation symmetry and $\beta=2$ in the absence of time reversal symmetry. For the general case of arbitrary number of channels the distribution of the partial times is known for any $\beta$, except by a normalization constant [29]. With a suitable normalization this result encompasses a previous one for $\beta=2[27,32]$. Numerical simulations for $\beta=1$ and 2 have been given in Ref. [33], but only the $\beta=2$ case was succesfully compared with the appropriate theoretical result. The $\beta=4$ symmetry is seldom discussed.

In the present paper, an alternative approach to verify the general expression for the properly normalized probability distribution of the partial delay times is obtained. This was done by extracting the essence that comes from the level repulsion in the joint distribution of proper delay times that transcends to the $k$ th moment of a proper delay time [34]. This procedure leads to the distribution of partial times in the equivalent channels situation, that we test by means of random matrix theory simulations, for all symmetry classes and several number of channels. In addition, this method also allows us to obtain the normalization constant of the Laguerre distribution.

In the next section we establish the theoretical framework of the proper and partial delay times; we review the known results for the $k$ th moment of the proper delay times, from which we obtain the general expression of the probability distribution of the partial times, for all symmetry classes and any number of channels. Also, it is in this section where we present our findings of the normalization constant of the Laguerre distribution. In Sect. 3 we compare this general distribution with the numerical predictions from random matrix theory. We conclude in Sect. 4.

\section{Distributions of proper and partial delay times}

\subsection{Scattering approach}

Single-electron scattering by a ballistic cavity attached ideally to two leads which support $N_{1}$ and $N_{2}$ propagating modes (channels), respectively, can be described by an $N \times N$ scattering matrix $S$, where $N=N_{1}+N_{2}$. When the dynamics of the cavity is classically chaotic, the scattering matrix belongs to one of the three circular ensembles from random matrix theory (RMT) $[35,36]$. The Circular Unitary Ensemble (CUE) is obtained when flux conservation is the only restriction in the problem, such that $S^{\dagger} S=1_{N}$, where $1_{N}$ denotes the unit matrix of dimension $N$. In the Dyson scheme this case is labeled by $\beta=2$. Additionally, in the presence of time reversal invariance (TRI) and integral spin or TRI, half-integral spin, and

rotation symmetry, $S$ is a symmetric matrix, $S=S^{T}$ (the upper script $T$ means transpose). This case is denoted by $\beta=1$ and the corresponding ensemble is the 


\begin{tabular}{c|ccccc}
$N$ & 1 & 2 & 3 & 4 & 5 \\
\hline$C_{N}^{(1)}$ & $\frac{(1 / 2)^{3 / 2}}{(1 / 2) !}$ & $\frac{1}{48}$ & $\frac{(1 / 2)^{3 / 2}}{(1 / 2) ! \cdot 180 \cdot 48}$ & $\frac{1}{53760 \cdot 180 \cdot 48}$ & $\cdots$ \\
\hline$C_{N}^{(2)}$ & $\frac{1}{1 !(1 !)}$ & $\frac{1}{2 !(3 ! \cdot 2 ! \cdot 1 !)}$ & $\frac{1}{3 !(5 ! \cdot 4 ! \cdot 3 ! \cdot 2 ! \cdot 1 !)}$ & $\frac{1}{4 !(7 ! \cdot 6 ! \cdot 5 ! \cdot 4 ! \cdot 3 ! \cdot 2 ! \cdot 1 !)}$ & $\frac{1}{5 !(9 ! \cdots 3 ! \cdot 2 ! \cdot 1 !)}$ \\
\hline$C_{N}^{(4)}$ & $\frac{2^{4}}{2 !(2 ! \cdot 0 !)}$ & $\frac{2^{16}}{4 !(6 ! \cdot 4 ! \cdot 2 ! \cdot 0 !)}$ & $\frac{2^{36}}{6 !(10 ! \cdot 8 ! \cdot 6 ! \cdot 4 ! \cdot 2 ! \cdot 0 !)}$ & $\frac{2^{64} \cdot(14 ! \cdot 12 ! \cdot 10 ! \cdot \cdot \cdot \cdot 6 ! \cdot 4 ! \cdot 2 ! \cdot 0 !)}{8 ! \cdot(0) !(18 ! \cdot \cdots 2 ! \cdot 0 !)}$ \\
\hline
\end{tabular}

Table 1. Summary of the results for the normalization constant $C_{N}^{(\beta)}$ of the Laguerre distribution for each symmetry class. For $\beta=1$ and 4 , the cases $N=1$ and 2 , and for $\beta=2$, the ones for $N=1,2,3$, and 4 , were reported in Ref. [34]. The remaining values were obtained numerically for the purpose of this paper.

Circular Orthogonal Ensemble (COE). In the presence of TRI, half-integral spin, and no rotation symmetry, $S$ is self-dual and the ensemble is the Circular Symplectic Ensemble (CSE), labeled by $\beta=4$.

In the diagonal form, the $S$ matrix can be written as

$$
S=U E U^{\dagger},
$$

where $U$ is an $N \times N$ unitary matrix, the matrix of eigenvectors, and $E$ is the diagonal matrix of eigenphases,

$$
E_{i j}=\mathrm{e}^{\mathrm{i} \theta_{i}} \delta_{i j}
$$

with $\delta_{i j}$ the Kronecker delta.

\subsection{Proper delay times}

A symmetrized form of the Wigner-Smith time delay matrix can be written in dimensionless units as $[26,28]$

$$
Q=-\mathrm{i} \frac{\hbar}{\tau_{\mathrm{H}}} S^{-1 / 2} \frac{\partial S}{\partial \varepsilon} S^{-1 / 2},
$$

where $\varepsilon$ is the energy and $\tau_{\mathrm{H}}$ is the Heisenberg time $\left(\tau_{\mathrm{H}}=2 \pi \hbar / \Delta\right.$, with $\Delta$ the mean level spacing). The matrix $Q$ is Hermitian for $\beta=2$, real symmetric for $\beta=1$, and quaternion self-dual for $\beta=4$. Its eigenvalues, $q_{i}$ 's $(i=1, \ldots, N)$ are the proper delay times measured in units of $\tau_{\mathrm{H}}$. The distribution of the $q_{i}$ 's is given by the Laguerre ensemble in terms of their reciprocals $x_{i}=1 / q_{i}[28]$, namely

$$
p_{\beta}\left(\left\{x_{i}\right\}\right)=C_{N}^{(\beta)} \prod_{a<b}^{N}\left|x_{b}-x_{a}\right|^{\beta} \prod_{c=1}^{N} x_{c}^{\beta N / 2} \mathrm{e}^{-\beta x_{c} / 2},
$$

where $C_{N}^{(\beta)}$ is a normalization constant. It is worth mentioning that the repulsion between the proper delay times is inherited from the level repulsion of the Hamiltonian eigenvalues. The normalization constant for the energy level distribution is well known [37], but the constant $C_{N}^{(\beta)}$ in Eq. (5) has not been given yet, although the Laguerre distribution has been widely used.

Here, we follow an inductive method to obtain a general expression for $C_{N}^{(\beta)}$. A summary of the results for this normalization constant previously reported in Ref. [34], as well as new others, are shown in Table 1 . We notice that the results for $\beta=2$ suggest a general dependence on $N$, namely

$$
C_{N}^{(2)}=\frac{1}{N !} \prod_{n=0}^{2 N-1} \frac{1}{n !}
$$


that gives an indication for the other two symmetry classes. For example, for $\beta=4$ the normalization constant can be written as

$$
C_{N}^{(4)}=\frac{2^{(2 N)^{2}}}{(2 N) !} \prod_{n=0}^{2 N-1} \frac{1}{(2 n) !} .
$$

The dependence on $N$ of the normalization constant for $\beta=1$ is more complicated than the corresponding ones for $\beta=2$ and 4 , it can be obtained in similar manner with the result

$$
C_{N}^{(1)}=\frac{\left[\left(\frac{1}{2}\right) !\right]^{N}}{2^{N(N+1 / 2)}\left(\frac{N}{2}\right) !} \prod_{n=0}^{2 N-1} \frac{1}{\left(\frac{n}{2}\right) !} .
$$

From these expressions it is straightforward to arrive at the general result for $C_{N}^{(\beta)}$; that is

$$
C_{N}^{(\beta)}=\frac{\left[\left(\frac{\beta}{2}\right)^{\beta(N-1 / 2)+1}\left(\frac{\beta}{2}\right) !\right]^{N}}{\left(\frac{\beta N}{2}\right) !} \prod_{n=0}^{2 N-1} \frac{1}{\left(\frac{\beta n}{2}\right) !} .
$$

What is very interesting of this result is the quite similarity with the normalization constant of the joint probability density of the eigenvalues of the Hamiltonian for the Gaussian ensembles [37].

In addition, let us note that the $k$ th moment of a proper delay time, valid for any symmetry and an arbitrary number of channels, given by [34]

$$
\left\langle q_{i}^{k}\right\rangle^{(\beta)}=\left(\frac{\beta}{2}\right)^{k} \frac{\left(\frac{\beta N}{2}-k\right) !}{\left(\frac{\beta N}{2}\right) !} K_{N}^{(\beta)}(k, 0, \ldots, 0),
$$

with $0 \leq k<1+\beta N / 2$, shows the underlying part that comes from the repulsion in Eq. (5) through the factor $K_{N}^{(\beta)}(k, 0, \ldots, 0)$.

\subsection{Partial delay times}

Partial delay times, defined as the energy derivative of the diagonal form of the scattering matrix as in Eq. (1), are given, in dimensionless units, by $[27,33]$

$$
\hat{\tau}=-\mathrm{i} \frac{\hbar}{\tau_{\mathrm{H}}} E^{-1} \frac{\partial E}{\partial \varepsilon}
$$

This is an $N \times N$ diagonal matrix whose elements are

$$
\tau_{s}=\frac{\hbar}{\tau_{\mathrm{H}}} \frac{\partial \theta_{s}}{\partial \varepsilon}
$$

Once the inherent part of the repulsion in the $k$ th moment of the proper times has been identified, it is straightforward to arrive at the expression of the $k$ th moment of the partial times since they do not show that repulsion; for equivalent channels it is $[34]$

$$
\left\langle\tau_{s}^{k}\right\rangle^{(\beta)}=\left(\frac{\beta}{2}\right)^{k} \frac{\left(\frac{\beta N}{2}-k\right) !}{\left(\frac{\beta N}{2}\right) !} .
$$



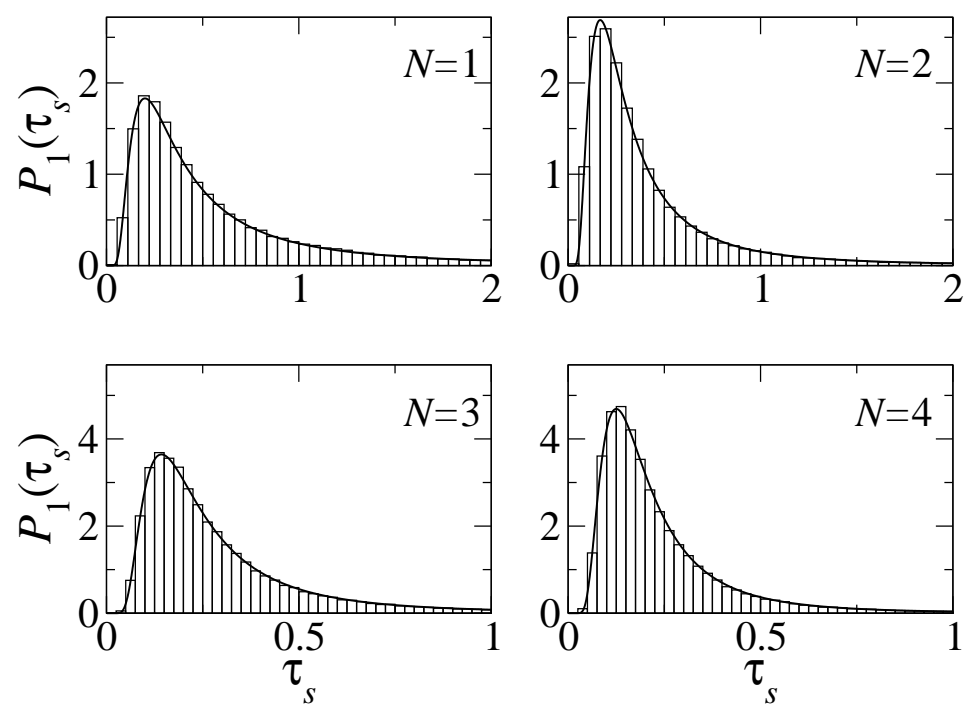

Fig. 1. Comparison between the numerical simulations (histograms) and theory (continuous lines), Eq. (14), for the distribution of $\tau_{s}$ (we take $s=1$ ) in the $\beta=1$ case.

This expression is in agreement with the results that can be obtained directly from the distribution for $N=1$ in Ref. [30]. Also, Eq. (13) includes the known result for $\beta=2$ and arbitrary $N[27,32]$ and is consistent with the distribution

$$
P_{\beta}\left(\tau_{s}\right)=\frac{2 / \beta}{\left(\frac{\beta N}{2}\right) !}\left(\frac{\beta}{2 \tau_{s}}\right)^{2+\beta N / 2} \mathrm{e}^{-\beta / 2 \tau_{s}} .
$$

Our expression (14) encompasses the existing results in the literature for $\beta=$ $2[27,30-33]$ and agrees with the distribution of partial times previously obtained in Ref. $[29]^{1}$.

In what follows we verify our findings with random matrix theory simulations.

\section{Numerical calculations}

The Hamiltonian approach, also known as the Heidelberg approach, is the best suited for the calculation of the energy derivative of the scattering matrix since it is written explicitly in terms of the energy, namely [37-39]

$$
S(\varepsilon)=1_{N}-2 \mathrm{i} \pi W^{\dagger} \frac{1}{\varepsilon 1_{M}-H+\mathrm{i} \pi W W^{\dagger}} W,
$$

where $H$ is an $M$-dimensional Hamiltonian matrix that describes the chaotic dynamics of the system, with $M$ resonant single-particle states, and $W$ is an $M \times N$ matrix, independent of the energy, which couples these resonant states to the $N$ propagating modes in the leads; $1_{n}$ stands for the unit matrix of dimension $n$. For ideal coupling of uncorrelated equivalent channels, $W_{\mu n}=\sqrt{M \Delta} / \pi(\mu=1, \ldots, M$ and $n=1, \ldots, N)$ for the matrix elements of $W$ [39].

\footnotetext{
${ }^{1}$ A misprint appears in the normalization constant in Eq. (11) of Ref. [29].
} 

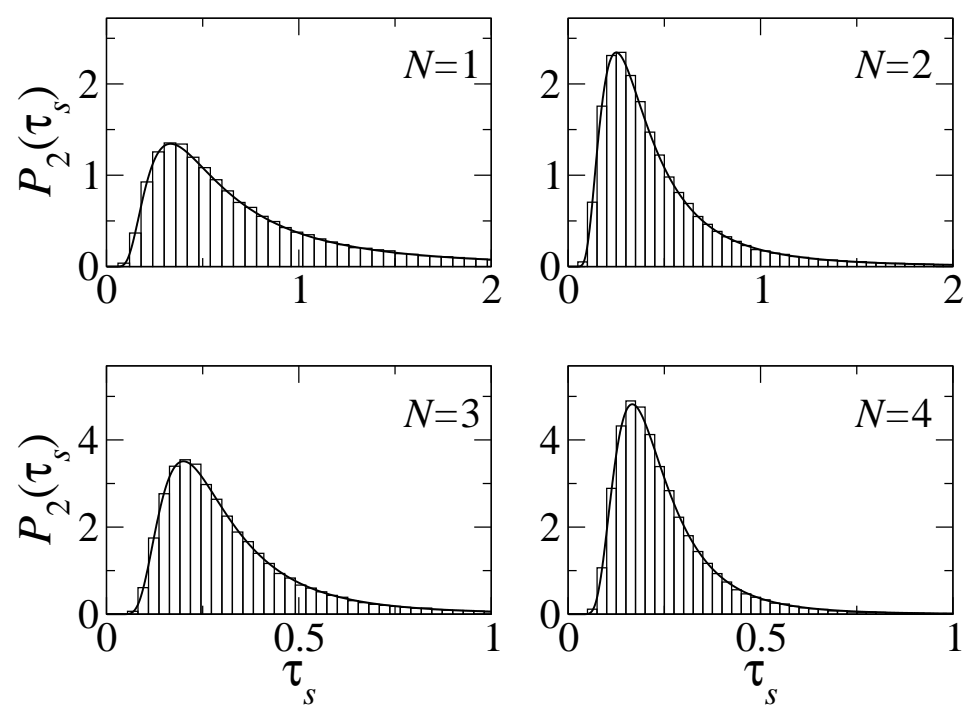

Fig. 2. The same as in Fig. 1 but for $\beta=2$.

For chaotic systems, $H$ is a random matrix chosen from one of the Gaussian ensembles: orthogonal $(\beta=1)$, unitary $(\beta=2)$ or symplectic $(\beta=4)$. The matrix elements of $H$ are uncorrelated random variables with a Gaussian probability distribution with zero mean and variance $\lambda^{2} / \beta M$; the later determines the mean level spacing at the center of the band, $\Delta=\pi \lambda / M$ [37]. An ensemble of Hamiltonian matrices leads to an ensemble of $S$-matrices, which represents the several realizations of systems for which the statistical analysis is performed. To implement the simulations we follow the same method as in Ref. [40] for $\beta=1$ and 2, while for $\beta=4$ the subroutine given in Ref. [41] was used to generate the random Hamiltonian.

For each realization we diagonalize the matrix $S$ to determine its eigenvalues. We are only interested in one of them, $E_{s}(\varepsilon)=\exp \left[\mathrm{i} \theta_{s}(\varepsilon)\right]$ let say, but evaluated at three energies in order to calculate the energy derivative. That is,

$$
\tau_{s}=-\frac{\mathrm{i}}{2 \pi \epsilon} \frac{E_{s}(\epsilon / 2)-E_{s}(-\epsilon / 2)}{E_{s}(0)},
$$

where $\epsilon=\varepsilon / \Delta$.

In Fig. 1 we compare the theoretical distribution of Eq. (14), for $\beta=1$, with the numerical results obtained from the random matrix simulations with $10^{5}$ realizations of $\tau_{s}$, calculated as in Eq. (16) for $M=100$ and $\epsilon=0.001$. We observe a good agreement for the several cases of $N$ presented. This result is an important one since it had not been verified numerically before. Figure 2 shows the corresponding comparison for $\beta=2$, which is in agreement with those of Ref. [33]. For $\beta=4$ the theoretical result fits well with the numerical simulation as can be seen in Fig. 3. Let us note that this is the first time that the distribution of the partial times is verified for $\beta=4$.

\section{Conclusions}

Based on known results of the joint moments of proper delay times, we obtained the distribution of the partial delay times, for an arbitrary number of channels and any symmetry, in the equivalent channels situation. This was done following and inductive method by extracting the underlying part coming from the level repulsion 

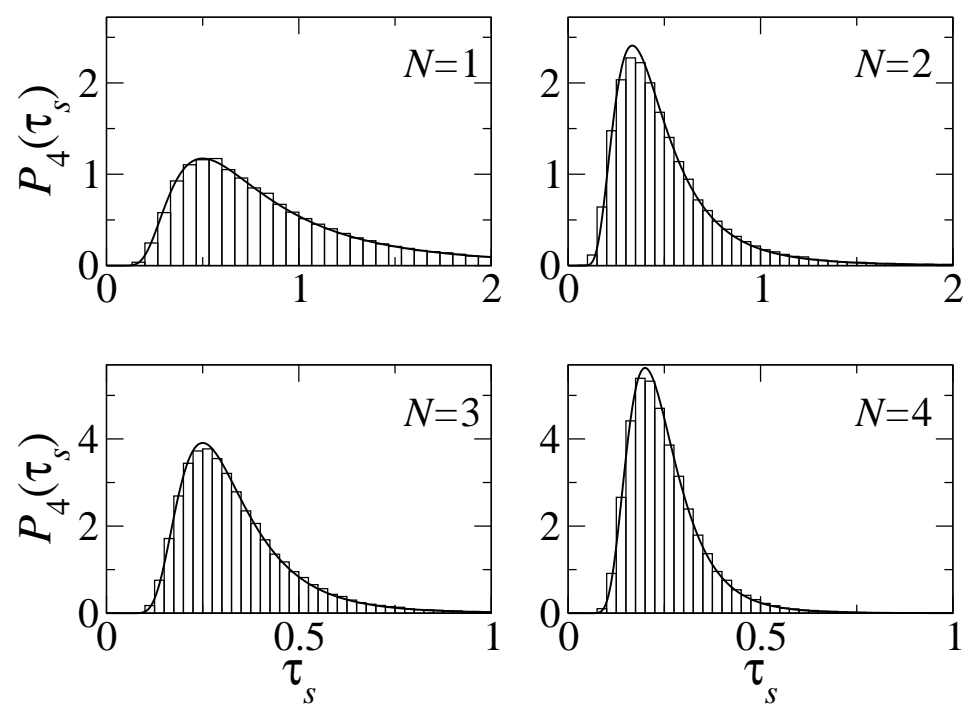

Fig. 3. The same as in Fig. 1 but for $\beta=4$.

that trascends to the $k$ th moment of the proper delay times. This distribution was tested by random matrix theory simulations of ballistic chaotic cavities with ideal coupling, extending its numerical verification to all symmetry classes. This result reproduces the existing expressions for the distribution of partial delay times previously obtained in the literature when properly normalized. Also, we were able to provide the normalization constant for the joint distribution of the proper delay times.

A. M. Martínez-Argüello thanks CONACyT, Mexico, for financial support. A. A. FernándezMarín also thanks financial support from PRODEP under the project No. 12312512. M. Martínez-Mares is grateful with the Sistema Nacional de Investigadores, Mexico.

\section{References}

1. E.P. Wigner, Phys. Rev. 98, (1995) 145

2. F.T. Smith, Phys. Rev. 118, (1960) 349

3. L. Lyuboshits, Phys. Lett. B 72, (1977) 41

4. M. Bauer, P.A. Mello, K.W. McVoy, Z. Phys. A 293, (1979) 151

5. R. Landauer, Th. Martin, Rev. Mod. Phys. 66, (1994) 217

6. P.J. Price, Phys. Rev, B 48, (1993) 17301

7. G. Iannaccone, Phys. Rev. B 51, (1995) 4727

8. G. Berkolaiko, J. Kuipers, J. Phys. A: Math. Theor. 43, (2010) 035101

9. F. Mezzadri, N.J. Simm, J. Math. Phys. 52, (2011) 103511

10. F. Mezzadri, N.J. Simm, J. Math. Phys. 53, (2012) 053504

11. J. Kuipers, D.V. Savin, M. Sieber, New J. Phys. 16, (2014) 123018

12. M. Marciani, P.W. Brouwer, C.W.J. Beenakker, Phys. Rev B 90, (2014) 045403

13. M. Novaes, J. Math. Phys. 56, (2015) 062110

14. M. Novaes, J. Math. Phys. 56, (2015) 062109

15. J. Feist, O. Zatsarinny, S. Nagele, R. Pazourek, J. Burgdörfer, X. Guan, K. Bartschat, B.I. Schneider, Phys. Rev. A 89, (2014) 033417

16. I.A. Ivanov, A.S. Kheifets, Phys. Rev. A 89, (2014) 043405

17. P.C. Deshmukh, A. Mandal, S. Saha, A.S. Kheifets, V.K. Dolmatov, S.T. Manson, Phys. Rev. A 69, (2014) 053424 
18. A. Chacon, M. Lein, C. Ruiz, Phys. Rev. A 89, (2014) 053427

19. M. Schultze, M. Fie $\beta$, N. Karpowicz, J. Gagnon, M. Korbman, M. Hofstetter, S. Neppl, A.L. Cavalieri, Y. Komninos, Th. Mercouris, C.A. Nicolaides, R. Pazourek, S. Nagele, J. Feist, J. Burgdörfer, A.M. Azzeer, R. Ernstorfer, R. Kienberger, U. Kleineberg, E. Goulielmakis, F. Krausz, V.S. Yakovlev, Science 328, (2010) 1658

20. J. Lambe, R.C. Jaklevic, Phys. Rev. Lett. 22, (1969) 1371

21. M. Büttiker, J. Phys.: Condens. Matter 5, (1993) 9361

22. M. Büttiker, A. Prêtre, H. Thomas, Phys. Rev. Lett. 70, (1993) 4114

23. S.A. van Langen, P.G. Silvestrov, C.W.J. Beenakker, Superlatt. Microstruct. 23, (1998) 691

24. P.W. Brouwer, S.A. van Langen, K.M. Frahm, M. Büttiker, C.W.J. Beenakker, Phys. Rev. Lett. 79, (1997) 913

25. P.W. Brouwer, Phys. Rev. B 58, (1998) R10135

26. P.W. Brouwer, K.M. Frahm, C.W.J. Beenakker, Waves Random Media 9, (1999) 91

27. Y.V. Fyodorov, H.-J. Sommers, J. Math. Phys. 38, (1997) 1918

28. P.W. Brouwer, K.M. Frahm, C.W.J. Beenakker, Phys. Rev. Lett. 78, (1997) 4737

29. D.V. Savin, Y.V. Fyodorov, H.-J. Sommers, Phys. Rev. E 63, (2001) 035202(R)

30. V.A. Gopar, P.A. Mello, M. Büttiker, Phys. Rev. Lett. 77, (1996) 3005

31. Y.V. Fyodorov, D.V. Savin, H.-J. Sommers, Phys. Rev. E. 55, (1997) R4857

32. Y.V. Fyodorov, H.-J. Sommers, Phys. Rev. Lett. 76, (1996) 4709

33. P. Šeba, K. Życzkowski, J. Zakrzewski, Phys. Rev. E 54, (1996) 2438

34. A.M. Martínez-Argüello, M. Martínez-Mares, J.C. García, J. Math. Phys. 55, (2014) 081901

35. M.L. Mehta, Random Matrices (Academic, New York 1991)

36. F.J. Dyson, J. Math. Phys. (N.Y.) 3, (1962) 140

37. T. Guhr, A. Müller-Groeling, H. A. Weidenmüller, Phys. Rep. 299, (1998) 189

38. J.J.M. Verbaarschot, H.A. Weidenmüller, M.R. Zirnbauer, Phys. Rep. 129, (1985) 367

39. C.W.J. Beenakker, Rev. Mod. Phys. 69, (1997) 731

40. M. Martínez-Mares, C.H. Lewenkopf, E.R. Mucciolo, Phys. Rev. B 69, (2004) 085301

41. V. Cappellini's home page, http://users.ictp.it/ valerio/HTML/ForTran/RMT.html 